\title{
MESOSCALE SPATIAL AND TEMPORAL VARIATIONS OF \\ WATER MASS CHARACTERISTICS IN THE \\ CALIFORNIA CURRENT REGION OFF \\ MONTEREY BAY IN 1973-1974
}

Richard Edward Blumberg 



\section{NAVAL POSTGRADUATE SGHOOL Monterey, California}
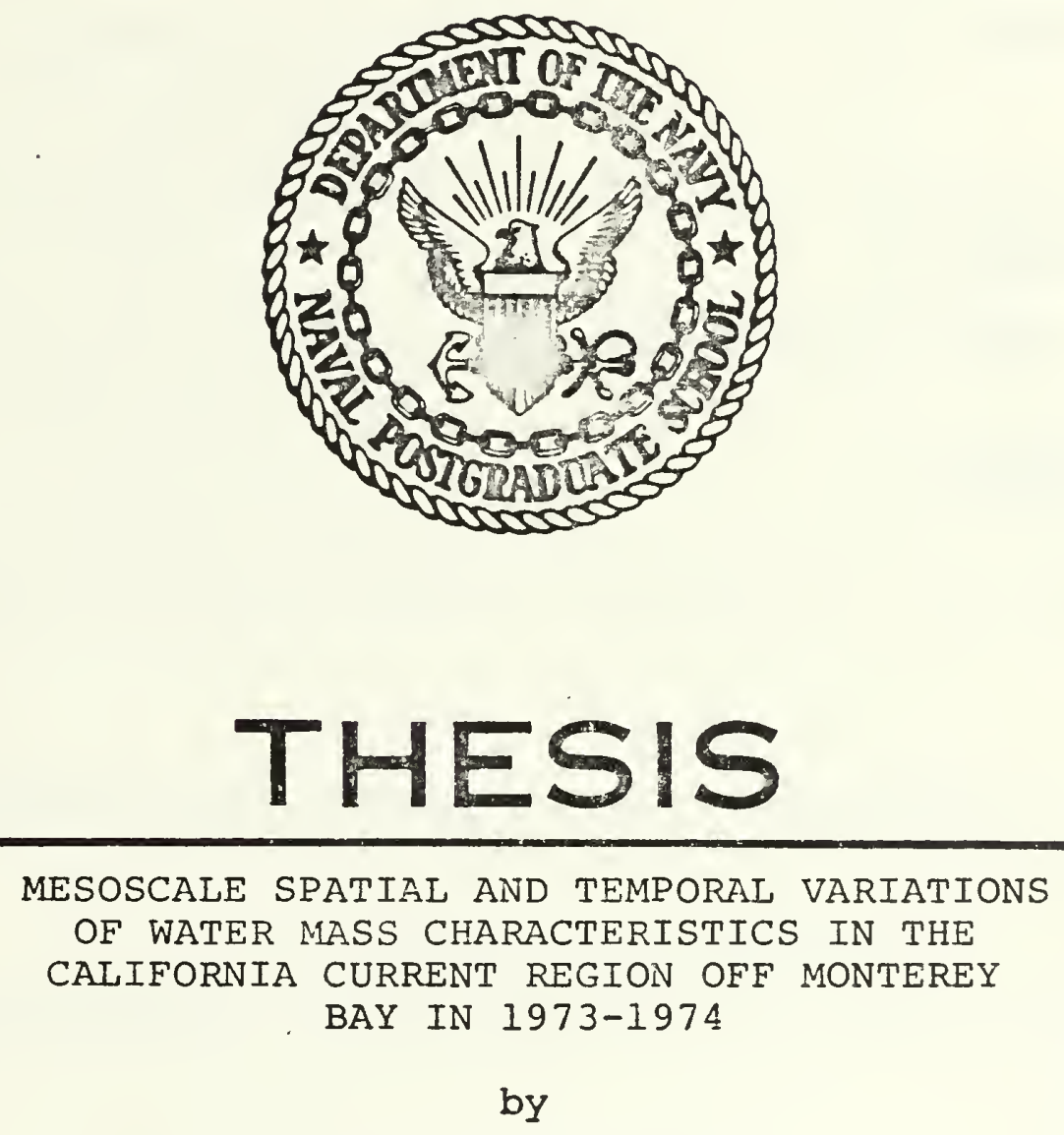

Richard Edward BIumberg

September 1975

Thesis Advisor: Jacob B. Wickham

Approved for public release; distribution unlimited. 



\begin{tabular}{|c|c|}
\hline REPORT DOCUMENTATION PAGE & $\begin{array}{l}\text { READ INSTRUCTINNS } \\
\text { BEFORE COMPLETIAC FORM }\end{array}$ \\
\hline 2. GOVT ACCESSION NO. & 3. RECIPIENT'S CATALOG NUMBER \\
\hline $\begin{array}{l}\text { Mesoscale Spatial and Temporal Variations } \\
\text { of Water Mass Characteristics in the } \\
\text { California Current Region off Monterey } \\
\text { Bay in 1973-1974 }\end{array}$ & $\begin{array}{l}\text { 3. TYPE OF REPORT A PERIOD COVEREO } \\
\text { Master's ThESIS } \\
\text { September } 1975 \\
\text { 6. PERFORMING ORG. REPORT NUMEER }\end{array}$ \\
\hline $\begin{array}{l}\text { 7. AUTHOR(•) } \\
\text { Richard Edward Blumberg }\end{array}$ & 6. CONTRACT OR GRANT NUMBER(•) \\
\hline $\begin{array}{l}\text { 9. PERFORMING ORGANIZATION NAME AND ADDRESS } \\
\text { Naval POStgraduate SchoOl } \\
\text { Monterey, California } 93940\end{array}$ & $\begin{array}{l}\text { 10. PROGRAMELEMENT, PROJECT, TASK } \\
\text { AREA A WORK UNIT NUMBERS }\end{array}$ \\
\hline \multirow{2}{*}{$\begin{array}{l}\text { 11. CONTROLLING OFFICE NAME AND AODRESS } \\
\text { Naval Postgraduate School } \\
\text { Monterey, California } 93940\end{array}$} & $\begin{array}{l}\text { 12. REPORT DATE } \\
\text { September } 1975\end{array}$ \\
\hline & $\begin{array}{l}\text { 13. NUMBER OF PAGES } \\
142\end{array}$ \\
\hline \multirow{2}{*}{$\begin{array}{l}\text { T4. MONITORING AGENCY NAME ADDRESS(ll dilforont trom Controllind Olllco) } \\
\text { Naval Postgraduate School } \\
\text { Monterey, California }\end{array}$} & $\begin{array}{l}\text { 15. SECURITY CLASS. (ol thie roport) } \\
\text { UnClassified }\end{array}$ \\
\hline & $\begin{array}{l}\text { 150. DECLASSIFICATION/DOWNGRADING } \\
\text { SCHEDULE }\end{array}$ \\
\hline
\end{tabular}

Approved for public release; distribution unlimited.

17. DISTRIBUTION STATEMENT (ol the ebotract entered in Block 20, Il dillerent trom Roport)

18. SUPPLEMENTARY NOTES

19. KEY WORDS (Consinue on Peveree alde II neceveary and ldenslty by block number)

20. ABSTRACT (Continue on peveroe olde II neceeeany and ldentlty by block member)

Continuous salinity and/or temperature profiles were made off the coast of Monterey Bay monthly between August 1973 and August 1974 and on a spatial grid finer than is conventionally used. A procedure is described to convert the data from analog to digital form, process these data on an IBM 360 computer, and print out the results by station for each month.

The results show water mass features of small spatial scale detectable only because of the small grid spacing. A gyre or 

filament structure is suggested by the north to south variation between lines of stations. The extent of the area of the survey is insufficient to describe completely the water mass structure on both the eastern and western boundaries; however, the temporal variation in the observed water mass structure is consistent with the three oceanographic seasons described for the california Current system. Elements of relatively cold water and relatively warm water masses are identified in the area of survey. 

Mesoscale Spatial and Temporal Variations of Water Mass Characteristics in the California Current Region off Monterey Bay in 1973-1974

\section{by}

Richard Edward Blumberg

Lieutenant, United States Navy B.S., University of Maryland, 1967

Submitted in partial fulfillment of the requirements for the degree of

MASTER OF SCIENCE IN OCEANOGRAPHY

from the

NAVAL POSTGRADUATE SCHOOL

September 1975 
B583

c. 1 


\section{ABSTRACT}

Continuous salinity and/or temperature profiles were made off the coast of Monterey Bay monthly between August 1973 and August 1974 and on a spatial grid finer than is conventionally used. A procedure is described to convert the data from analog to digital form, process these data on an IBM 360 computer, and print out the results by station for each month.

The results show water mass features of small spatial scale detectable only because of the small grid spacing. A gyre or filament structure is suggested by the north to south variation between lines of stations. The extent of the area of survey is insufficient to describe completely the water mass structure on both the eastern and western boundaries; however, the temporal variation in the observed water mass structure is consistent with the three oceanographic seasons described for the California Current system. Elements of relatively cold water and relatively warm water masses are identified in the area of survey. 

TABLE OF CONTENTS

I. INTRODUCTION- 9

A. CALIFORNIA CURRENT SYSTEM-_. 9

B. PREVIOUS STUDIES--

C. OBJECTIVES-

II. DATA COLLECTION AND PROCESSING------ 15

A. DATA COLLECTION--

B. CALIBRATION- 16

C. DATA PROCESSING-

1. Trace Preparation------------------ 19

2. Calma Digitizer-------------------- 19

III. RESULTS--

IV. RECOMMENDATIONS FOR FURTHER WORK----------- 29

APPENDIX A-- ----10

APPENDIX B-------

APPENDIX C--

APPENDIX D--

APPENDIX E-----

APPENDIX F-- $-----1---13$

APPENDIX G- -

APPENDIX H--

BIBLIOGRAPHY

INITIAL DISTRIBUTION LIST-- 

LIST OF FIGURES

1. AREA OF SURVEY--

2. STATION LOCATIONS---

3. ISOTHERMS AND CONSTANT SIGMA T SURFACES,

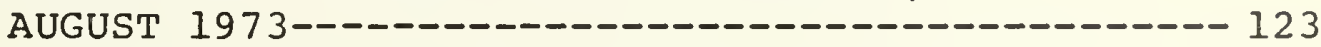

4. ISOTHERMS AND CONSTANT SIGMA T SURFACES, AUGUST 1973-- 194

5. ISOTHERMS AND CONSTANT SIGMA T SURFACES, AUGUST 1973-_ 125

6. ISOTHERMS AND CONSTANT SIGMA T SURFACES, OCTOBER 1973-- 126

7. ISOTHERMS AND CONSTANT SIGMA T SURFACES, NOVEMBER 1973-_- 127

8. ISOTHERMS AND CONSTANT SIGMA T SURFACES,

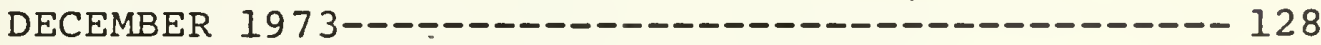

9. ISOTHERMS AND CONSTANT SIGMA T SURFACES, JANUARY $1974-0---129$

10. ISOTHERMS, FEBRUARY 1974--

11. ISOTHERMS, MARCH $1974-(131$

12. ISOTHERMS, MARCH $1974-\ldots$

13. ISOTHERMS, MAY $1974-\ldots$

14. ISOTHERMS, MAY $1974-(34$

15. ISOTHERMS, JUNE $1974-\ldots$

16. ISOTHERMS, JUNE $1974--(36$

17. ISOTHERMS, JULY $1974---137$

18. ISOTHERMS, JULY $1974---\infty---138$

19. ISOTHERMS AND CONSTANT SIGMA T SURFACES, AUGUST $1974---------------------------139$ 



\section{LIST OF TABLES}

I. TYPICAL HEADER LABEL- 31

II. DEPTH, TEMPERATURE AND SALINITY SCALES------ 



\section{ACKNOWLEDGEMENTS}

This thesis involves many to whom I owe much for their encouragement and support. My special thanks go to LCDR Charles E. Workman and Mr. Paul D. Stevens of the Fleet Numerical Weather Central for their assistance in providing the digitizer facilities. I would like to thank Associate Professor Robert G. Paquette, Mannas Anderson, Kristina L. Butler and Edwin V. Donnellan for their advice on running the computer programs and their assistance in their preparation and ltilization. I would also like to thank Richard E. Greer and John G. Hughes for their assistance in working up the data. I would like to express my sincere appreciation to my advisor, Associate Professor Jacob B. Wickham, for his encouragement of this project and his recommendations for its content. 



\section{INTRODUCTION}

\section{A. CALIFORNIA CURRENT SYSTEM}

The California Current system has been described in terms of three general periods based on the particular dominant feature which occurs during that part of the year. The three periods are: the Davidson period (mid-November to midFebruary), the upwelling period (mid-February to the end of July) and the oceanic period (end of July to mid-November). The different conditions that exist during these periods are the result of the offshore currents and the predominant winds [Sverdrup, Johnson, and Fleming, 1942].

Two currents are important. The first, the California Current, is formed by parts of the North Pacific Current and the Subarctic Current and flows southward along the west coast of the United States between $48^{\circ} \mathrm{N}$ and $23^{\circ} \mathrm{N}$. The current is wide and flows sluggishly to the southeast until, off Central America, the current turns west and becomes the North Equatorial current.

The second important current is the relatively warm Davidson Current. This is a coastal countercurrent inshore of the California Current along the west coast of the United States [Glossary of Oceanographic Terms, 1966]. It may be present as a surface or as a subsurface current and has also been called the California Countercurrent by some investigators. 

The California Current system is found between a cell of high atmospheric pressure to the west and a low pressure cell over the land to the east. The western cell often dominates; thus the winds over the California Current are generally from the north and west. These pressure cells change their relative positions and strengths, and cause a seasonal variation in the winds along the coast. For example, between spring and fall the winds are primarily northerly. When strong enough and combined with the Coriolis Force this wind drives the surface water away from the coast giving rise to upwelling. In the winter the northerly component of the wind weakens or reverses. This corresponds to the period when the Davidson Current flows northward on the surface [Edmisten, 1974]. In addition to the variability in the current structure due to the interaction with the local wind, other factors, including changes in global scale wind patterns, contribute to both the long term and the short term changes in the water mass distribution.

\section{B. PREVIOUS STUDIES}

The distribution of the water masses and the interaction of the current off the west coast of California have been examined by a number of investigators whose works are summarized by Molnar, Brown, and Edmisten. Only some of the more interesting characteristics of water mass distribution within the California Current system will be described here. 

The nature of the Davidson Current changes with the season. During the Davidson period the countercurrent is present at all depths on the coastal side of the California Current to at least $48^{\circ} \mathrm{N}$ latitude. The upper homogeneous layer is relatively thick, that is, the temperature at $25 \mathrm{~m}$ is nearly the same as the surface temperature and at $50 \mathrm{~m}$ the temperature is only slightly lower [Sverdrup, et al., 1942]. Satellite reconnaissance of the California coastal currents between July 1972 and May 1974 provides an additional source of information on the surface current patterns. The ERTS-1 (Earth Resources Technology Satellite) imagery analysis of the Monterey Bay area showed a very uniform surface current pattern during the Davidson Current period. The horizontal variation was very slight although no regular pattern of water mass distribution was discernible. The northward flowing Davidson Current was not observed to be present within about $30 \mathrm{~km}$ offshore during November and December 1973. However, it was recorded to be present on the surface in January and February 1973 [Pirie, and Steller, 1974].

As the winds shift to the north, or northwest in early spring the Davidson Current breaks up into a series of eddies as upwelling intensifies [Brown, 1974]. The Davidson Current is still present below 200 to $250 \mathrm{~m}$, and according to Sverdrup, in the absence of the winds which cause the upwelling, the Davidson Current would be present on the surface. The upwelling period is characterized by a rise in the 

isotherms toward the coast where the temperature is about $3^{\circ} \mathrm{C}$ lower than further offshore. The water which is carried toward the surface is from the upper $200 \mathrm{~m}$. Below $200 \mathrm{~m}$ the slope of the isotherms is opposite to those in the surface layer. This downward slope may be indicative of a reversal of current direction toward the north [Neumann and Pierson, 1966].

During the Oceanic period the California Current is dominant throughout the area of survey. However, the Davidson Current is still present at depth.

The ERTS-1 imagery analysis illustrated the complexity and variability in the current structure. Several months showed both warm and cold components to be present offshore (January and February 1973), while other months indicated no definite flow direction but showed a series of gyres and area of mixture of the water of the two currents (April, September, October, November and December 1973) [Pirie, et al., 1974]. This complex structure is in agreement with the work of Scripps Institution of Oceanography as reported by Sverdrup. In the areas of intense upwelling tongues of water of low temperature extend southward away from the coast and between these tongues are higher temperature tongues extending in toward the coast. The flow within the warm tongues is directed toward the north and the flow within the cold tongues is directed toward the south. 

Reid, Roden and Wyllie [Brown, 1974] described four water masses that contribute to the California Current system. The Subarctic Water Mass and the Central Water Mass come in from the west and northwest. The Davidson Current is from the Equatorial Water Mass to the south. The fourth water mass is the result of the upwelling process along the coast. This classification scheme was later simplified to a "northern" water mass called Subarctic North Pacific Water and a "southern" water mass called Equatorial Pacific Water [Brown, 1974].

The terms "northern" and "southern" water will be used in this paper to describe the water mass characteristics in the area of study, rather than their places of origin. Northern water is water which appears colder and less saline than the water of the same density surrounding it. Similarly, southern water is water which is relatively warmer and saltier than the surrounding water of the same density.

\section{OBJECTIVES}

The primary goal of this study is to describe the annual variation in the structure of the water mass distribution in the area of survey and to document the procedure which was used to process the data. This description is based on a year's monthly observations using continuous profiling sensors. Specifically, the following points are discussed:

1. A procedure for processing a large quantity of analog salinity, temperature and depth (STD) data and expendable bathythermograph (XBT) data is described. 

2. Two computer programs to process this data were modified and are documented in Appendices $D$ and $E$.

3. The water mass distribution is examined on a small spatial scale (5 $\mathrm{km}$ between stations) in order to see on what scales the variation occurs.

4. The extent of the survey area is considered and its sufficiency to describe the water mass distribution is discussed.

5. The water mass properties were observed once a month for 13 months in order to describe the annual variation in the water masses. 



\section{DATA COLLECTION AND PROCESSING}

\section{A. DATA COLLECTION}

The oceanographic research vessel, Acania, was used to collect data monthly from August 1973 to August 1974 under the supervision of J. B. Wickham of the Naval Postgraduate School. The instrumentation, navigation, and data collection procedures are detailed in Reference 10. Briefly, a BissetBerman continuous profiling salinity, temperature, depth recorder (STD) was used to collect data during the months August 1973 through January 1974, and August 1974. From February through July 1974, the STD was inoperative and data were collected using expendable bathythemographs (XBT). No data were collected in April 1974 and insufficient data were collected in September 1973 due to inclement weather. Independent measurements of salinity and temperature were made at selected depths using Nansen bottles, a conductivity salinometer, and reversing thermometers during both the STD and the XBT cruises.

Figure 1 shows the area which was examined and Figure 2 shows the location of the stations, although not all of the stations were sampled each month. As noted in Reference 10, the use of a station spacing of $5 \mathrm{~km}$ is a finer grid than is traditionally used in oceanographic survey work but it is necessary to define narrow streams of northern and southern water. 



\section{B. CALIBRATION}

The vertical definition as a result of collecting the data with the Bisset-Berman continuous profiling STD is estimated to be a few meters or less. This is based on a rate of drop of about 30 meters per minute.

After the data had been collected the STD and XBT traces were compared with the reversing thermometer temperatures or the salinities from the Nansen bottles to determine if a temperature correction (TCOR) or a salinity correction (SCOR) was necessary. TCOR was determined by plotting the value of temperature from the trace, $\mathrm{T}_{\mathrm{STD}}$ or $\mathrm{T}_{\mathrm{XBT}}$ ' along one axis against the temperature from the reversing thermometers, $T_{N}$, along the other axis for the same depth. The number of points plotted was a function of the number of Nansen casts taken at all stations for the particular month in question. If $\mathrm{T}_{\mathrm{STD}}$ and $\mathrm{T}_{\mathrm{N}}$ were the same for each point, a $45^{\circ}$ line through the origin would connect the points. If the best fit line did not pass through the origin the constant correction, TCOR, was applied to shift the line back to the origin.

$$
\mathrm{TCOR}=\mathrm{T}_{\mathrm{N}}-\mathrm{T}_{\mathrm{STD}}
$$

The salinity correction, SCOR, was determined in a similar manner using the salinity of the water in the Nansen bottles and the salinity on the STD traces.

$$
\mathrm{SCOR}=\mathrm{S}_{\mathrm{N}}-\mathrm{S}_{\mathrm{STD}}
$$



The XBT traces were standardized to reversing thermometer temperatures in a similar manner except that TCOR was determined from the $Y$ intercept of a straight line through the various points computed by the WLQP 2 subroutine in the $W . R$. Church Computer Center subroutine library. WLQP2 is a least square polynomial fit and, by calling for the best fit first order polynomial, a straight line through the data points is produced. The data points were again determined by using the temperature from the XBT trace and the temperature from the reversing thermometer of the Nansen casts at the same depth.

During several months there were no Nansen casts taken at the XBT stations. However, there were a number of stations at which both STD and XBT data were collected, and still other stations at which Nansen and STD data were collected. This enabled a determination of the temperature correction, TCOR, for the STD data as explained above:

$$
\text { TCOR }=T_{N}-T_{S T D}
$$

The XBT data were then compared to the corrected STD data and a constant correction, XBTCOR, was determined and applied to the XBT data for that particular month.

$$
\mathrm{XBTCOR}=\mathrm{T}_{\mathrm{STD}}-\mathrm{T}_{\mathrm{XBT}}
$$

Generally, the XBT temperatures were higher than the STD temperatures and, by applying the XBTCOR correction, a reasonable comparison between STD and XBT data can now be made. 

One final check on the corrected data was conducted after the processing computer programs, DIGISTD and DIGIXBT, had been run. The computer printouts for a number of stations were examined at randomly selected depths. These values of temperature or salinity were compared with the values read from the traces. The computer printouts and the traces were in agreement after the constant correction, TCOR, SCOR or XBTCOR, had been applied.

\section{DATA PROCESSING}

To prepare the data for analysis the STD and XBT traces were examined visually, smoothed by removing spurious spikes, and marked for ease of digitizing. They were then taken to Fleet Numerical Weather Central, Monterey, where the data were converted from analog to digital form using a Calma company digitizer. Operation of the Calma digitizer is explained in Appendices $\mathrm{A}$ and $\mathrm{B}$. The digitizer records the data on a 556 bpi, 7-track magnetic tape which was then processed at the $W$. R. Church Computer Center using the appropriate program, DIGISTD or DIGIXBT. The computer programs are listed and explained in Appendices $D$ and $E$. The resulting output is a listing by station of depth and temperature for the XBT data. The STD output consists of a listing by station of depth, temperature, salinity and the calculated value of sigma $t$. Sound velocity is also calculated for the STD traces but will not be discussed in this paper. The computer output can be 

in one of several forms: a paper printout, a punched card printout or a 9-track magnetic tape. The 9-track tape was used to supply the data for the plotting programs XBTPLT or STDPLT which are listed in Appendices $F$ and $G$.

\section{Trace Preparation}

The individual STD or XBT traces were treated in the same general manner. The STD salinity trace required special preparation since spikes are artificially introduced in the salinity trace by the equipment [Wickham, 1975]. The up and down traces were stapled together so the traces were superimposed. If a spike appeared on one trace and not on the other or if two spikes were oriented oppositely, they were ignored. The down trace was then outlined in pencil to make it easier for the digitizer operator to record the data. The XBT and STD temperature traces were traced only in areas where the original ink was difficult to see because it was smeared or too light to be digitized easily. A small circle was drawn to mark the beginning and end of each segment of the trace. A segment is an entire section of the trace recorded at a single set of depth, temperature and salinity scales. If one scale changes, the pen drawing of the STD trace is repositioned and a new segment is started.

\section{Calma Digitizer}

The Calma Company Model 480 digitizer reduces analog graphical data to digital form for computer processing and analysis. Two machines were used to process the data and 

are located at the Fleet Numerical Weather Central, Monterey, California.

Reference 2 describes the operation of the digitizer. To digitize analog graphical data directly onto computercompatible magnetic tape, the operator manually traces the graphical data with a movable stylus/carriage assembly. The location of the stylus is transmitted through cables to magnetic reluctance encoders, which convert stylus movement to digital signals. The signals are processed and formated for output on a 556 bpi, 500 cps, magnetic tape. Also recorded on the tape, through a manual keyboard entry, are the required identification codes such as station number, month, scaling factors, inter-record gap, error correction and others as desired, which are used by the final processing computer programs.

The maximum sampling interval for the STD data was 0.02 inch of stylus movement in either the $\mathrm{X}$ or $\mathrm{Y}$ direction. This sampling interval is a function of the stylus speed and, for most of the data, the stylus position was recorded at every .01 inch. The .02 inch sampling interval corresponds to a maximum depth change between recorded data points of approximately $.66 \mathrm{~m}$ for the 0 to $300 \mathrm{~m}$ scale, and $1.75 \mathrm{~m}$ for the 0 to $750 \mathrm{~m}$ scale. The maximum temperature change between data points is about $.01^{\circ} \mathrm{C}$, and the maximum salinity change between data points is about .004 ppt. 

For the XBT data the .02 inch corresponds to a maximum depth change between recorded data points of $2.0 \mathrm{~m}$ and a temperature change of $.1^{\circ} \mathrm{C}$. The Calma digitizer itself has a maximum absolute error of .012 inches or $0.1 \%$. In summary, the error introduced during the digitizing procedure is more significant for the XBT data than the STD data but is still very slight over the range of depth and temperature. 



\section{RESULTS}

Figures 3 through 19 are vertical cross sections of the area of survey showing the isotherms between $6.5^{\circ} \mathrm{C}$ and $12.0^{\circ} \mathrm{C}$ for each month. Figures 3 through 9, and 19 are based on the STD data and have lines of constant sigma $t$ (isopycynals) plotted in addition to the isotherms. The XBT data are the basis for Figures 10 through 18 and, since there were not enough Nansen stations to determine the salinity distribution, the density structure is not shown.

The figures were plotted by using the CONTUR subroutine from the W. R. Church Computer Center subroutine library. This program does not allow for gaps in the data. Therefore, the depth of the plots is limited to the depth of the shallowest station taken during each month. In certain cases a particular station was either too shallow or the data were too unrealistic to be plotted, or there were no data taken for that particular station at all. The net result is that the figures must be examined carefully because the station spacing is not uniform. The stations appear uniformly spaced even if the actual distance between stations is not constant.

The shaded areas on the figures represent the major areas of northern and southern water. For example, in Figure 3 the warm southern water is characterized by isotherms which dip below the 26.7 sigma t surface at stations 110 and 111 . This slope of the isotherms is much steeper than the depression in the 26.9 sigma $t$ surface just below and indicates the 

presence of water which is warmer than the adjacent water of the same density and depth at station 109.

The cold northern water has isotherms which slope upward more steeply or rise up above the constant sigma $t$ surface. This is particularly evident in Figure 3 at station 108 on the 26.3 sigma $t$ surface. At the other density surfaces for station 108 the isotherms and density surfaces both slope upward in the same general direction but the slope of the isotherms is more pronounced. The difference in the slopes of the isotherms and the isopycnals is important because it differentiates between local vertical motion due to internal waves or upwelling and differences in water mass structure due to horizontal advection.

An exception to this which is particularly important in analyzing the XBT data is the apparent slope in the isotherms which is due to bad data. For example, station 303 in Figure 12 has each isotherm depressed through the entire water column giving the appearance of being a relatively warm water intrusion. Because the depression is limited to the one station and appears over the entire $450 \mathrm{~m}$ depth, it is probably due to a faulty XBT, a misaligned recording trace, or improper digitizing.

Another problem which is unique to the XBT is illustrated in Figure 17 between stations 202 and 204. The surface values down to a depth of about $300 \mathrm{~m}$ appears to be normal in that the isotherms are horizontally consistent. Below $300 \mathrm{~m}$ an 

apparent narrow warm intrusion appears at station 204. This anomaly is probably instrumental since the adjacent station 203 is consistent with station 205. The problem is that the XBT has a limited quantity of wire available. The recommended depth for this group of sensors was $450 \mathrm{~m}$. If the wire is tangled or if the probe goes deeper than the wire is long, the wire will be stretched for a period of time before it breaks. When this wire is stretched, the recorded temperature becomes erratic. It is difficult to differentiate a real change in temperature from this artificial anomaly. In order to validate this sort of temperature change at depth, some sort of independent measurement is required, perhaps with another XBT or with a Nansen bottle and reversing thermometers. Although a number of Nansen casts were made, there were none taken at these stations. Because there are no isopycnals nor enough independent measurements to verify the structure indicated by the isotherms, it is not possible to state positively whether a specific feature is real or artificial. The following figures contain structures which appear to be the result of bad or stretched XBT's: Figure 12 - station 303, Figure 14 - station 315, Figure 17 - stations 204 and 202, and Figure 18 - station 312 .

The figures will be discussed now in terms of the three oceanographic seasons. Figures 3, 4, and 5 are from August 1973. This is during the oceanic period which is from July through November. They show the presence of the southern 
$$
\text { . }
$$ 
water below $200 \mathrm{~m}$ in the southern section of the area of survey. The warm water is present about $45 \mathrm{~km}$ offshore in Figure 3. Figure 4 is from the stations $20 \mathrm{~km}$ to the north where the warm water is present about $30 \mathrm{~km}$ off the coast. However, the warm water is scarcely indicated at all in Figure 5 which is $20 \mathrm{~km}$ further north. This shows the diversity in the nature of the water mass distribution. It is not known if the warm water is present further to the west, deeper than $500 \mathrm{~m}$ (which is unlikely) or just missing. This would indicate that the warm water to the south is merely part of a filament or gyre that only reached so far north or is discontinuous.

Figures 6 and 7, also from the oceanic period, show the presence of the warm southern water on the western boundary of the area of survey. It is still $100 \mathrm{~m}$ below the surface. In both cases a large cold water mass is adjacent to, and on the coastal side of, the warm water.

The Davidson period is between November and February. Figures 7, 8, 9 and 10 correspond to this period and show the presence of southern water at depth. The Davidson Current was expected to be observed on the surface at least in January and February as reported for the previous year in Reference 8. Figure 9 does show a warm water area near the surface in January 1974.

The upwelling period from February to July corresponds to Figures 10 through 18. The upwelling is expected to be 

confined to the upper $200 \mathrm{~m}$, and may be a very localized feature. The isotherms are expected to slope upward on the coastal side of the graphs. February (Fig. 10), May (Figs. 13 and 14), and possibly June (Fig. 15) do show upwelling at the eastern boundary. However, it is not clear from the graphs what the nature of the structures are since there are no data further east.

Another prominent feature exhibited on the graphs from May to July is a large warm water mass below $200 \mathrm{~m}$ on the eastern edge of the area of survey. Some downward sloping of the isotherms is expected below the areas of upwelling in the presence of a countercurrent as suggested in Reference 7. This theory is further supported by the existence of the same sort of large warm water structure which is seen in Figure 19 for August 1974. The data in Figure 19 are supported by the density structure in that the isotherms and isopycnals do diverge and this indicates the presence of relatively warmer and saltier water.

A comparison of the structure between August 1973 and August 1974 is of interest. August 1973 (Figures 3, 4 and 5) was discussed earlier. The warm water masses are distributed in relatively small areas to the west and central portions of the survey area and very little warm water is present in Figure 5 for the northern stations. The cold northern water is present in August 1973 and is very strong for the 100 and 200 series stations. August 1974 exhibits 

structure which is sharply different in that a large mass of warm water is present on the eastern boundary of the survey area and no cold water is obvious. The warm water is present at depth, which is consistent with the previous year and is as expected during the oceanic period. The difference between the months illustrates that although there may be a long time average annual cycle, the short term deviations are very large.

In summary, the data processing procedures to handle a large quantity of analog temperature and salinity data were developed and are detailed in the appendices. This study shows one year's variation in the water mass distribution off the California coast. The nature of these variations dictates the study be made on a small spatial scale. Many of the critical features are less than $10 \mathrm{~km}$ wide. The north to south variation is significant as is shown by the data from August 1973. Southern water structures present along the southern line of stations are not present along the same line just $40 \mathrm{~km}$ to the north. This suggests that the warm and cold water masses are part of a gyre or filament system as opposed to a strong central cored current.

The area of survey does not appear to be extensive enough to record all of the significant features. Upwelling is indicated on the eastern boundary of several figures but the exact nature of this feature is undetermined. Similarly, many features are identified on the western boundary but the 

westward extent is now known and is certainly of interest. Finally, the difference in water mass structure between August 1973 and August 1974 suggests that the secular variation is significant. This is to be expected since the surface current characteristics, direction and speed depend on the prevailing meteorological conditions, which also have a large secular variation. 



\section{RECOMMENDATIONS FOR FURTHER WORK}

The data show areas which support the concept of three basic oceanographic seasons. However, the boundaries do indicate that areas of interest may lie outside of the area of survey. It is recommended that on future cruises data be collected over a wider area, both to the east and the west.

Between February and July 1974, the STD was inoperative. On future cruises if the STD is not operating, it is recommended that a greater number of Nansen casts be made. This would provide independent information on the density structure and also the reversing thermometers would serve to verify the temperatures recorded by the XBTs.

It is also recommended that an initial on board data analysis be made. This makes possible validation of questionable XBT data by means of an immediate resurvey.

One area of study which was not considered here is the short term temporal variation in the water mass distribution. The data from the monthly cruises may not be representative of the predominant conditions that exist in any one month, but may be the result of recent local weather or storm conditions. Although such a survey may be expensive in both time and money, it would provide valuable information on the structure of the water mass variability. 



\section{APPENDIX A}

\section{DIGITIZING PROCEDURE}

The procedure for using the Calma digitizer will be described for an STD trace but is applicable to an XBT trace as well. The only difference is that the XBT trace is complete in one segment, and the STD trace usually requires multiple segments, which correspond to scale changes of depth (D), temperature (T) or salinity (S). Typically, an STD trace consists of three or four temperature segments and two salinity segments. The term record is used to describe a single complete entry on the digitizer tape. This record may be either a header label, which identifies the station and scale factors for the data which follow, or a trace segment which is the actual digitized data for a segment of $\mathrm{T}$ verses $\mathrm{D}$, or $S$ verses D. Appendix B is a checkoff sheet which was used when digitizing the data.

Once the machine is turned on and the magnetic tape is loaded, the first STD trace is taped to the table surface so it will not slip. It is aligned so that the $\mathrm{X}$ axis of the digitizer is parallel to the depth axis of the trace and the $Y$ axis is parallel to the temperature/salinity axis. It is important that the trace be rotated $90^{\circ}$ and aligned in this manner because DIGISTD and DIGIXBT expect to process data obtained from a trace with this orientation. (The orientation is used because it is easier to control the digitizer stylus 

moving left to right for a continuous change in depth than for movement from top to bottom.)

The digitizer has two modes of operation, keyboard and tracer modes. The keyboard mode is used for entering the header label (identification) information on the tape for the data which is digitized and put on the tape when the machine is in the tracer mode. The first thing entered on the tape is the header label for the first station. The elements of the header are named for the acronyns used in the DIGISTD program.

Each of the eleven elements of the header is punched and entered on the tape. No blank spaces are allowed. A typical header is shown in Table $I$. The keyboard symbol and the inter-record gap (IRG) are put on the tape by pushing these keys on the keyboard. The keyboard symbol marks the beginning of the header and the IRG marks the end of the record.

\section{-301k9910430IRG}

\begin{tabular}{|c|c|c|}
\hline SYMBOL & NAME & EXPLANATION \\
\hline$r$ & - & keyboard symbol \\
\hline 301 & ISTAA & station number \\
\hline $\mathrm{K}$ & AMONT & month designation code \\
\hline 99 & IDENT & identifies record as a header \\
\hline 1 & IDSCL & depth scale 1 \\
\hline 0 & ICODE & temperature trace \\
\hline 4 & ITSCL & temperature scale 4 \\
\hline 3 & ISCL & salinity scale 3 \\
\hline 0 & IP & do not print this station yet \\
\hline IRG & IRG & inter-record gap \\
\hline
\end{tabular}

TABLE I. Typical Header Label. This is the first temperature trace segment of an STD trace. 

The first three elements of the header comprise a three digit number, called ISTAA in the computer programs. These elements identify the station at which the data were taken. The fourth element of the header is a one letter code called AMONT. This code identifies the particular month and year of the data. The fifth and sixth elements of the header are 99. This is an identification code, called IDENT, used to designate the header label as a header, if the keyboard symbol is missing. If the fifth and sixth characters are not 99 and there is no keyboard symbol, the record is treated as a trace. The next element of the header is called IDSCL in the programs and represents the depth scale at which the data were recorded. DIGISTD allows for three depth scales, a "l" is for a scale from zero to three hundred meters, a "2" is for a scale from zero to seven hundred and fifty meters, and a "3" is for zero to fifteen hundred meters. DIGIXBT has only one scale, a "I". The eighth element of the header is either a "0" or a "1". This element, ICODE, identifies the trace that follows as either a temperature or a salinity trace. In DIGIXBT ICODE is always "0". The ninth and tenth elements of the header are scaling factors for the temperature and salinity scales of the STD which were used when the data were recorded. Table II shows the temperature and salinity scales that are available and are allowed by the DIGISTD program. Only four temperature scales, 2, 3, 4 or 5, and two salinity scales, 3 or 4 , were used to record the data. ITSCL and ISCL are both "I" for 



\section{DEPTH SCALES}

\begin{tabular}{cl} 
IDSCL $=$ & DEPTH RANGE \\
\hline 1 & 0 to $300 \mathrm{~m}$ \\
2 & 0 to $750 \mathrm{~m}$ \\
3 & 0 to $1500 \mathrm{~m}$
\end{tabular}

\section{TEMPERATURE SCALES}

\begin{tabular}{cc} 
ITSCL $=$ & TEMP RANGE \\
\hline 1 & -2 to $3^{\circ} \mathrm{C}$ \\
2 & 2 to $7^{\circ} \mathrm{C}$ \\
3 & 6 to $11^{\circ} \mathrm{C}$ \\
4 & 10 to $15^{\circ} \mathrm{C}$ \\
5 & 14 to $19^{\circ} \mathrm{C}$ \\
6 & 18 to $23^{\circ} \mathrm{C}$ \\
7 & 22 to $27^{\circ} \mathrm{C}$
\end{tabular}

SALINITY SCALES

ISCL

1

2

3

4

5

6

7
SALINITY RANGE

30.0 to $32.0 \mathrm{ppt}$

31.5 to $33.5 \mathrm{ppt}$

33.0 to $35.0 \mathrm{ppt}$

34.5 to $36.5 \mathrm{ppt}$

36.0 to $38.0 \mathrm{ppt}$

37.5 to $39.5 \mathrm{ppt}$

30.0 to $40.0 \mathrm{ppt}$

TABLE II. Depth, Temperature, and Salinity Scales. 

the DIGIXBT program since there were no temperature scale changes for the XBT trace and no salinity scale at all.

The last element of the header directs the printing of the data. The data are desired only after all segments of a trace have been processed. The stored data are printed out if IP is "I" but not if IP is "O". IP is set equal to "I" only on the last salinity header for a particular STD station. Again, since the XBT trace is a single segment trace, the variable IP is always "I".

After the header label is complete and the IRG key is pushed, the stylus is moved to the origin of the trace. The origin is defined as the intersection of the depth axis with a line perpendicular to it, drawn from the beginning of the trace. The beginning was marked when the data was collected and corresponds to the sensor being at the sea surface. The origin should be marked before the digitizing process starts so it can be located easily. This origin location is necessary because the pen is not always started at the zero meter mark on the record paper. Also, the pens for salinity and temperature are offset from each other about one-eight of an inch to avoid interference between the pens.

When the stylus is on the origin, the tracer mode is activated. The digitizer is then recording the movement of the stylus as it is moved from the origin to the beginning of the trace segment. The beginning of the trace segment is flagged by pressing the " $B$ " and the " $A$ " keys, and then entering this 

"BA" on the tape. The stylus is then traced down the segment to its end where an IRG is put on the tape. The digitizer is placed in keyboard mode by pushing "skip" (activate) and then "keyboard". The "skip" button either activates or deactivates the trace accumulator. With skip activated, the stylus may be moved while in tracer mode without changing the digital record.

Digitizing errors may be corrected as follows: The "delete record" key is used when an error has been made when making either a keyboard or a tracer record. The programs process a delete record by ignoring all the data on the tape between the delete record entry and the last previous IRG. The programs begin processing the data put on the tape after the delete record. In this way a minimum of work has to be redone in the event of an error. Because an error in the heading can be overridden by data cards in the processing programs, numerical errors in the heading code should not be corrected by the delete record procedure. Heading errors are detected by using the TAPEOUT program that is explained in Appendix C. The TAPEOUT printout is compared with the desired header to ensure that the codes are correct.

This process of header label followed by trace is continued until all the segments of a trace are recorded. At the end of the tape the "EOF" (end of file) button located on the tape recorder is pushed three times to mark the end of the data on that tape. The tape is rewound and taken to the 

W. R. Church Computer Center for processing by way of the DIGISTD or DIGIXBT programs. 



\section{APPENDIX B \\ DIGITIZER OPERATOR PROCEDURES CHECK-OFF SHEET}

The following list is a check-off sheet which is a useful guide when using the digitizer.

1. Initịal conditions
A. Transformer on
B. $X-Y$ plotter off
c. Tape recorder is left on at all times since power is supplied from the transformer.

2. Load tape

A. Ensure write permit ring is inserted in tape reel.

B. Load tape and run forward to the load point by pushing load forward button.

3. Turn the $X-Y$ plotter on (aural tone is heard).

A. Press record error (aural tone deactivated).

B. Ensure skip button is lit (activated) and press keyboard.

4. Mount STD or XBT Trace.

A. Align grid of trace to $X-Y$ plotter by locking $X$ and aligning $Y$ with axis.

B. Ensure depth increasing in $\mathrm{x}$ direction and temp/salinity increasing in $\mathrm{Y}$ direction.

C. Secure trace with masking tape. 

5. Enter eleven keyboard entries. (Listing of header labels should have been prepared before digitizing started.)

6. Press IRG.

7. Ensure stylus on previous marked origin.

8. Press trace, deactivate skip.

9. Move stylus from origin to beginning of trace segment.

10. Flag trace by pressing "B" "A" and "enter".

11. Trace segment with stylus.

12. At end of trace, press IRG, press skip and press keyboard.

13. Repeat steps 5 through 12 for all trace segments on this trace and then go on to the next trace.

14. At the end of the day

A. Ensure skip and keyboard were pressed.

B. Press EOF (end of file) gap button on the tape recorder three times.

c. Move the stylus into the lower right hand corner of the table.

D. Rewind the tape to the load point.

E. Turn off the $X-Y$ plotter.

F. Hand wind tape past load point to dismount tape reel.

G. Turn off the transformer.

Caution: Sudden or very rapid movement of the stylus may cause the cables to come off their pulleys resulting in calibration errors. 
APPENDIX C

\section{TAPEOUT}

A technical note [Raney, 1973] describes the procedure for using the IBM System / 360 model 67 computer to process 7-track tapes and describes several programs which are useful in processing the tape.

The first program that was run on the 7-track tape was a TAPEOUT program.' TAPEOUT is a general purpose tape dump program which is described in detail in Reference 6. Tapeout provides the user with a printout of the recording density $(200,556$, or $800 \mathrm{bpi})$, the tape parity (even or odd) and physical record length (fixed length, fixed blocked, or variable length) and also a partial dump ( 80 characters) of the record. This information is necessary to prepare the job control language (JCL) cards for the DIGISTD and DIGIXBT programs.

The TAPEOUT program listed below is a modification of the program described in Reference 6.

$/ /($ STANDARD JOB CARD $)$

//EXEC TAPEOUT, PARM $=11,1,1,32760^{\prime}$

$/ /$ FTO6001 DD SPACE $=($ CYL,$(6,1))$, SYSOUT $=0$

$/ /$ TAPEIN DD UNIT=2400-1, LABEL $=(, \mathrm{BLP}), \mathrm{VOL}=\mathrm{SER}=\mathrm{UCM} 011$ $/ *$

This modification prints out the complete tape instead of just 80 characters and also provides the necessary density, parity, and record length parameters. By obtaining a printout of the entire tape, the errors which occurred in the 

header during the digitizing procedure are easily identified and located. This is particularly useful during the preparation of the data cards for the DIGISTD, and DIGIXBT programs. For an explanation of the arguments see Reference 6. 

2

$\sum_{1}^{2}$

ヘト

판

기

a

$x$

$\varpi$

0

$z$

ш

a.

a.

$\varangle$

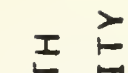

a

แ⿺辶

$0 \propto$

뜽

-U山O>Z

둥의

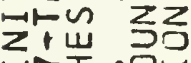

NI・O円

\lrcorner Uwn⿺

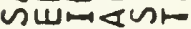

$N$ run

O円न

แเ

NHA

10

-口八ஈU⿺辶

$0 \propto 1 \quad \alpha \alpha$

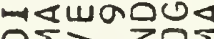

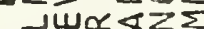

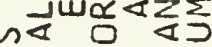

wטo $\rightarrow-4 n$

is wotu

$\sim<\propto \propto ⿻ 0<$

w $0<z \vdash$

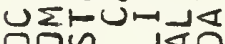

QQn $] \leq 0$

ดแ เயทว

느는

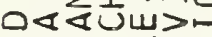

ZL Z $\propto$ r

-

n $1-4$ an

$\vdash I \supset \propto \propto ⿻$

드யUI

யa ₹トดU

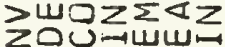

at

vowa ax - Za IOL जदरण

in 0 or

1 षய山 шा

a யxम上0।

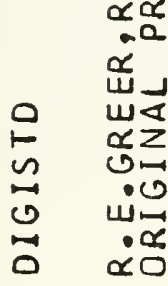

$u+\frac{\alpha}{u}$

山ل

$z$ 这

xw •Ouwnt

जूس $\geq$

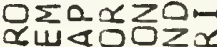

arトu $\cup<a$

\begin{tabular}{|c|c|c|c|c|c|c|}
\hline & & $\propto$ & & & $\leftarrow$ & 0 \\
\hline ш & & $\underline{\underline{ }}$ & & & ш & \\
\hline y & & $I$ & 0 & & $\sim$ & 7 \\
\hline 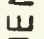 & & 0 & $\alpha$ & & w & 2 \\
\hline 2 & & & 0 & & -1 & $\propto$ \\
\hline$\varnothing$ & $\omega$ & 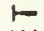 & $\cup$ & & $\infty$ & $\bar{\alpha}$ \\
\hline ш & \lrcorner & $\omega$ & ш & & $<$ & 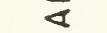 \\
\hline$n$ & $\varangle$ & $n$ & $\propto$ & $\underline{\sim}$ & 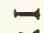 & 1 \\
\hline 0 & 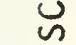 & ש & $\alpha$ & 0 & $\stackrel{\alpha}{1}$ & \\
\hline$z$ & & 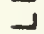 & 0 & 0 & $>$ & $u$ \\
\hline 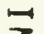 & س & $\infty$ & U & $z$ & & 0 \\
\hline 3 & $\simeq$ & $\varangle$ & & ш & $a$ & \\
\hline & 5 & $=$ & - & & $x$ & $\sim$ \\
\hline 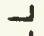 & 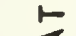 & $\propto$ & $\vdash$ & $\Psi$ & $n$ & 10 \\
\hline 1 & $\varangle$ & $\alpha$ & 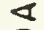 & $I$ & U & $>\alpha$ \\
\hline 0 & $\bullet \propto$ & $>$ & 0 & $\vdash$ & $\propto$ & $\leq 0$ \\
\hline$u$ & जu & $a$ & 0 & $F$ & $z$ & $\frac{\alpha}{\alpha} 3$ \\
\hline 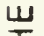 & $\perp \Sigma$ & $\underline{x}$ & $z$ & $\varangle$ & 4 & هu \\
\hline$I$ & 曲 & $\simeq$ & $\varangle$ & & $\mapsto$ & 15 \\
\hline$m$ & 片 & z & & $\alpha$ & & $4>z$ \\
\hline$z$ & $\alpha$ & 4 & - & & 0 & $\infty<$ \\
\hline- & $\leq \geq$ & $\mp$ & 0 & $\overrightarrow{1}$ & $\underline{\alpha}$ & $E \pm r$ \\
\hline & $<<$ & & 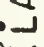 & 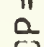 & U & $\leq 00$ \\
\hline
\end{tabular}

Zn

Zw $\frac{\alpha}{0} \propto$

z UUO

जn

रद⿺ $\propto 40$

xis

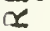

प⿺辶万

<岁 $\propto$ 占

\lrcorner : шठठ

- لس

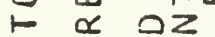

in. $0 x^{2} 0$

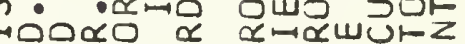

$x<\omega u><\cdot 0$ da

$40004<0>a$ a zunO

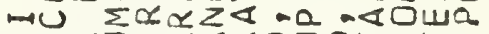

wxग $x<x 0 \supset 0$ -

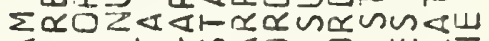

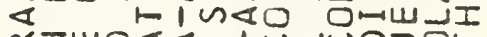

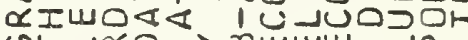

जि

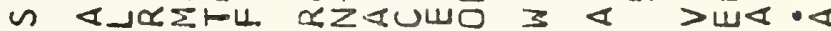

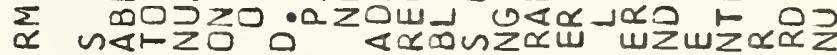

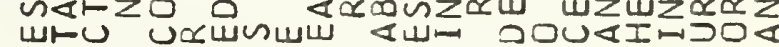

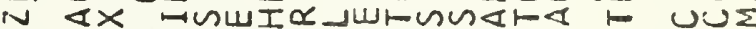

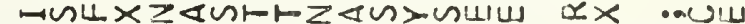

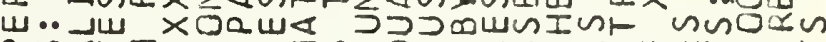
a

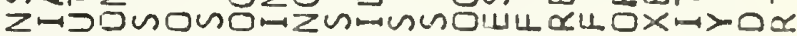

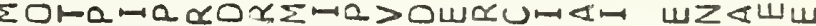

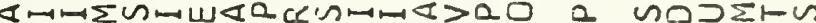
$\propto \vdash z 0 \propto x N w$ wnxomo

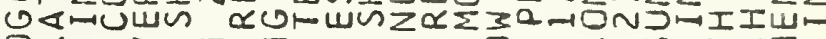

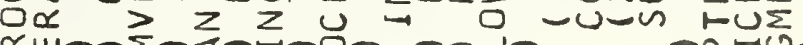

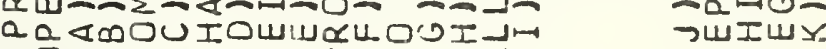

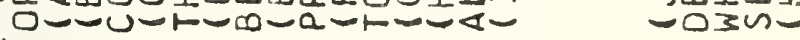
IL

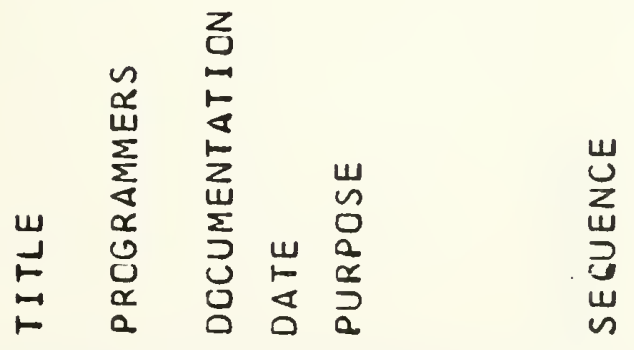

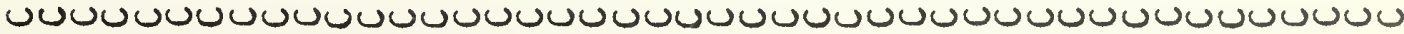





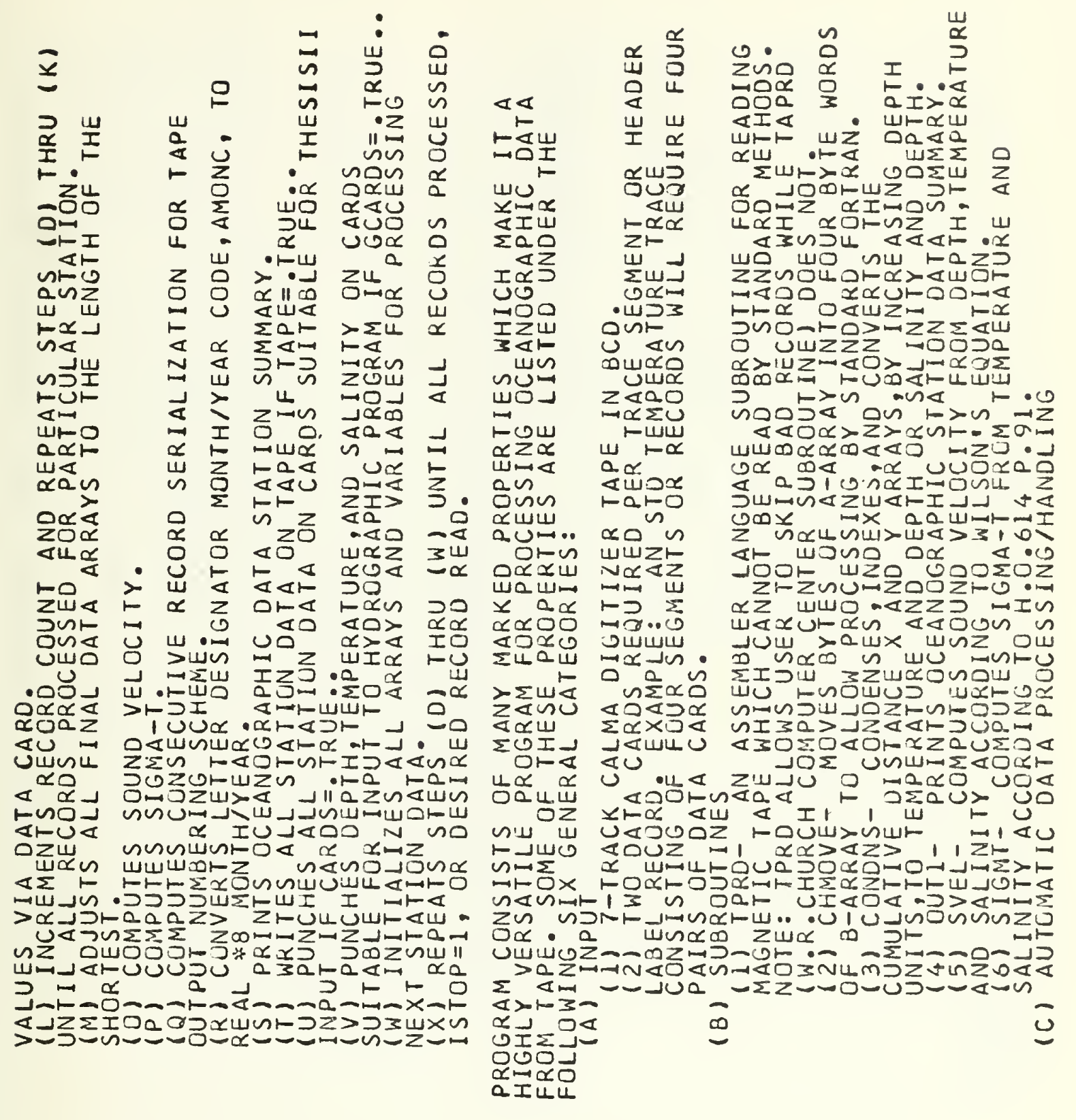

峁 



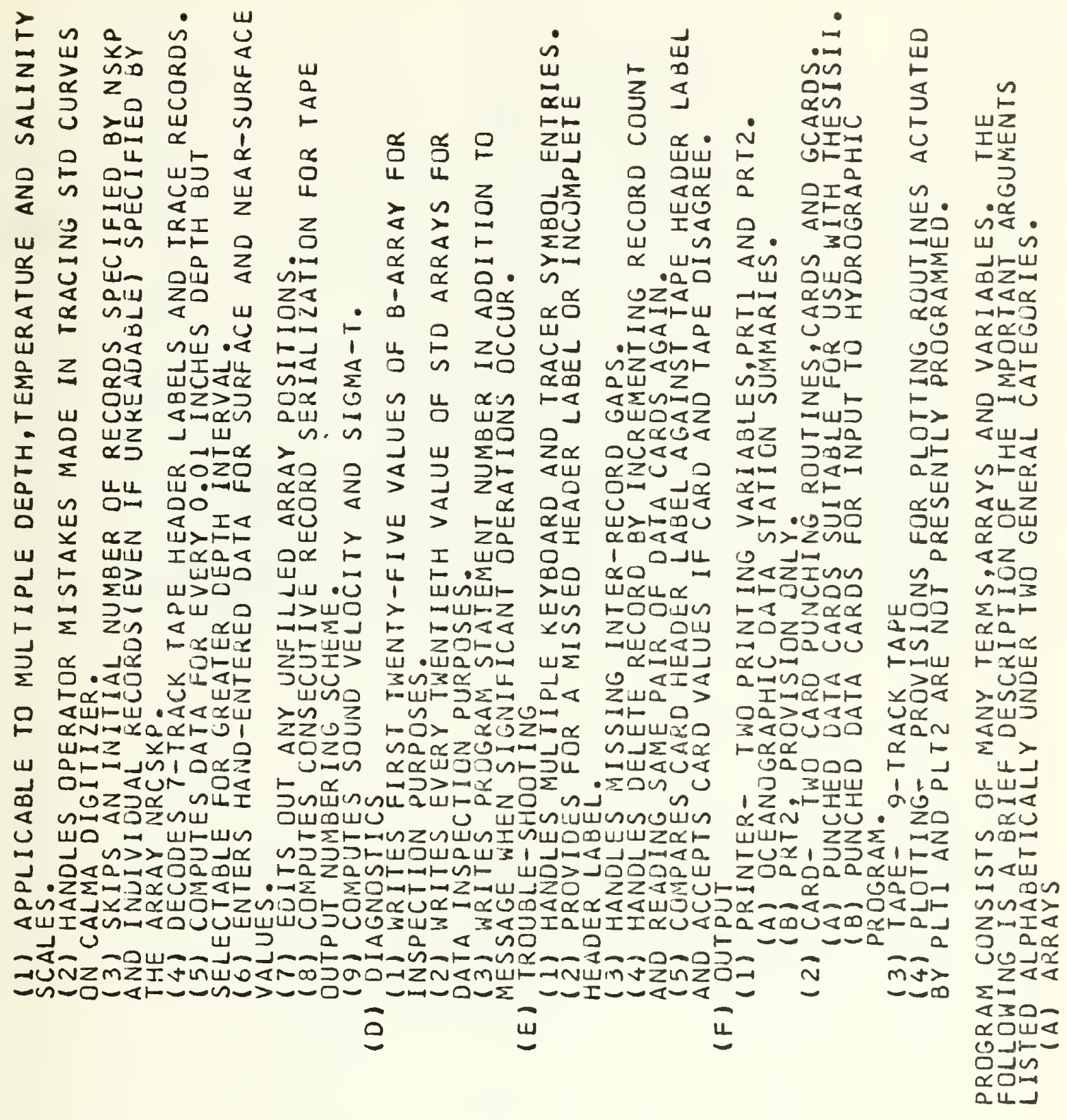

$\sum_{\substack{W \\ \vdots}}^{N}$ 



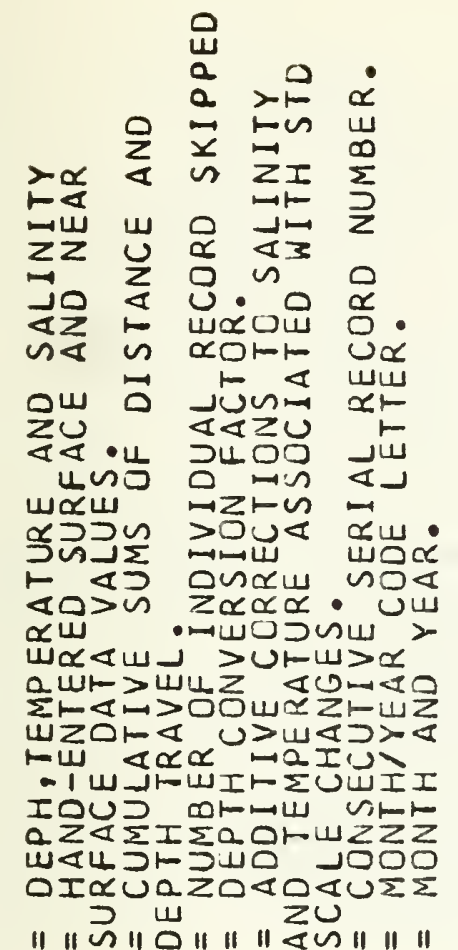

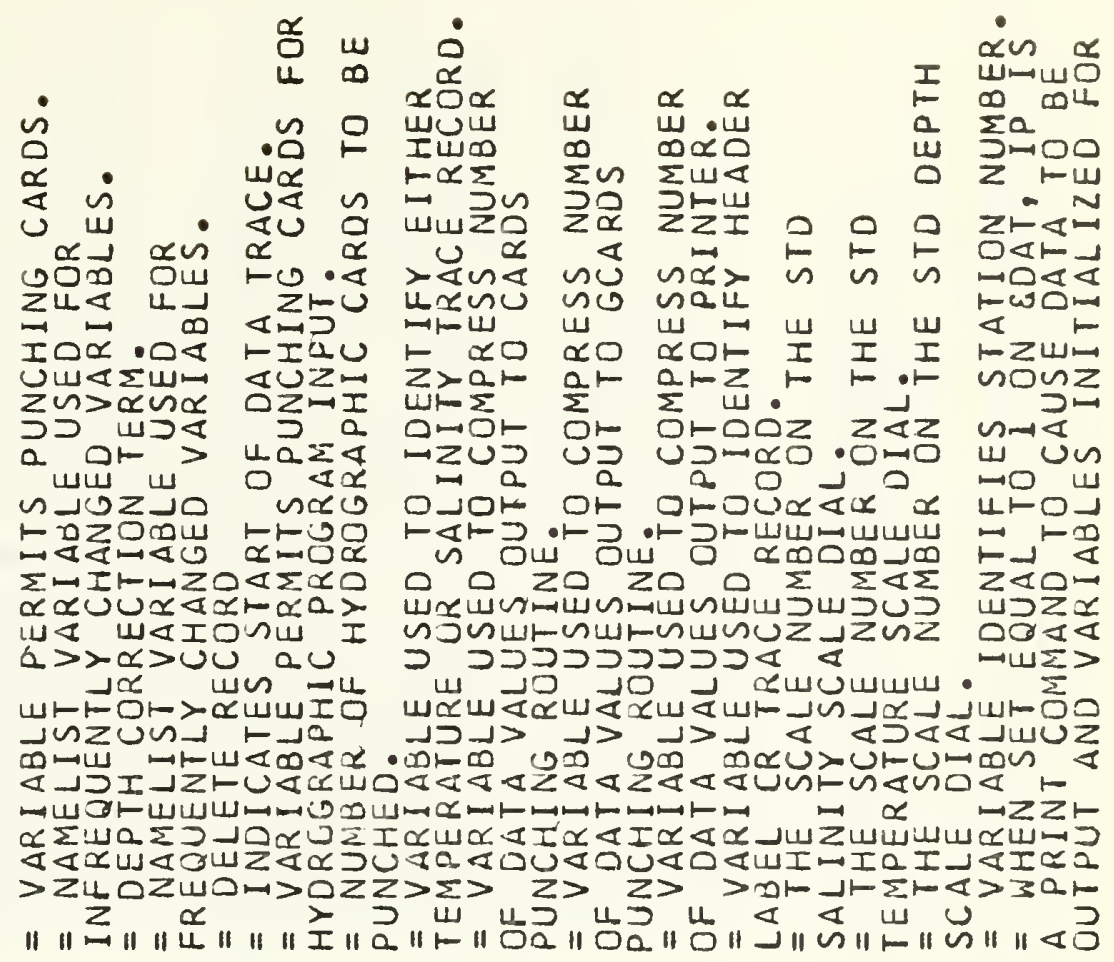
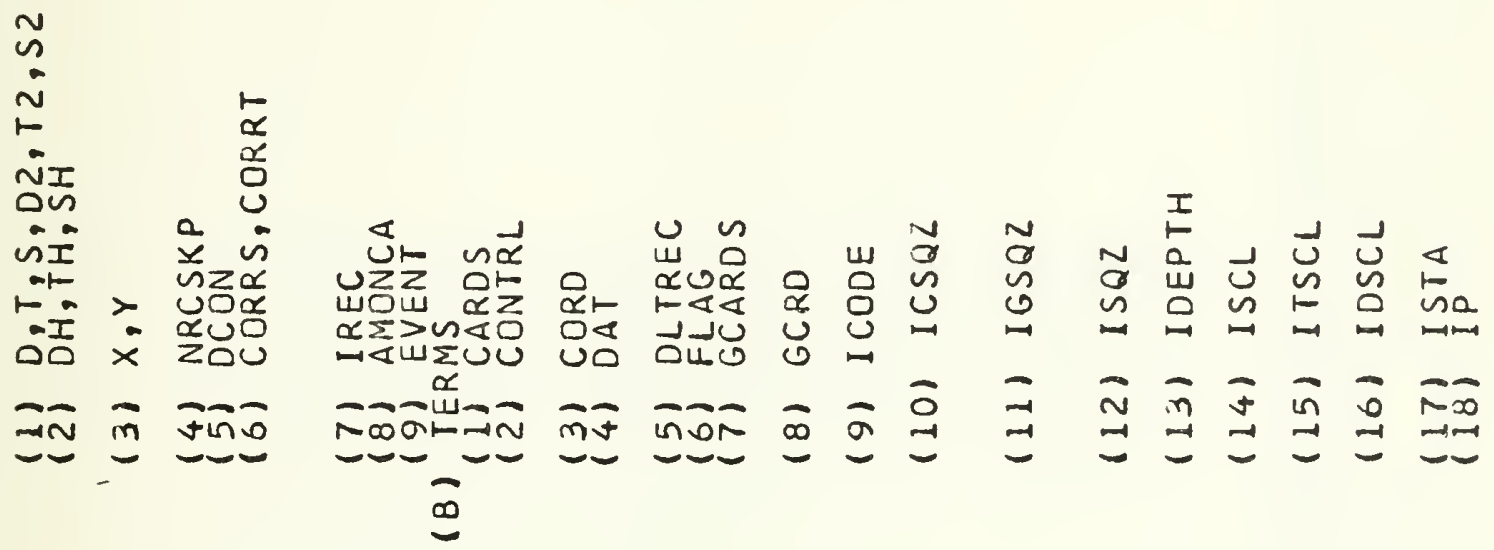



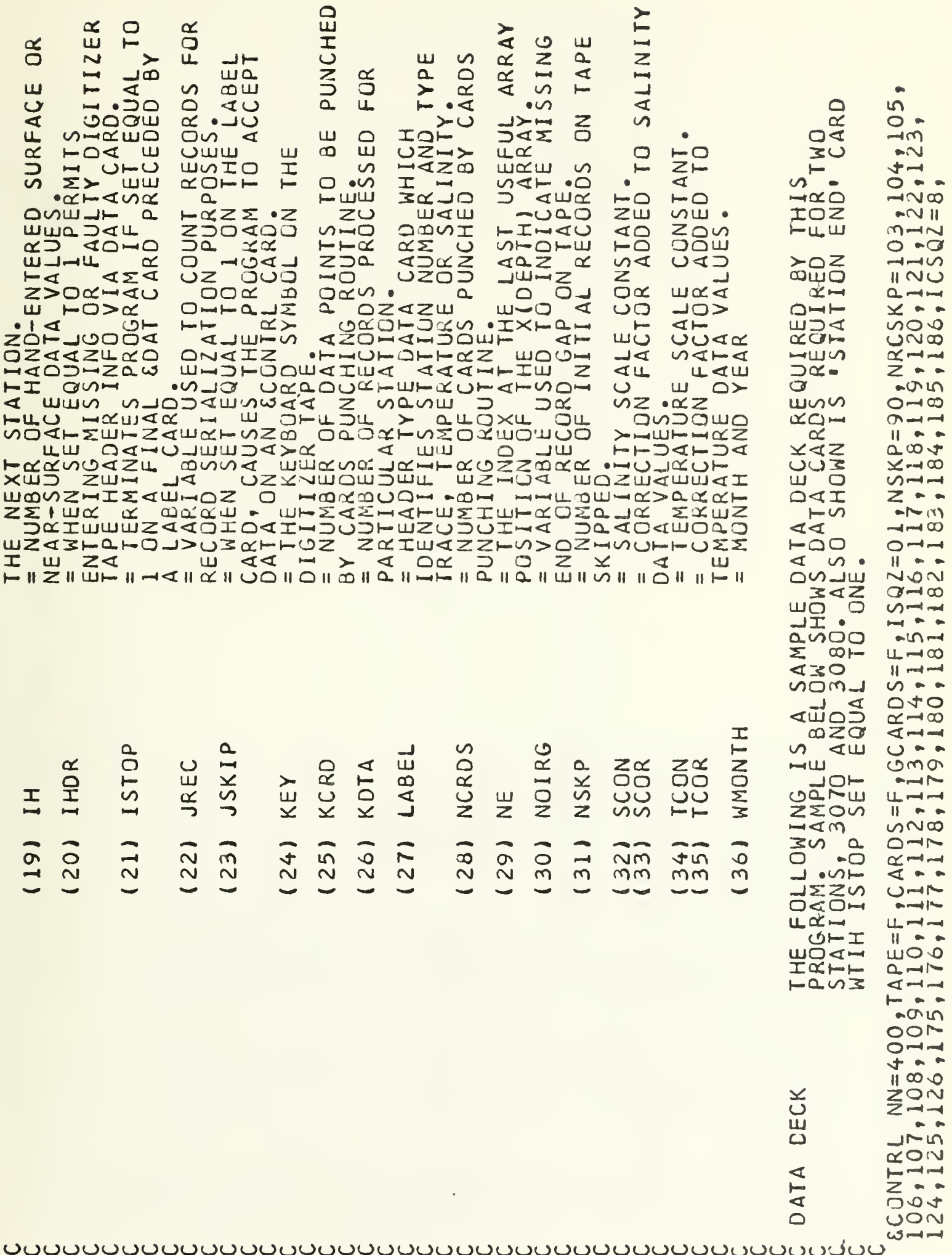

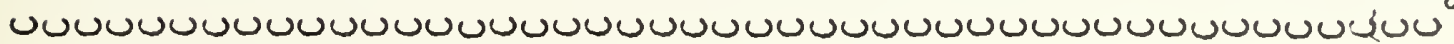





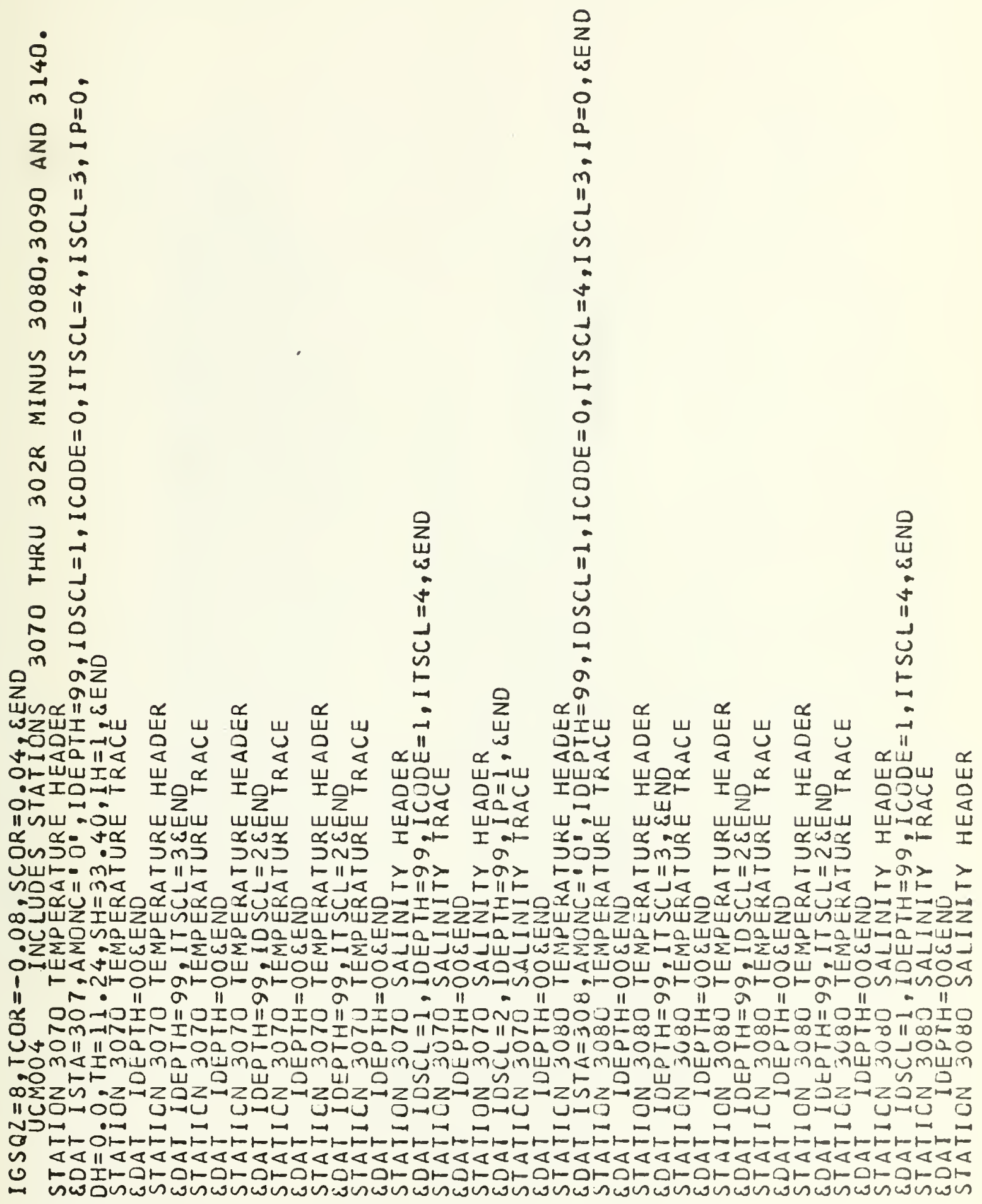





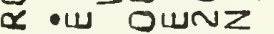

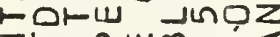

Zトーロயの On $x<N<N 3 I$

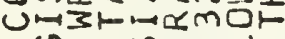

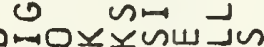

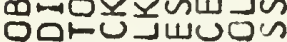
마내네 سox $>$ unutan a oدजा z-0шس गoर Oum wo nox

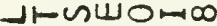
a 4 I t

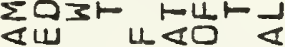
$x$ xo. سי人 $D N, N D$ a Non

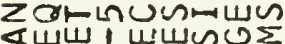

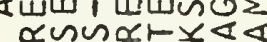
n $a-1] 10$

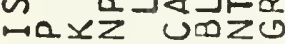
DU $<$ \& $<0$ $01 \omega-0<>\alpha$ ZトดแIZ रง $\alpha<\omega<$

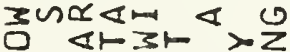

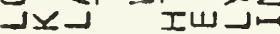
य๐x

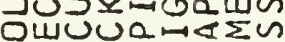

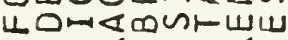

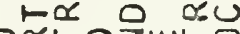

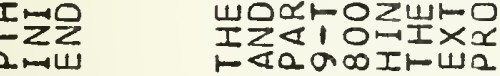

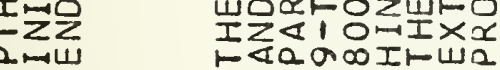

ano

NoI

"DIเ口

Jonzu

บмшा

वे $z=0$

⿶凵

$\Rightarrow \quad m 0$

$<<<<\omega$

oror

wuswos

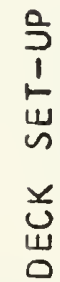

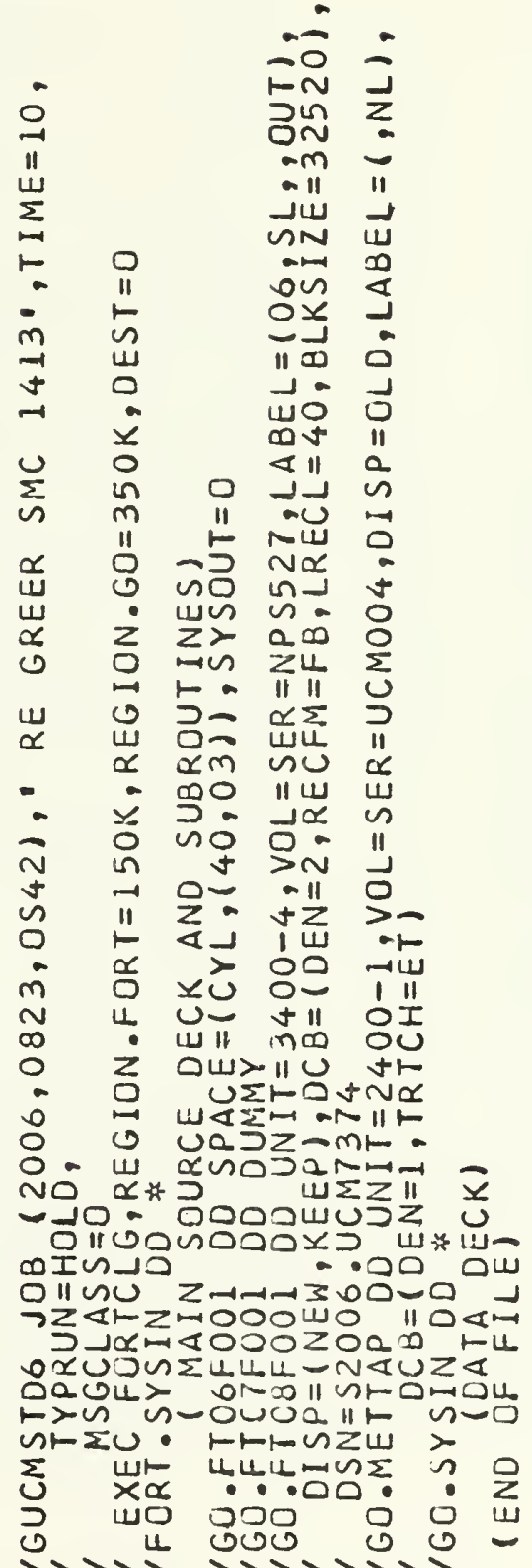

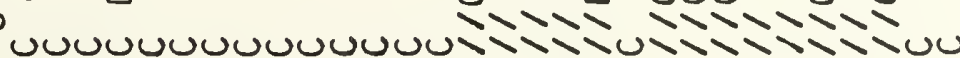





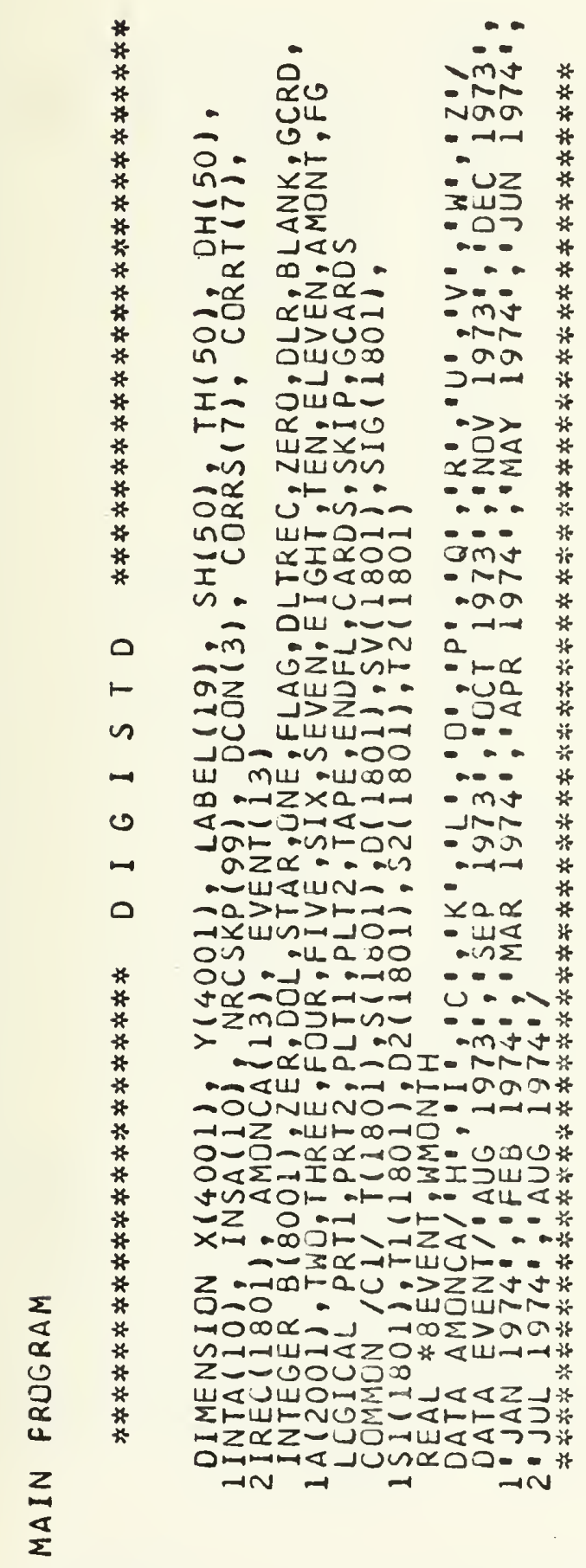

$\stackrel{0}{0} \not * *$

in-

i

요 $\bigcup_{-\infty}^{\infty}$

$-a$

$\sim x N 0$

$\rightarrow 000$

-un.

nuson

D $\rightarrow \pi x$

$x x \sim \alpha$

\&ONO

Uง

arn -

$\perp \rightarrow \infty a$

un a

oDIx

zanu

$4 \leq a-$

-บய๐

แण

․ $\rightarrow 0$

$\varangle a_{0}^{-U}$

$1 \mathrm{Ga}^{-}$

asm

NO -0

$\ln 20$

anus

- 302

니으

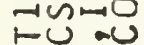

Jindu

$2-0$.

-viz

Non?

- ヘேル

$\alpha \mapsto a$

a. - .

-uva

-1unto

$\alpha>a-\infty$

a - - -

-a oud

$\sum-0 \propto$

Zxun

ง $\rightarrow 20$

1?-z-n

aㅔ $x 0$

$1 x$ a

ZnII

$020=0$

QII $2 u$

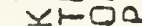

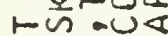

nu다

任的.

구이

ए

$\sum \pi \varangle 00$

$z-1$

trint
荅艾

范荅

*

前

话

落

话

$x$

* 话

* *

却

*

落

艾

*艹

$x$

谟

* *

次

*

*2

纹

的

炎

话

之

$*$

范山

$* 0$

x

*

荻

\%

解

is मे

范苏

* 证

if 许

सै

*ै

苏

if

*

कि

준

放

希角

获

获些

if 然

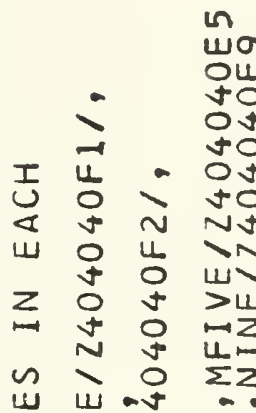

Z ZN

$>\quad 0-1+\infty$

-09

40 15450

1 10.000

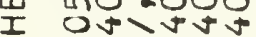

700000

แ OงUナง

II TNOONN

I ONAt)

InNuSNar

z>Zலแ0ல

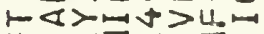

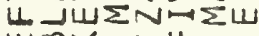

$4 \infty x-1$

I $>$ OH $\mathrm{mN}$

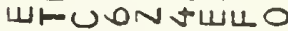

Im $m$ in - LOO

- $307>0 \pm \pm 0$

$+0 .+001$

hol

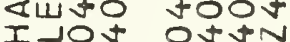

- JAN INN

MN $N=N Z$

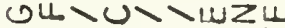

$z \propto \alpha u \simeq \propto u>$ $=0<\propto \geq \supset \propto>1$ 0 $n-14 t \infty u$ $z 0 \cdot \overrightarrow{0} \infty-\Sigma$ $-Z \backslash--\infty-1$ जய- >m>00 - $O$ ODUNUL Dominomoo Lu $0070 \pm$ $>0.0000+1$ $n> \pm \pm 0+00$ - $70000 \mathrm{~T}$ யZDオナNホN ZUDNNVNV

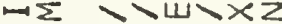

$4 山<0 x: 40 \omega$

너 J

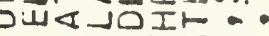
aunm $\mathrm{m}=$
*

* * $\alpha$

*

* $*$ a

范心

若菜

if $*$ IN

* * Un

if $* \alpha$

艾

茫落

* $\sim$

* I\&

$* * 10$

论

*

茫

*

$*$

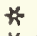

* $\backsim$

踏

it Z ZZ

* $<$ ㄴ

类

2

前忌

*U 供

*世

诺 Z

范四

*U U山

나

* 0

*

茫些

论 论

艾 3

话 强

艾关

if 获

if

话

*

貉

if

से

象

if

*

头望

识

*ै

if

获

if

*

4

2

$0 \Sigma$

ज)

๔u

UI

2

U.

-m-

(2) ก 0

Zc-n

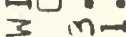

Dn

- II II

14

$00-0$

$1=$

$4 \div \geq 2$

- 50 



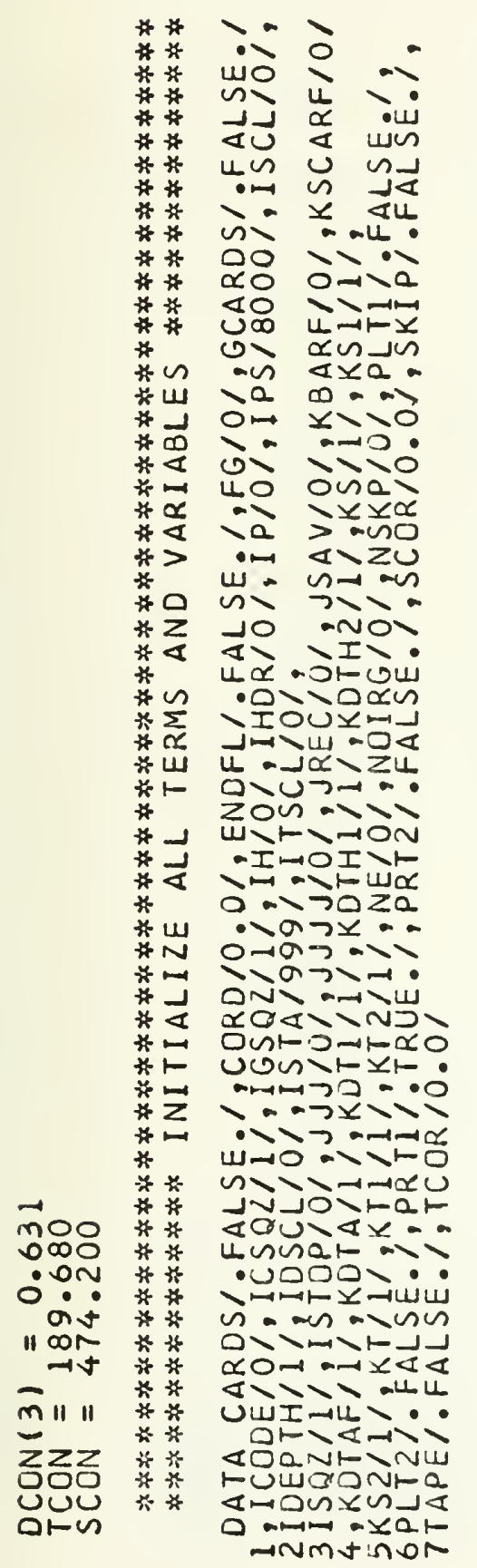

טuט

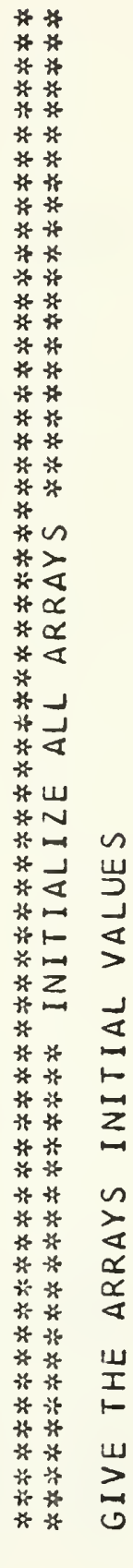

잉 no.? $\rightarrow 0 \ln 0$

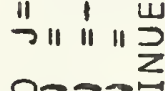
ำフラー 드 QIIIDU 우

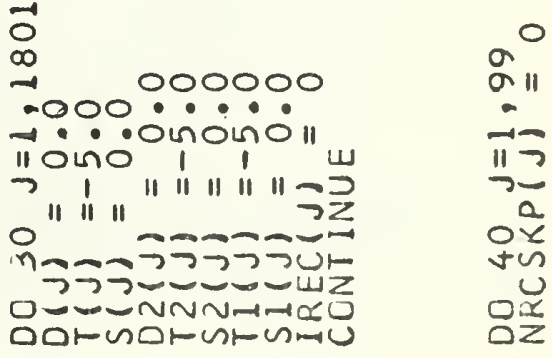

m 



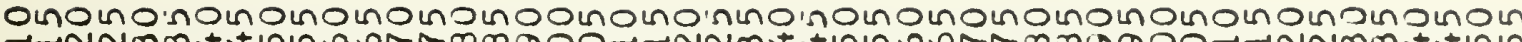

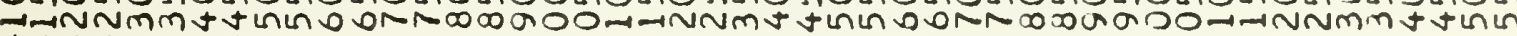

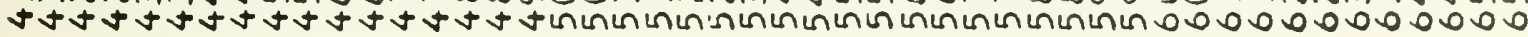

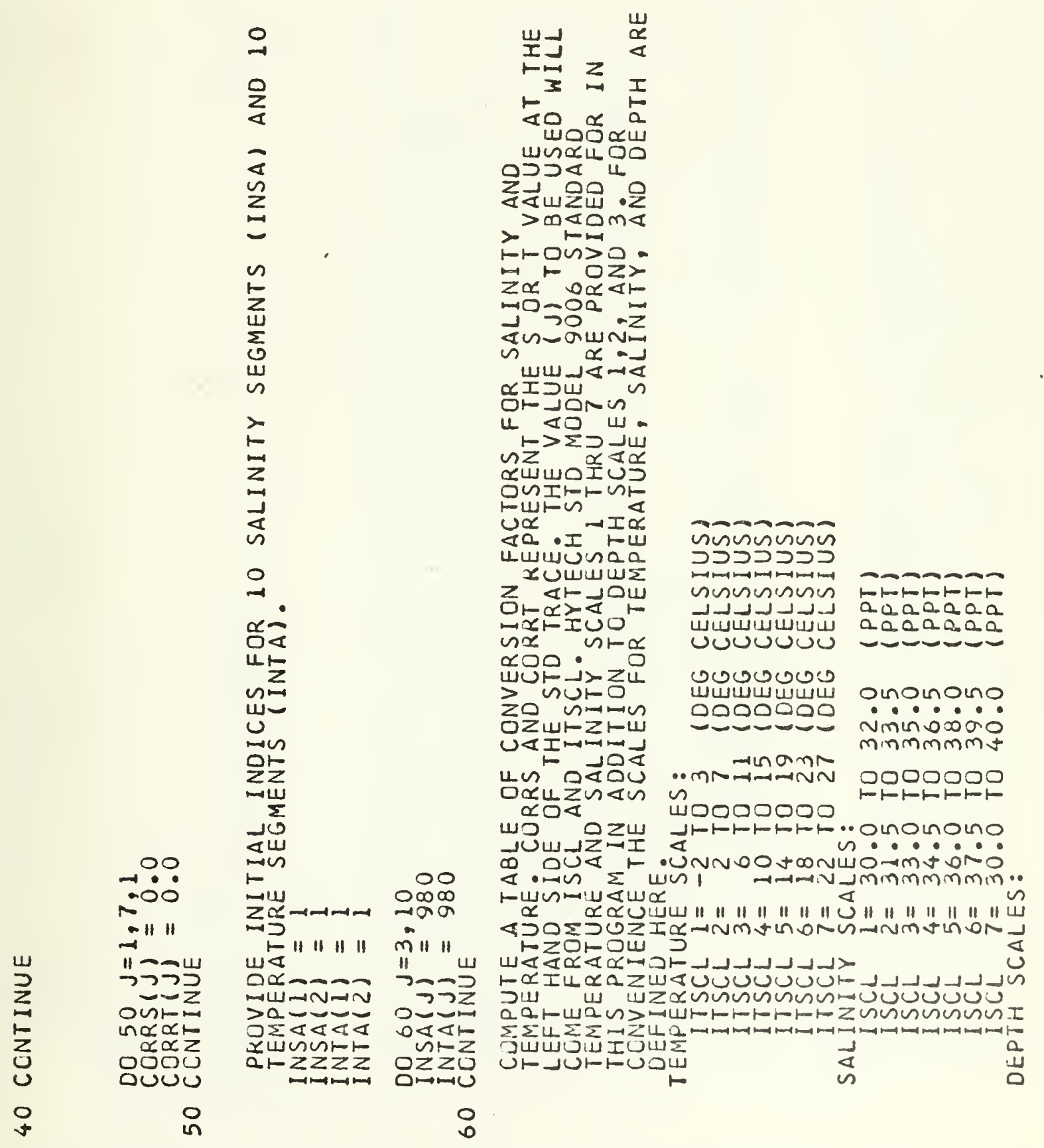





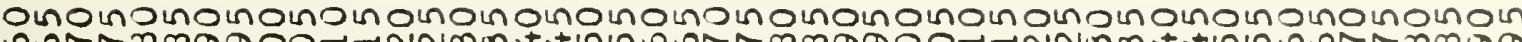

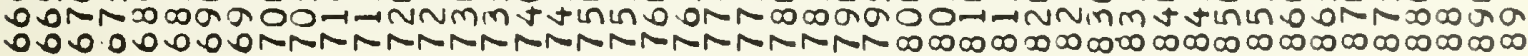

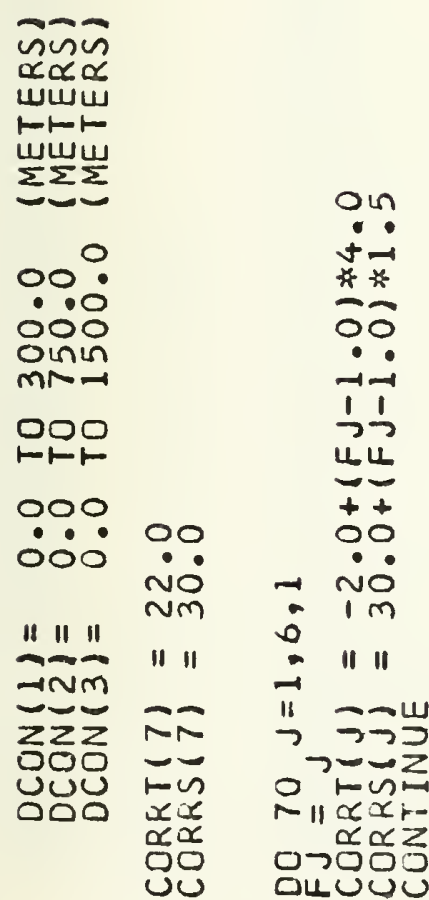

○

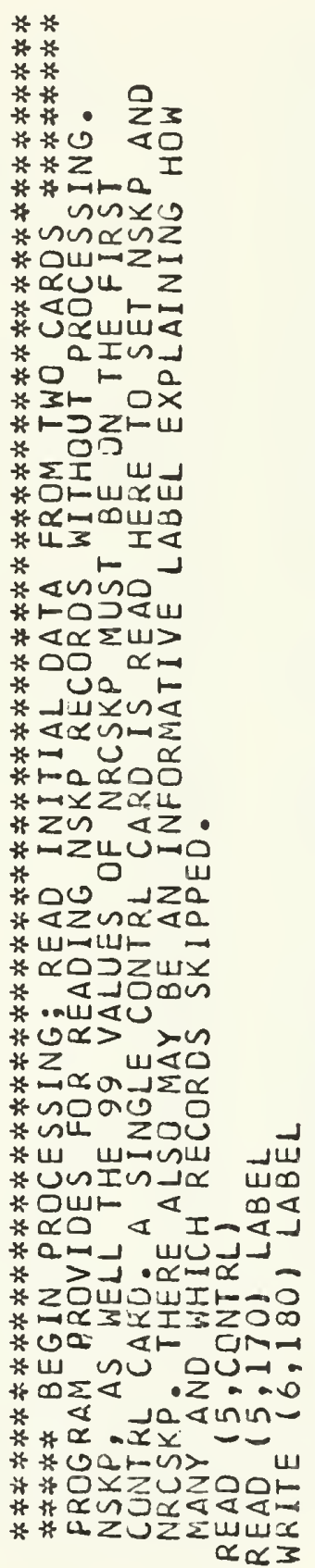$$
\text { (⿸丆口 }
$$

\section{}

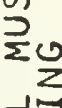

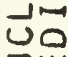

$\supset$ U⿺

แயบ

$\vdash \propto$

$\varangle a$

$\propto z$

อำ

뜨

0 a

$\varangle \sum$

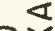

zu

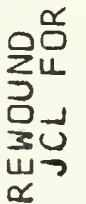

$\propto$ wz

แயน

$\infty \sim n$

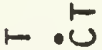

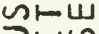

$\sum$

แ⿰

政

a

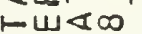

0

-口யz

U⺊ $\sum \sum 0$

๙०णี

யㅣㅇㅛ

-

II $\rightarrow$

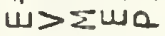

QO OTa

$<\alpha \subset<n$

-a or Z

uய

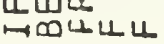

ロル

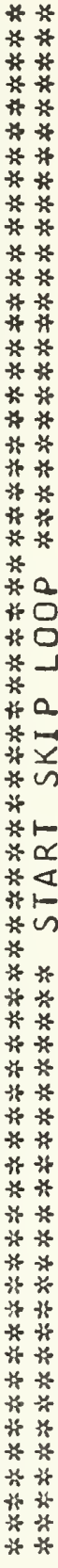

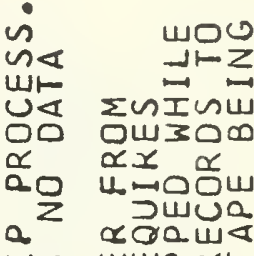

$\rightarrow$ - wயa

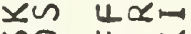

जo 는다네

QS 달

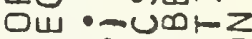

Uana@ do

แ $9 x z 0$

roxn

प्Zに wou- $\cos x$ I

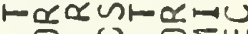
。 $0 \simeq z \cup \sum_{x}$ zயயดюயय 근 aㅏ잉

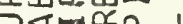

อ0x x⿺辶一 पunous шس แU⿺ $\mathscr{x}$ F 

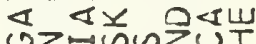
oZnजuzUI யо口 On $z \Rightarrow x<$

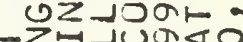
Un

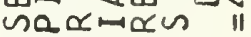
ค유 $\vdash$ -

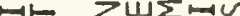

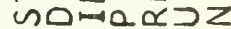
山 वயव जox 매. $a \alpha+\cdots x$ व

2 "a.jung 안 Z5 ш سㅁㄴ잉 Jinozj ze বru- zonu

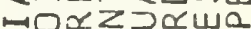

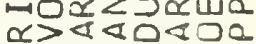
वरा $>n \alpha>\quad x$

OII noOn<ow

$\infty \quad$ w口व

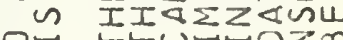

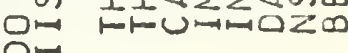





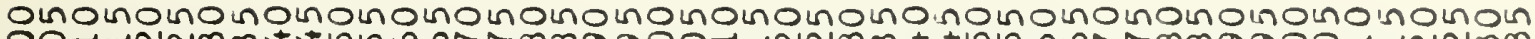

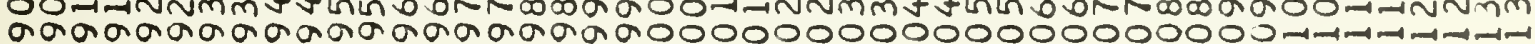

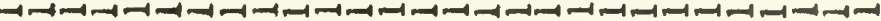

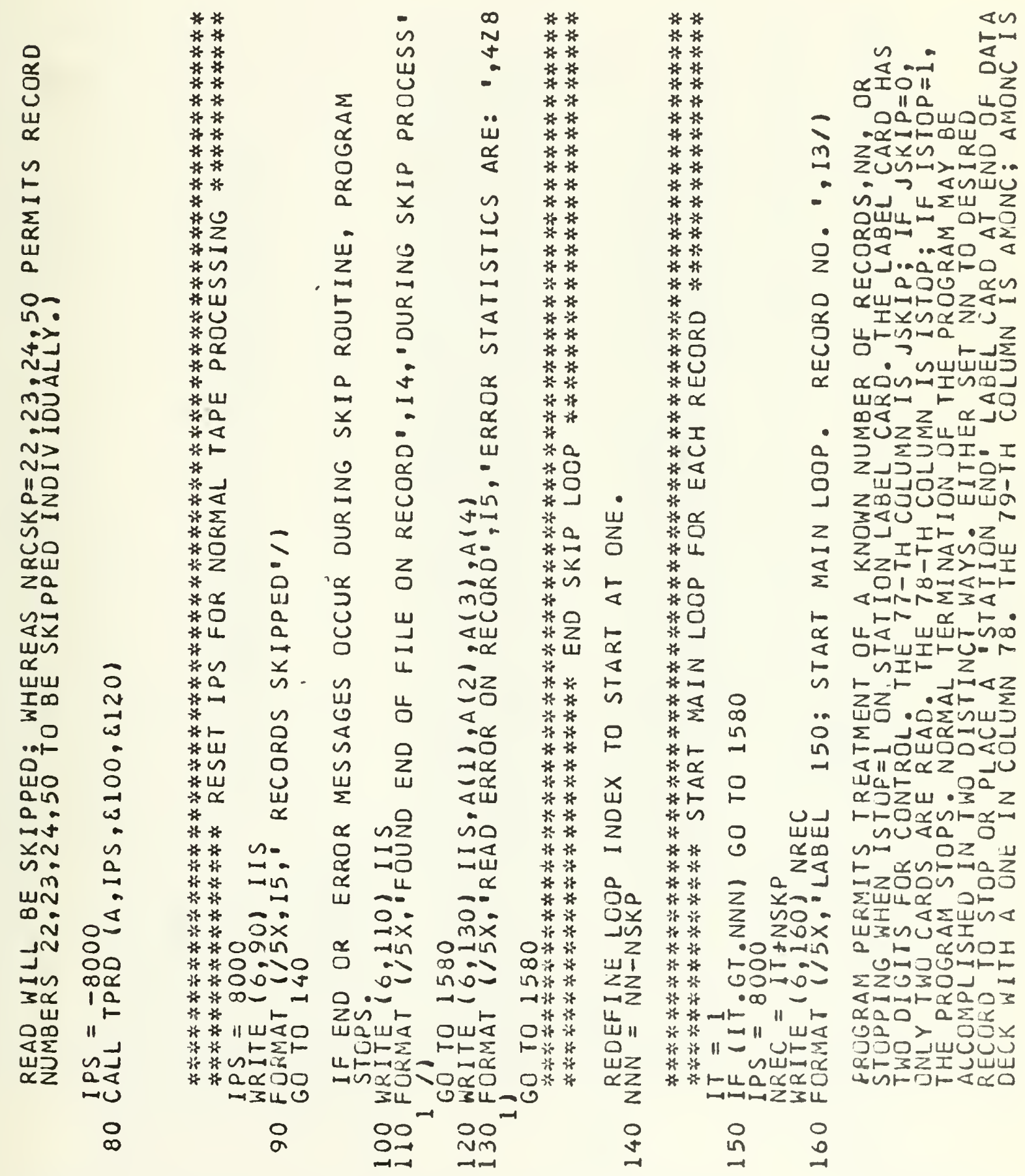





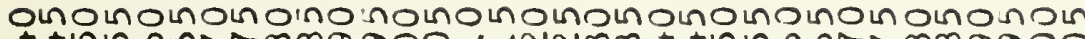

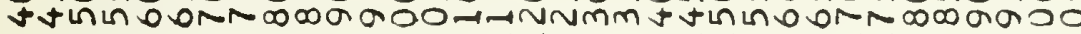
$\rightarrow-\rightarrow \rightarrow-1 \rightarrow-1 \rightarrow-1 \rightarrow-N$ N

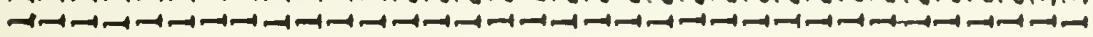

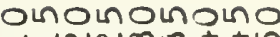
Hinnminttin inmmminmm mm

min

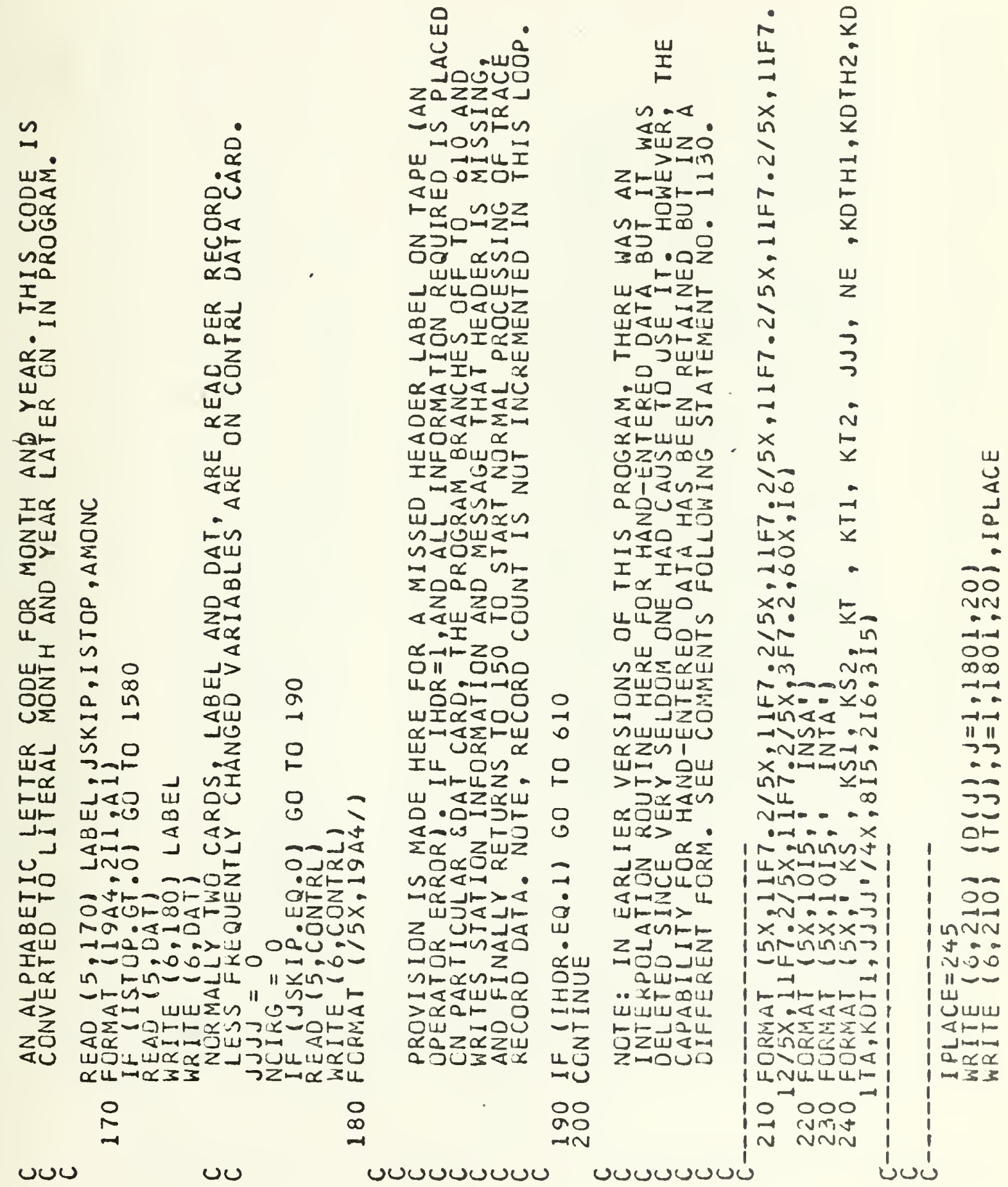





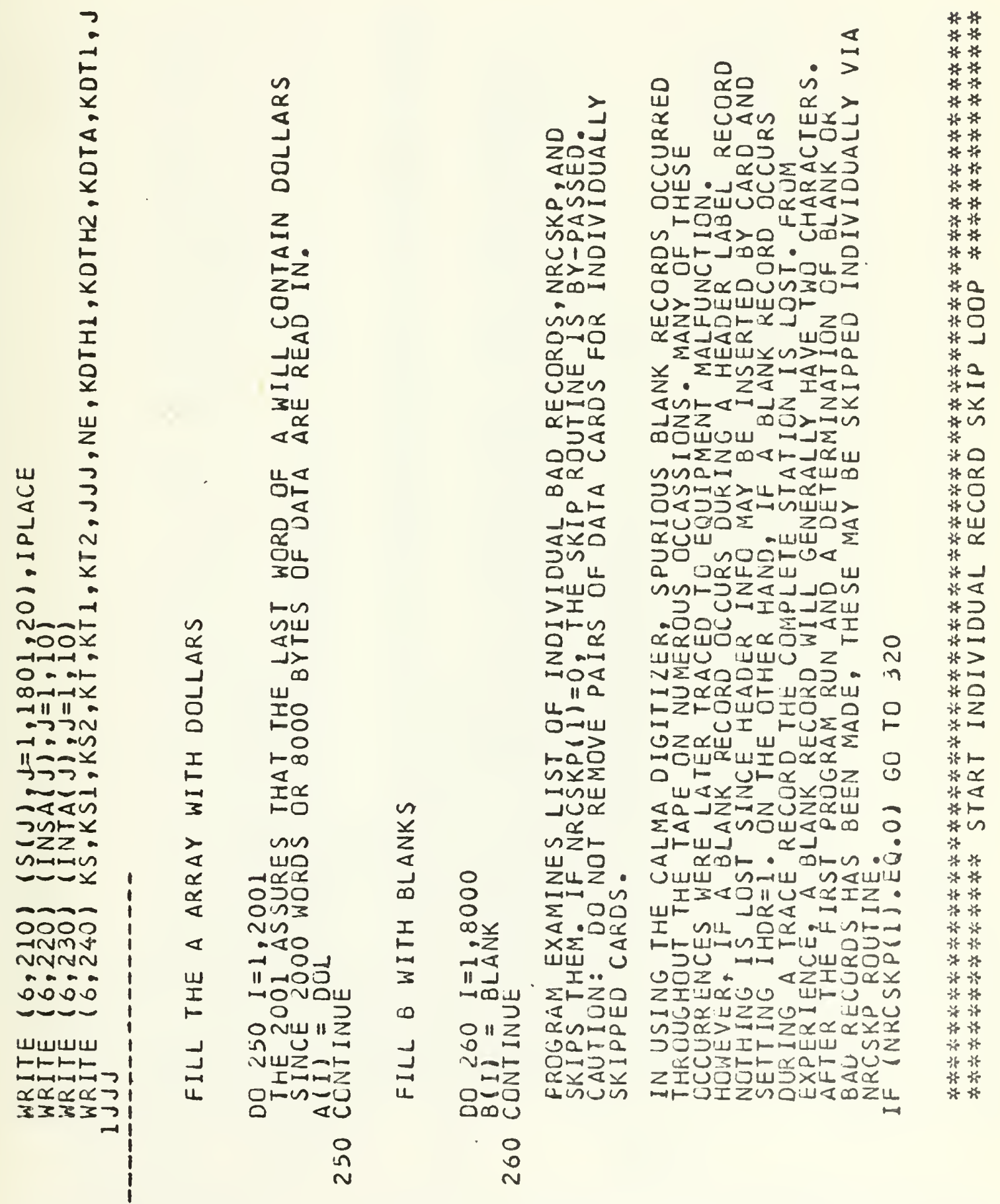





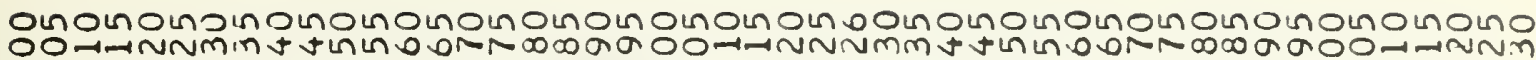

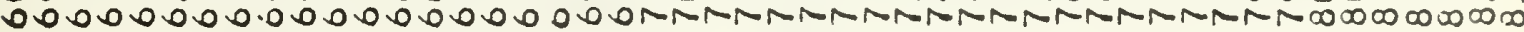
-1 = -

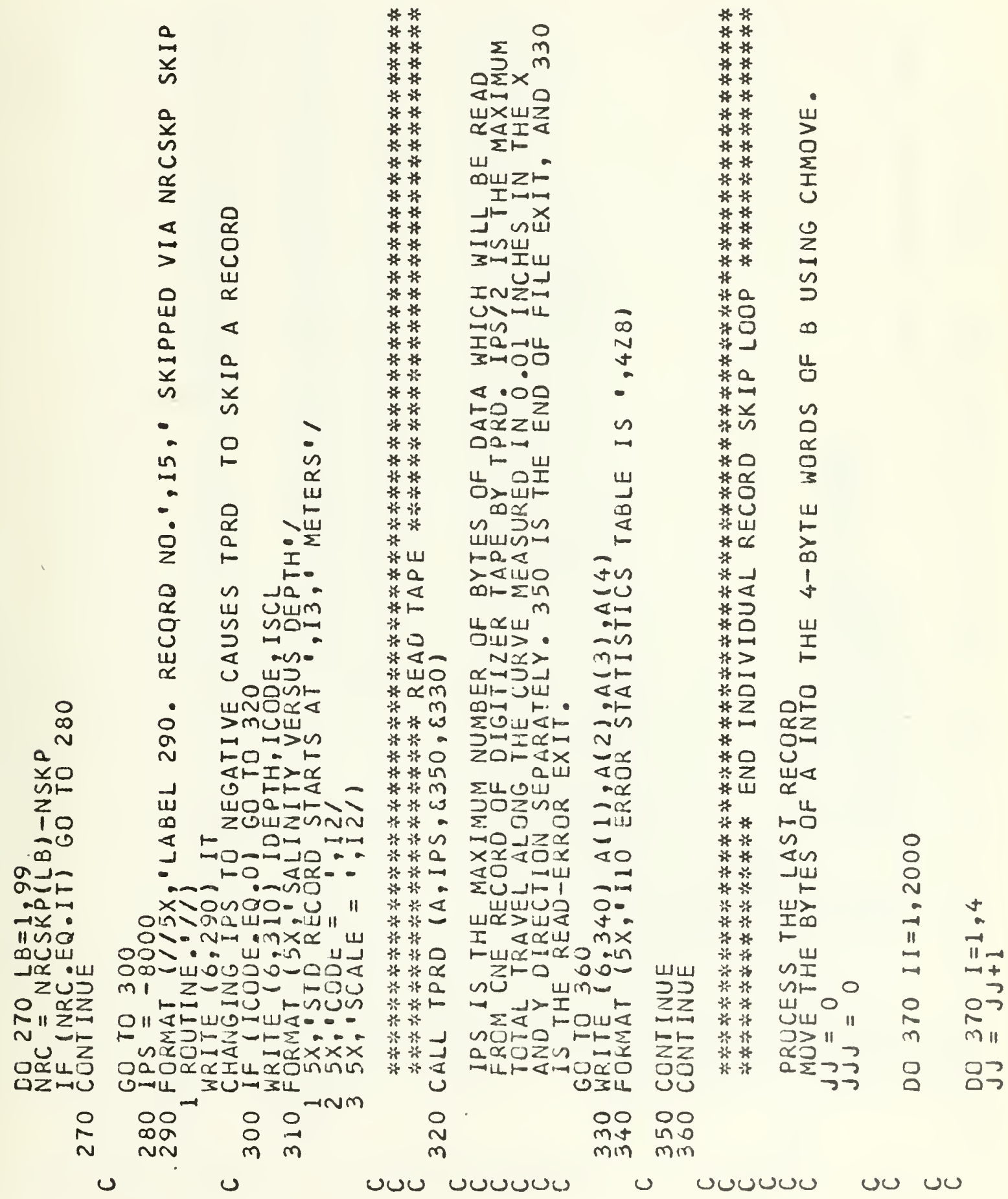



inginounounounoinounounouno

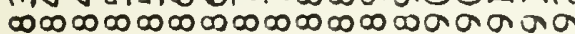

inolno aráa uounoungunounounoun varnaguanajao anaaguananao onolnomoinon

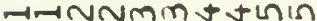
OONONONONONO

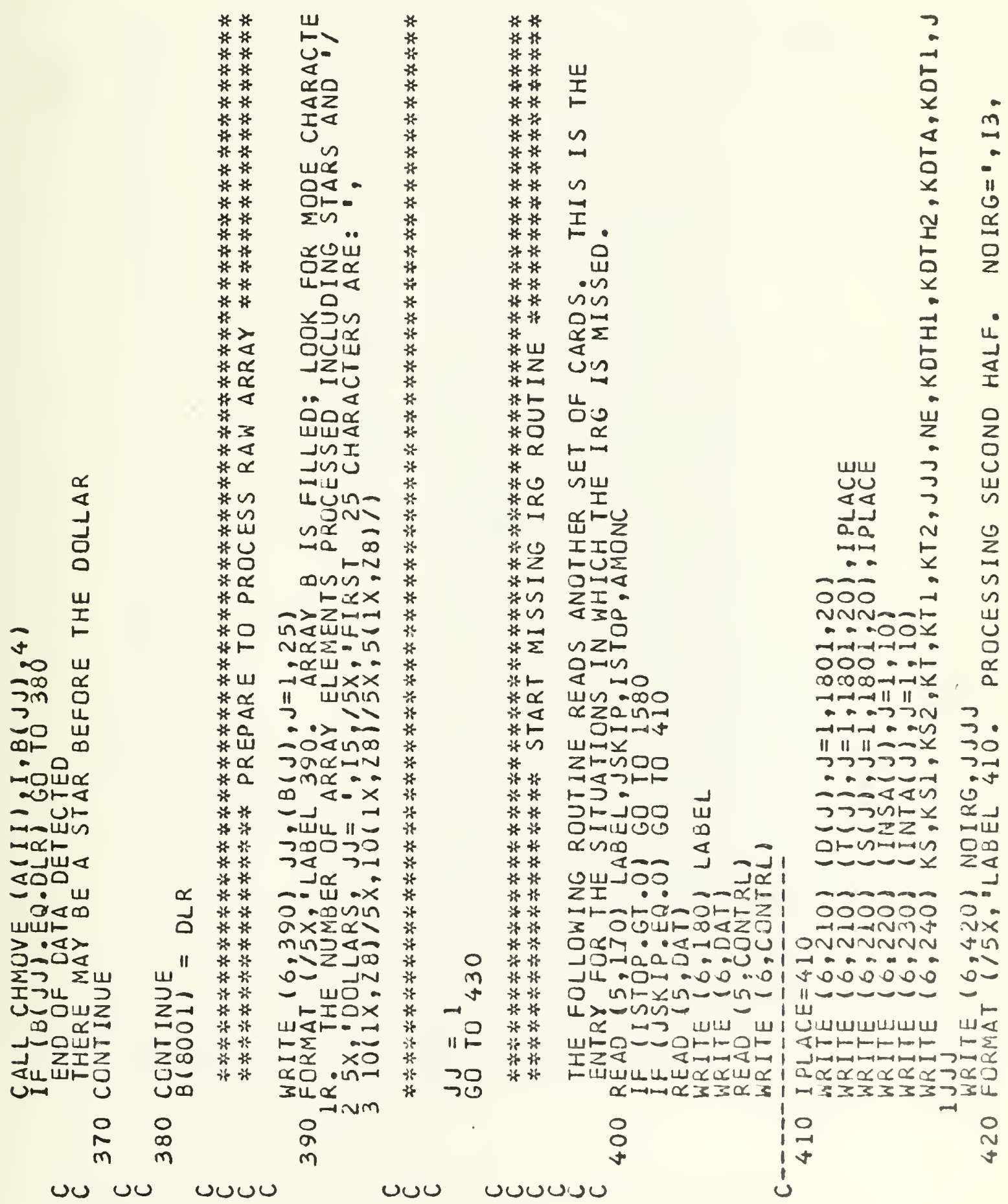





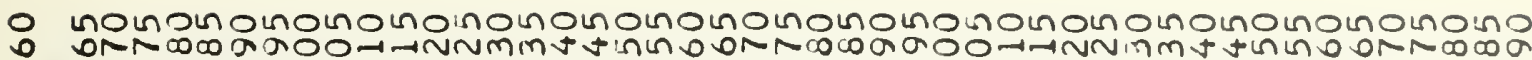

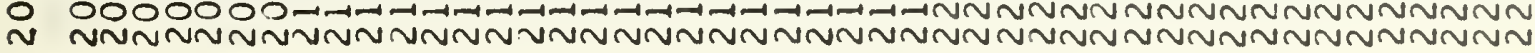

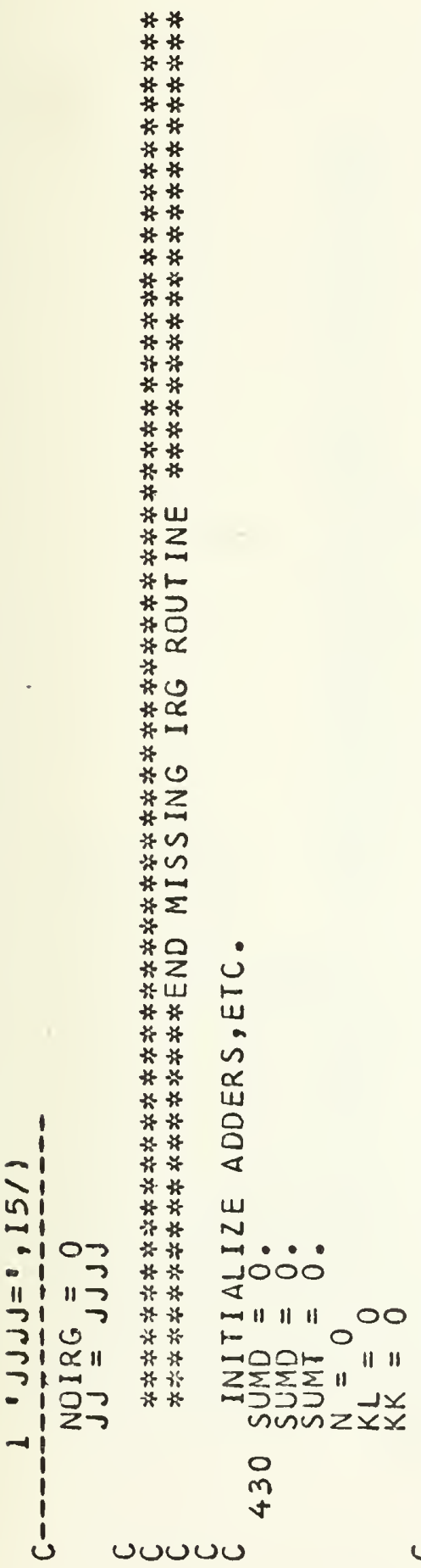

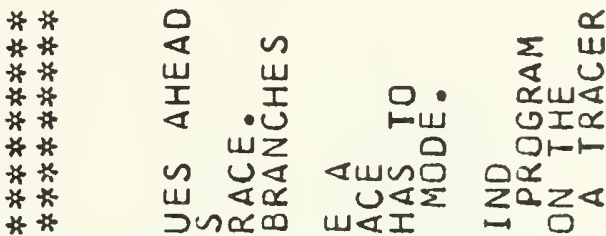

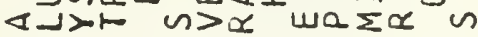

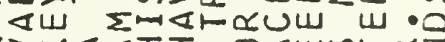

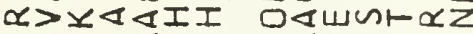

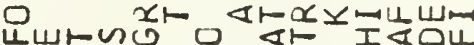
$00<0$ - $14 \Omega$ a

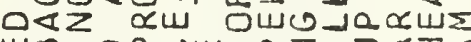

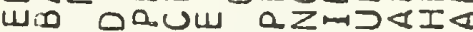

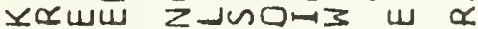
u⿺xu nax $\simeq$ na

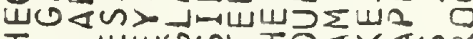

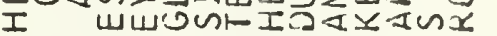
Uயnux जut $x \leqslant$ षQ⿻

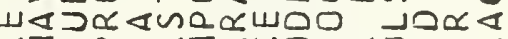

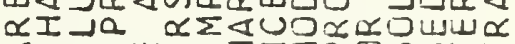

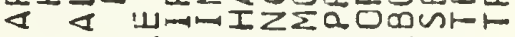
O>nূU Un 2ト $\mapsto 4$ is now

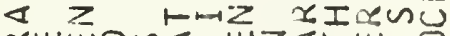

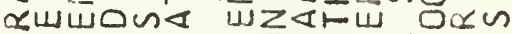
$x \rightarrow>\simeq W \mapsto>0$ \& $1 x<4$

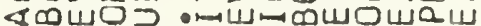

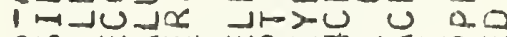
の งயய is $x>0 \leq 2 x<-x \infty<n$

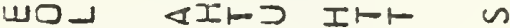

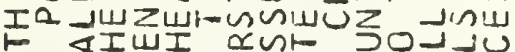
ज $4 I M I$ a OU. 90

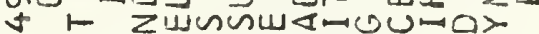
n ow $\rightarrow$ w <t ma $\alpha$.

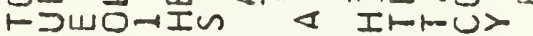
dUD

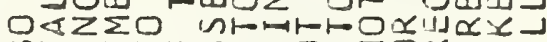

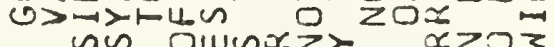
$a z$ t

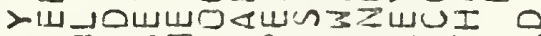

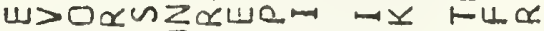

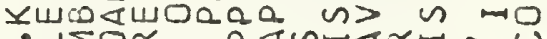
वய>क U०ब ш ज>nแ •レ

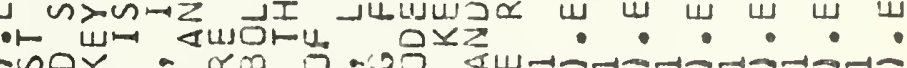
$\neg x x \neg x-\partial \sum \cdot x \geq \Sigma<I I+7+7+7+7+7+7$

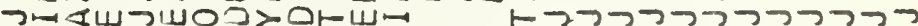

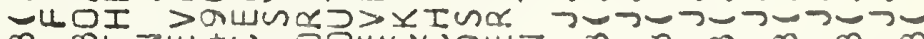

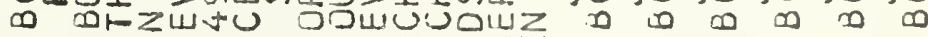
-U> IULI

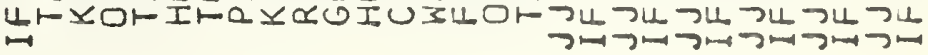

i

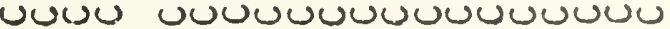





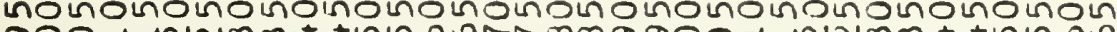

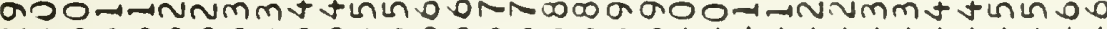
Nminmmmmmmmininminmminm.nmmstttttttttttttt

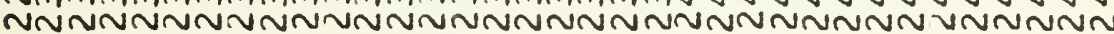

OLIOLIOLINOLIOL

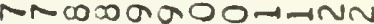
tatatatininininisin NONNONONONONON

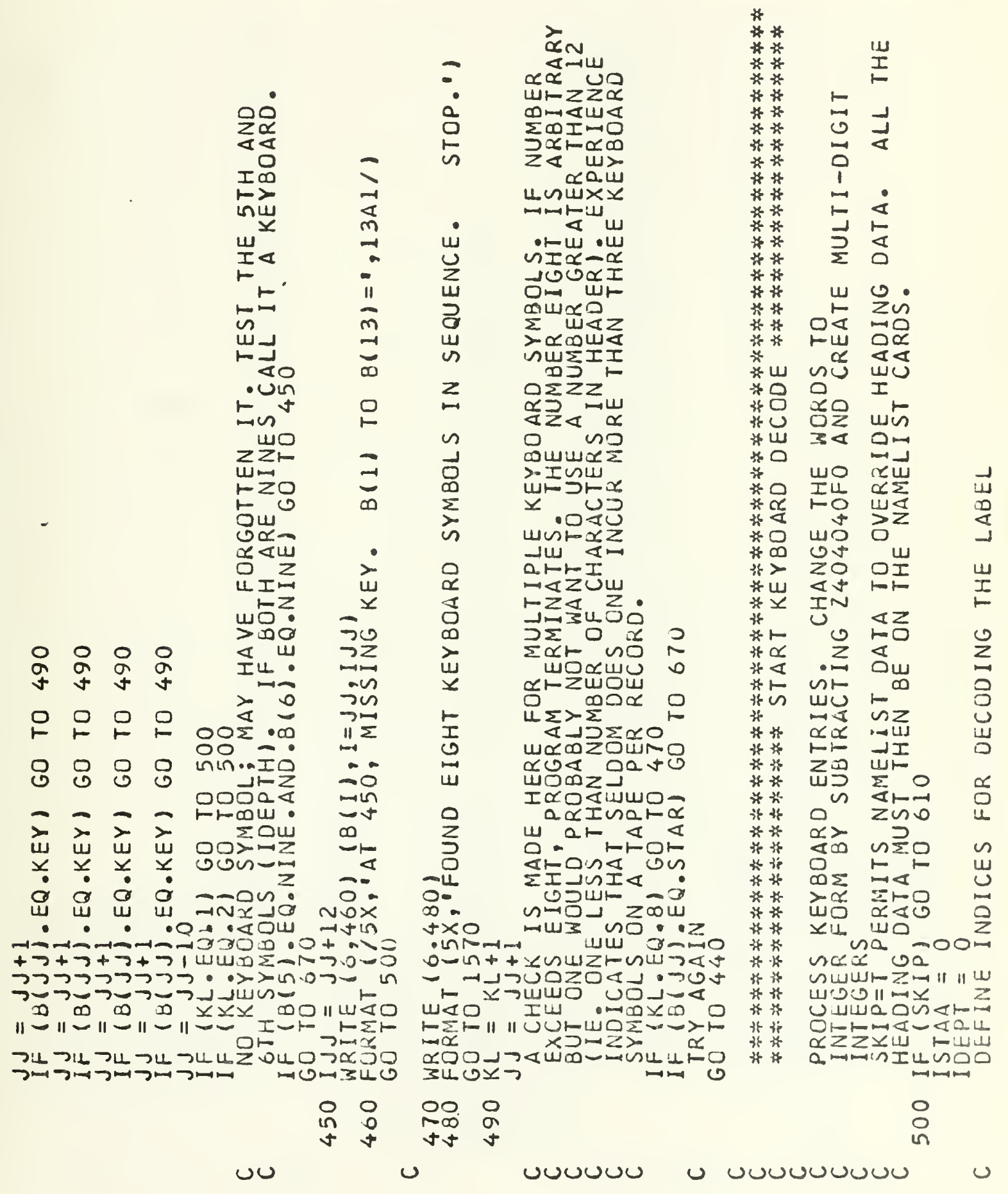





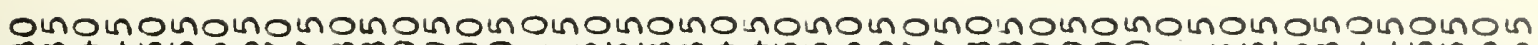
mmt tinL DOR

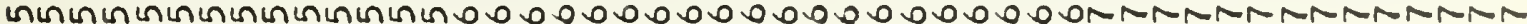

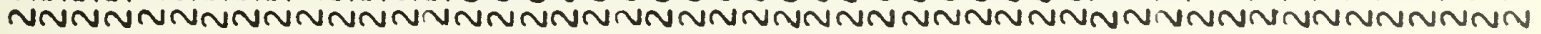

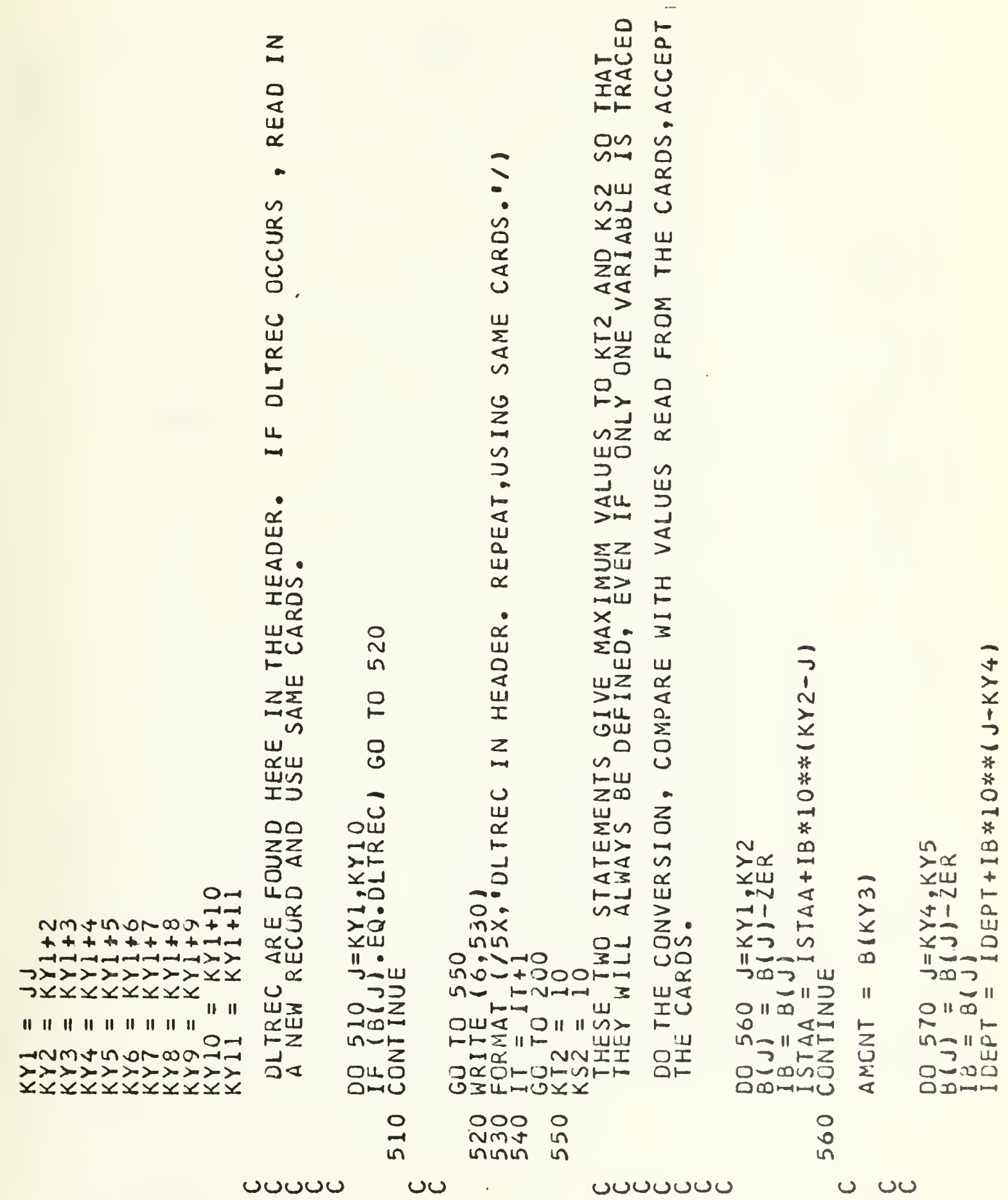





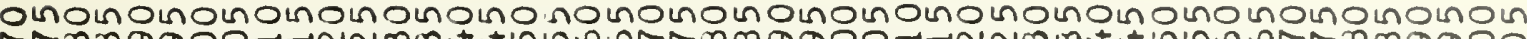

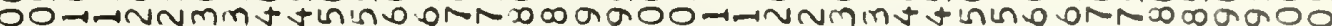

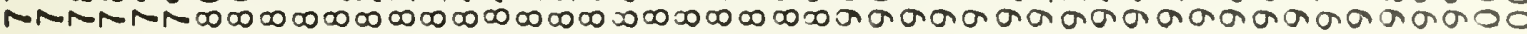

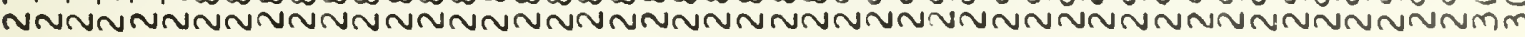

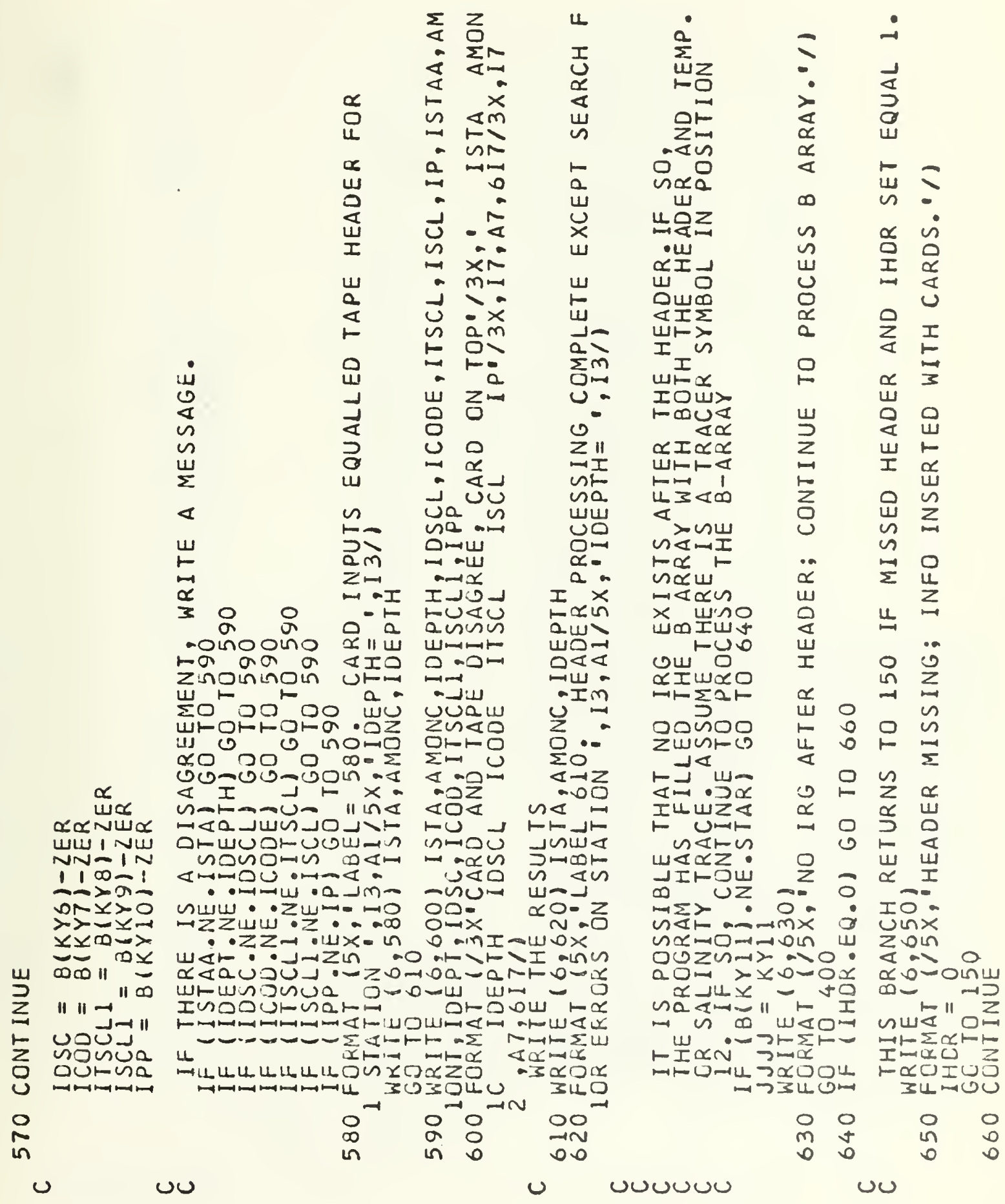





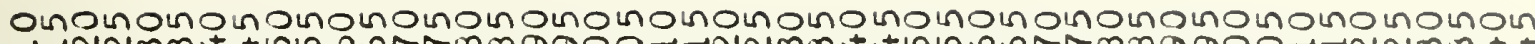

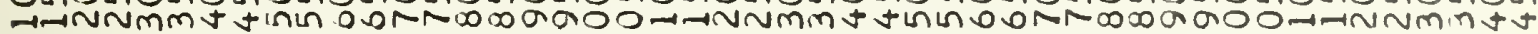

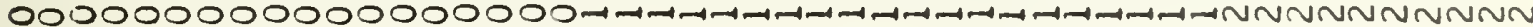

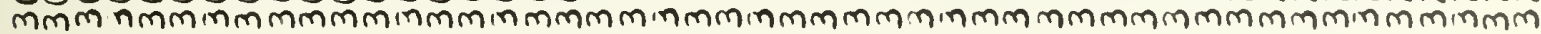

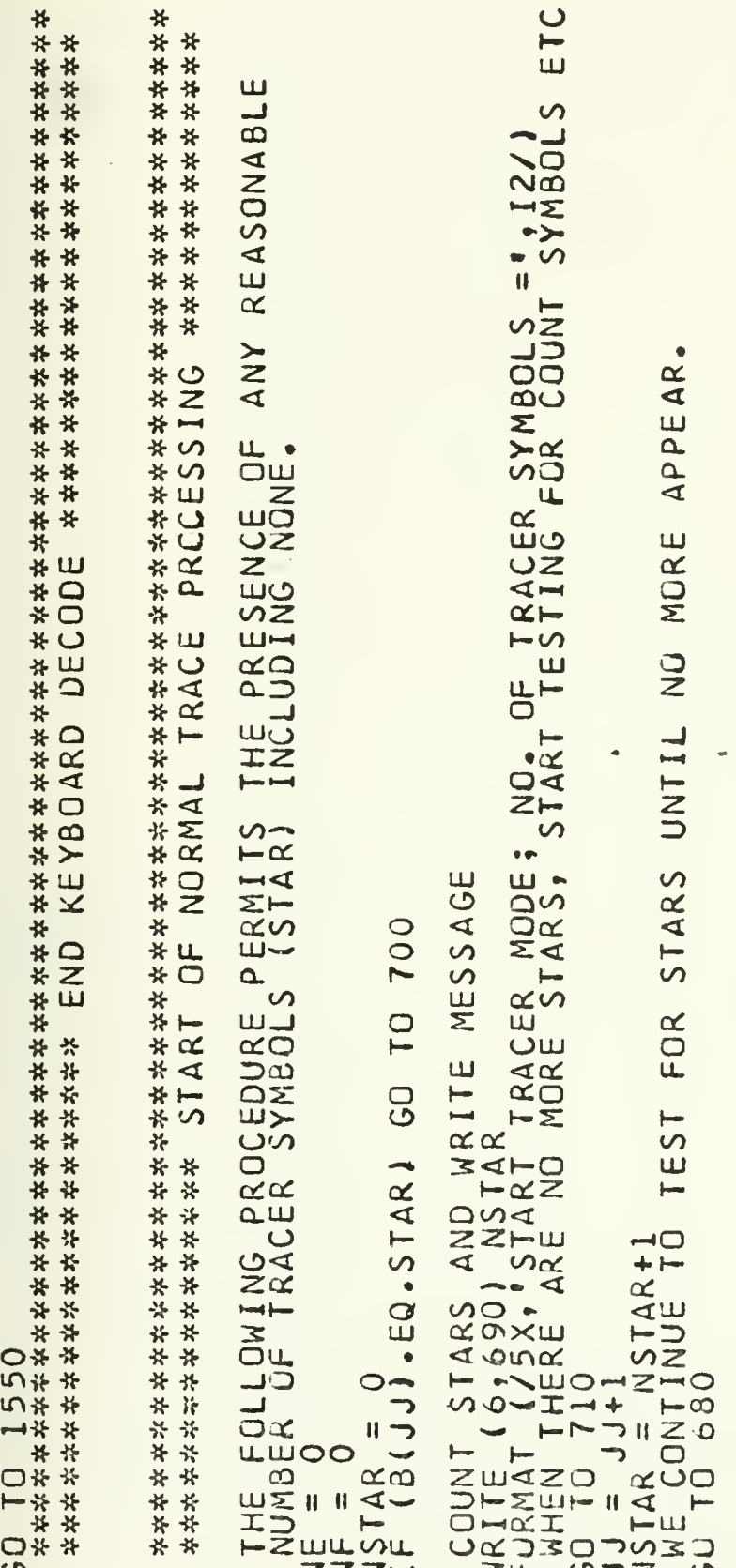





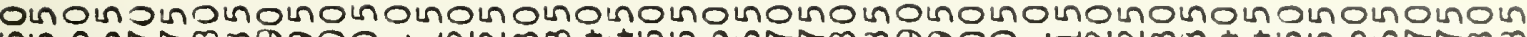

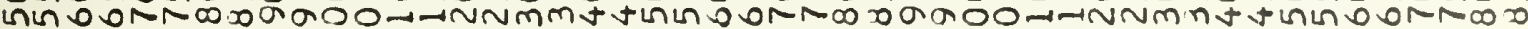
NUN N N N V U

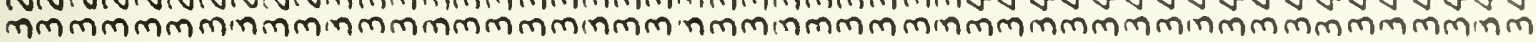

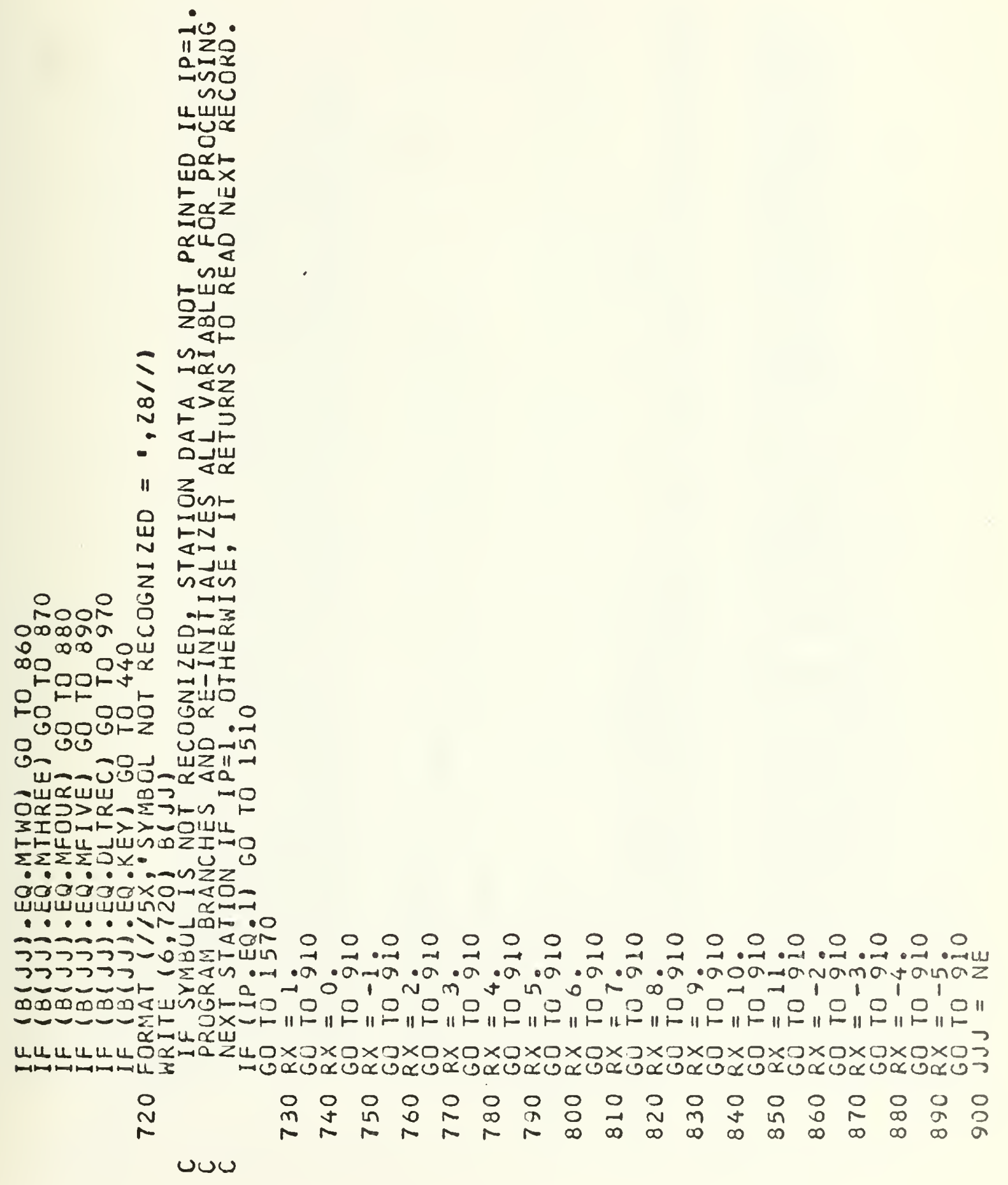





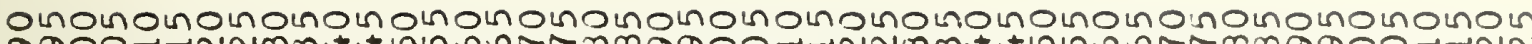

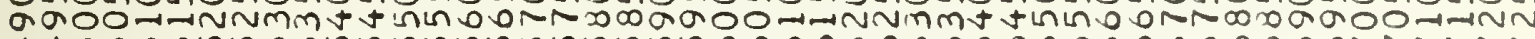

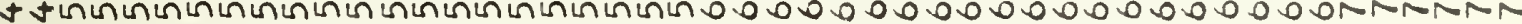
mmmmmm nmmmmm

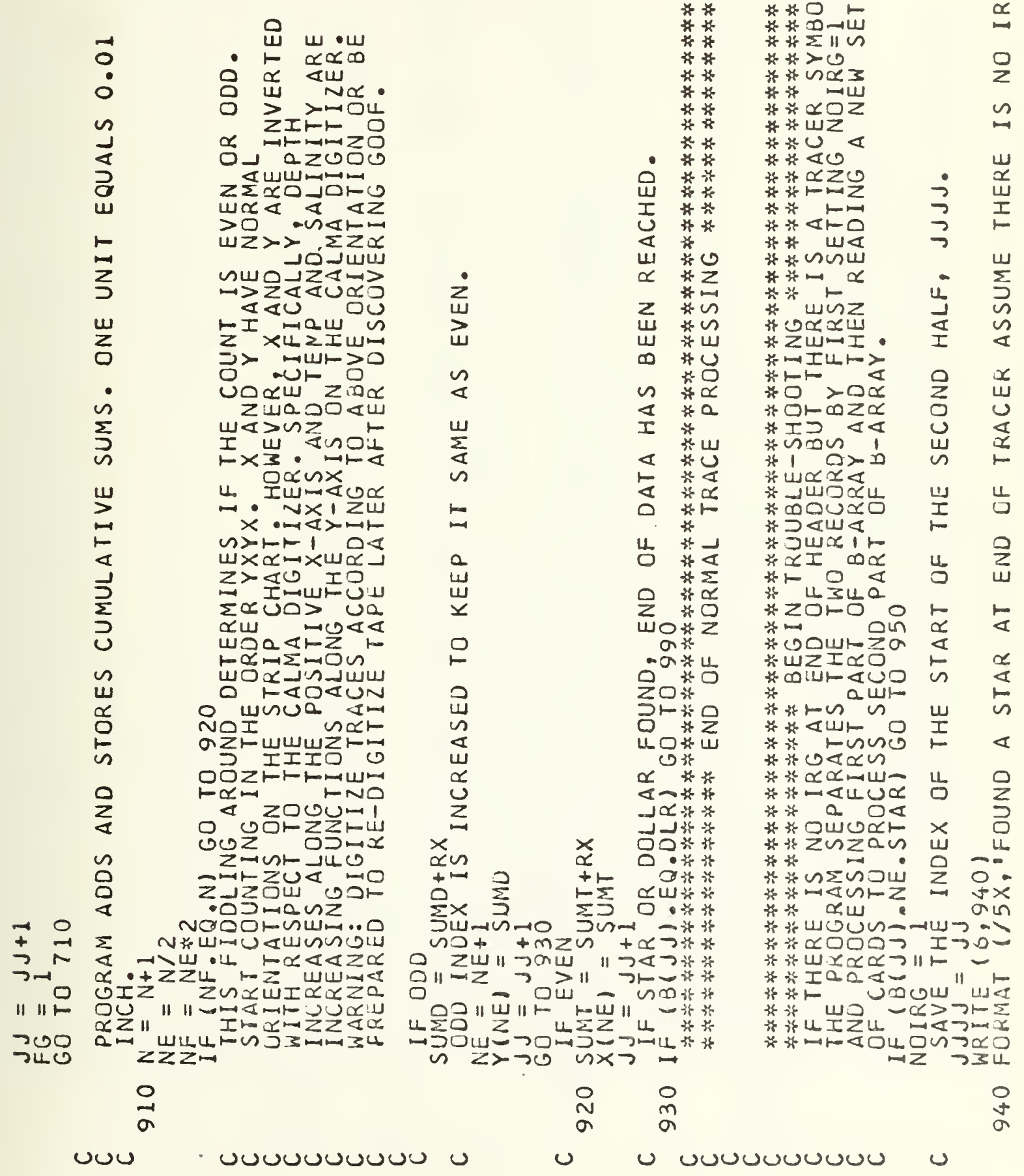





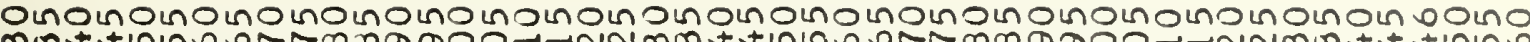

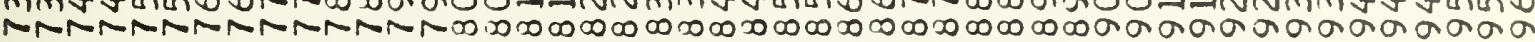

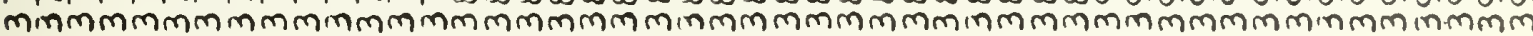

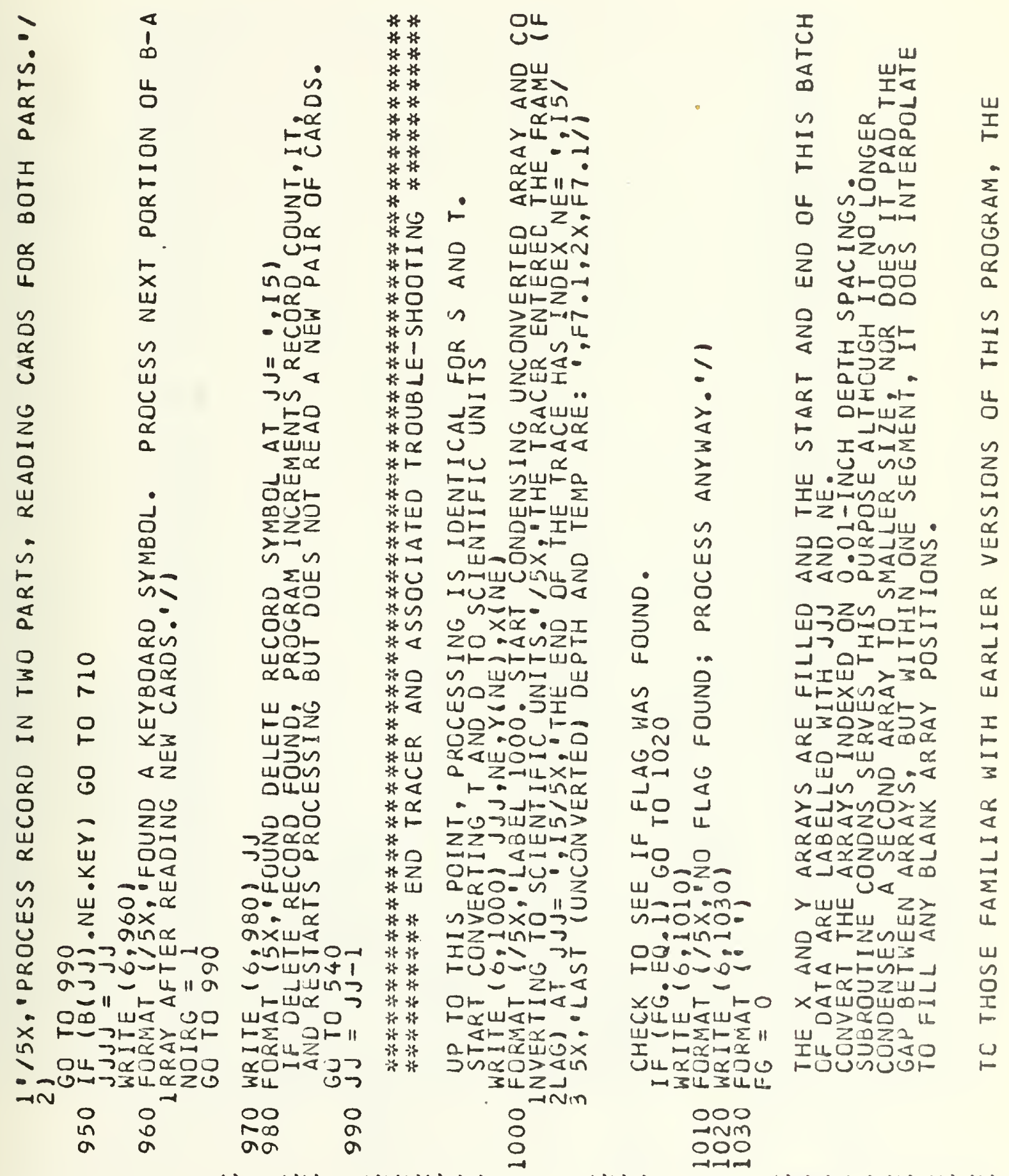





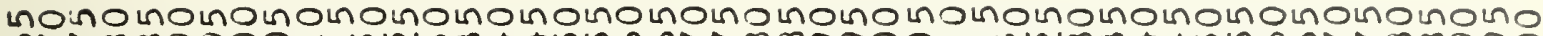

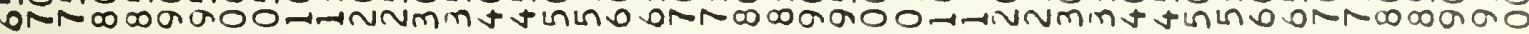

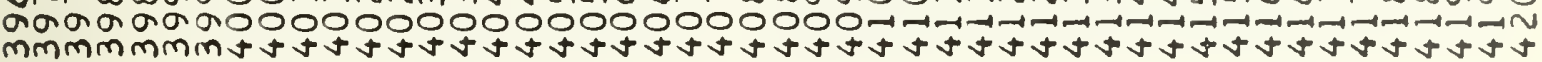

\section{ค $\sum_{<}^{0}$ \\ ш 4 n \\ $2 \operatorname{tin} 2 \cos$

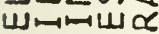 \\ $-\sin 0 x$

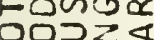 \\ 울민ㄹㄴ \\ 山े ZIO \\ $\propto>-40$ \\ 工只 \\ 뚠ํㄹ \\ $\varangle$ U⿺

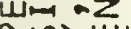

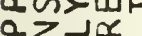

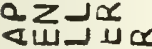 \\ t4 \\ zxum \\ ш山口几 \\ $>u$ \\ แயนเทㅇ

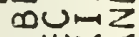 \\ 5 山x< \\ 인요}

$\sin x$

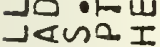

ㅍ⼭ㄴ.

$x+10$

nỡu

แ⿰幺𠃌-

Zथ या

서는

四

० سนเس

๔ᄄ口丩以

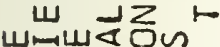

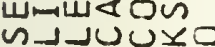

zaan a

山匹心 20

ดแにエณ?

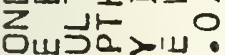

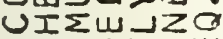

F DF"ய

(1)

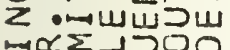

$3<00$ उरo

व_गسயس

inarusm

Jए $\supset 2 \backsim$

Unम

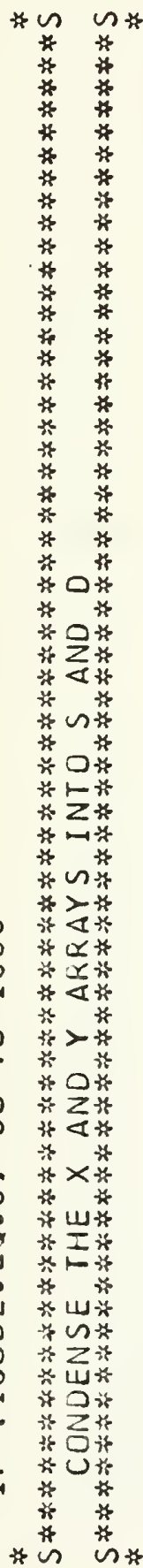

טuuvo

טטטن

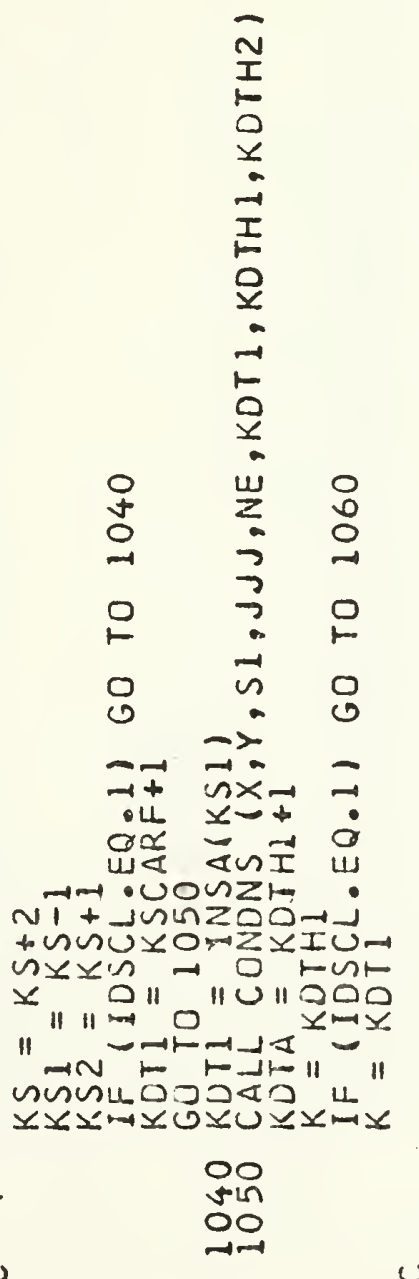

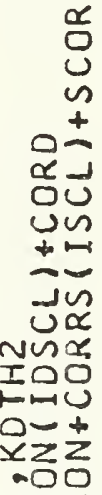

सư

Fov

ำ은

"İニ

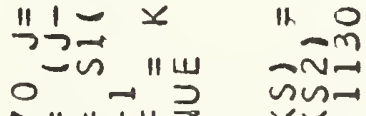

ก" $11+4 \underline{z}$

牙行

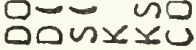

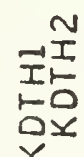

"I

niv-

$\pm \geq 0$

$\varangle<$

जu

ZZO

0
0
0
-1

$\stackrel{0}{\circ}$

माi

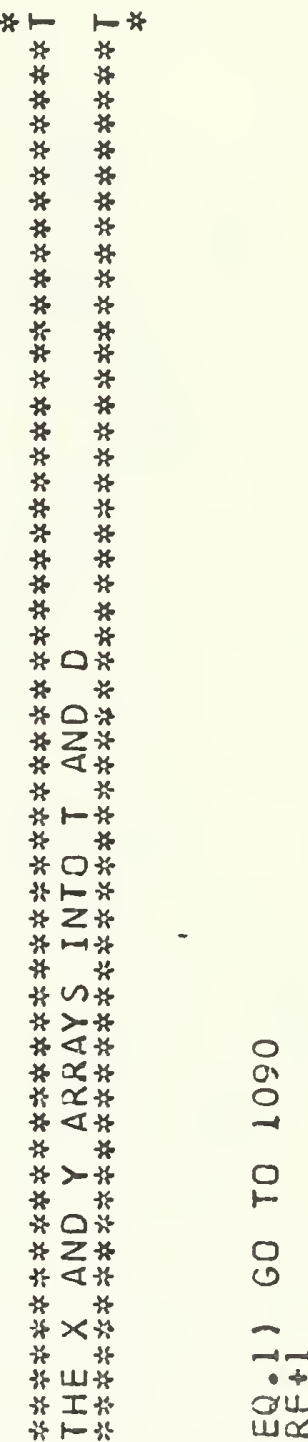

䜤上粠

范山苑

$-1-1<0$

$\mathrm{NI}+\mathrm{NOO}$

*n+ $n+1-6 x-1$

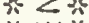

*U⿺辶万

* 0 it

话已铈

"dN

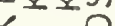

< 01

is $x x y m y 0$

*

* $\quad$ r $\forall 0$

$\infty$ 

unomounoinoinounounomoLnoLnoln

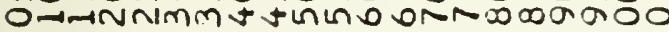

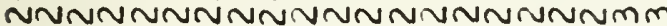

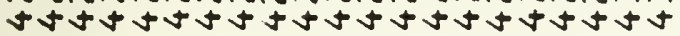

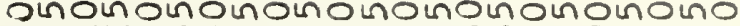

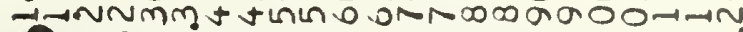
- $10 n m m m m m m m m m m m m m t+t+t$ $a^{7 t a t a t a t a t a t a t a t a t a t a t a t a t}$

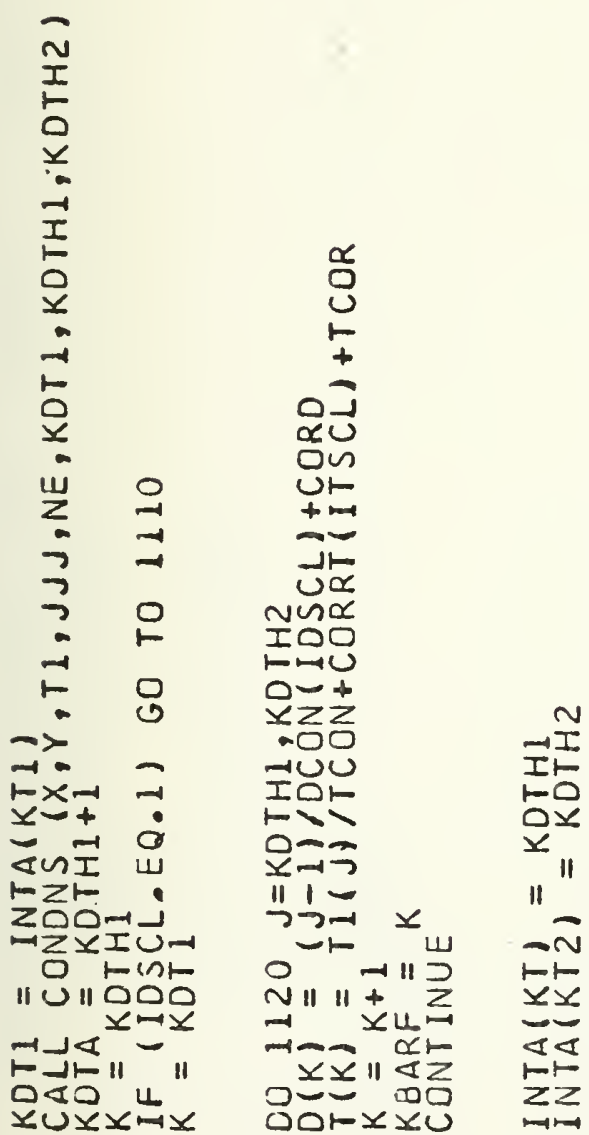

이메

*

* $* 0$ ० 0 $\ddot{*} \ddot{*}$

它

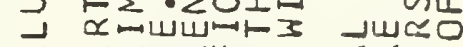

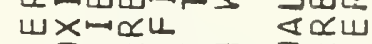

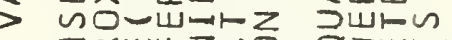

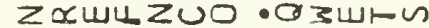
$0 \quad-a>\omega \bigcup \omega$ U $\quad$ U แ⿺辶 $\backsim x<1-\sin \Sigma \alpha$

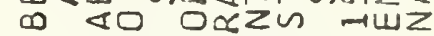

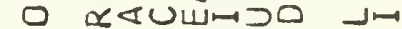

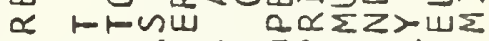

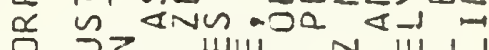

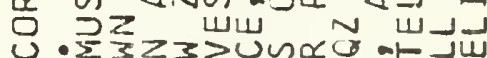

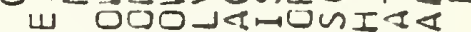

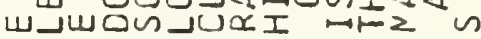

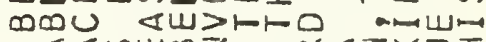

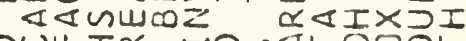

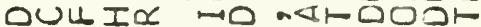
Juar 0 rou

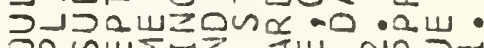

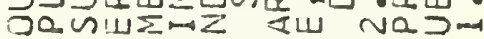
Ia G\&N<UvaW IJ जu $\backsim \rightarrow$ I \&UO \&?

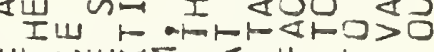

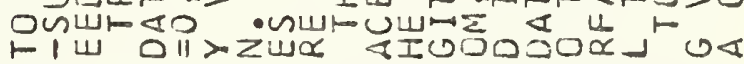

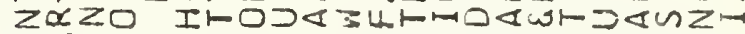
-

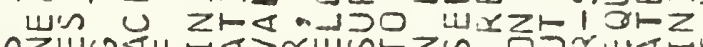

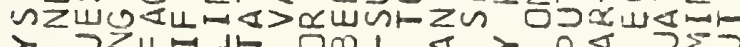

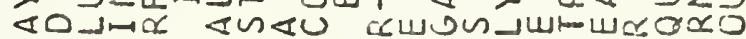

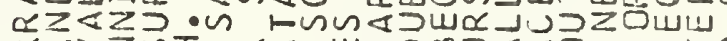

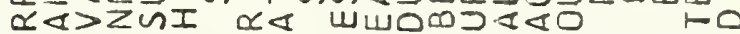

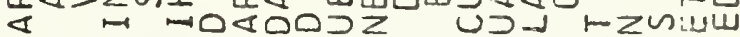

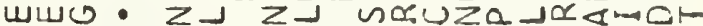
> UUज

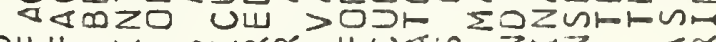
OUL

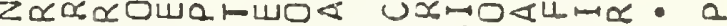

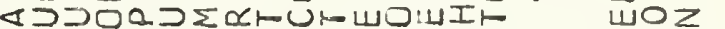
जिए यய

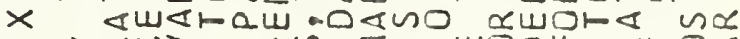

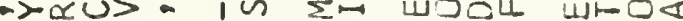

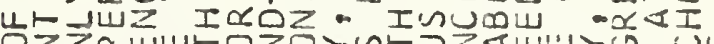

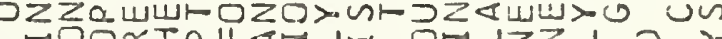

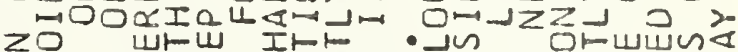

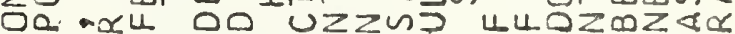

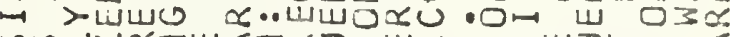
nu-N $\alpha$ Z

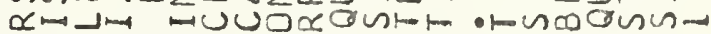

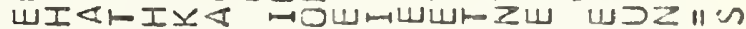

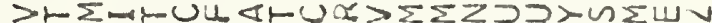

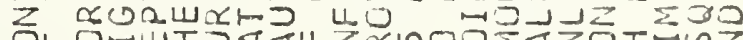

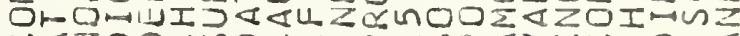

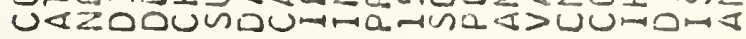



non

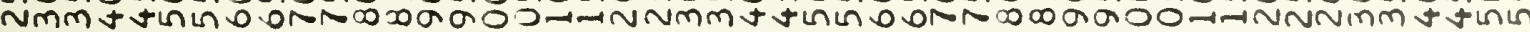

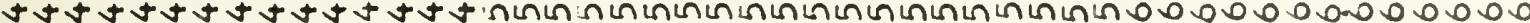

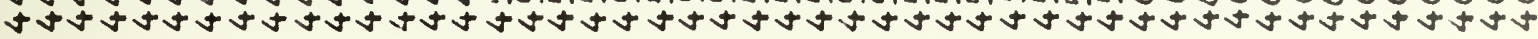

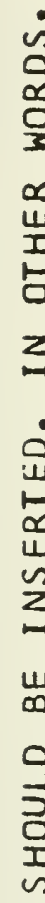

$\sim$

U.

दि

$>$-O

뜬ำ

뜨-

$\rightarrow Z$

In⿺

${ }^{1-}-0$

능요

迥

xயय-AnN

XI:Oトனエエト・

3 Wヘト」N

nú: "

แ山心

जै। ज्ञात

- <u-ruL

$I$

근

Nつフつ

포

ज佂业

*

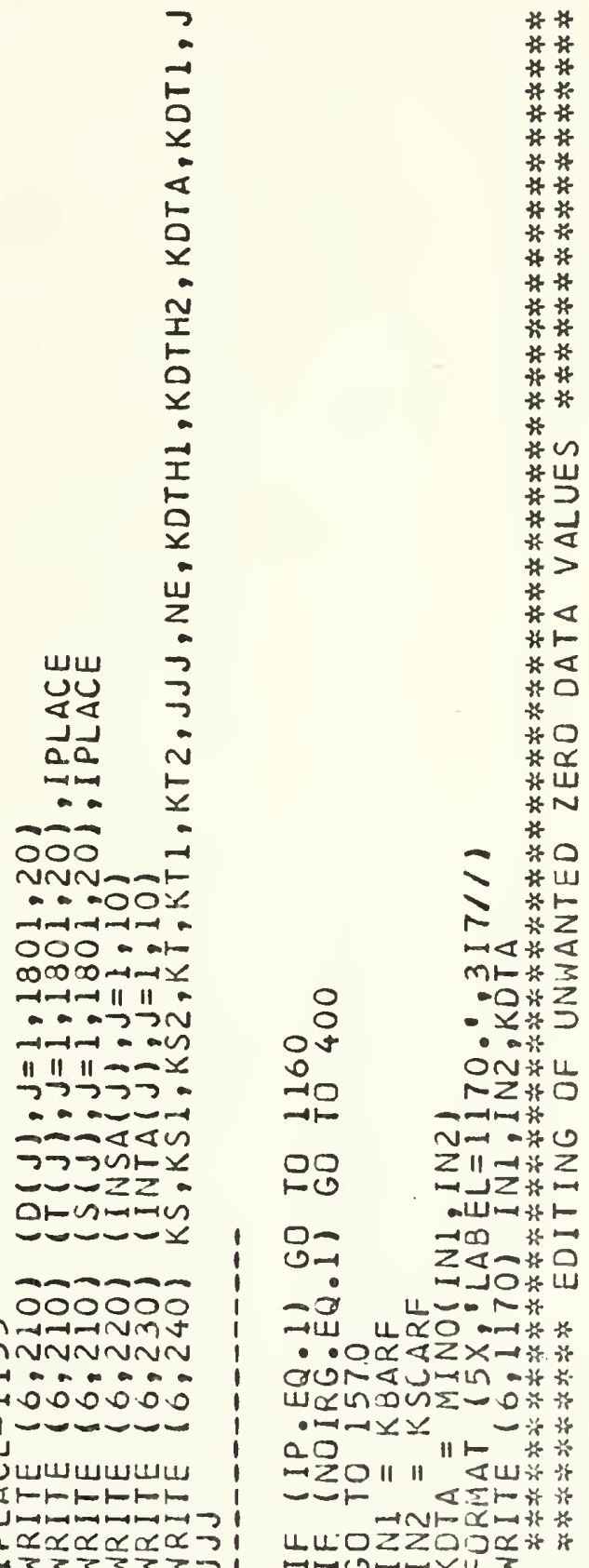

는

ヒヘ 山யメレッ

On $\propto \Vdash \varangle Z I$

ZI $\varangle \varangle \propto \varangle U$ $\vdash \cup x \geq z$

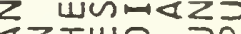

4ZIU口

Uயเつ之o

I J WIx⿺ Jus $z$ Z $>0$ 는 iा $w<\leftarrow$ a nton $⺊<$

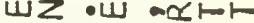
UயOIn⿺𠃊

$\varangle \sum \vdash>2 I 4$ xणD a F ட山之 뜬

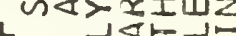
பய. $U \cdot>n z \leftarrow$

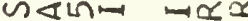

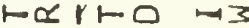
Zr UZau

แ

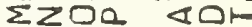
৩UJ凡ト0

แบแयाu $\backsim x$ ง3 व यแ山口 FA IU >U Mr L 10 ○ा " 3 $z \backsim \pm 0$ जU⿺廴

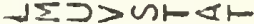
षा० जトト> JW $4 \geq$ แZレ d00

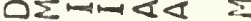
$z 0 \propto Z>z Z m$ বงam

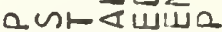

Ia JuNUরU

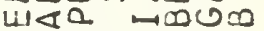
-OHOA

Jzanan WOवसमद Q ZZ $\vdash$ IU⿺ ๓๓யய山ー ๓0 NトI $\propto Z \vdash-$

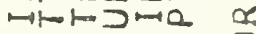
-11 $\vdash 1 \sum \geq 0$

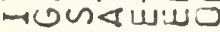
( zะxトルn ๓0ய山аю上ய ดma \& \&

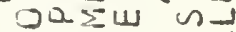
Z゙〉《UI

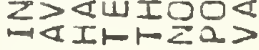

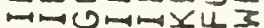

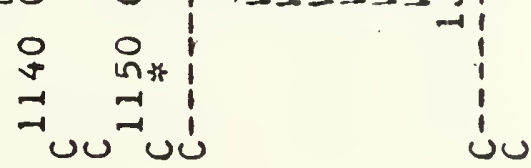

$\stackrel{0}{=}$ 

WNa

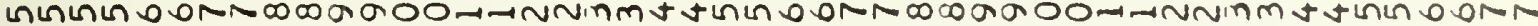
$000000000000-N-N-N-N-N-M-N-N-N \infty 0000000000000000000000$

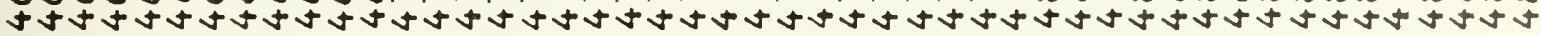

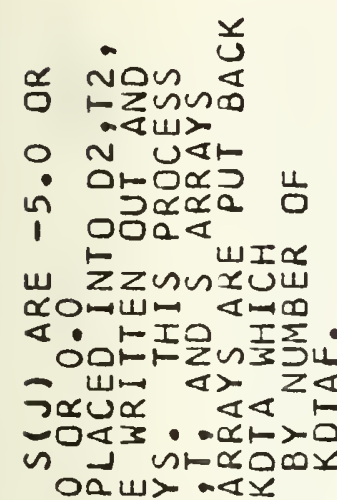

$\alpha$ a

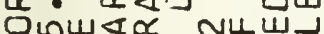

i a वunoum

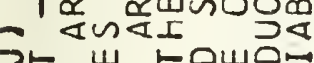

ว上 U

-

a $4<$ a

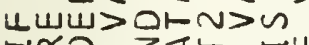

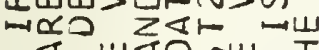

4II\& NIN口

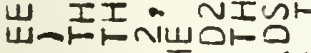

ง

On $-N$ IUU

- oindzトमயu 인 ${ }^{n}$ o o<」uInz

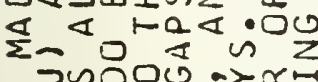
n-W $\vdash$ Q

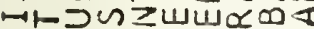

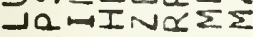

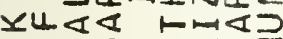

Uே

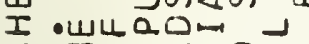

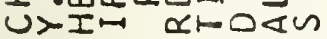
षயम nm>- a

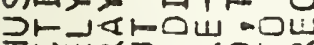

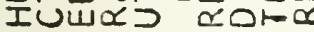
$-\omega>\propto \infty)$

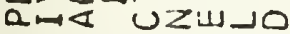
•งเ 0แயIषU ดแUNUUILכட

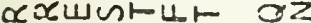
दे खW ०W งоำด - $u \geq 0 Z a z \omega \geq$

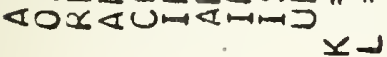
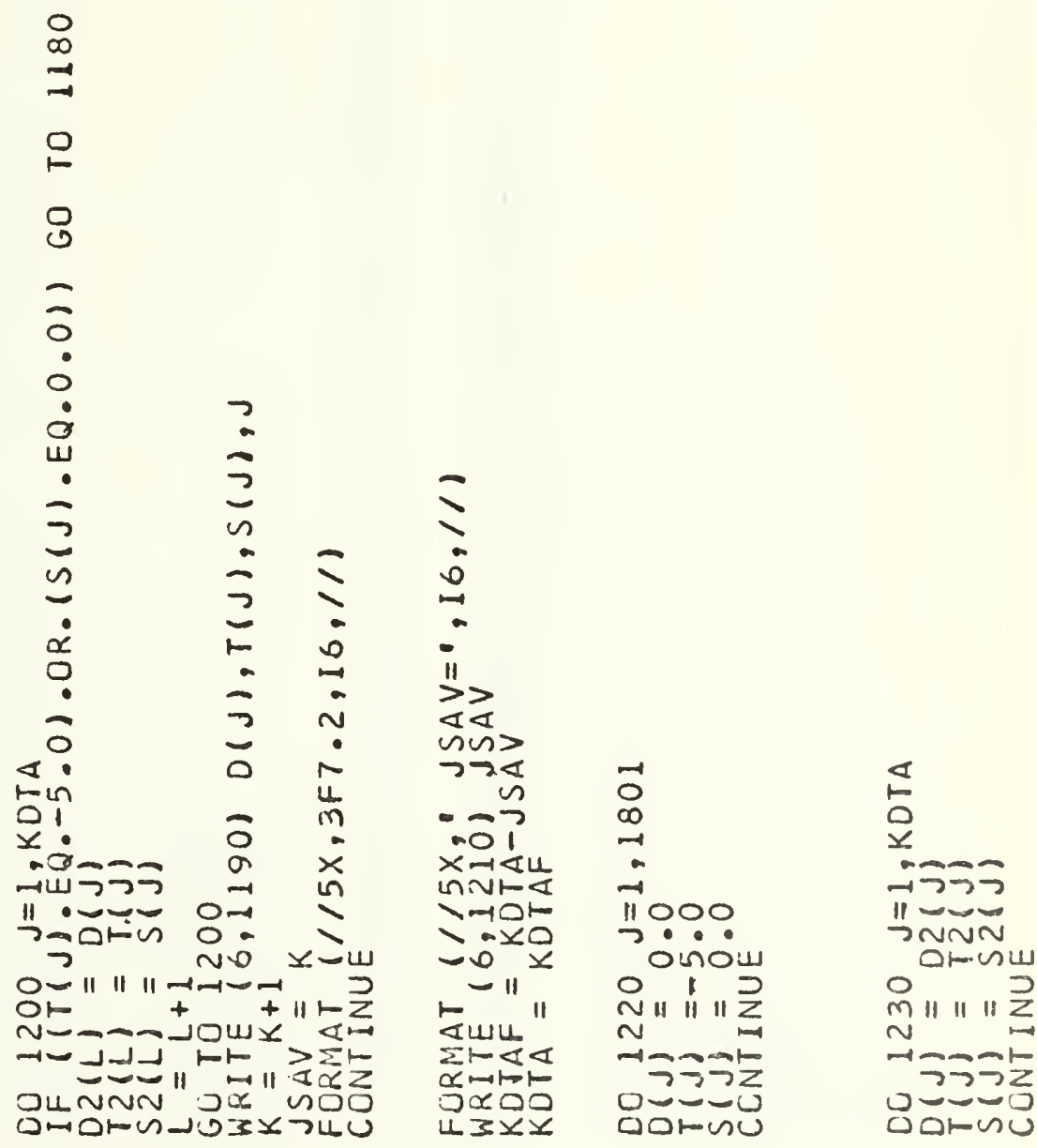

vunumun

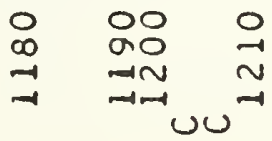

u

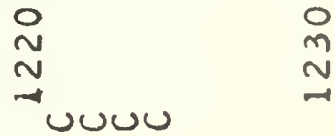





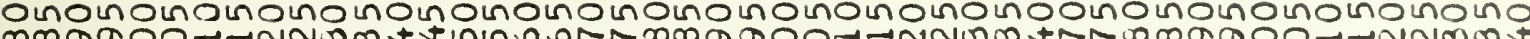

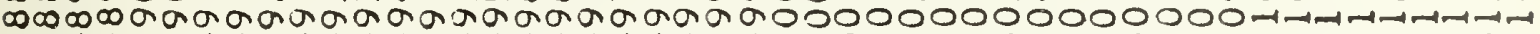

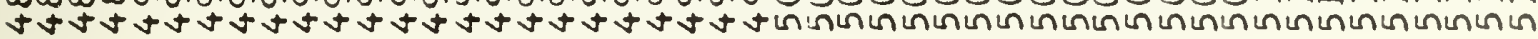

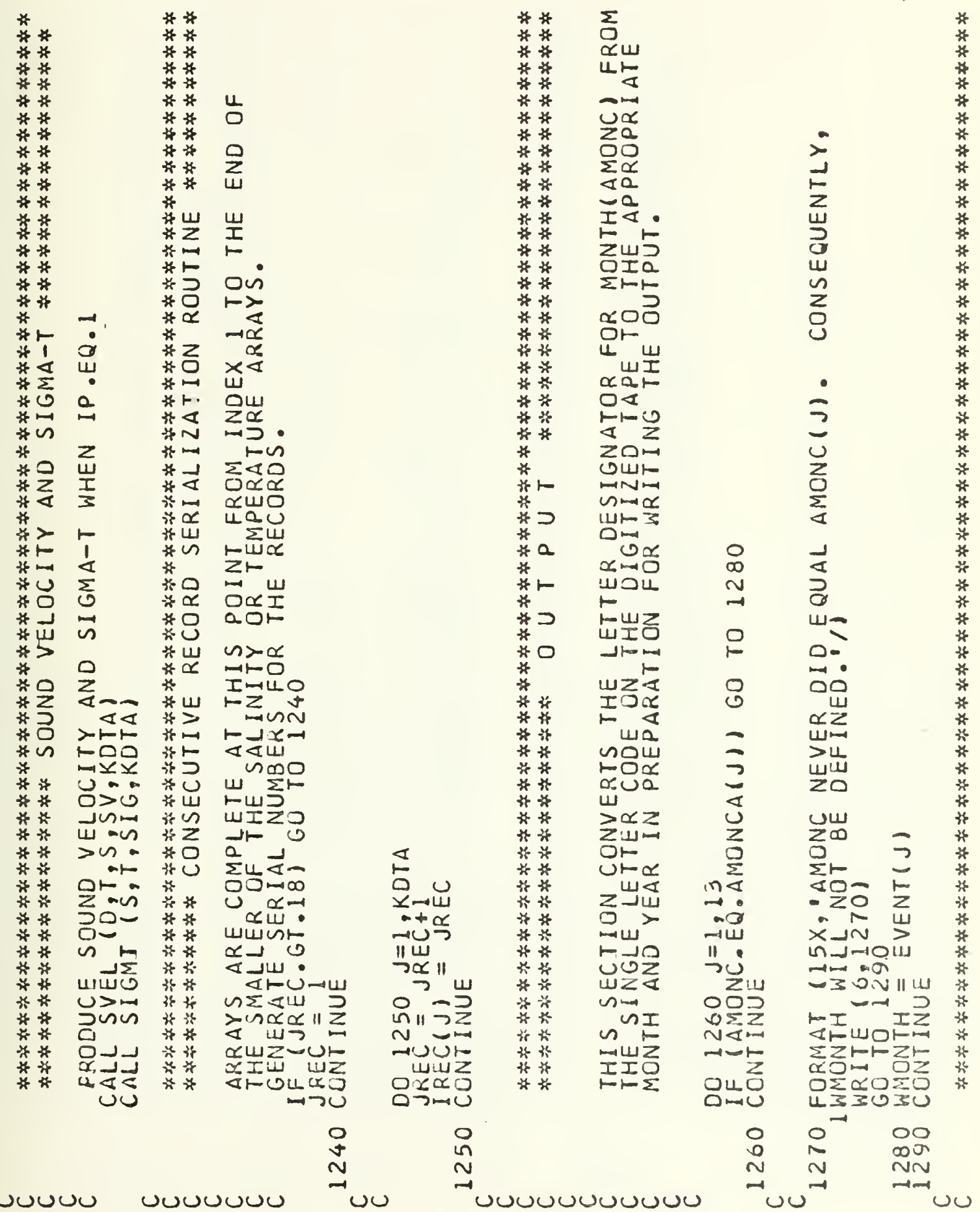



unounounoinounoinoinounounounounounounounounounounounounounoinounounouno

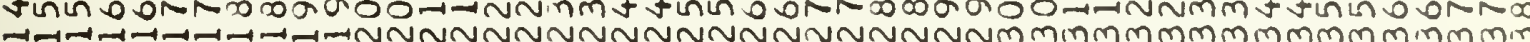

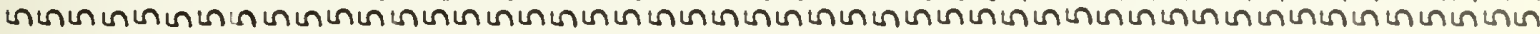

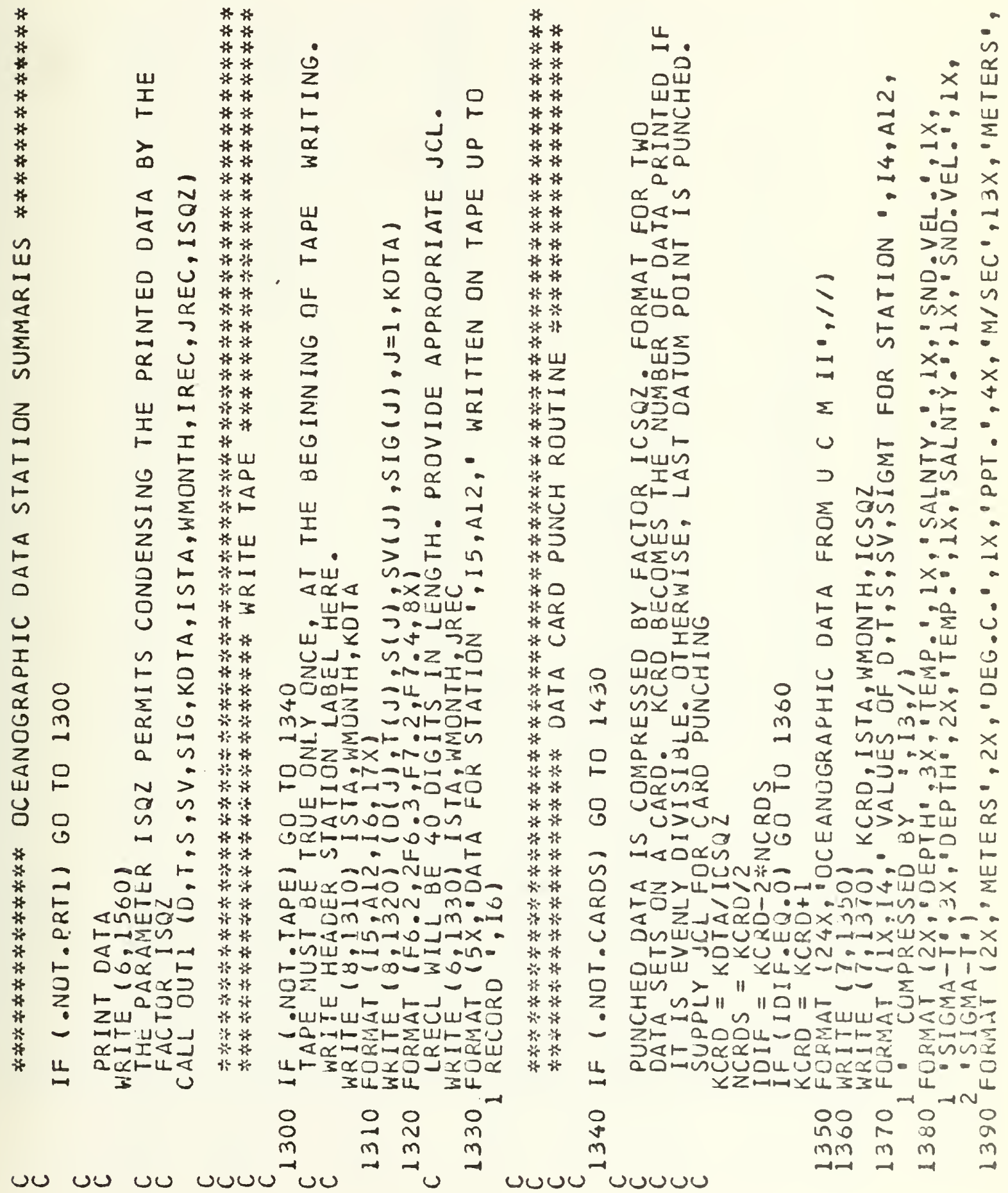





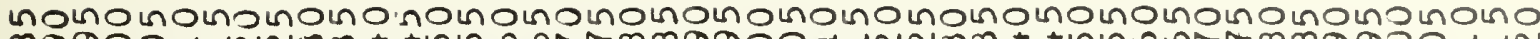

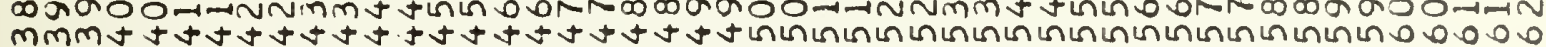

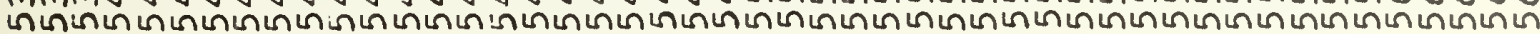

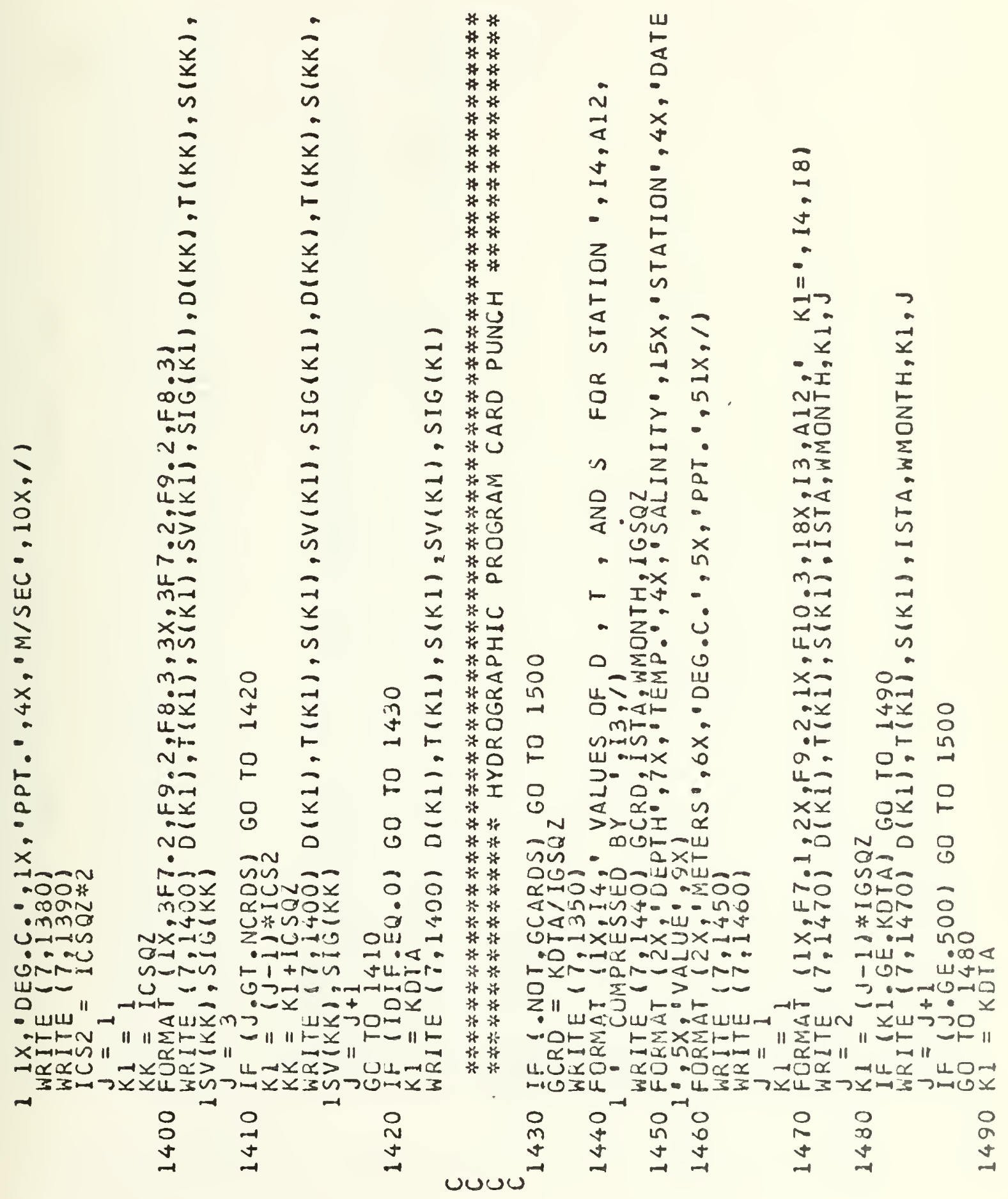





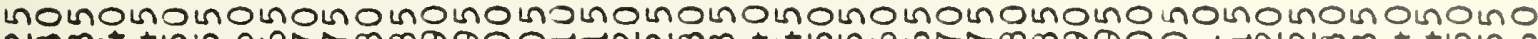

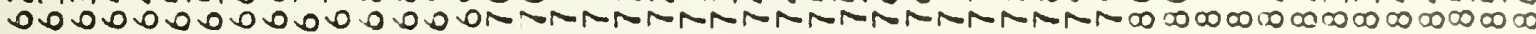

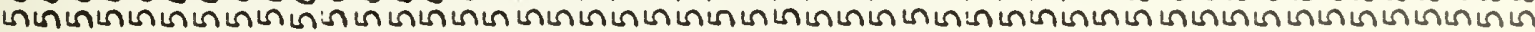

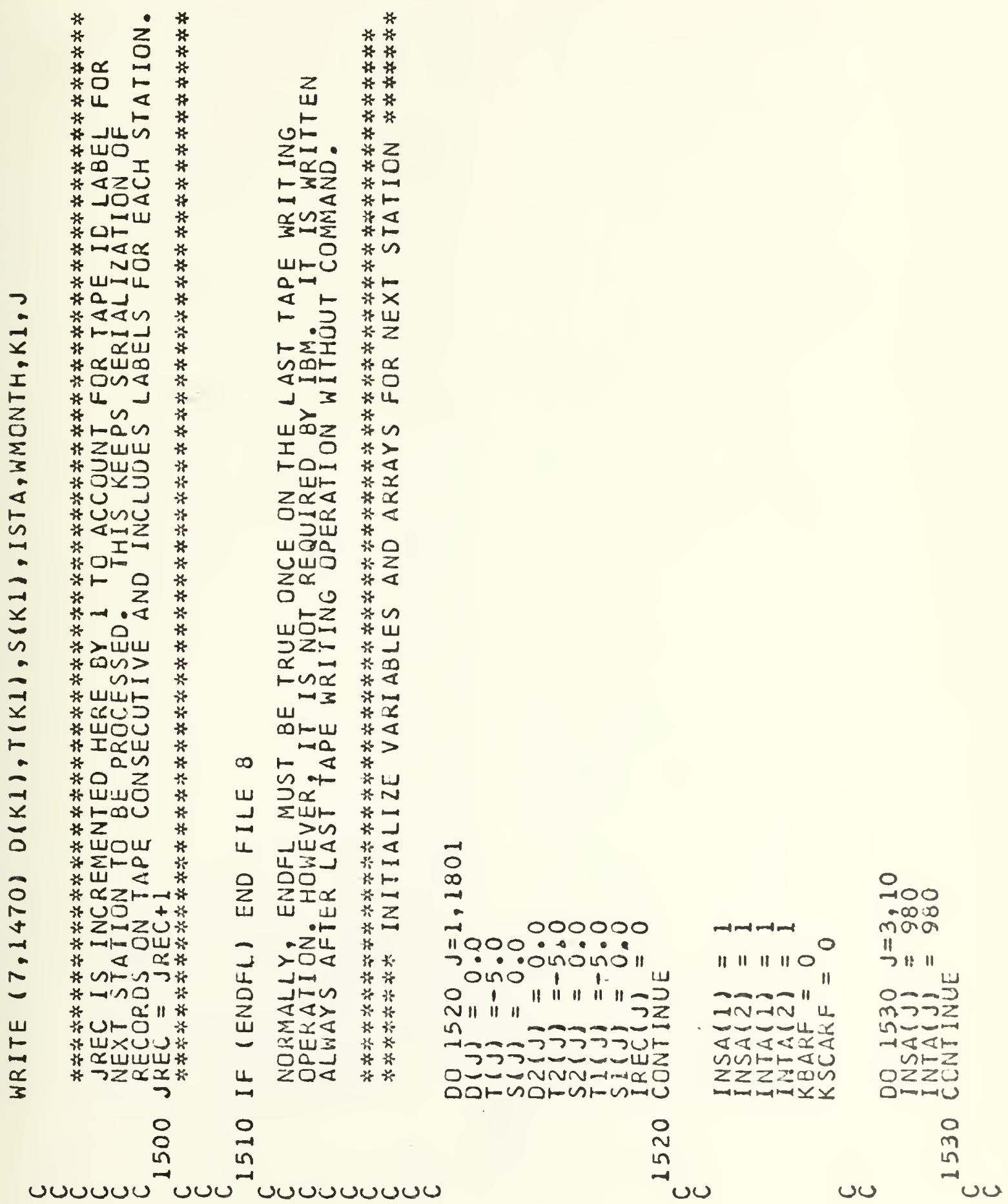




\section{.}




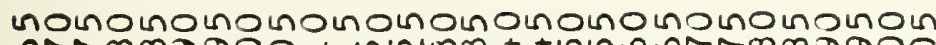
ONA $\infty$ N

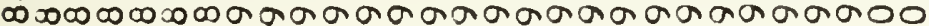

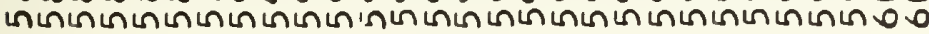

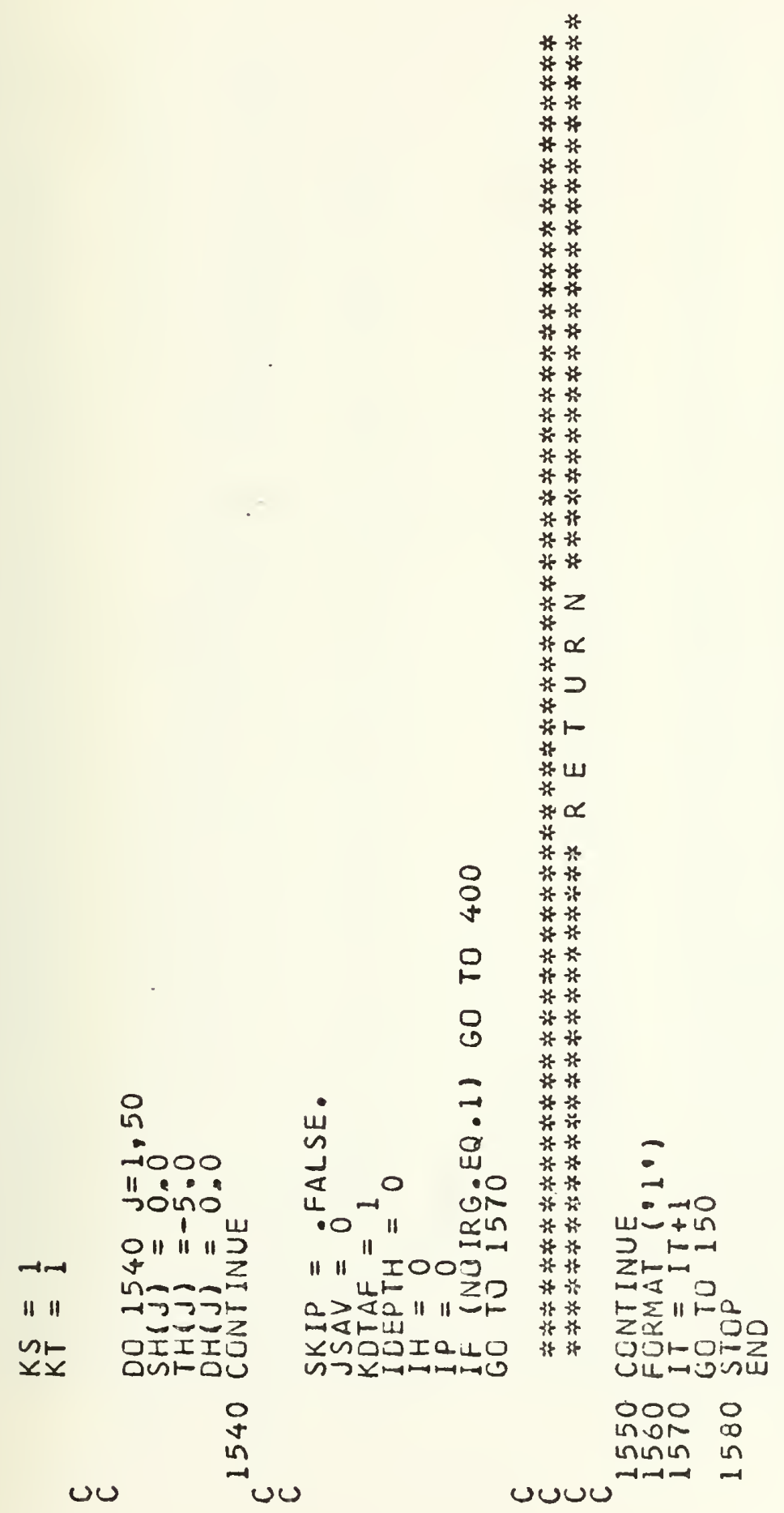





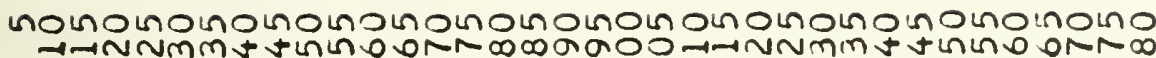

$\rightarrow-1 \rightarrow-1 \rightarrow-1 \rightarrow-1 \rightarrow-1 \rightarrow+1-1$

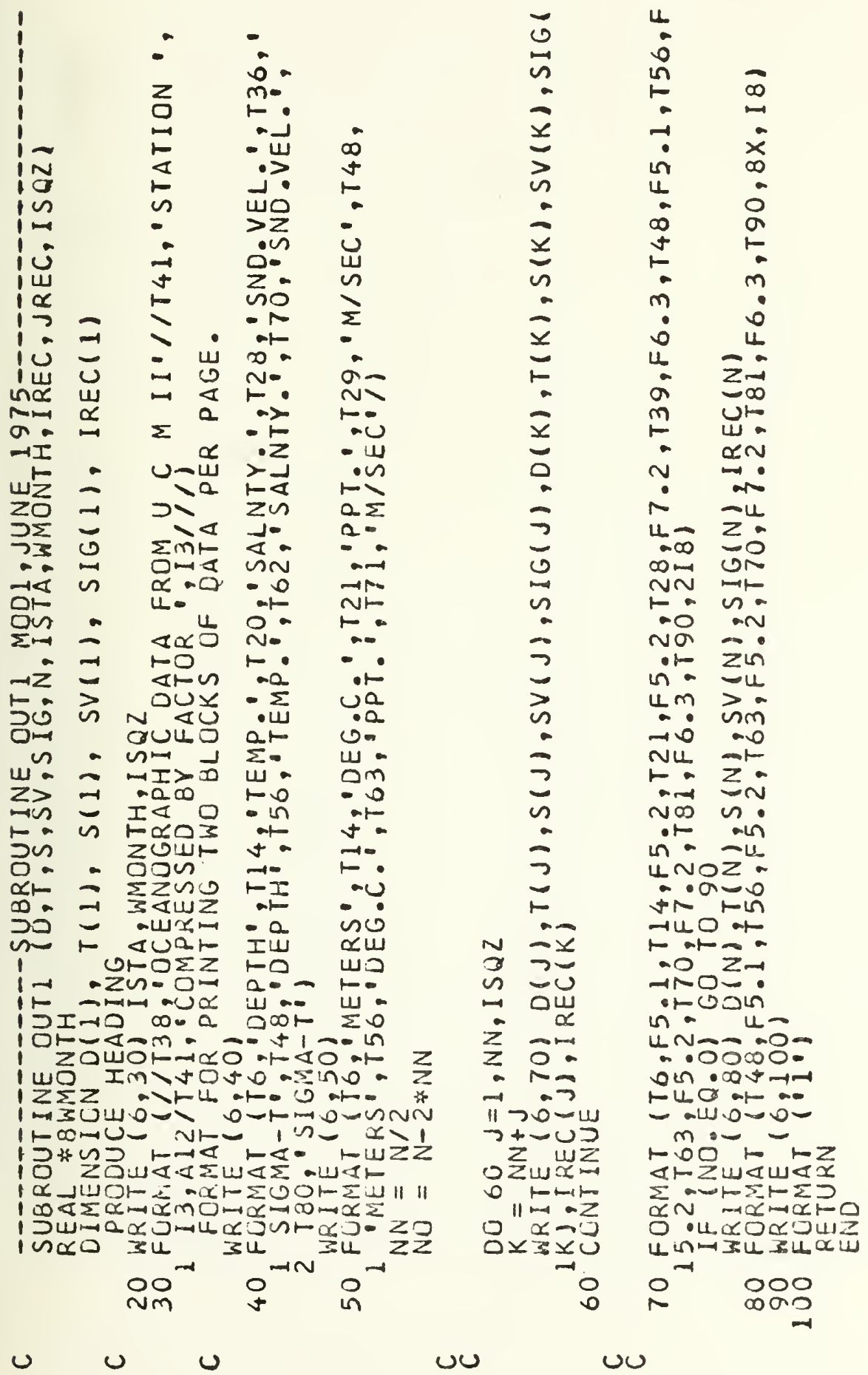



unoinoinoinoin

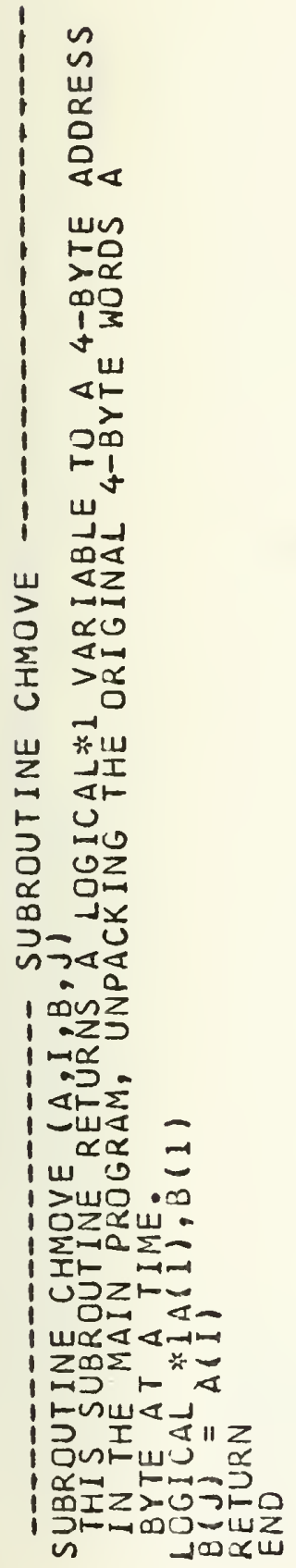

ט ư 



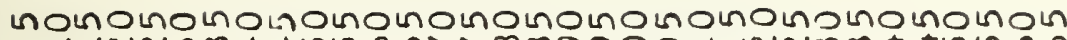

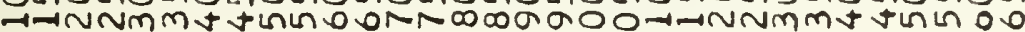

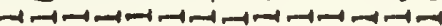

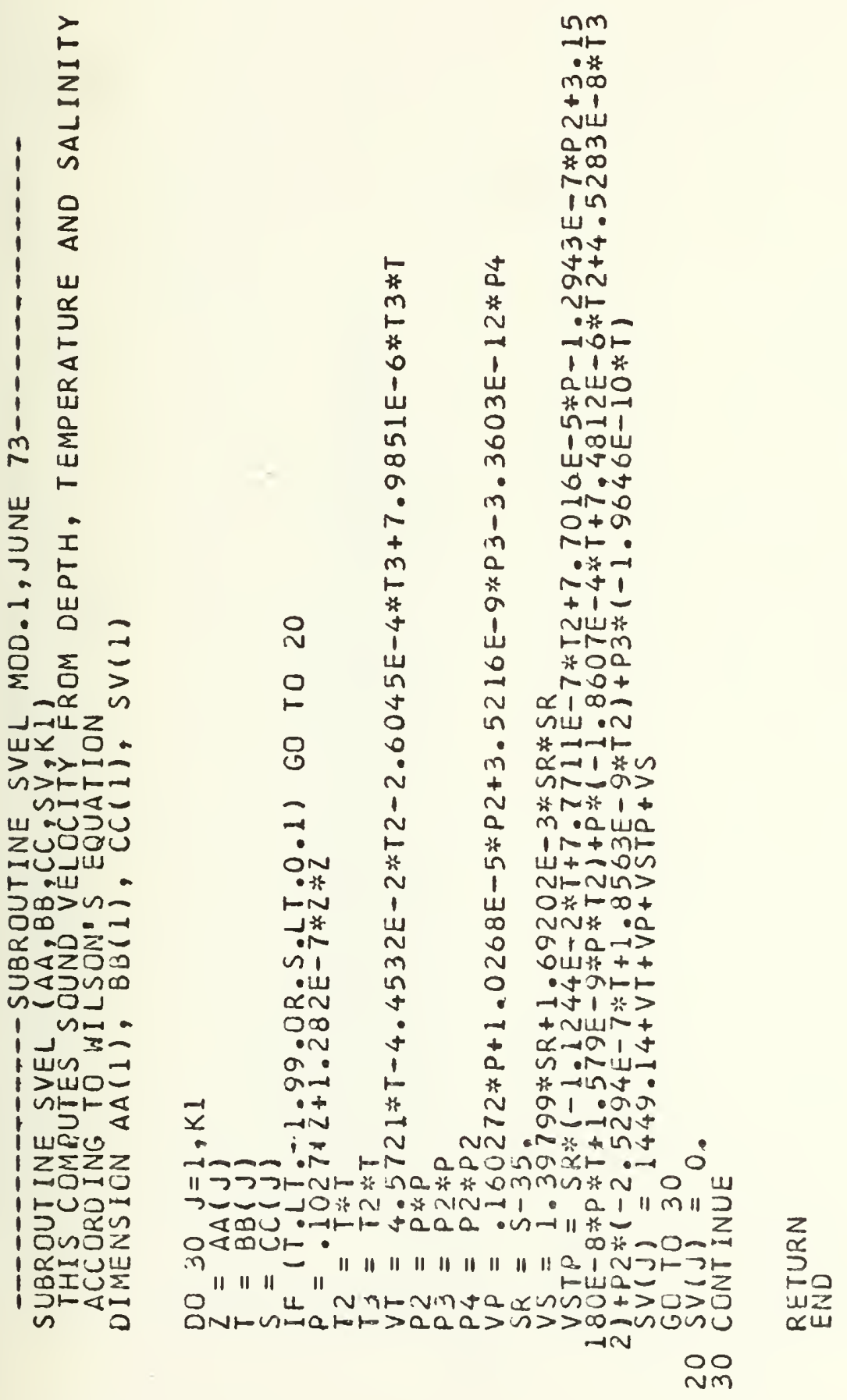

$\circlearrowleft \circlearrowleft \circlearrowleft$

U 



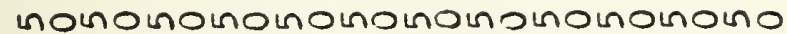

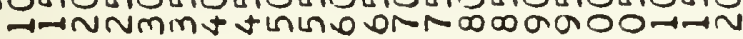
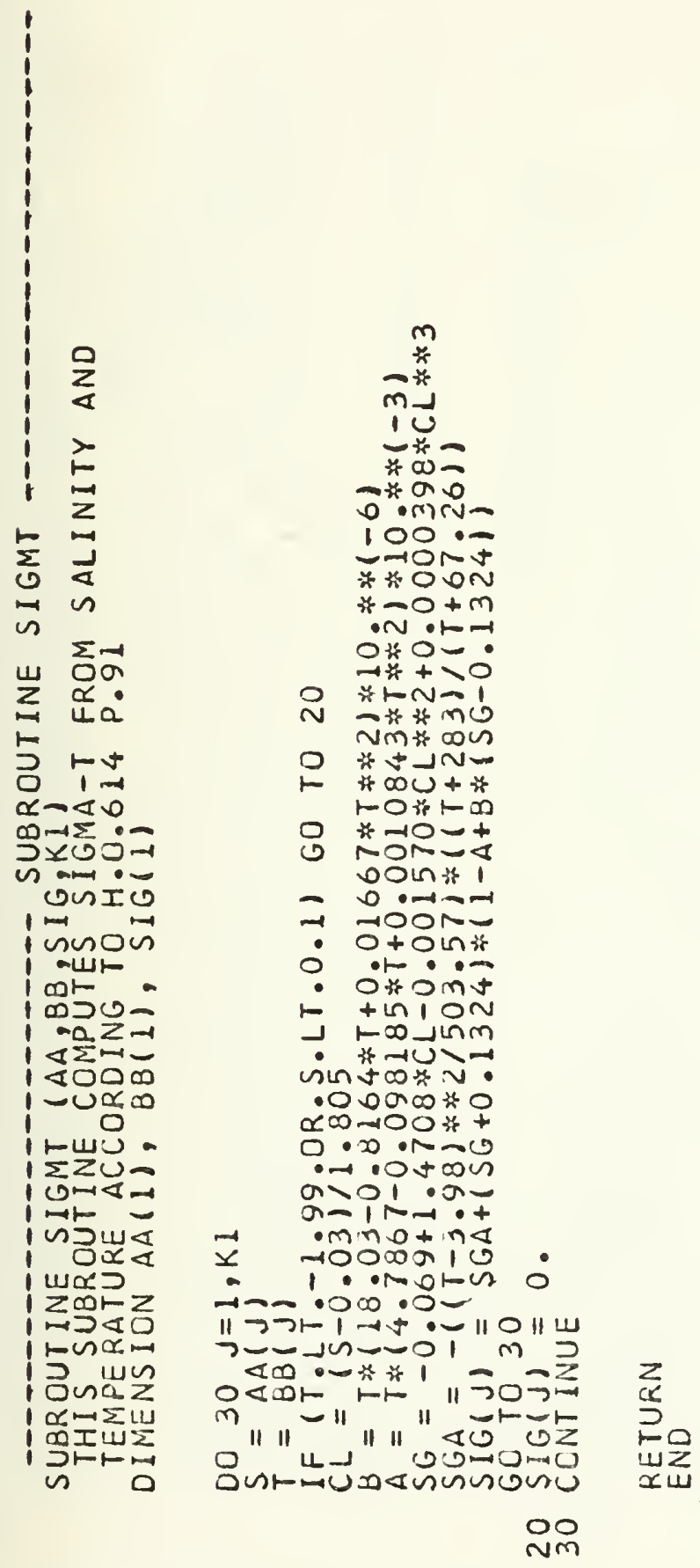

$\omega$ u $u$

u 



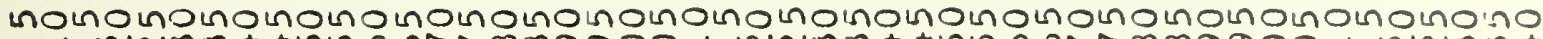

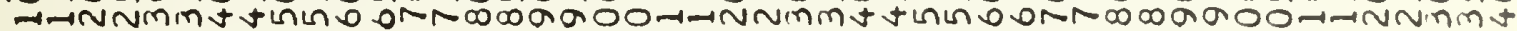

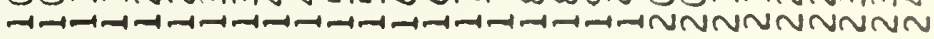

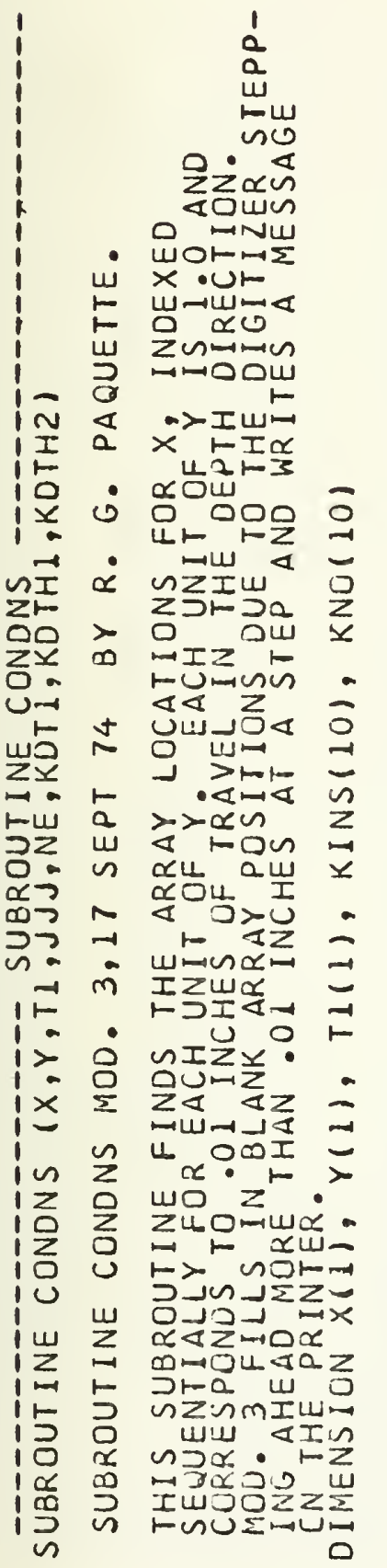

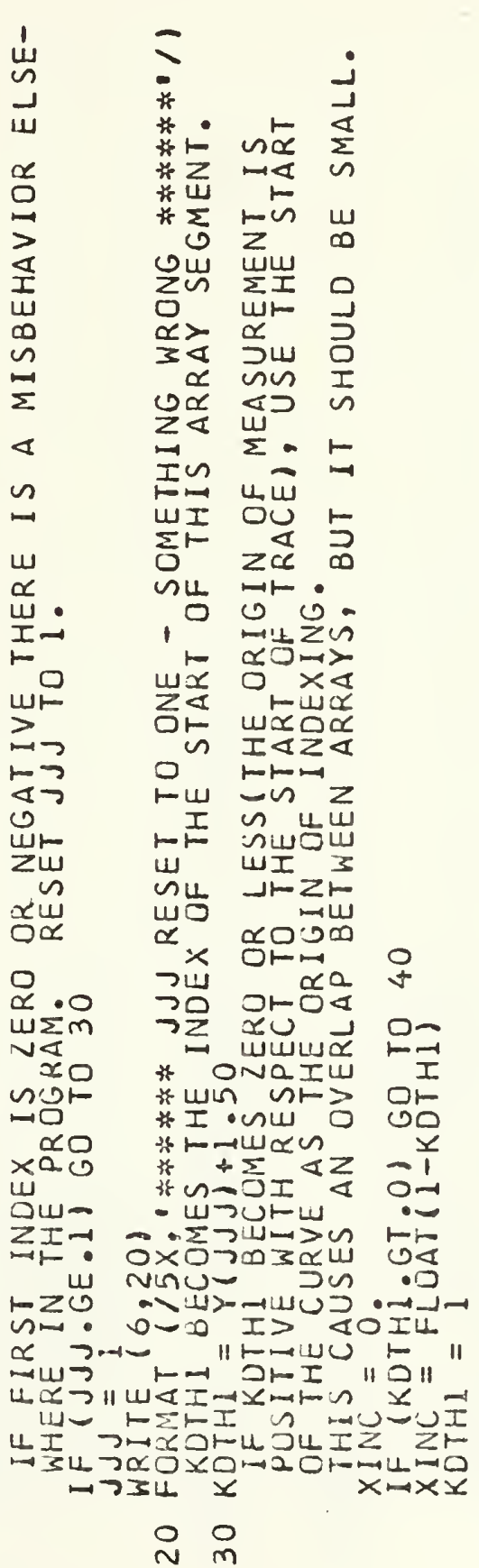

똔

๕

$\ddot{x}$

$\underset{\sim}{\infty} \sum_{\dot{q}}^{0}$

욤

i

ํํำ

$\dot{0}$

$\omega$

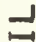

4

\& 2

$\backsim \ddot{\sim}$

z

端隹员

过 $z$

$\sum$ w

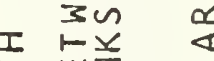

twz

a $\infty<\omega$

㟧

I 我岂

I \&O

$\sum z \stackrel{x}{w}$ 趟

유

4 논

nnz

t-Z<ய

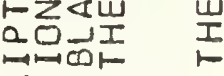

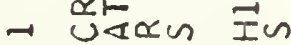

h-nuor Fn

ᄀI

ว山د

ᄀZn I00

$11=>0$ U $11=2$

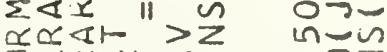

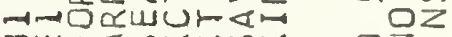

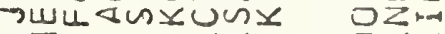

$0 x x$

요 in 



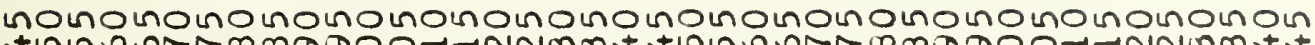

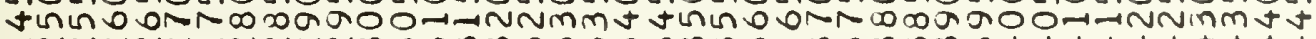
NNNNNNNN N N Nmmm

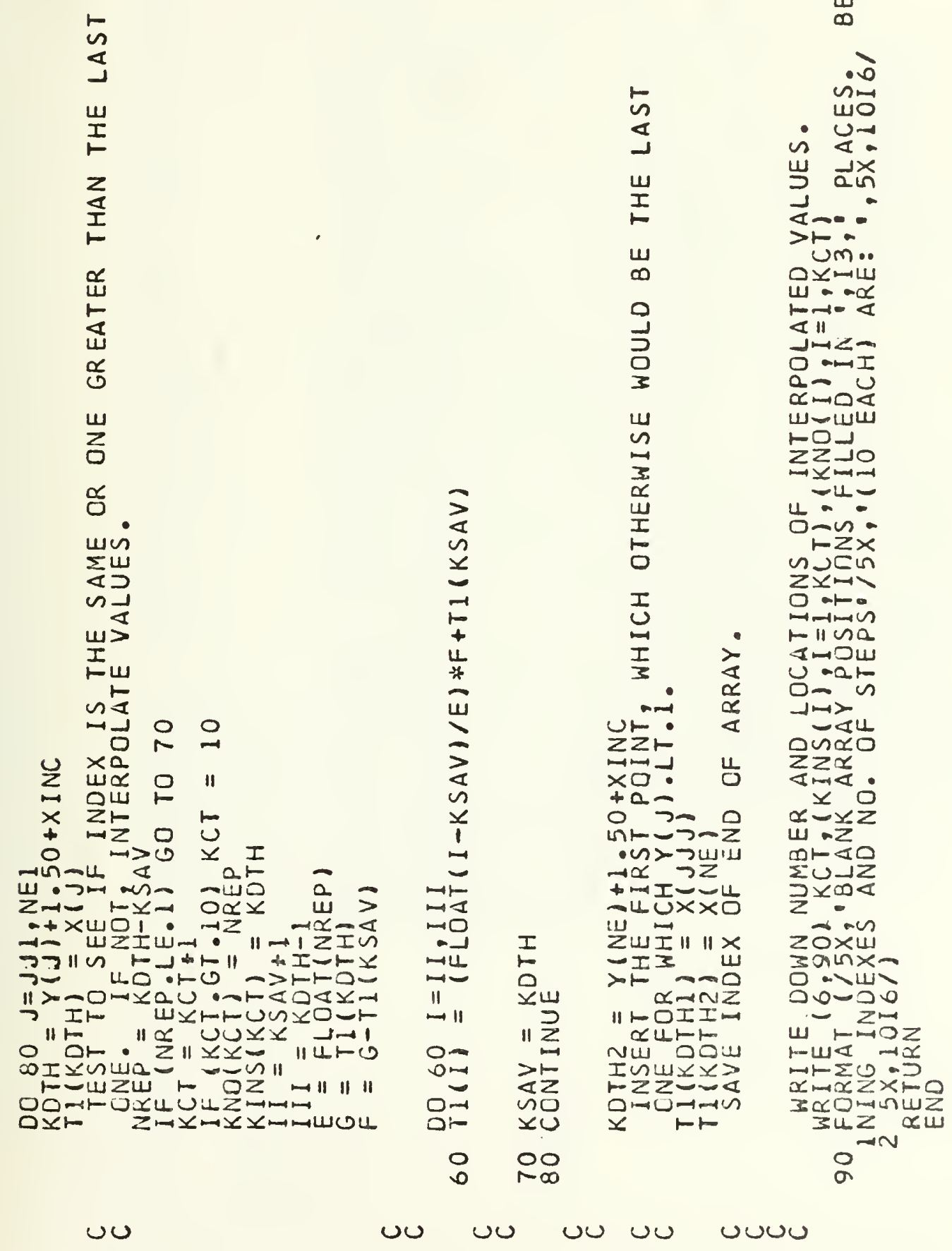





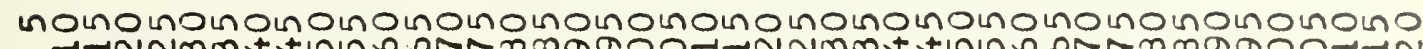

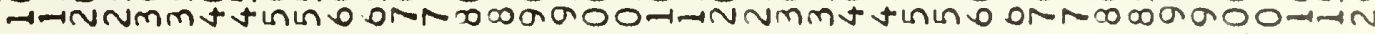

$\rightarrow-1-1-1-1-1-4-1-1-1-1-1-10$ N N

us

$x$

$\mapsto$

$\square$

2

u

a.

a

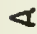

2

-I a wmu

UF $Z \sum$ I 0

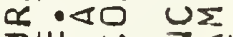

DU $\circlearrowleft$ Zun

tul

$\alpha 1<00$

แ $-\frac{\alpha}{\alpha}-\alpha$ z

10000

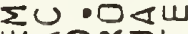

U⿺辶⿻

$4 \alpha$ a 0 Z

$\mapsto>\quad \square=Z$

$01 \alpha \cdot>\alpha+u$

แलแया แ

$N>00 \quad-3$

$\mapsto \propto \omega<Z \backsim u r$

-u $\alpha \mapsto r-\omega$

$\rightarrow N \alpha H \rightarrow 0 \infty$

$0 \backsim 0$ I $2 z$

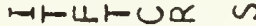

$0 \rightarrow \infty<\omega \cdot 0$

जดхயนเ

ง

woxwxoar

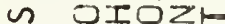

ज小r-U<Do

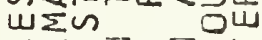

ง ZwZ

$0<00 \propto 0 \omega<$

xงz כma 3

a $<1-1-<$

$<z<<1 \infty$

Quxro $\backsim$

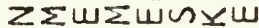

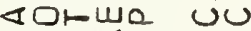

$\alpha D x \leq \omega<0$

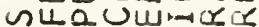

$\propto \tilde{u}$

$-\sum N$

$0<1$

$\propto \Sigma$

wn

$\infty \leq-$

$\sum 3 \sum$

$\infty \quad \exists I \alpha$

C JIN

$\rightarrow \quad \cdots 0$

UI $\alpha$

- 30

$c$

\begin{tabular}{l}
0 \\
$u$ \\
0 \\
$\vdots$ \\
$\vdots$ \\
\hline \\
0 \\
$\dot{\alpha}$ \\
$\dot{\alpha}$
\end{tabular}

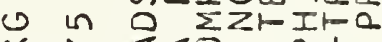

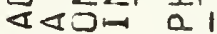

யru 0 \&

$x \leq I \sum x x^{t}$ i

$\Sigma$ - 10 000

II

वr

vat awar-

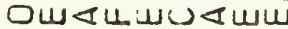

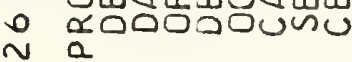

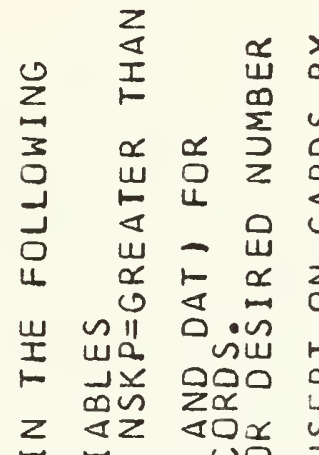

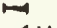

$\propto u$

$\sum \sum$

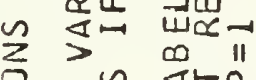

$\sim$ It口

Z๐

$4<0$

$z u$

$\supset$ n

42

w $\propto u$

$\sim \propto 0$

I

I $\mathscr{x}$

num

$0.4 \quad Z$

स०口

טw

งทีZ

IUt:-sus

षU⿺𠃊u

这

$\sum<\sum$

品 $\backsim \bar{z}$

U

$4 N x$

w

$\alpha<$

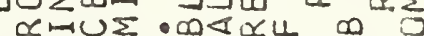

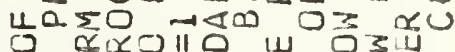
ग40

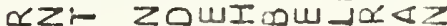

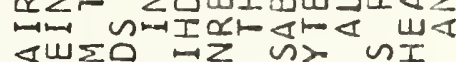

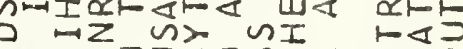

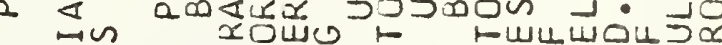

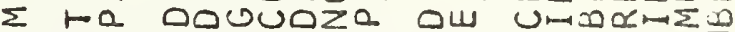

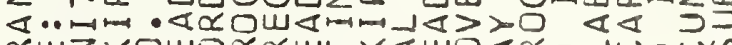

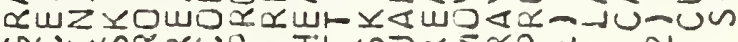

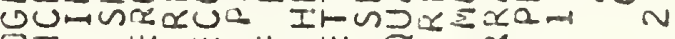
$0 Z$

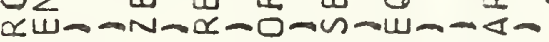

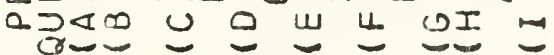
Iw
U 



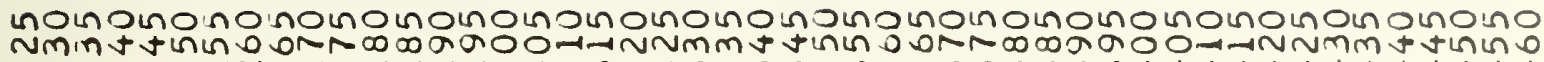

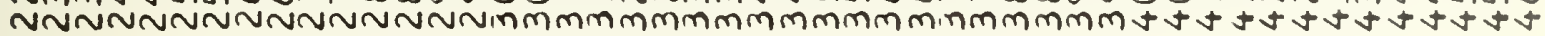

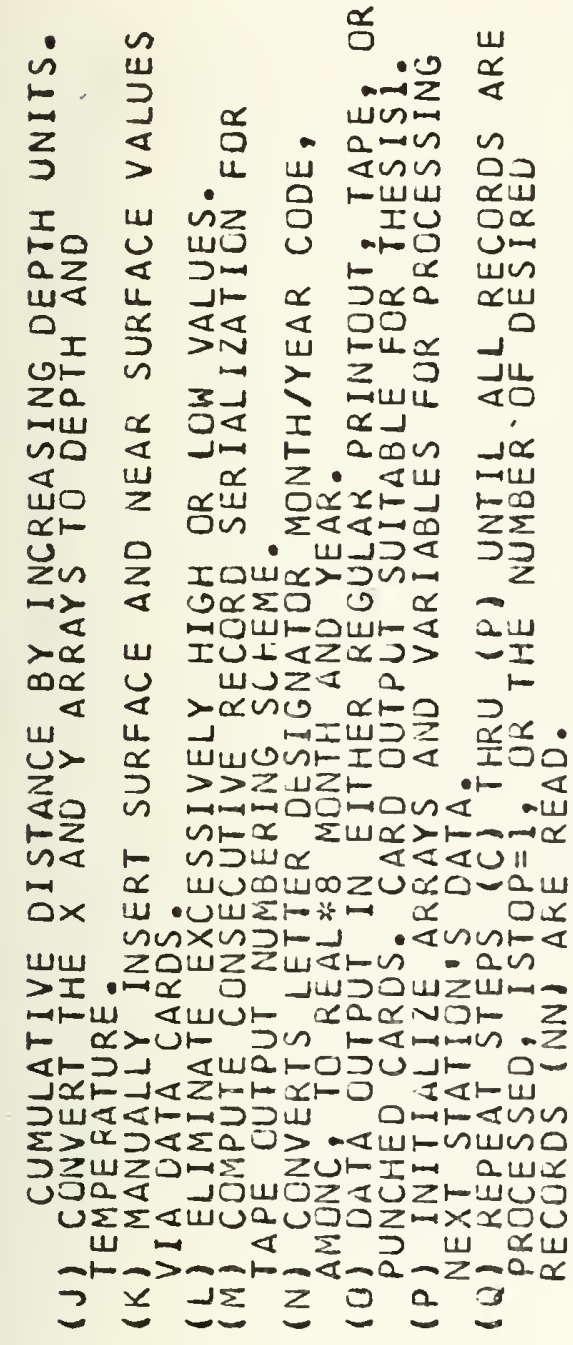

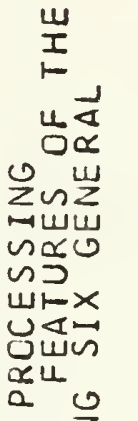

$\cdot z$

는

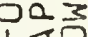

4. 40

$\sum_{x \rightarrow J}$

a Uu

(1)

Oجس

årI

0.15

N

w o

$\lambda \sum$ 㟧

$1-\infty z$

《i⿺

ก

$\propto<0$

writ

$>\& 1$

on

خد

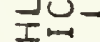

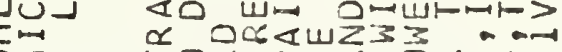

эгш..

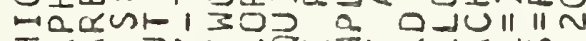

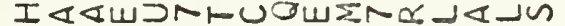

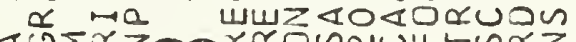

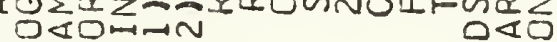

$\sim 200$

- प्रणW-

யढ口 4

suax<

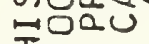

I

$u$
a
w
$u$
$u$

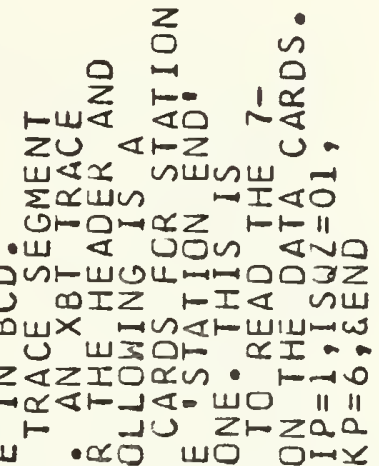

2004 \&I > an-

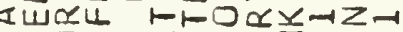

-QO

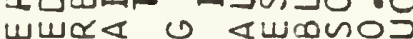

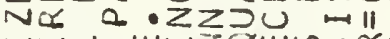

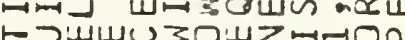

แ⿻コ一า

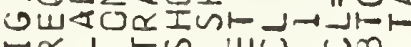

nan

nanuxu $\sum$ -

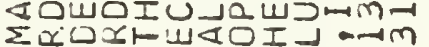

Jel 1 O

3 T

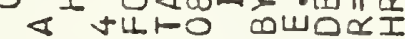

$x+x^{2}$ <nI aODF

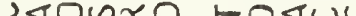
$\omega$ $>\quad>$

告

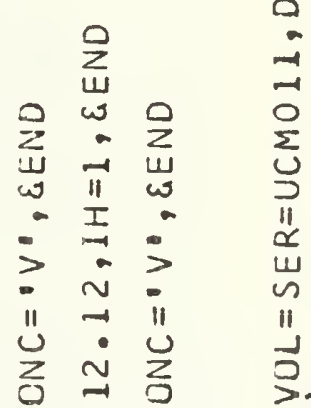

$\sum_{i=1}$

뜨

111

OII

+1 0

$N \propto \alpha$

$\| 1+\infty$

$1+\infty$

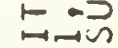

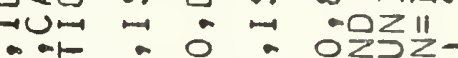

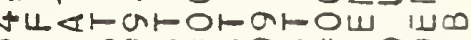
O\| -U山nX\|XIX\|xIZ0 $\| a I F I F O$

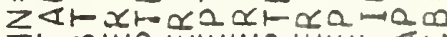

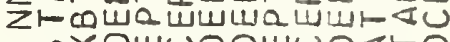
-xоயบ00யบ $0<1-0$

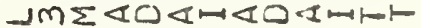
वै Uumar w口x जा FNDIFI I ZN马I $\begin{array}{llllll}\bigcup_{\omega} & \varangle & \varangle & \mathbb{1} & \mathbb{1} & 0 \\ \omega & 0 & 0 & 0 & 0\end{array}$

$$
\begin{aligned}
& \sum \\
& 0 \\
& 0
\end{aligned}
$$





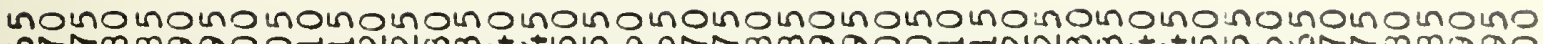

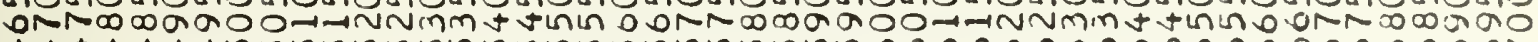

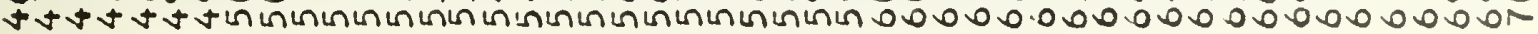

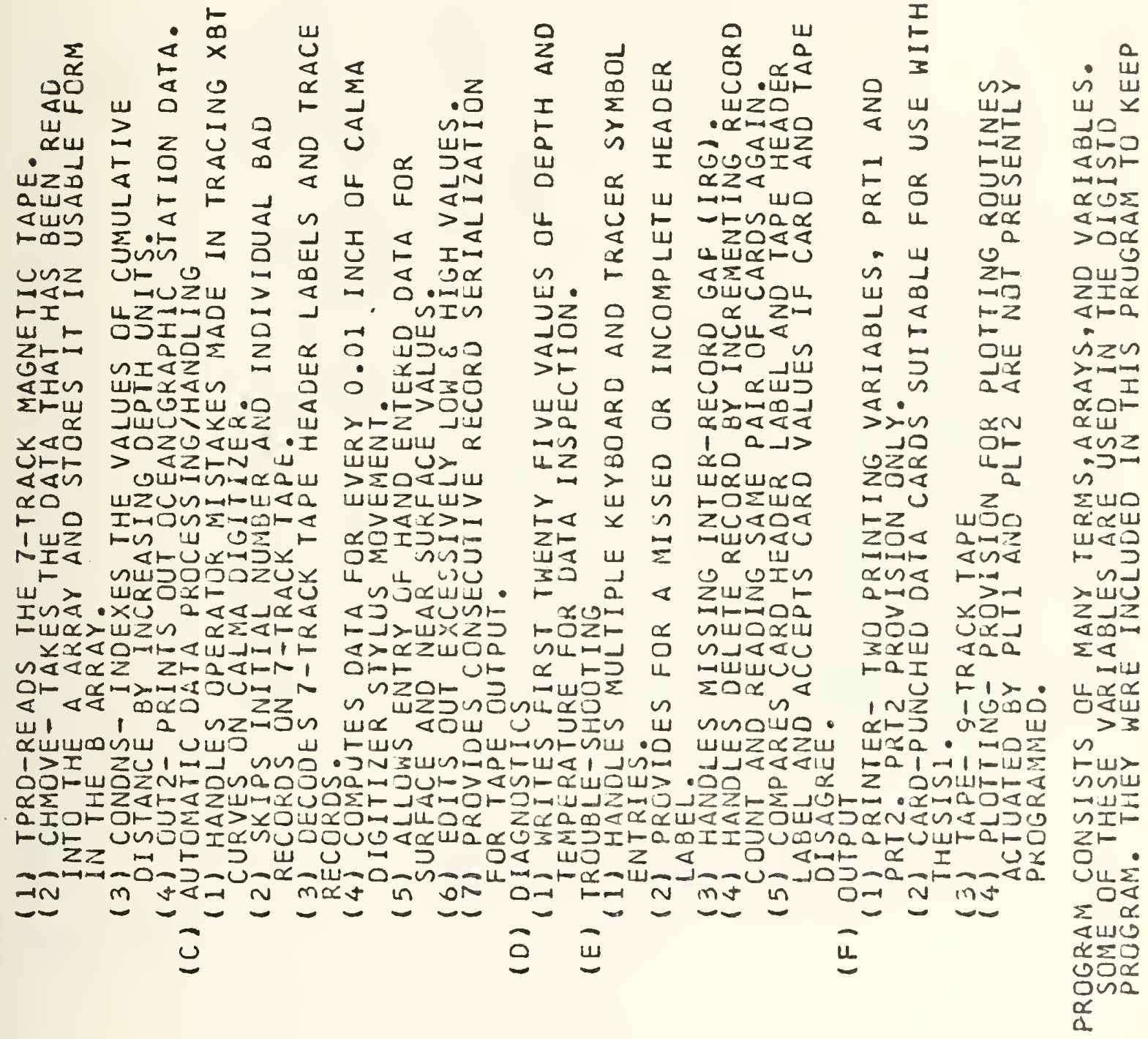

$\sum_{\substack{1 \\ \hline}}^{n}$ 



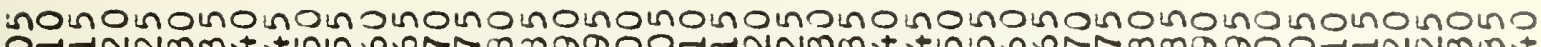

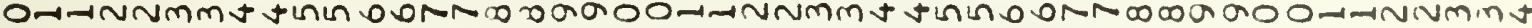

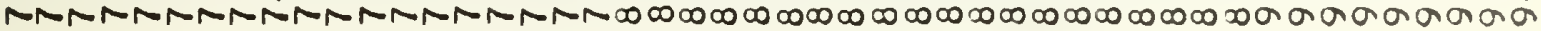

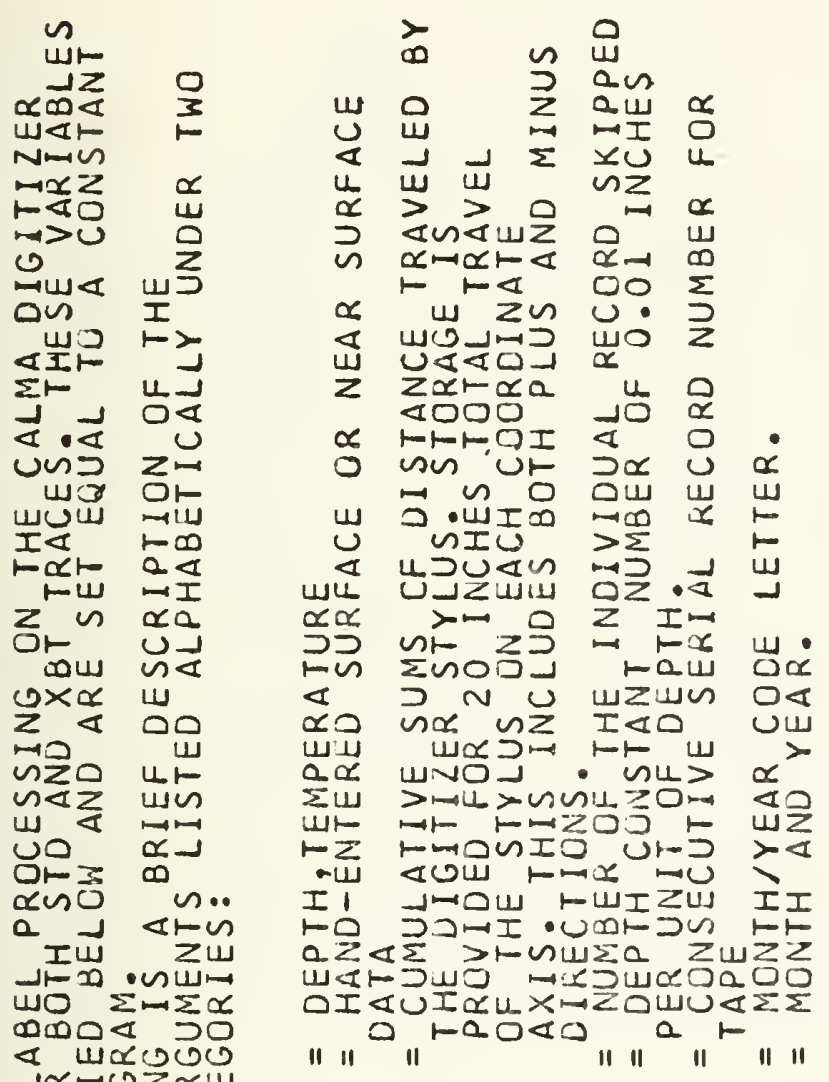

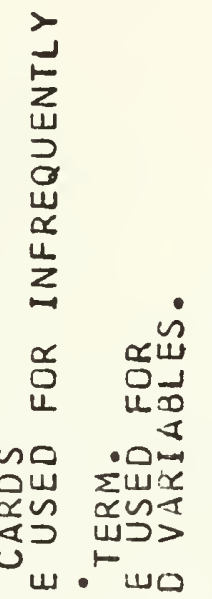

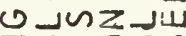

Zळ山0心0

- $41-4<2$

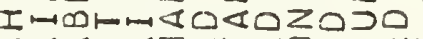
I

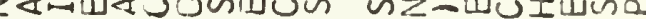

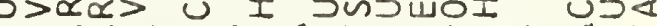
a $\varangle \alpha>$ L  nis Unim

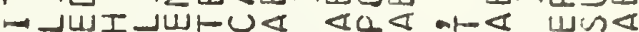

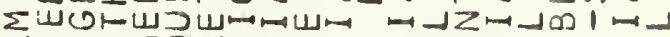

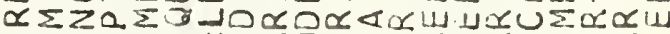
山<

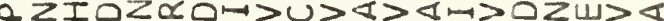
$\left.\sim\|\|_{\|\|}\left\|_{\|\| \|}\right\|_{\|} 0_{\|}-{ }_{\|}{ }_{\|} z_{\|}\right\rfloor$

$\sim$

$\geqslant I$

ar

\&.I

$00 \times$ zo $\overrightarrow{0}$

$\sin \vec{x}$

ind

$\leq \alpha z$

$><0$

$\infty$

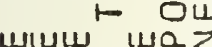

II $2 I \sum$ in

$<$

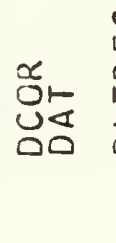

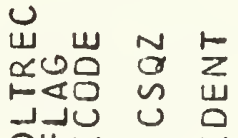

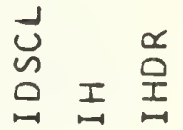

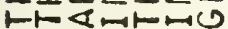





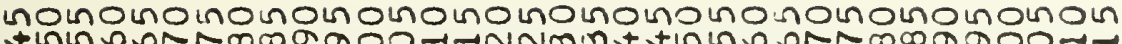
thana

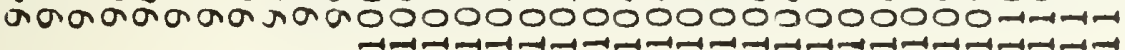

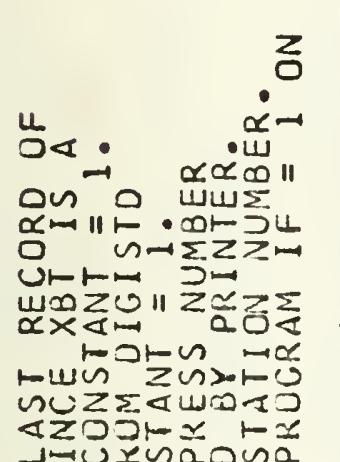

Juxuagua

ง $4 \geq \sum u$

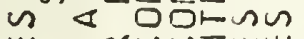

แ $\propto U \cup \geq W 4$

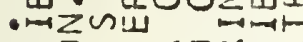

$\sum<$

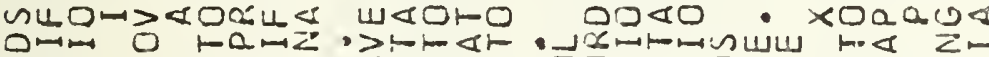

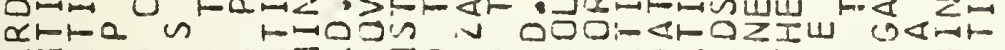

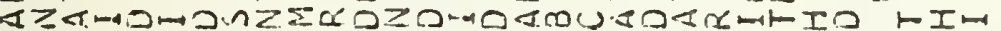

Jur 1 ut омшس גง $Z_{0}$ UIU

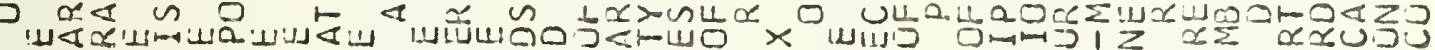

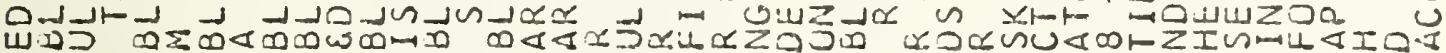
WID

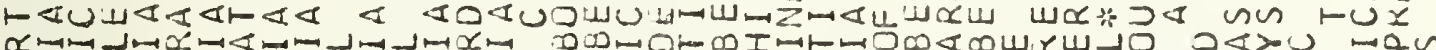

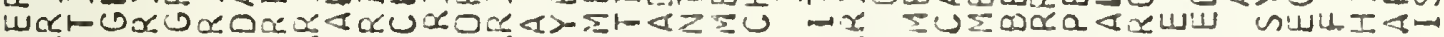

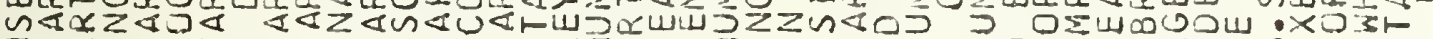

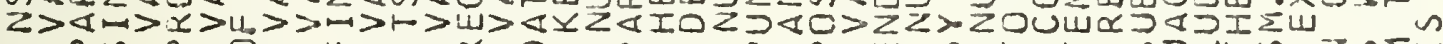

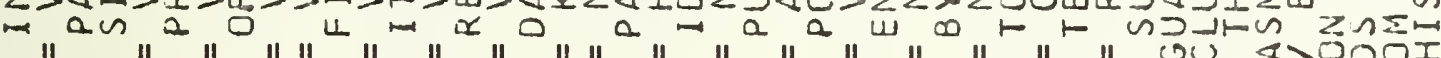

" " " " " " " " " " i ZZIVᄂ wour

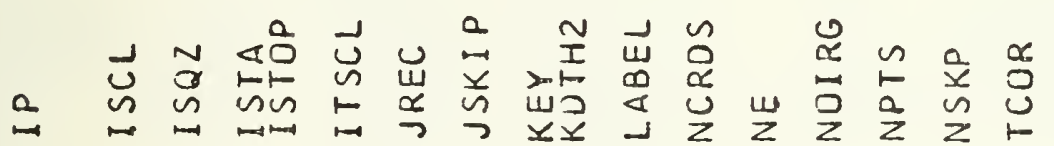
I:

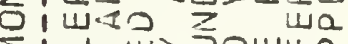

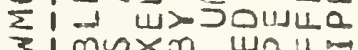

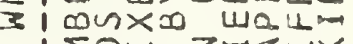

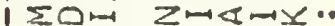

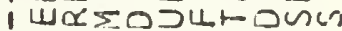
i n⿺ यद् i vums o.

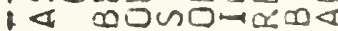

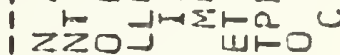
I ZZயOJ 1 - $=4$ L

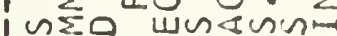

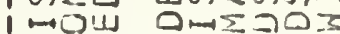
1 U

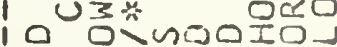

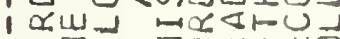
1

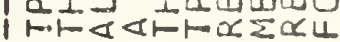





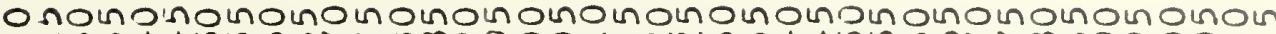

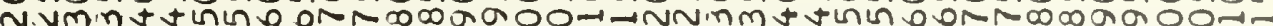

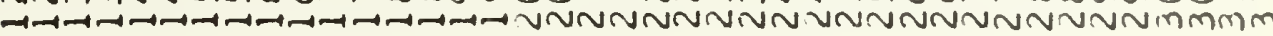

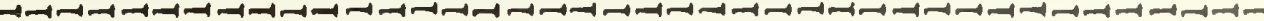

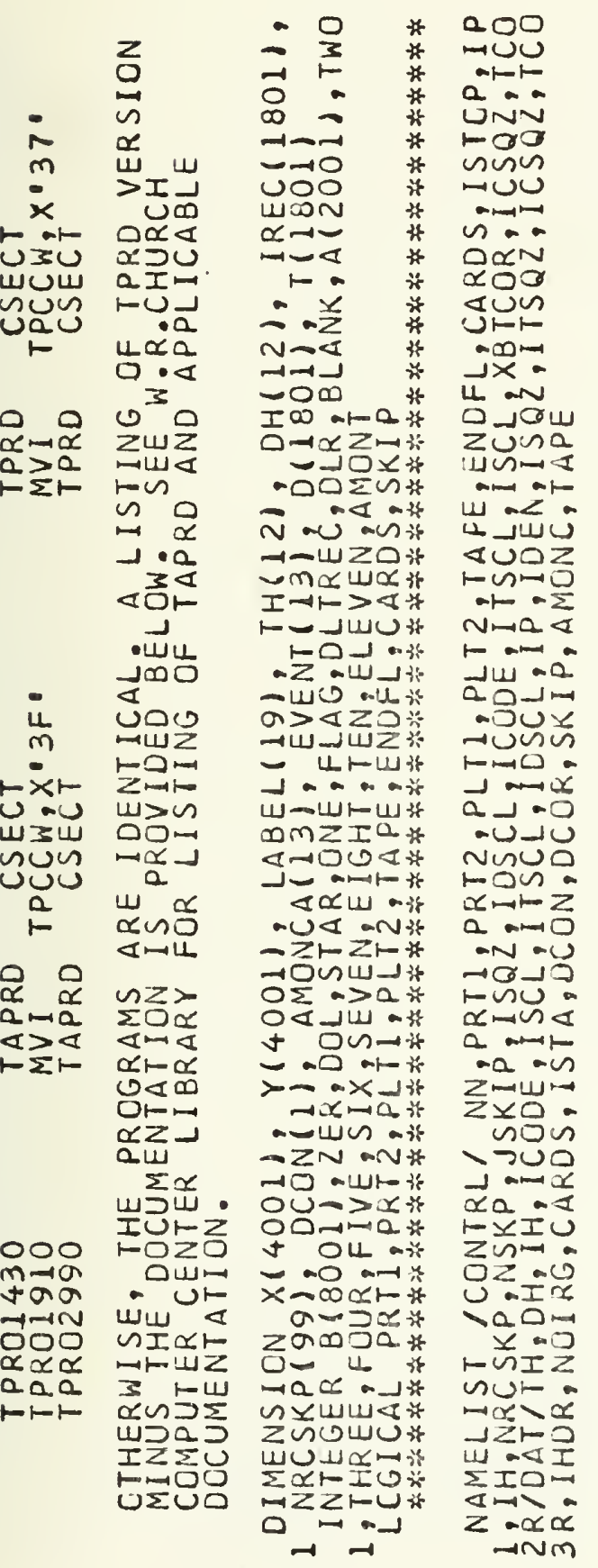

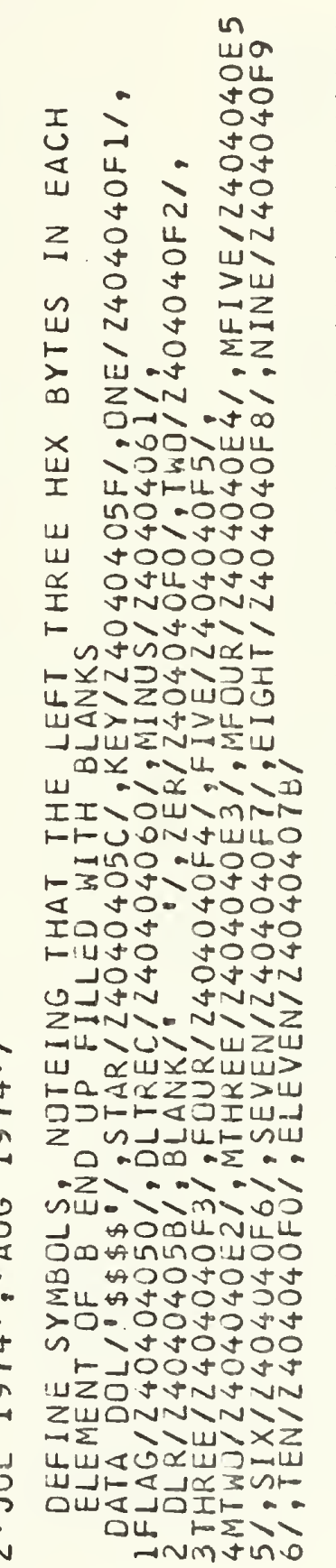

$\because N$

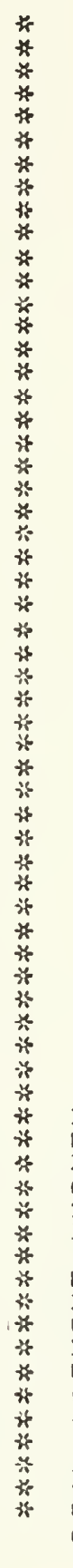

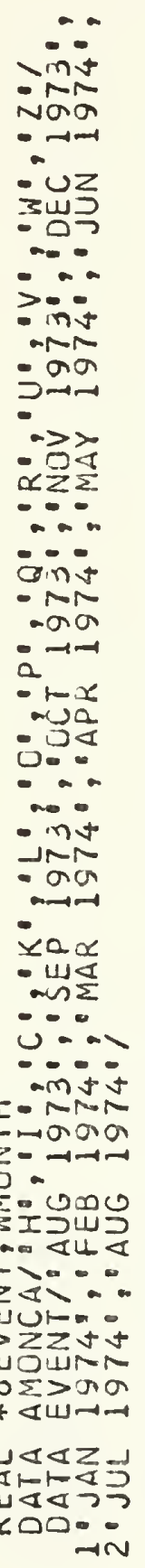

vo טu us

\begin{tabular}{|c|c|c|}
\hline 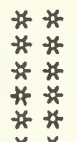 & & $\begin{array}{l}\text { No } \\
\text { va } \\
\text { vo }\end{array}$ \\
\hline * & & $\operatorname{ars}$ \\
\hline * * & $\propto$ & $>\rightarrow$ \\
\hline 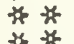 & w & $9 \rightarrow$ \\
\hline $\begin{array}{l}* \forall \\
*\end{array}$ & $Q$ & W= \\
\hline$\ddot{*} \ddot{*}$ & $\sim$ & $0>$ \\
\hline H & IU & ON \\
\hline 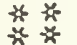 & I & $\bigcup_{n=\infty}^{\infty}$ \\
\hline$*$ & z & \\
\hline * 蓓 & मi & \\
\hline $\begin{array}{l}* * \\
* \\
*\end{array}$ & 40 & \\
\hline 范 & $0 \propto$ & \\
\hline $\begin{array}{c}37 \\
36 \\
3 *\end{array}$ & « & \\
\hline 论 & $\stackrel{\ominus}{\mathrm{U}}$ & \\
\hline$x^{6}$ if & $\alpha \vdash$ & \\
\hline$\ddot{*}$ & 04 & 山く \\
\hline से 35 & $\geq 0$ & no \\
\hline $\begin{array}{l}73 \\
* 7\end{array}$ & $P_{U}$ & $\begin{array}{l}10 \\
10\end{array}$ \\
\hline 徣 & $I$ & $4 \infty$ \\
\hline 莗 & $z \leftarrow$ & \\
\hline 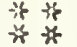 & $n>$ & נa \\
\hline$\ddot{z}$ & ヘ́n & $\vec{u}$ \\
\hline if $\sim$ & 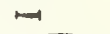 & 0 \\
\hline if > $>$ & z & Z \\
\hline 话 & ๔ய & wo \\
\hline$\frac{\alpha}{\alpha}$ & 뭉 & - \\
\hline$x<$ & U山 & 0 \\
\hline K & $\varangle \alpha$ & 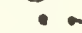 \\
\hline ํㅡㅇ & $u \propto$ & 0 \\
\hline is $Z$ & w & \\
\hline 论 $<$ & $z>$ & $\propto$ \\
\hline $\begin{array}{l}x \\
\text { if } \sim s\end{array}$ & 00 & ? $\alpha$ \\
\hline U & ทய & 口I \\
\hline H. & $x \infty$ & $a$ \\
\hline 30 & w & \\
\hline$* \sigma$ & $\geq>$ & \\
\hline ** & $z=$ & wo \\
\hline$\alpha$ & $0 \sum n$ & $\backsim 0$ \\
\hline 8 & $U N$ & $\lambda I$ \\
\hline$>$ & & $\leftarrow$ \\
\hline & $0 \rightarrow 0$ & $u$ \\
\hline 计山 & z & \\
\hline N & $\mapsto \bullet$ & \\
\hline- & 31 & $\sim$ \\
\hline ـ & 0 & 0. \\
\hline$<$ & $-1 u$ & $\alpha$ \\
\hline 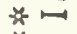 & 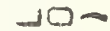 & $\measuredangle \sim$ \\
\hline$\vdash$ & $0 \rightarrow$ & 30 \\
\hline n & Uட & \\
\hline$<$ & $\mapsto Z$ & $\varangle$ \\
\hline$\equiv$ & wZठ & 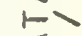 \\
\hline & $I \supset U$ & $\varangle r$ \\
\hline & 10 & 0 \\
\hline I & & \\
\hline & & \\
\hline is & & \\
\hline & & \\
\hline
\end{tabular}

na

$4-$

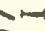

$\rightarrow$

(1)

Ua

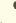

-

$\infty$

U

$\rightarrow$

no

$1 \infty$

in

(1)

2

10

a

0 -

20

$\triangle \alpha$

บำ

ma

-

UI

1.

$-1$

1

In

-

>

12

1

טuvur טنטuטu 



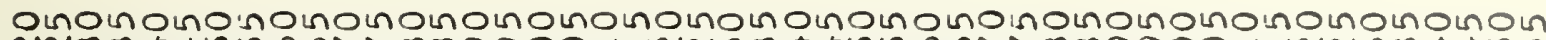

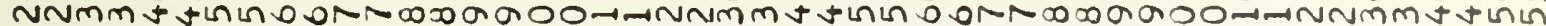

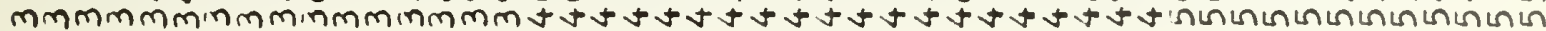
-

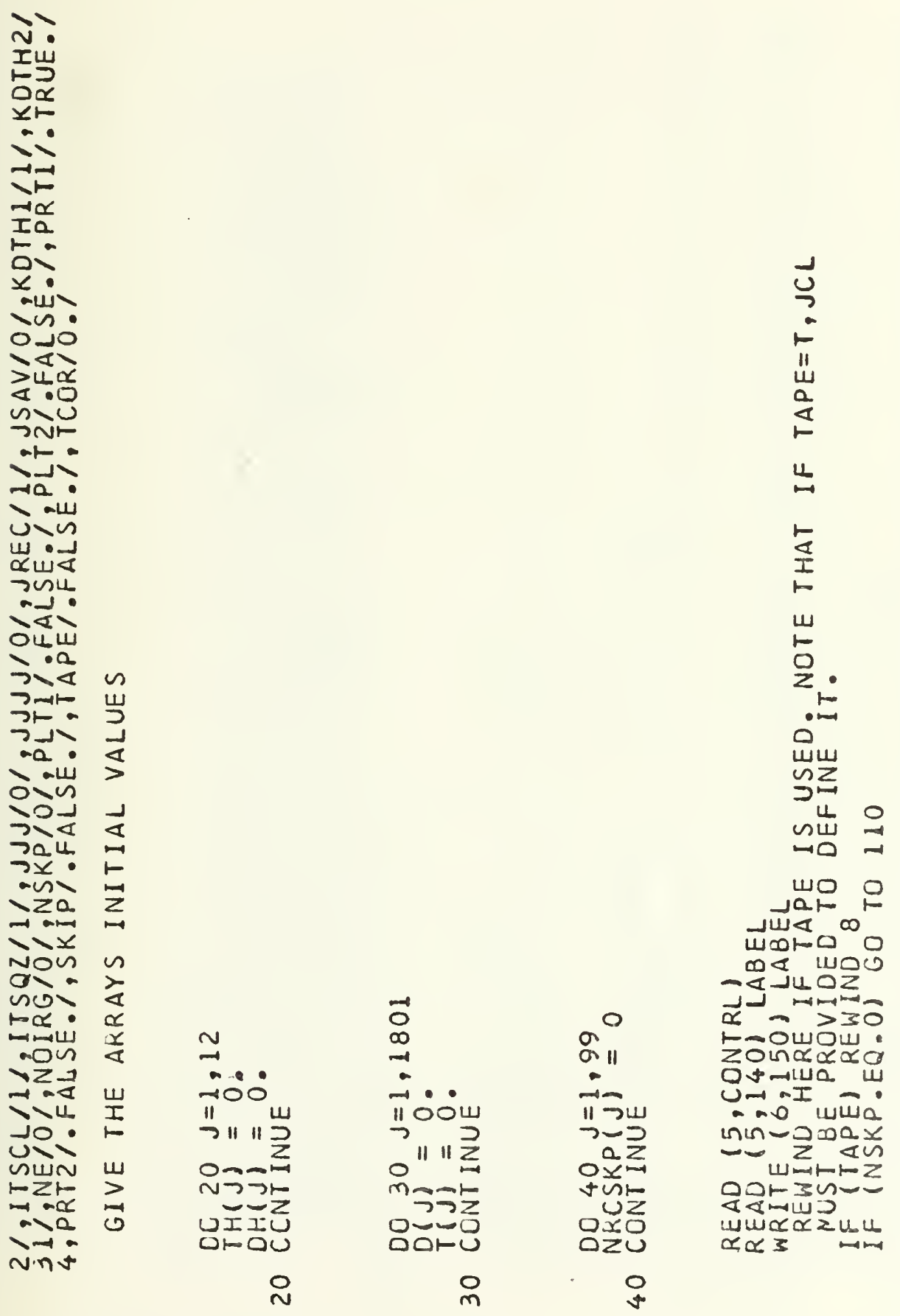

$* *$
$* *$
$* *$
$*$
$* *$
$* *$
$* *$
$*$
$*$

J Zut 스 $\rightarrow$ I 《xด्ว -inz $2 \times$ $10<0<$ m uno żowa $\rightarrow$ wᄄm $z 0$ 중 山-口」0 wa XOQ उxजZะu tus nus ununenz $\infty \propto 0$ $z \propto \alpha \backsim a$ $n=0<$

ш Just

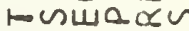
$<0 x-14 x$ $-\alpha$ 전 Zu\& யயா> -

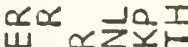
LoOon a U⿺辶u $\omega$ - $x$ $\rightarrow \infty$ axon - $n x z+z$ oDu 11 Ezauoa?

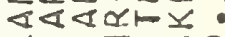
$\alpha$ uz no ง- वZய 00 anu a $x x \mapsto \supset z \cdot a$ ans jum.

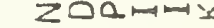

แ山 $\sim \sim$

Inแ⿰⿺乚一匕u

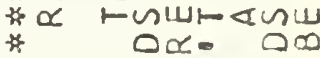

i a $\because \alpha=$ Zu $\alpha$ แดว ज力 WU ดแแดடய $z \propto x a>x=$ I 我 



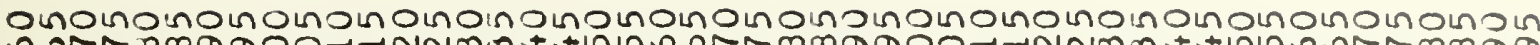

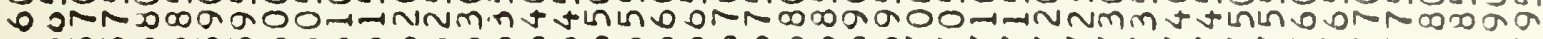

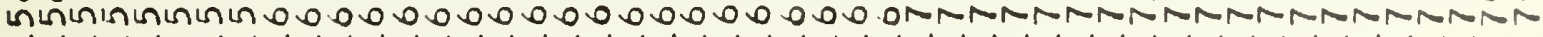

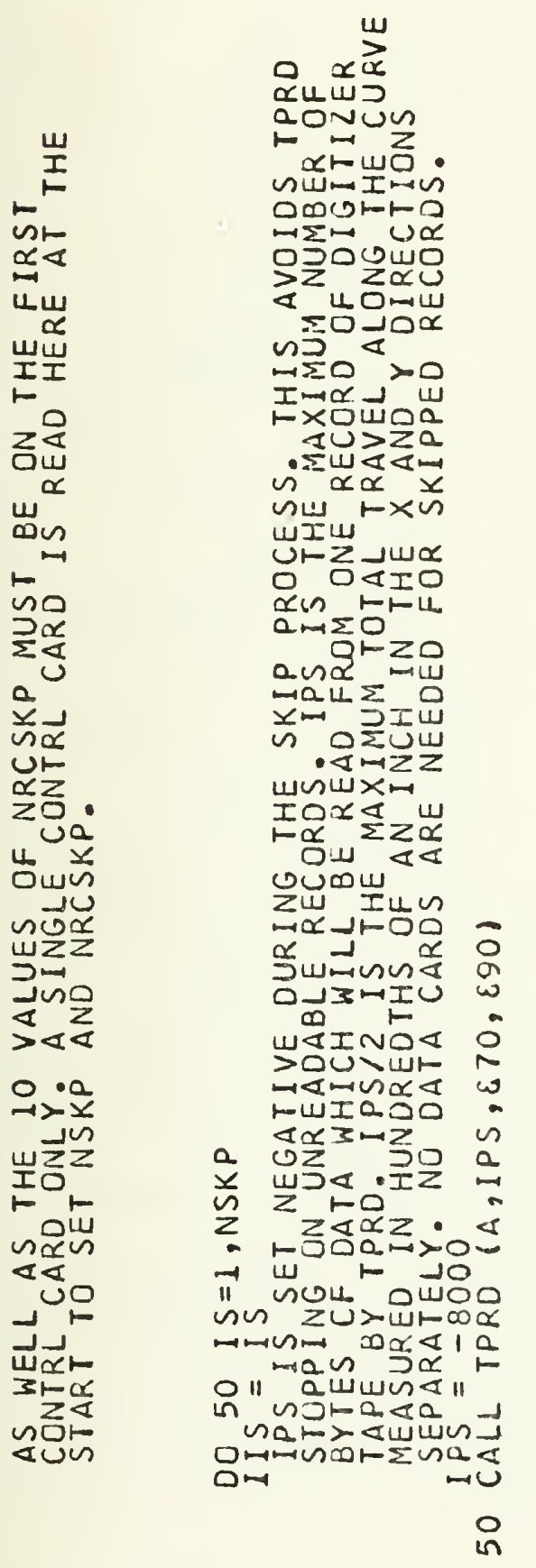

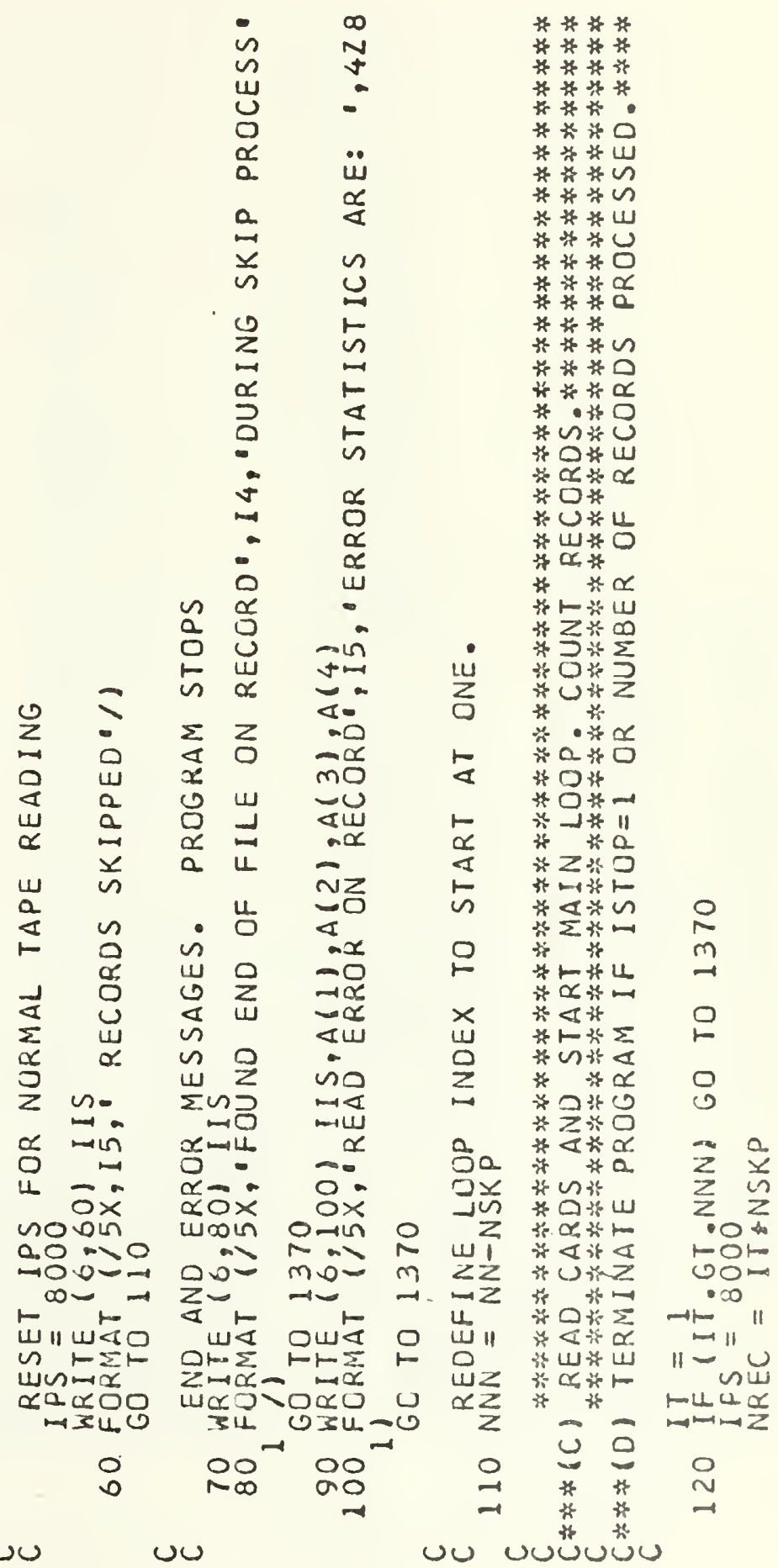





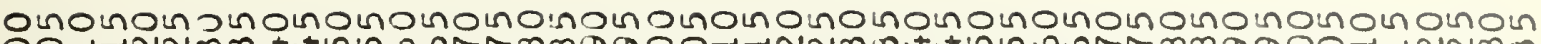

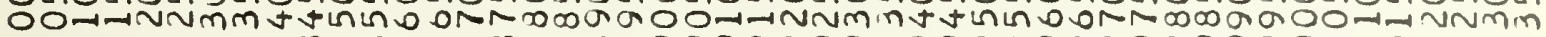
ம

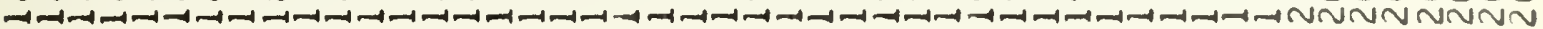

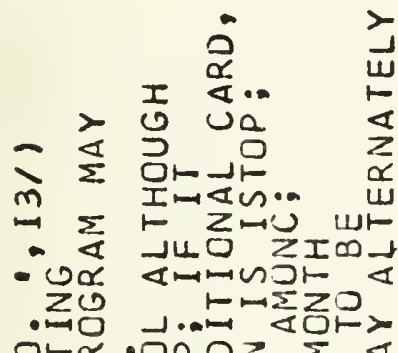

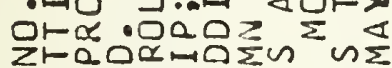
แ

onแ⿰Z幺 $\rightarrow$ IU $\propto$ IU๐つZดZトயZ O্

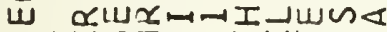

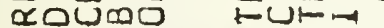
W $\varangle U Z \backsim: U \varangle I$. $\sum_{0 \rightarrow \infty}-1 \sum_{\infty}^{\infty} \infty \sum_{0}=0$

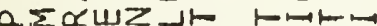

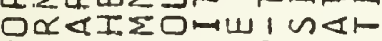
ОயuमJU INUIZ

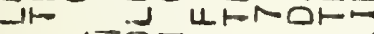

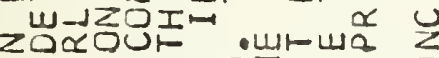

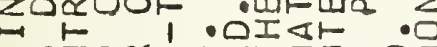
4nzox

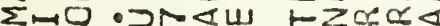
Ut0 $\Psi x$. D⿺辶 to guma nx वшس - I шฉшzس

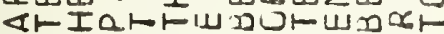

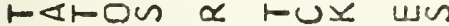

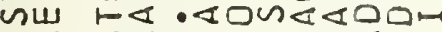
ozn」U थ $x+<4$.

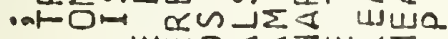

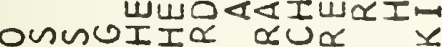

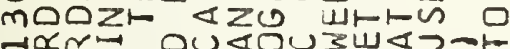

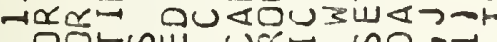

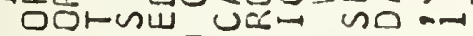

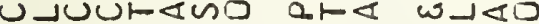

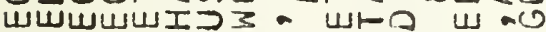

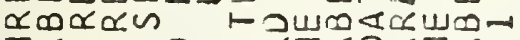
Z< ow $x I<0<I<10$

Ju

0 u z

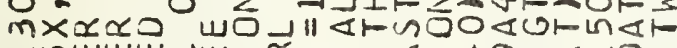

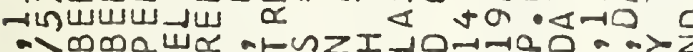

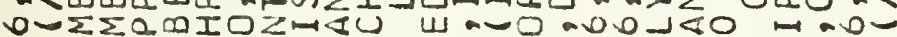

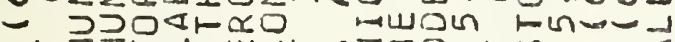
a 111 11 x๐

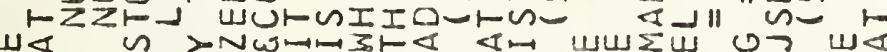

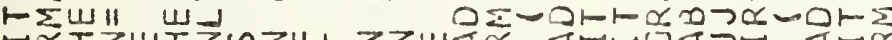

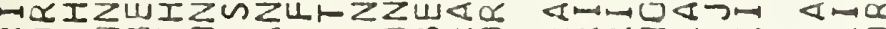

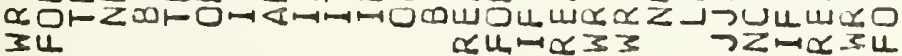

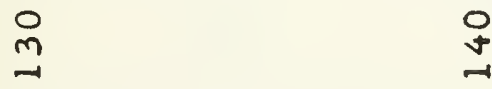

טuטuuxumun

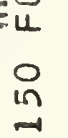

* *

*

** *

* *

$\not \forall$

* *

诺

*

* *

* *

*

获

*3

* 诘

* *

诺

if

*

㻤

*

*

it

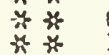

范落

话落

落落

范

话话

* *ै

if

* *

*

落

落

断

落

*

* n

* n

苦

II

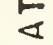

$\stackrel{1}{\alpha}$

wis

$I$

No

12

$<\frac{\alpha}{2}$

トト

또

wx

zZ

I.

$\propto F$

แ $Z$

כ)

$<\geq 0$

I

우

แผก

$\sim u$

जou

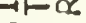

$\Sigma$

$\alpha$ IU

○之に

$4<$

जकะ⿱

4)

0 at

$\rightarrow n z$

$>0$ व

$0 \times 51$

$\alpha<\omega$

Q

$1 Z Z$

SOn-

wOF!

$4 \supset 0$

Zzoㄴ

ㅁu

$\rightarrow \propto-0 \supset$

\|w-IZ

이

$0<0-1$

IUN z

더니

on

*

טư uטur
N

$u$

$-$

ถัก

N

N

U.

$-1$

$x$

in -

n $\infty$

- in

$4 x$

$\rightarrow+$

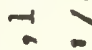

$\times>$

in $\rightarrow$ UाL

$\rightarrow \rightarrow u$

No? <<?

-

Noiv a a

-OW ‥

-100 $-\pi$

ar OOI

XN * NNF

in $\rightarrow$ a $\rightarrow 0$

VII F-1

- io nom

N-x $\rightarrow$ ind

$4 \times \cdots$

tin a-10

-1) $\| 11 x$

anu $\rightarrow$.

$\times \cdot z$ - -4

nN $=2$

Vu $\rightarrow \supset$

NAn -2

그 는?

$4 \times 7$

-1in

an 0000

ar ar 000 a $x \cdot \times-1-1-1-1$

Unt an

$-4-\| 000$

-

รx

(1)

w

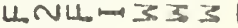

$0^{-1}$

00

$\infty$ a 



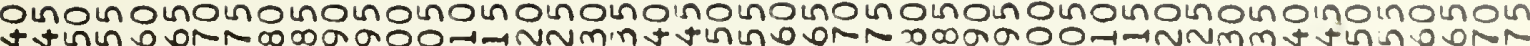

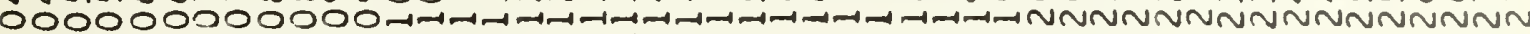

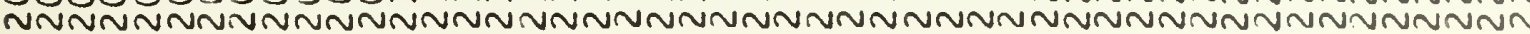

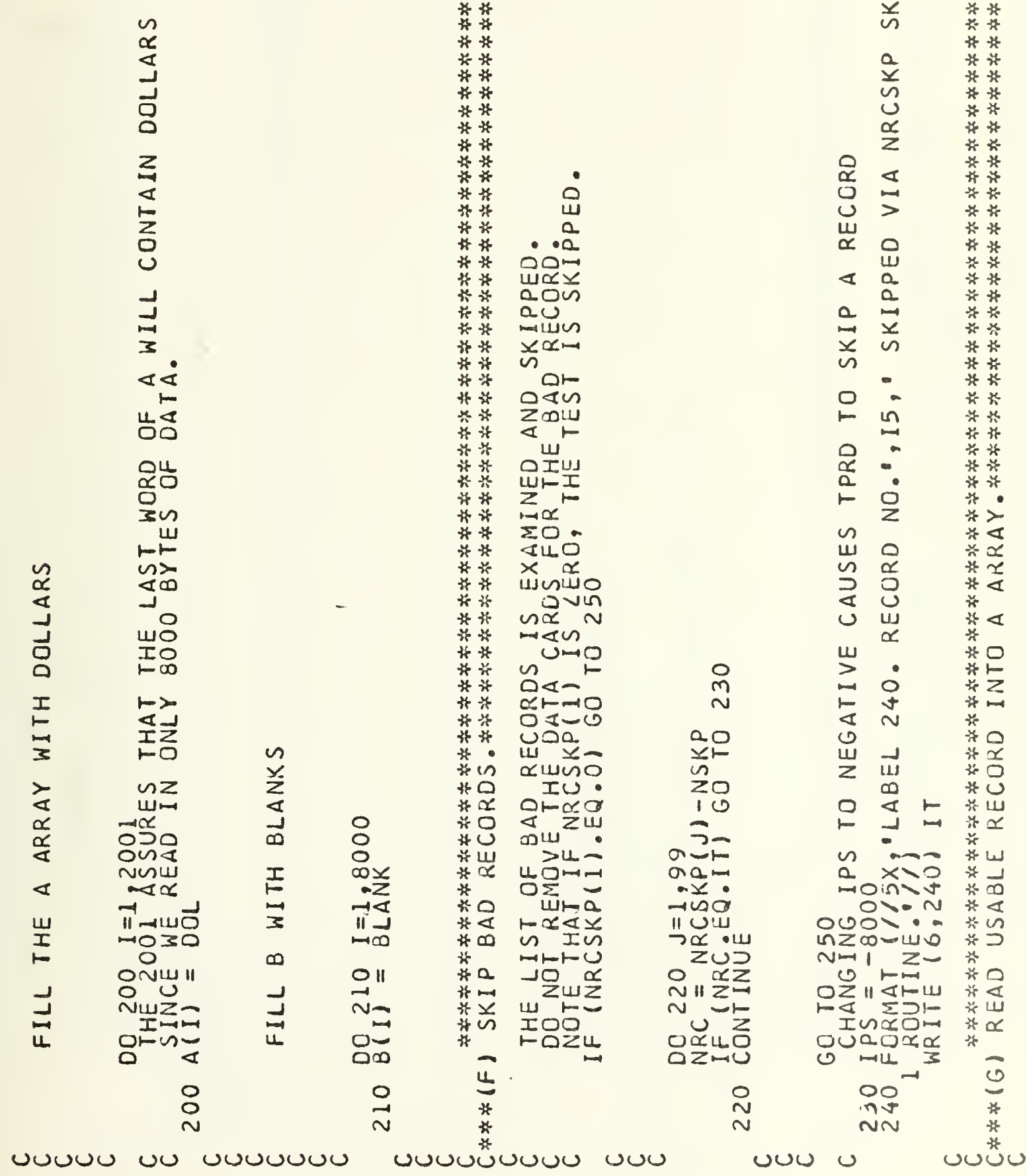





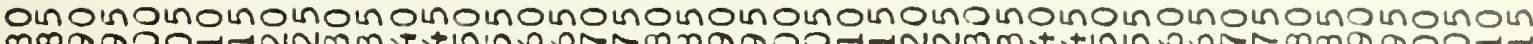

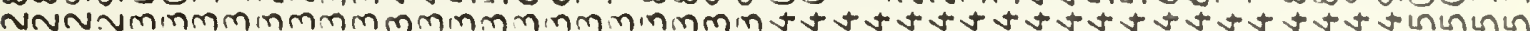

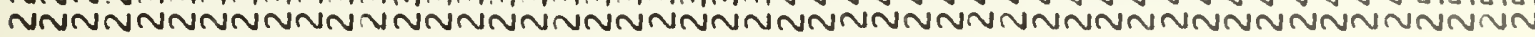

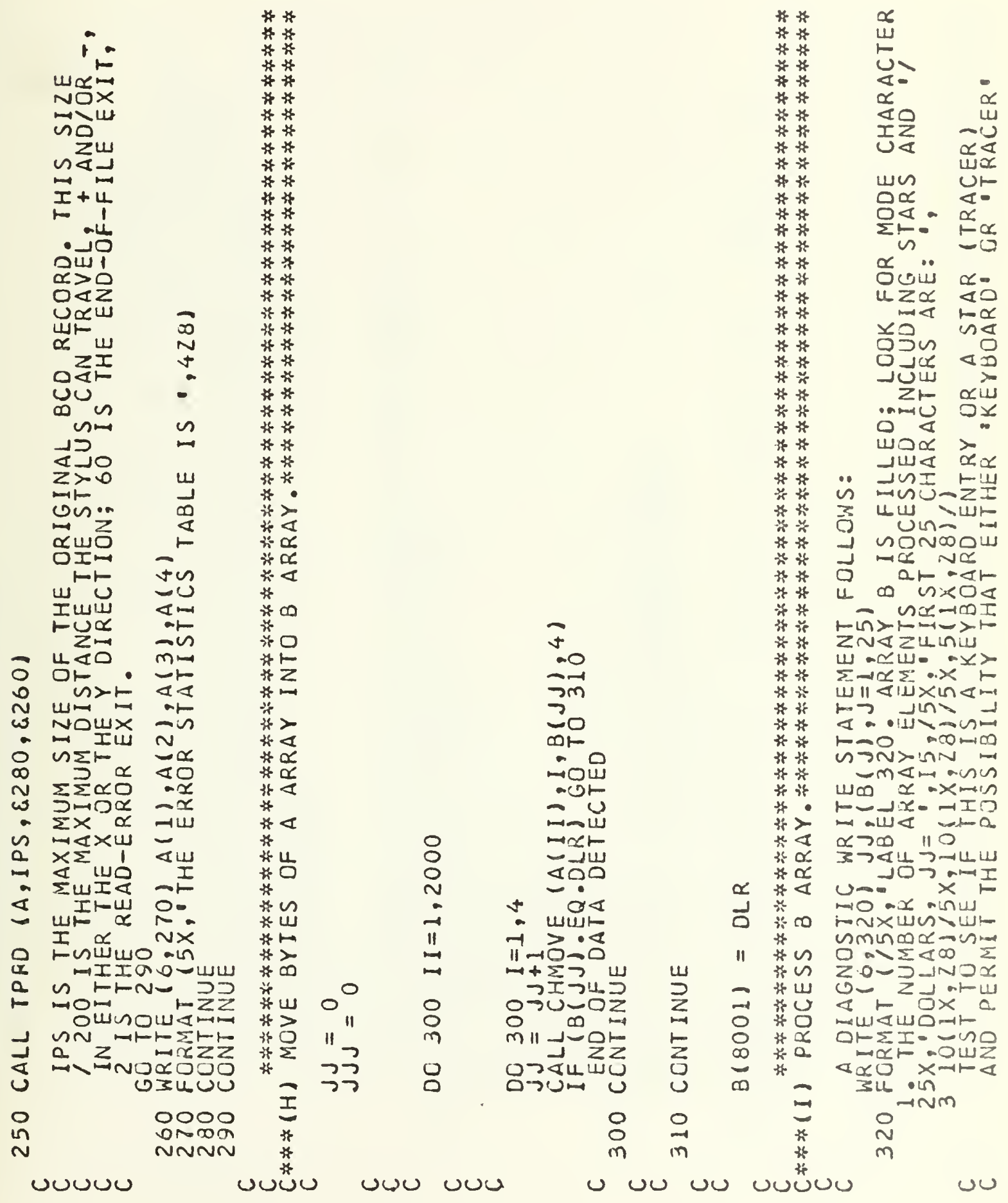





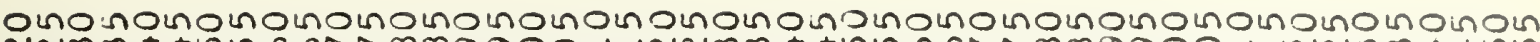

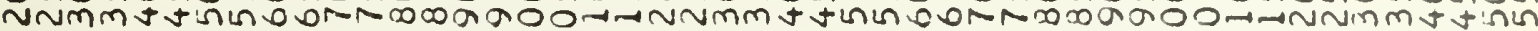
ininininininininininininininininv0.000.0000000000000000NNNNNNNNNN

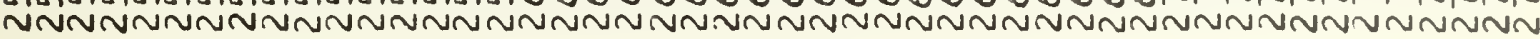

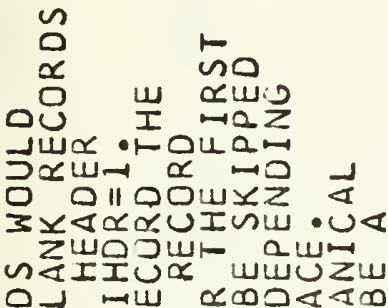
विल uv $u$ ur zorü

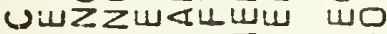

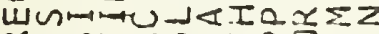

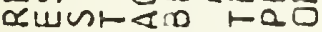
$I F \alpha$.

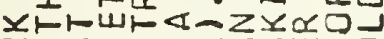
$z$ nn N<iu

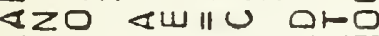

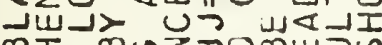

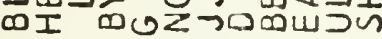
In Zu Z IN

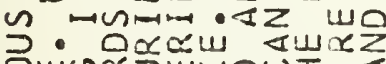

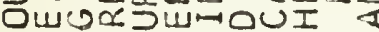

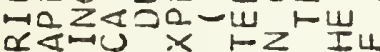

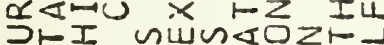
$a-1<\alpha$ \&Un- u

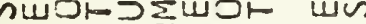
IZIUOH J

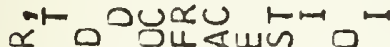
$u \in c \propto \alpha \infty \propto ⿻ \alpha$ NDO-O \& $4 \alpha$ u

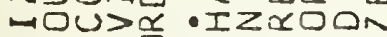

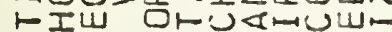

แ

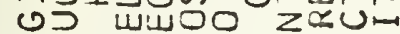

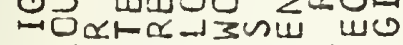
$0 \propto u \propto ⿻ 上 丨$ IDuxu auzuno

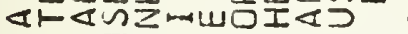
ह. $142<>$ jII-JZLU क IU $\varangle 0$ कणI $\propto \propto$ N.

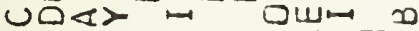

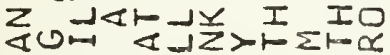

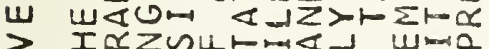

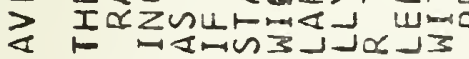

I

$1-\alpha u$ का काष

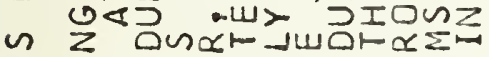

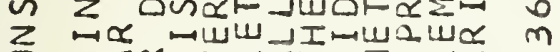

0 i $1 \alpha>d<1>I \quad d x-$

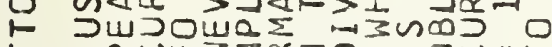

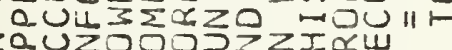

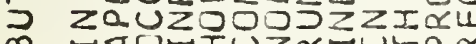

$\infty \leftrightarrow \alpha \cup m$ I

崖

$\sim$

$\approx$

岃

is

जu

ấ

Un

岂。

Oo

แே

जu

딴를

또임

比

$0=4$

$\sum \frac{1}{30}$

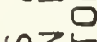

onin

$\varangle \rightarrow 0$

wn ano

$\propto z a m+$

แलx

ziño

ロ【フトト

12 .

จ는ำ

$\propto \sim \infty \infty$

DuगुO

ZI - $-\bar{\alpha} \frac{\alpha}{2}$

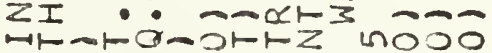
3. OU山म

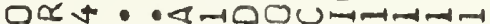

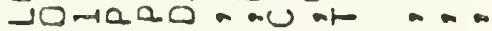
-

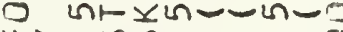

4.

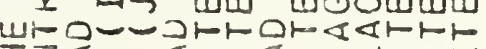
$I Z<\quad$ oा -แแนแแ $\alpha \mapsto-\alpha 33 \alpha 3 \mapsto 333$

m

uv

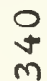

in

i

은

讨

u

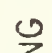

Z

(n)

$\sim$

u

虫

x

$a$

$?$

วิก

$\rightarrow \infty$

떠

Q⿻日土𧘇

$-5$

○- 4

minn

an 00 -

0- T 100 *u

- $\|1\| 7 \&$

$5>-1110$ स

w⿺辶0"

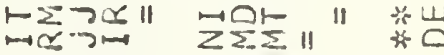

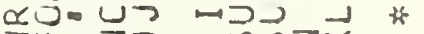
उL Z7 जिएर

$\begin{array}{ll}\mathrm{in}_{m}^{n} & \circ \\ m & 0\end{array}$

su 



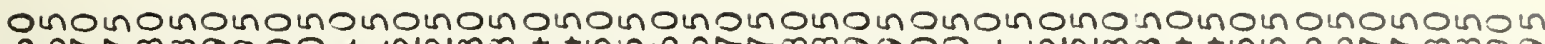

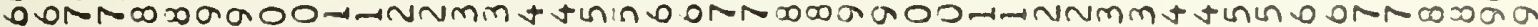

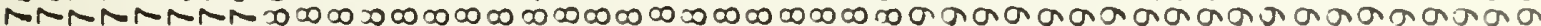

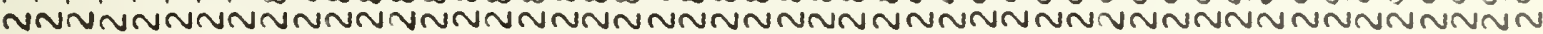

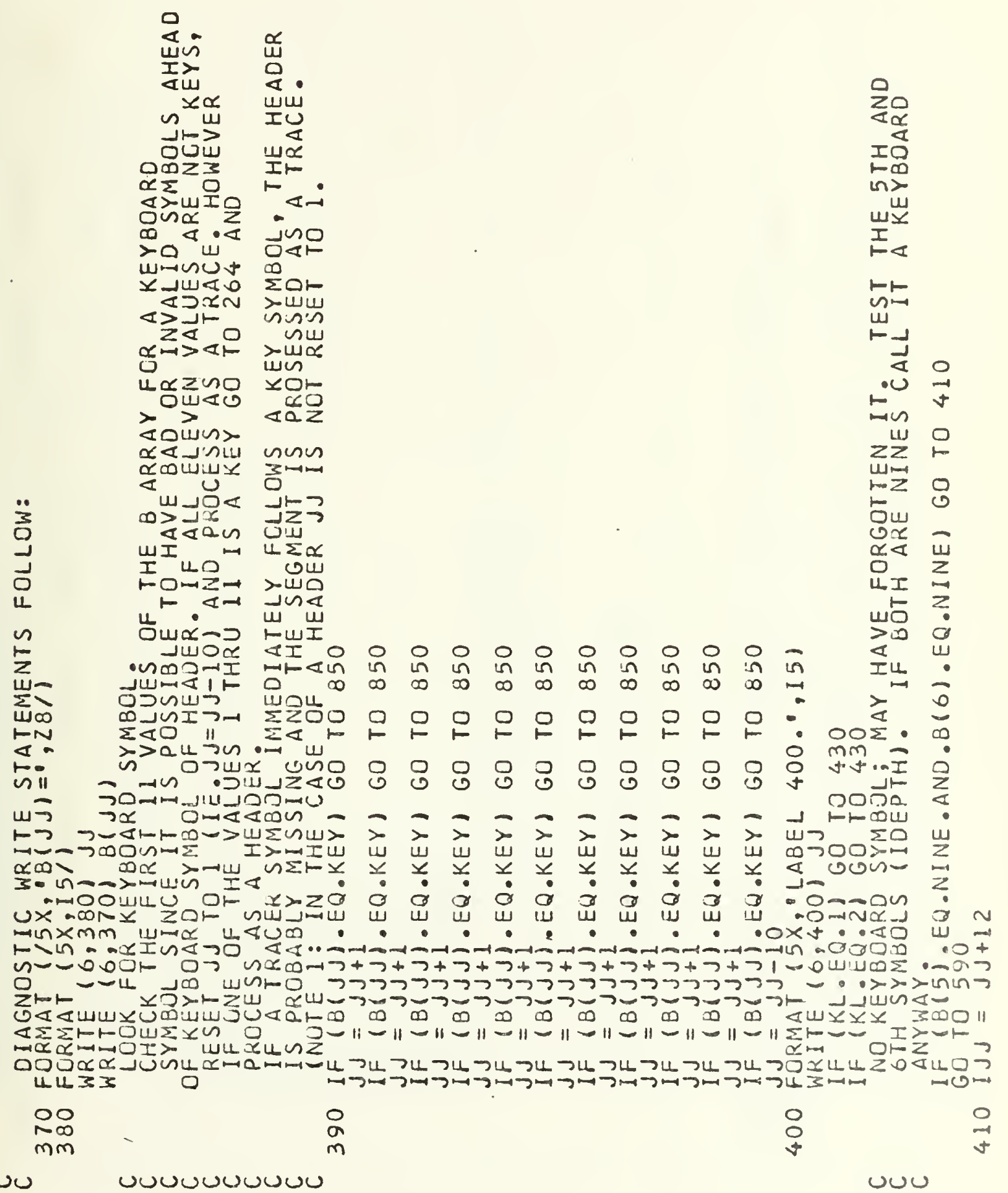





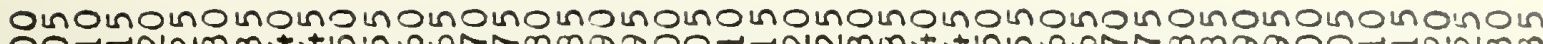

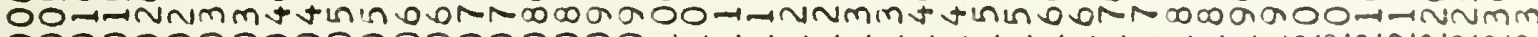

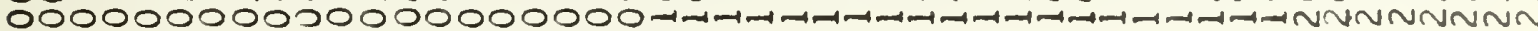

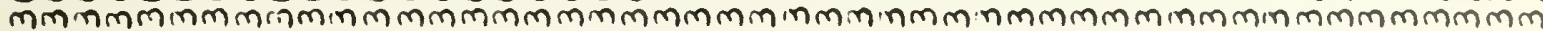

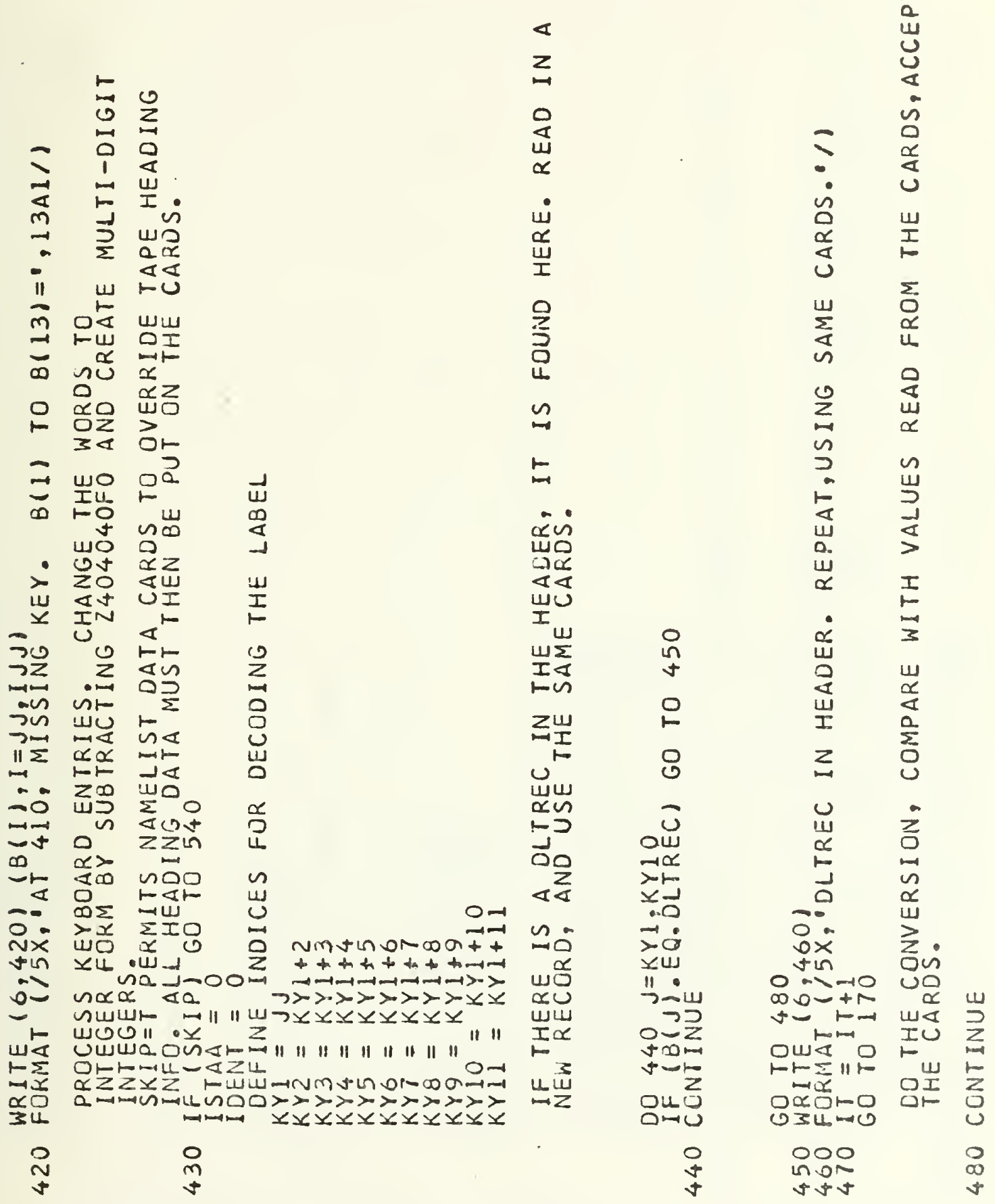





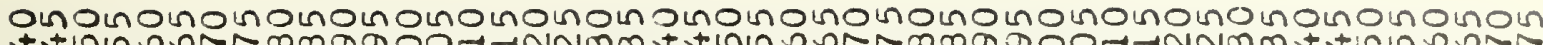

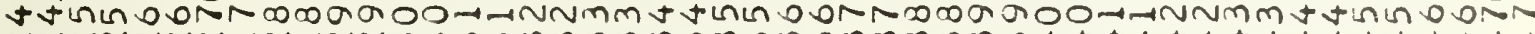

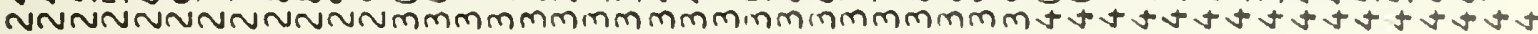

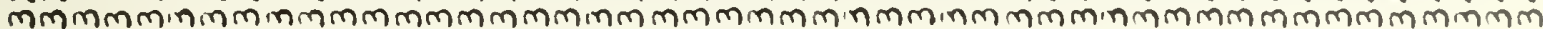

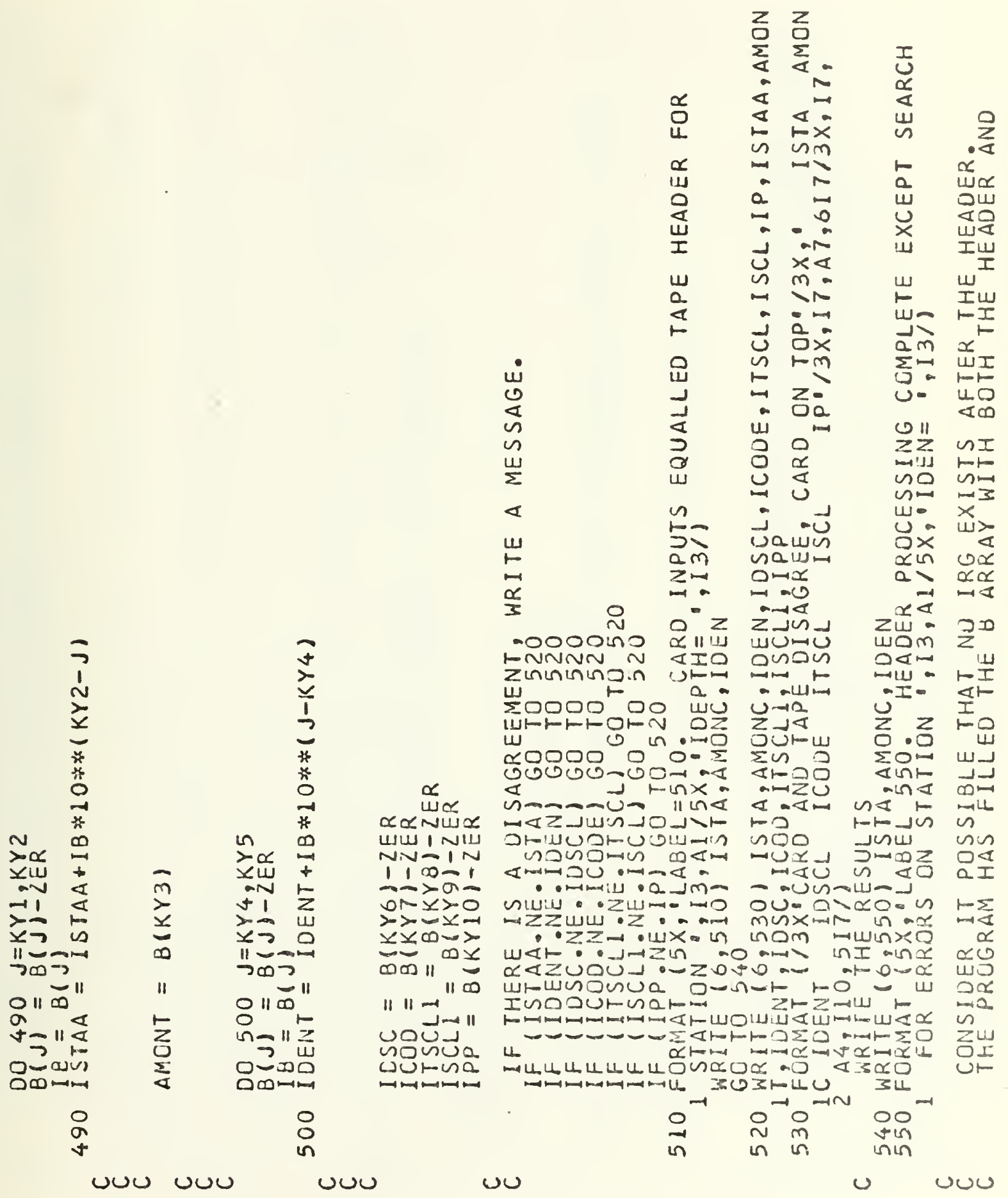





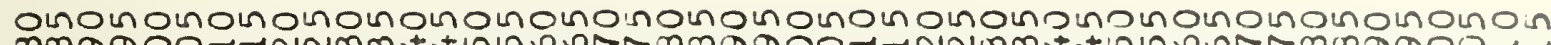

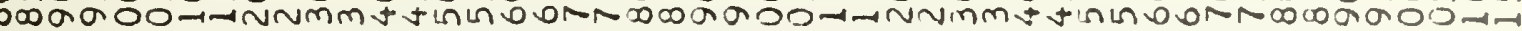
t+t t

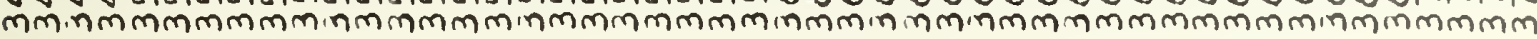

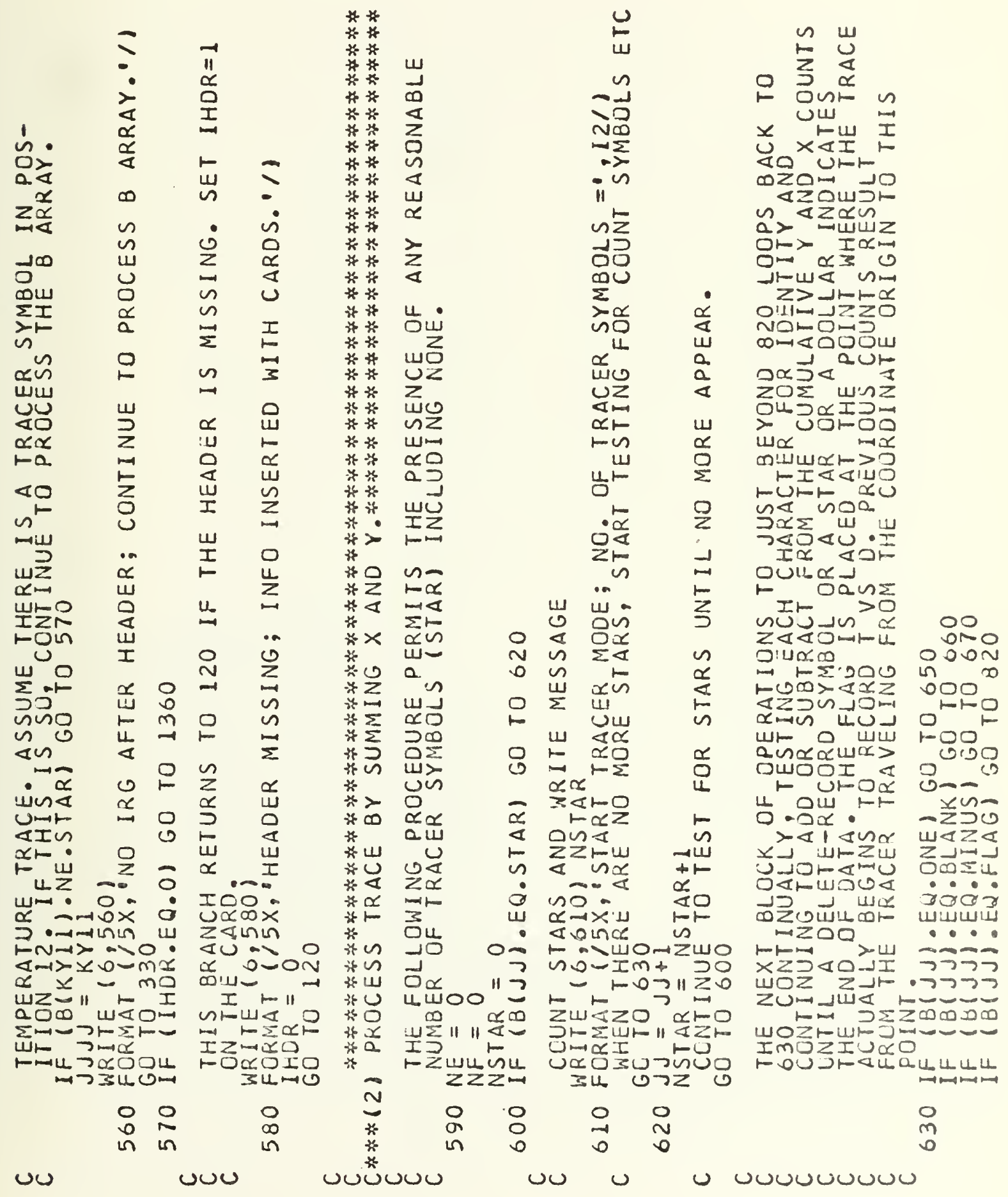





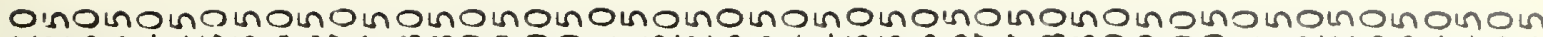

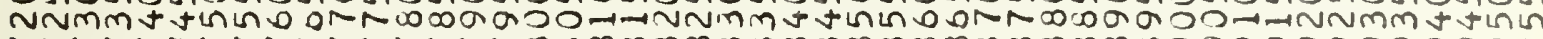

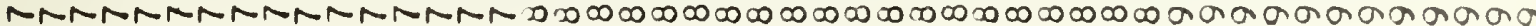

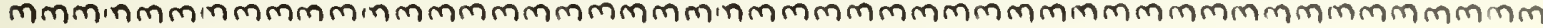

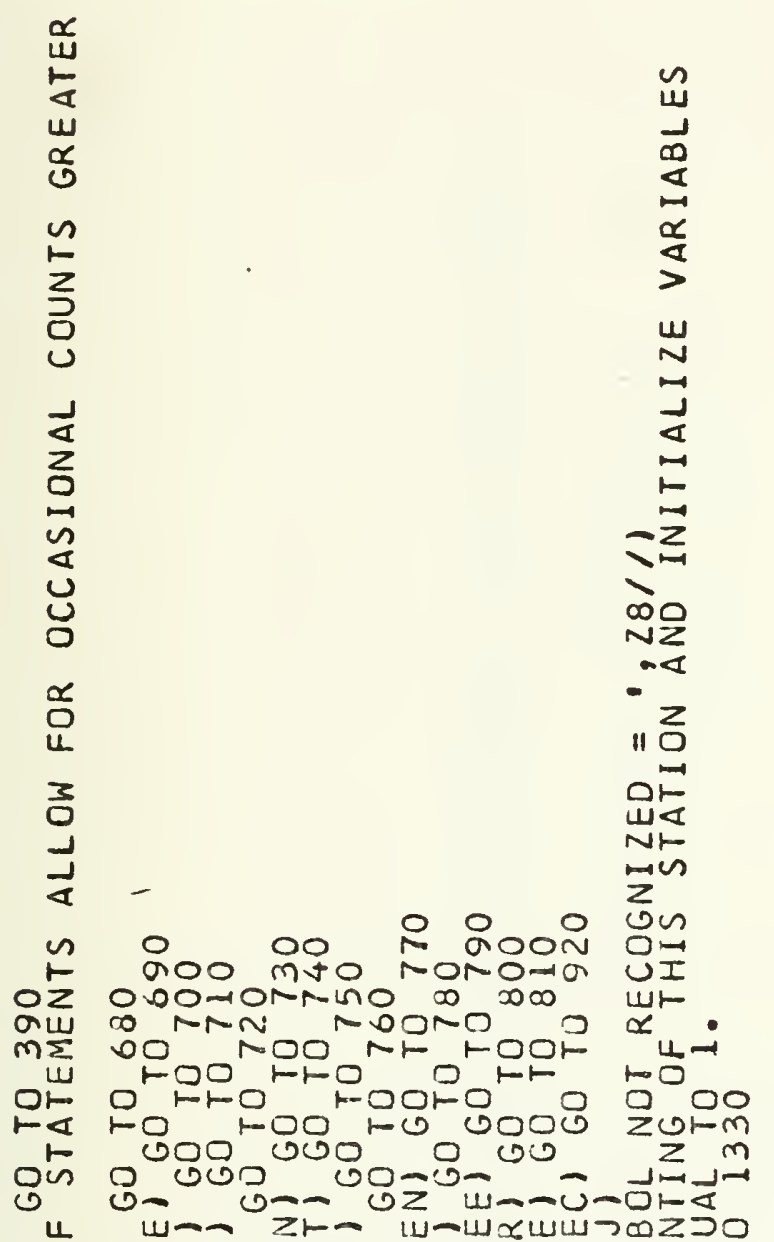

岁

$>\quad 0 \alpha \supset>x>0 Z Z W \leq I 0 m 1->\propto W$

ua :

x马-

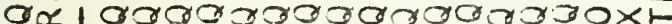

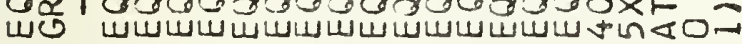

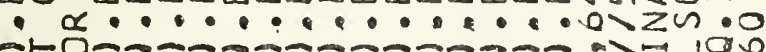

万х

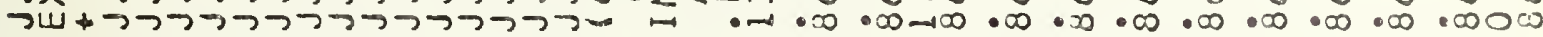
$\bar{D}^{2} \geq 0$ $\infty$ Z II

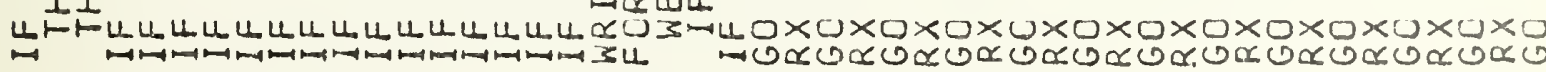

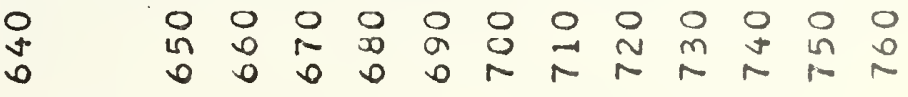

U

$\cup \cup$ 



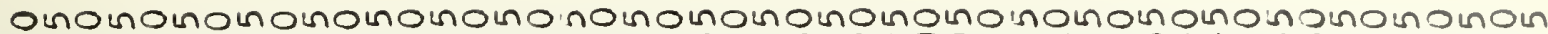
bUNR

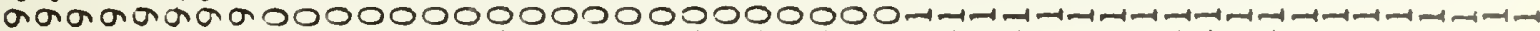

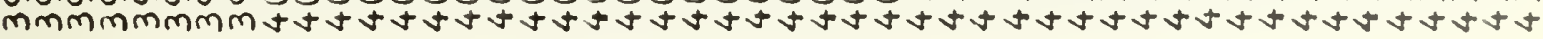

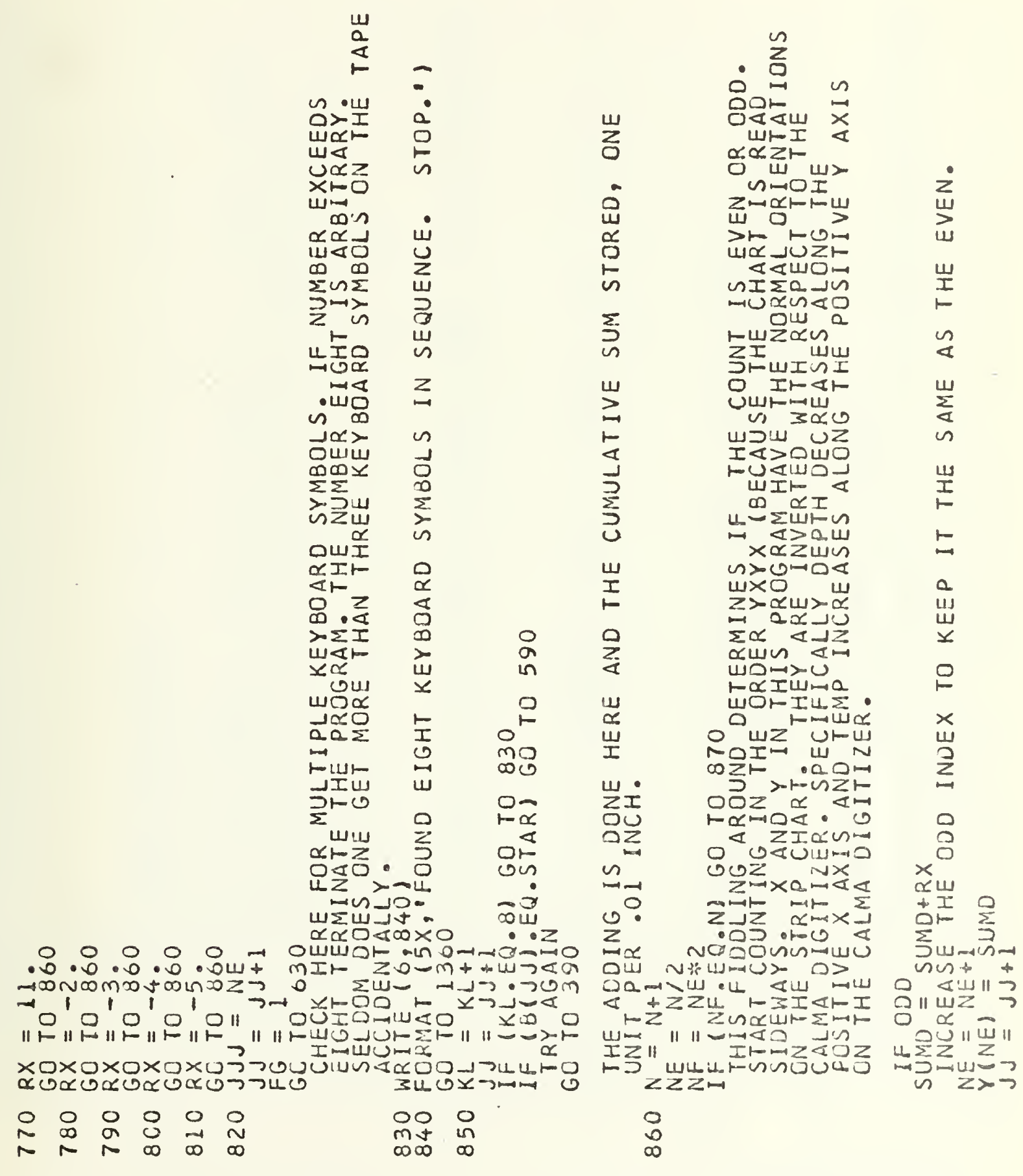

uvu

$\omega \cup \cup$

unumunu 



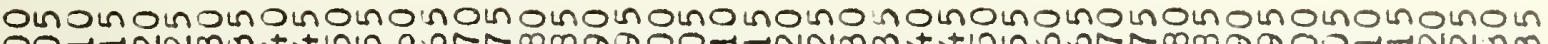

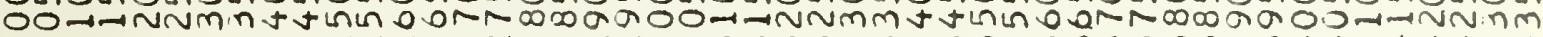

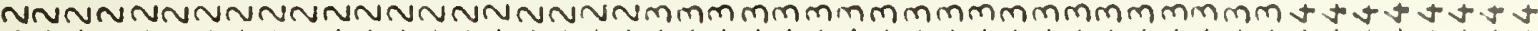

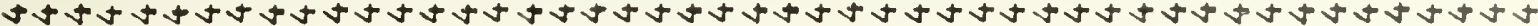

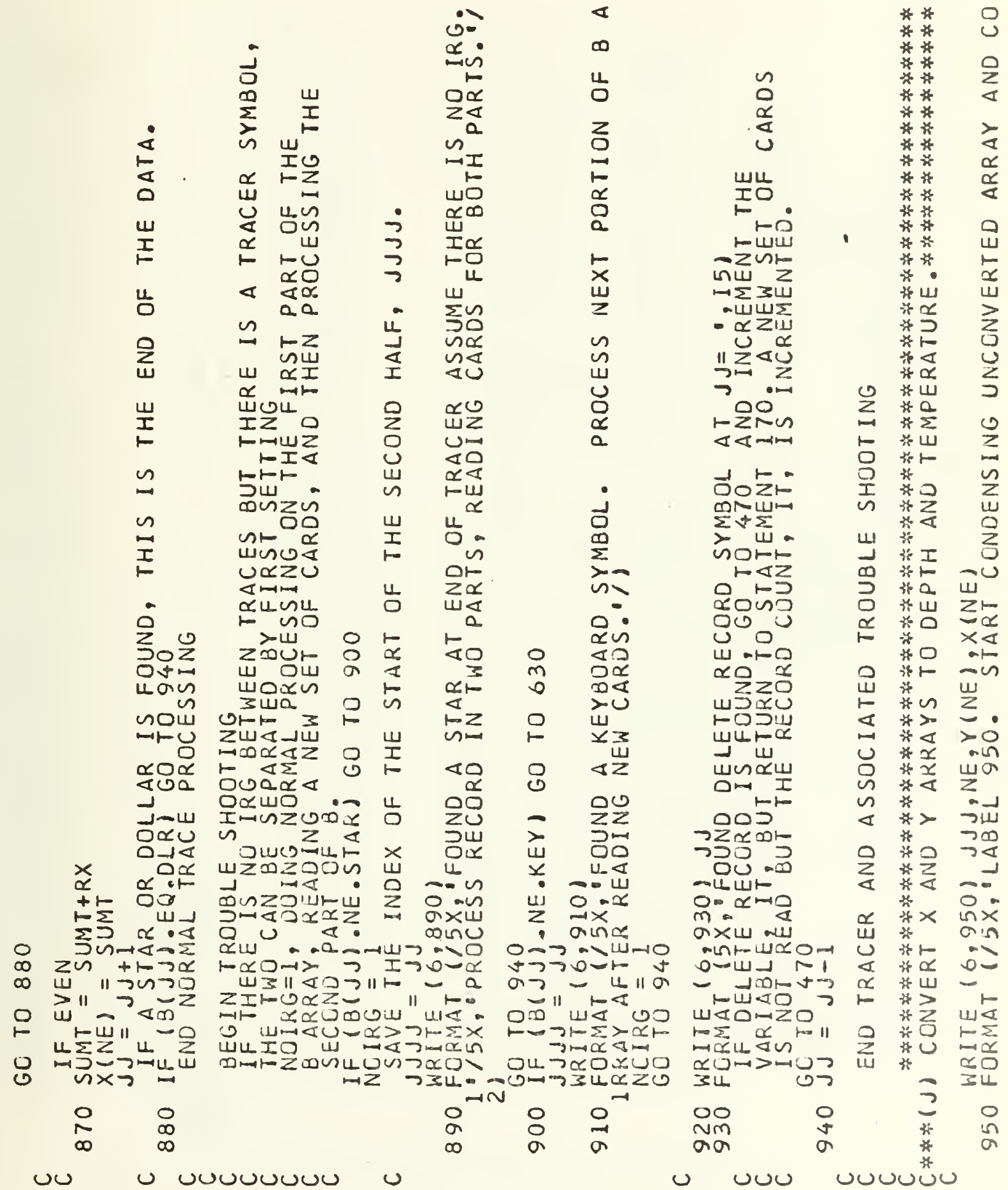





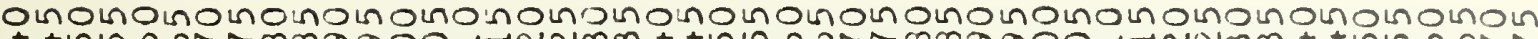
ItULI OUN D D

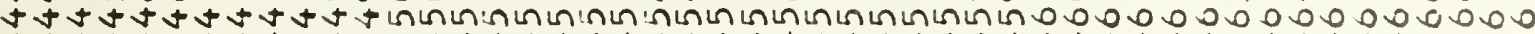

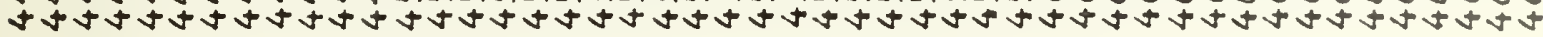

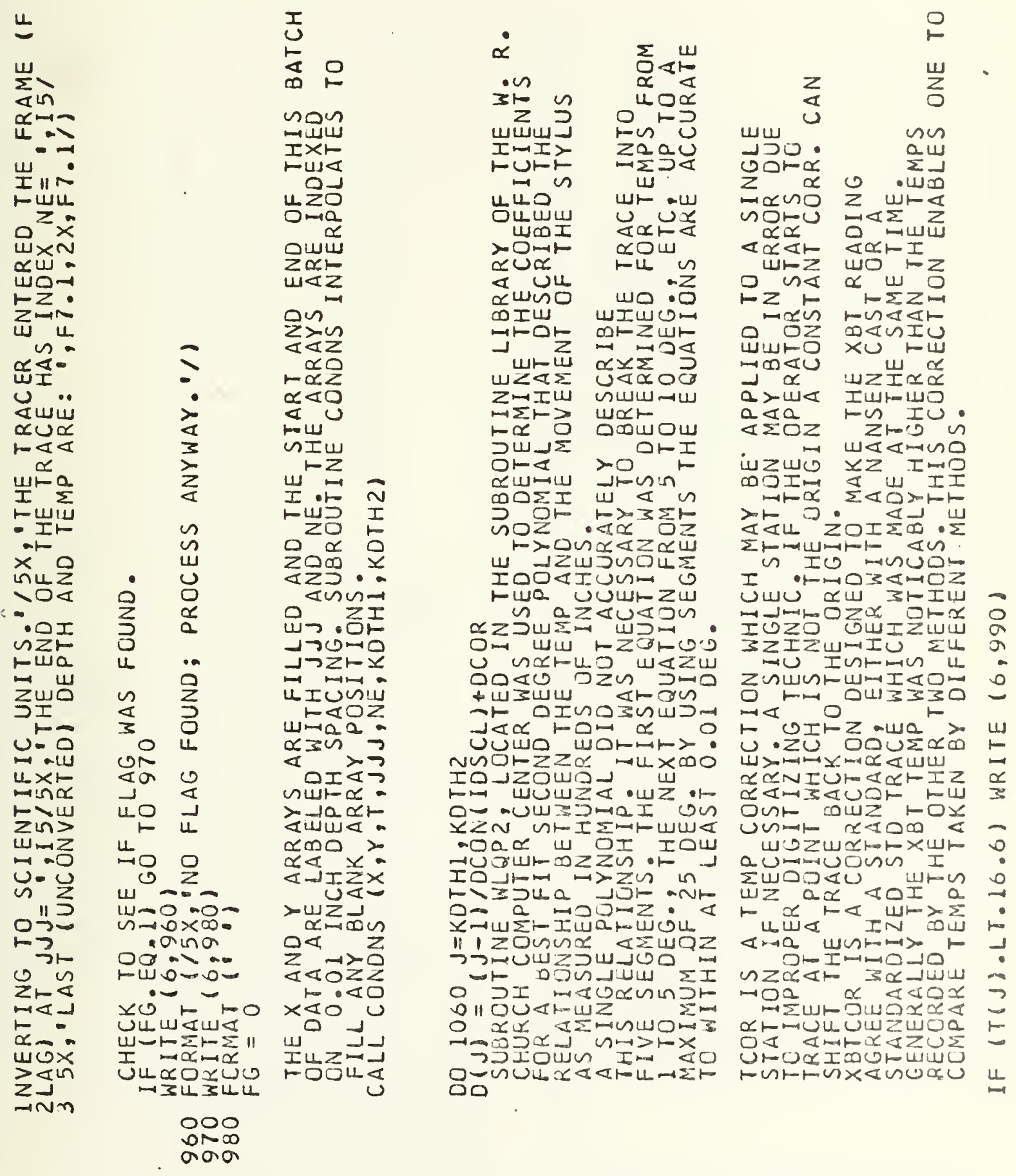





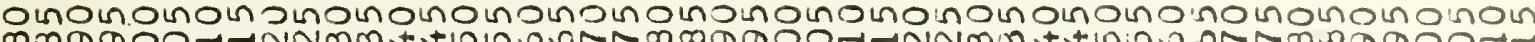

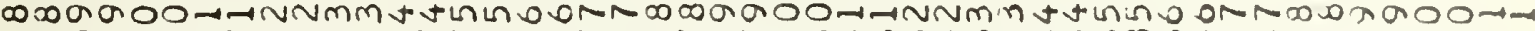

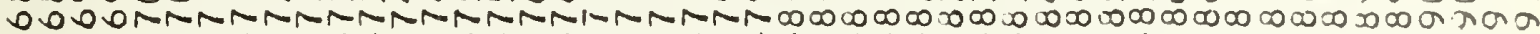
Atatatatatatatatatatatatatatatatatatatatatatatat

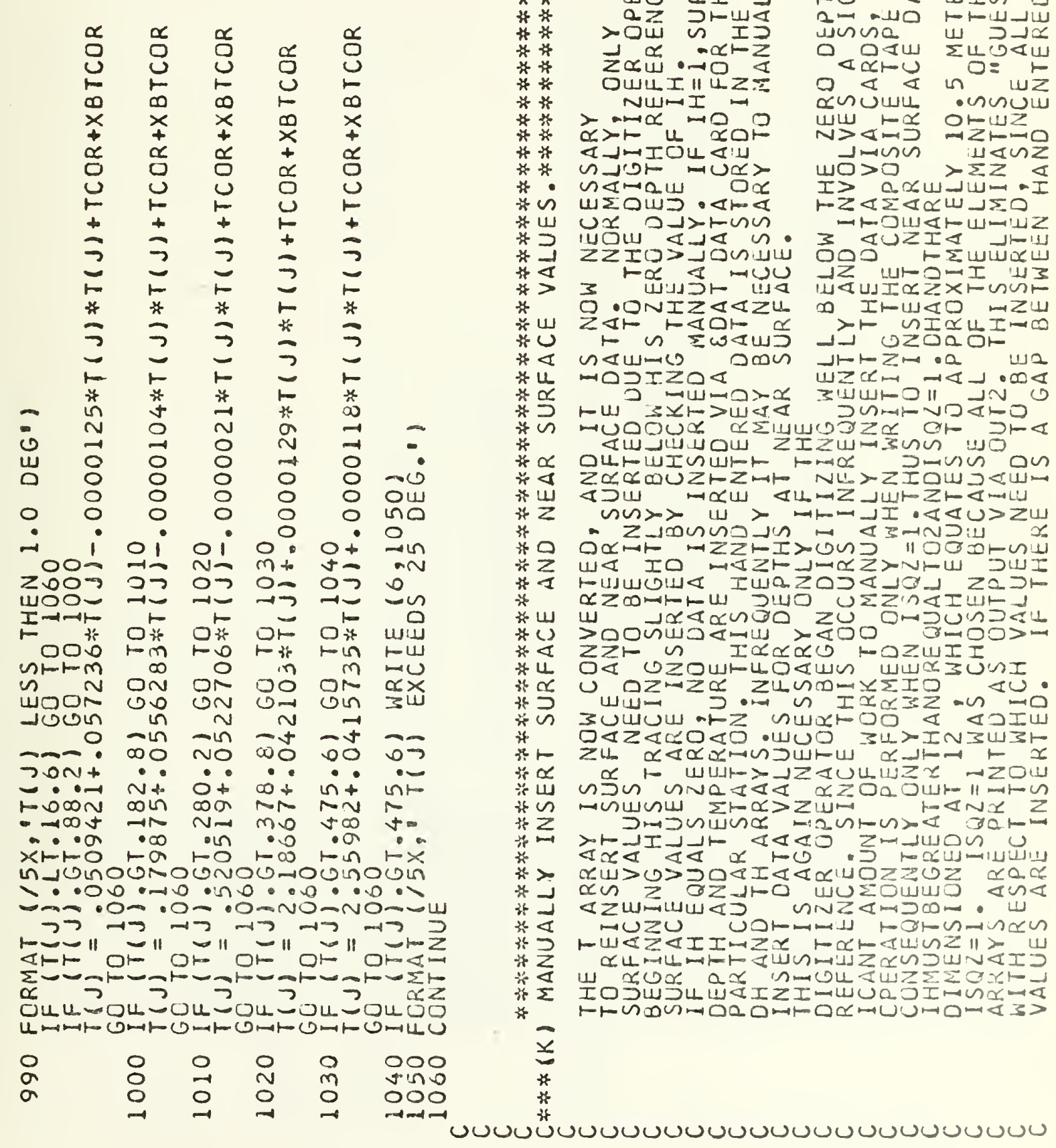





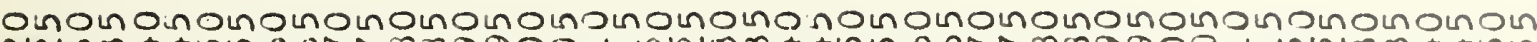

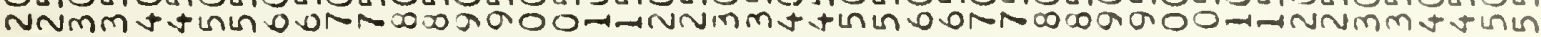

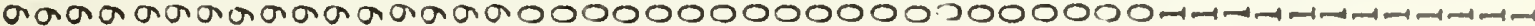

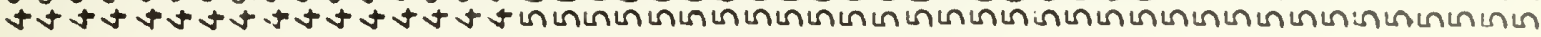

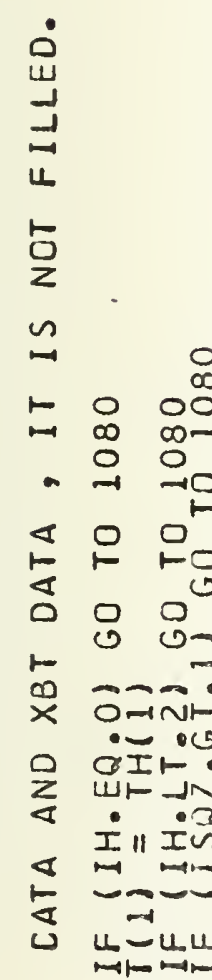

U

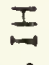

$\infty \rightarrow=$

$11=$

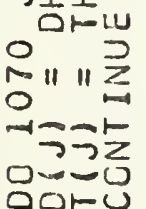

แบ

w

? 3

a.

$\rightarrow \rightarrow 3$

$-\infty$

00 I

Non

$\rightarrow-0$

$\rightarrow d x$

$00 \div$

$\infty \infty 0$

$-1-I$

$\rightarrow \infty$

$-10$

$111 x$

$>>$

$\rightarrow-2$

$\rightarrow \rightarrow$

$-27$

แ1 $\quad 0-3$

$\overrightarrow{1}$

$10-0-1$ 100000

$\omega 1-1-1-1$

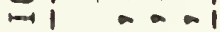

$+111000$

$\sum_{j}^{\infty}$

0 i 4

$2 !$ जиши

\& I J

$-\mid a \alpha \alpha \alpha$ :

$0 \mid-533$

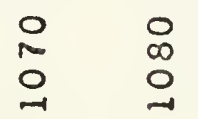

use ${ }^{-1} x^{\prime}$ $\rightarrow$

n 0

$\mathrm{U}=\mathrm{r}$

DI U

Jis

$>T=0$

$\alpha$

$-1>0$

$\alpha$

ग1100

$\vec{n}>\omega<$

$\rightarrow-2$

$\sum \propto \leftarrow$

Soln on on

मी

$1 x-x$

!11แบ

cNuUL

- ᄃ

$>$ U⿺

d-

$-2<$

Zun

แrun

209

CUd-dI

Un⿺𠃊

$\alpha>n$

1.

UடUDZ

$z \alpha+\alpha<$

৩トZ《I

$<\omega$

MUS

-IZ

2. 5 Zu

네

sonum

II) $14 Z \alpha$

जI 5

$0 r-0$

a.

It $-\infty 0$

แज $0<0$

$1-\infty$ I 0

or au

そலயく

Na

$m 0<<m$

$1-x$

ouserz?

-IUJ-

orotr

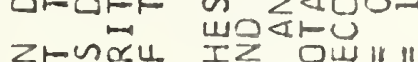

wiul

$0 I \sum$

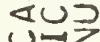

NI

車政

ar

und

oI

<5

$\Rightarrow \times$

का

ก

แบแแบ

$\sim \supset x$

10-

כa⿺n

Dx>m

zouㅣㅁ

แa

- $\propto$

一ำว

\%)

$\therefore 404$

u

$\alpha \propto$

$4>$

us 0

Durd

$1+0<2$

$12 \geqslant$

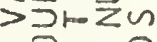

แบำ

喵

$x$

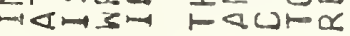

$x-$

0

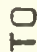

(0)

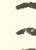

?

in

r

(1)

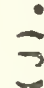

$=$

$\because$

$\propto$

No

Io

00

Y.

느-

II $\rightarrow$

70

$\rightarrow 11$

$-1-$

บu 



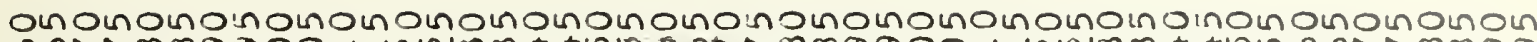

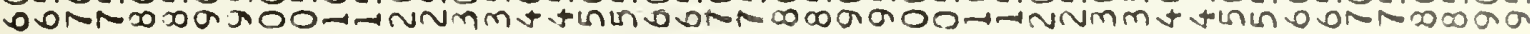

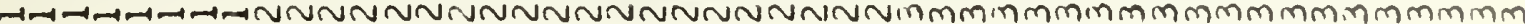

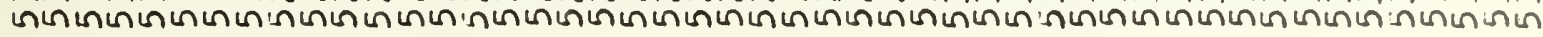

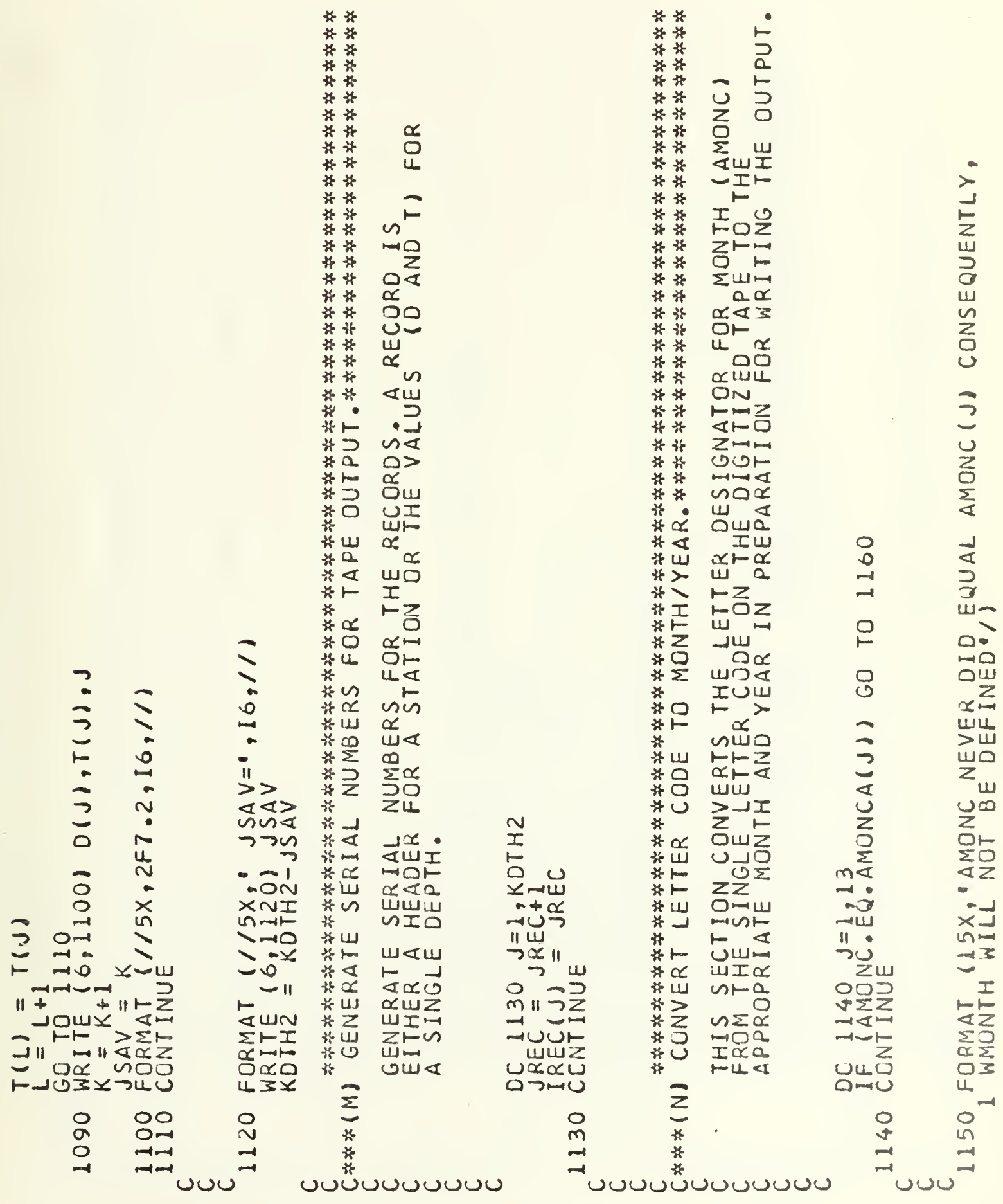





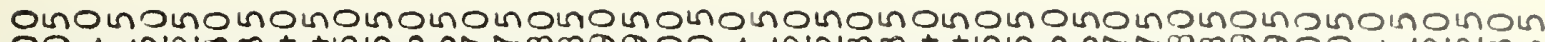

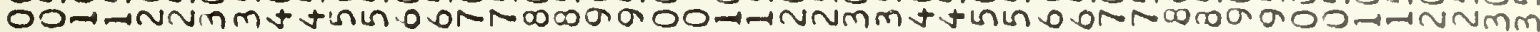

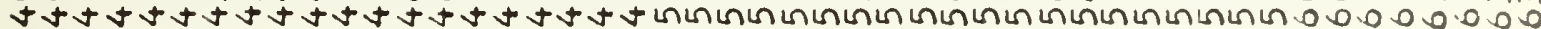

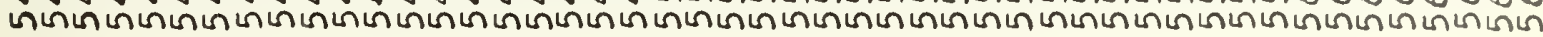

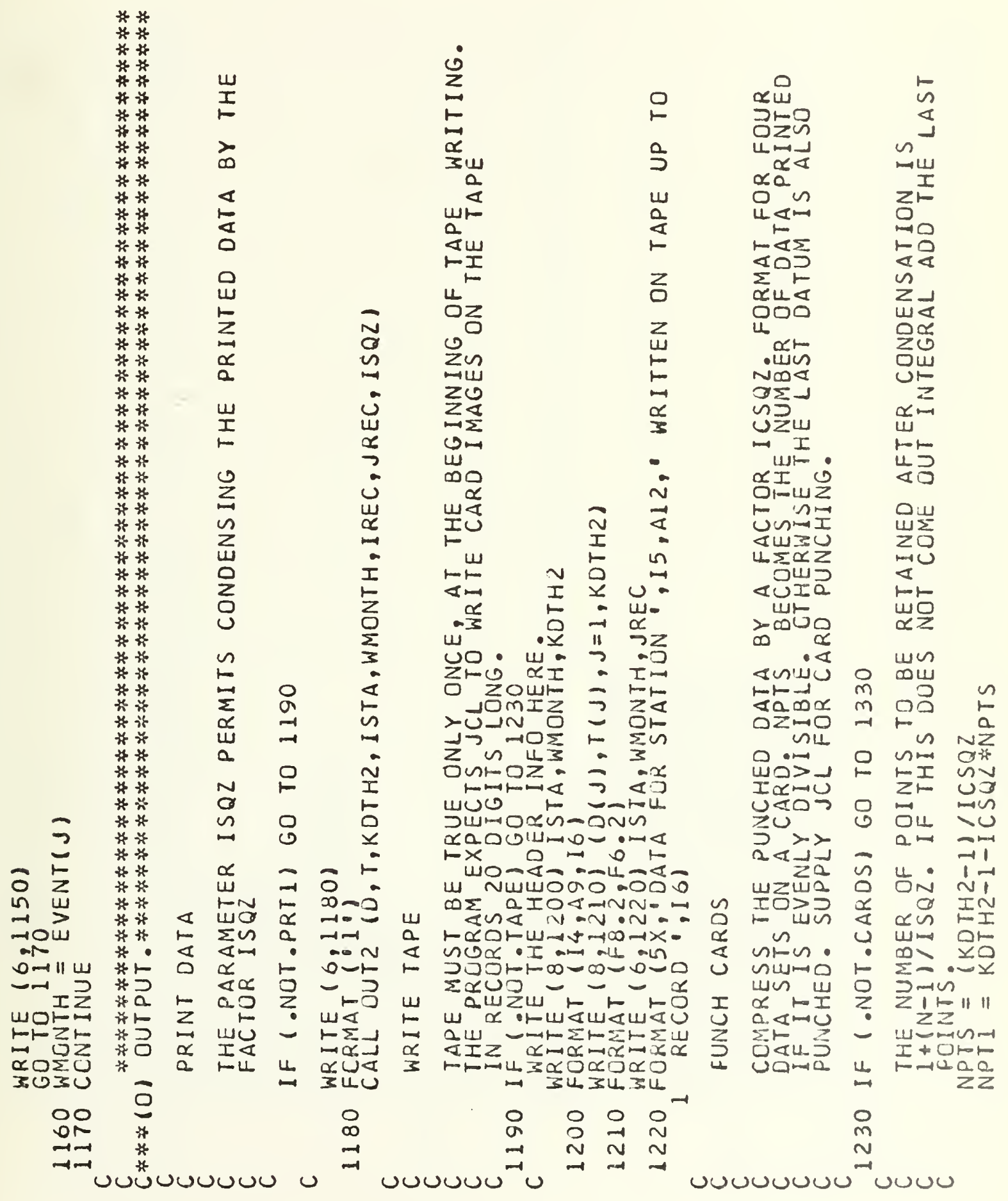





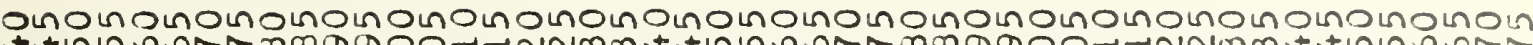

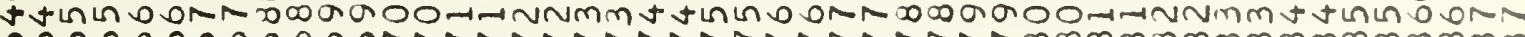
.00000000 0000-min

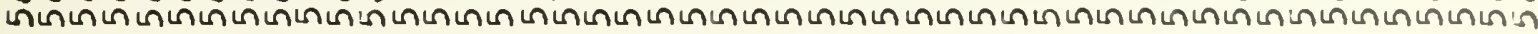

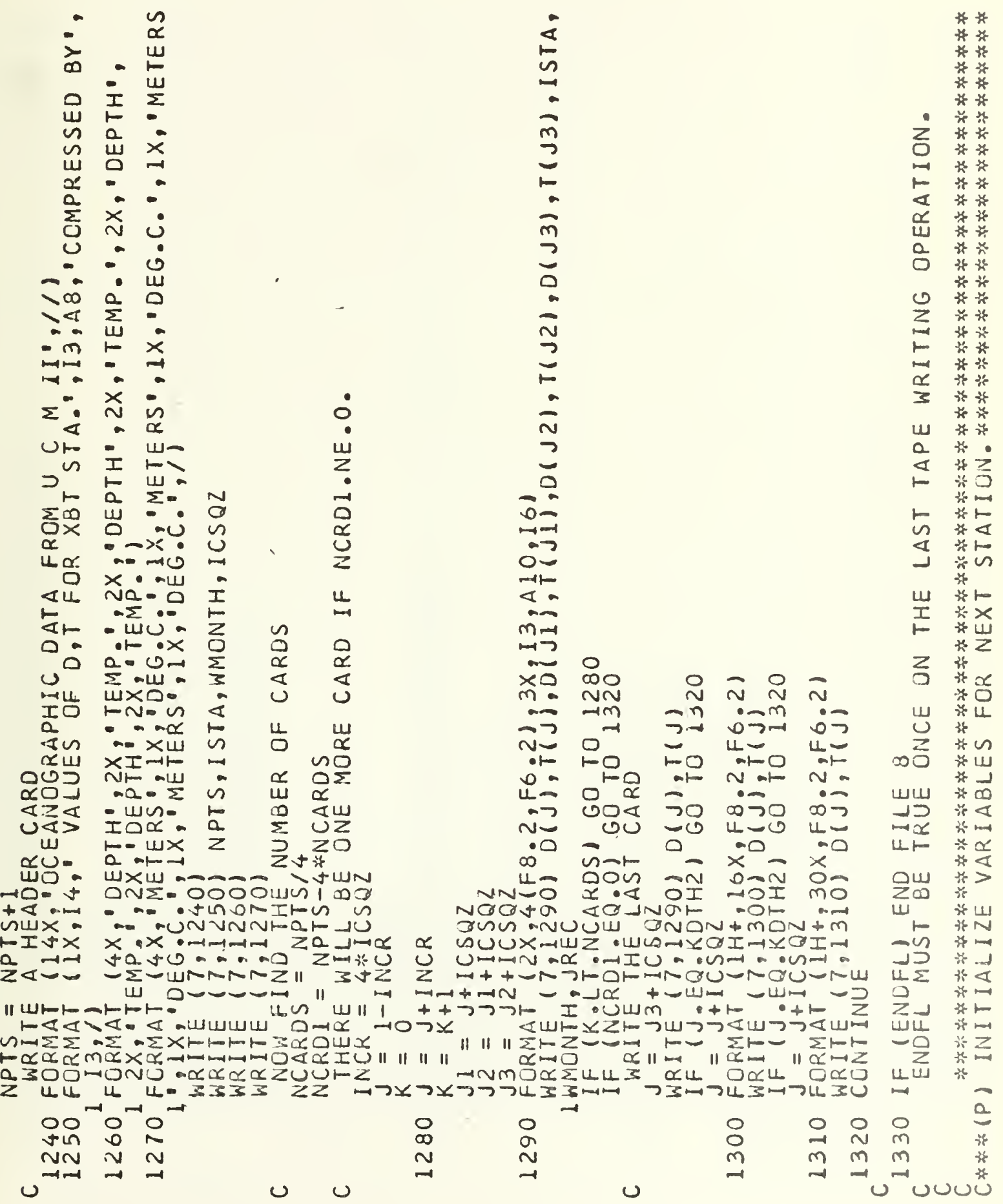




unoinino

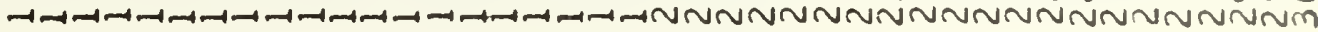

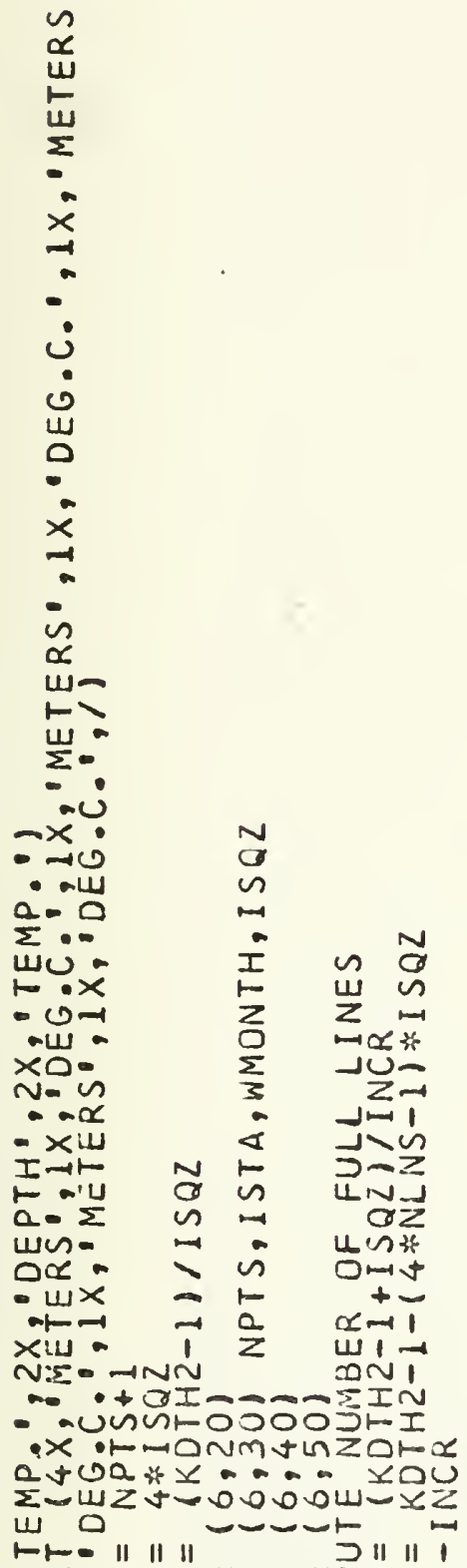

เ⺊口 \|\|$\|$

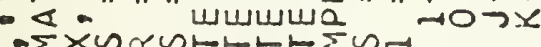

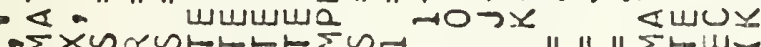

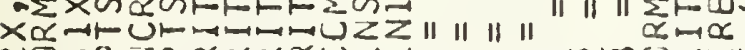

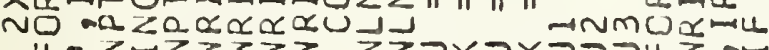

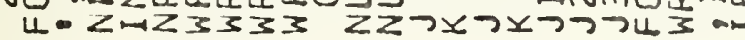
7 in

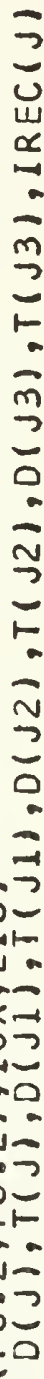

Lூ

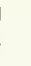

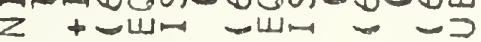
J山m

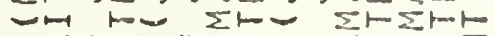

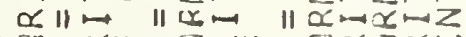

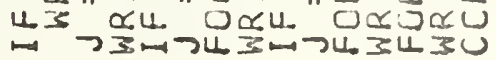
$\infty$
o OO Ond

\begin{tabular}{l}
3 \\
3 \\
\hline
\end{tabular}

in

$\stackrel{\leftrightarrow}{\alpha}$

0

$\underset{\widetilde{c}}{\Psi}$

$\underset{2}{Z}$

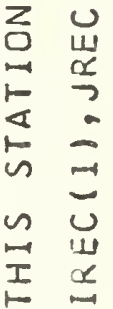

no

$\ddot{0} \dot{0} \overrightarrow{0}-\tilde{0}$

ơx mas

U $\rightarrow I-1$

W>-mat.

$x=0-\infty$ ⺊ Z<.

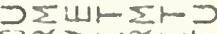
Uบ

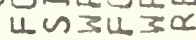
$0_{n}^{-1} \stackrel{0}{-1}$ 



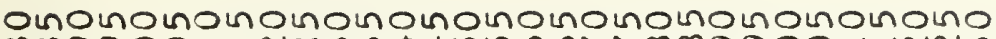

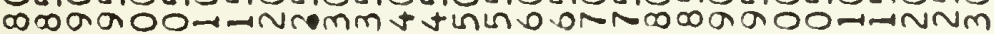

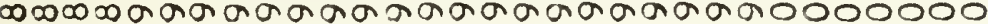
unininininininininininininininininininininininin 0000000 unounounounomono

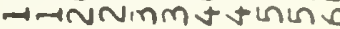

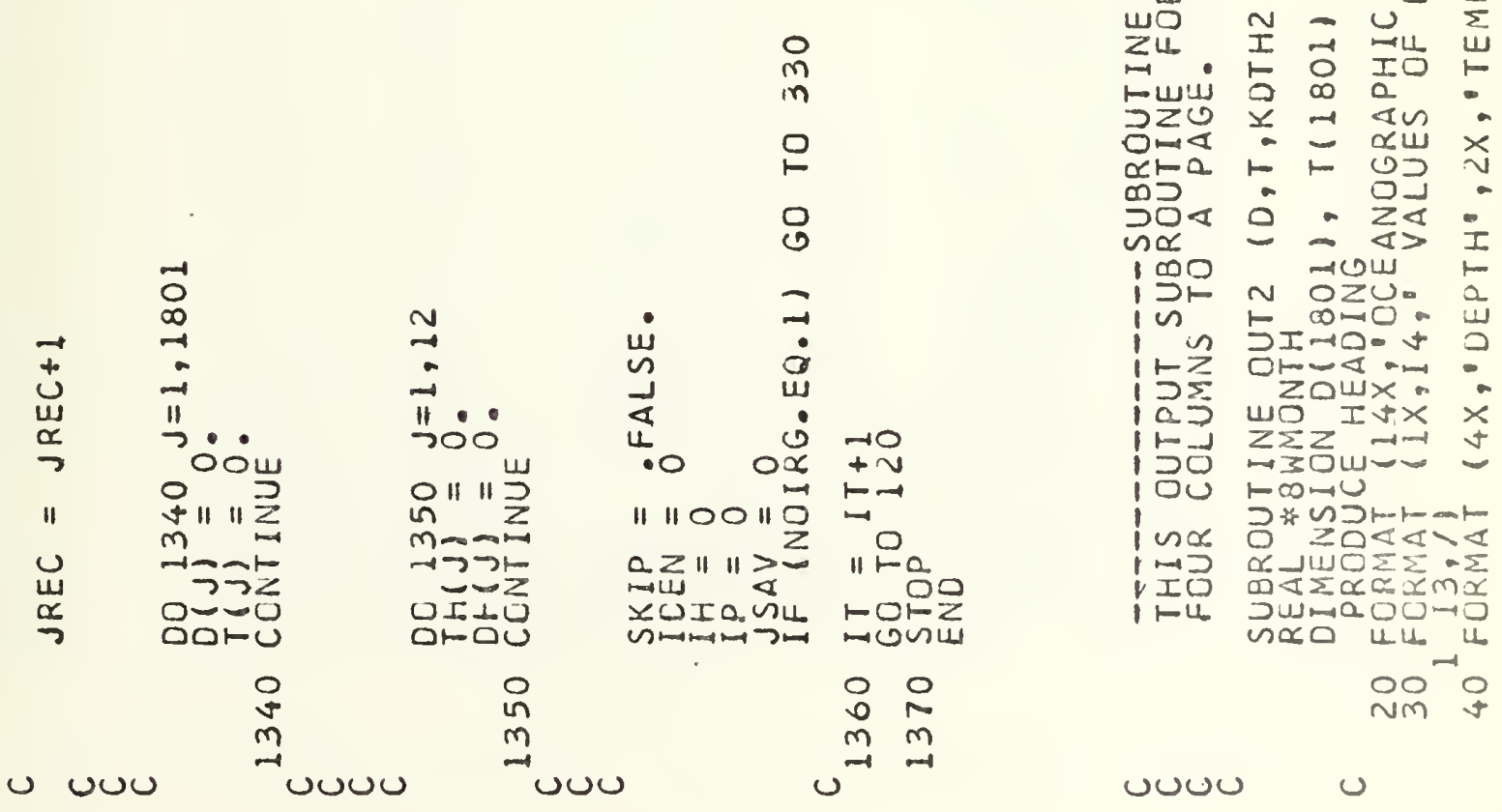





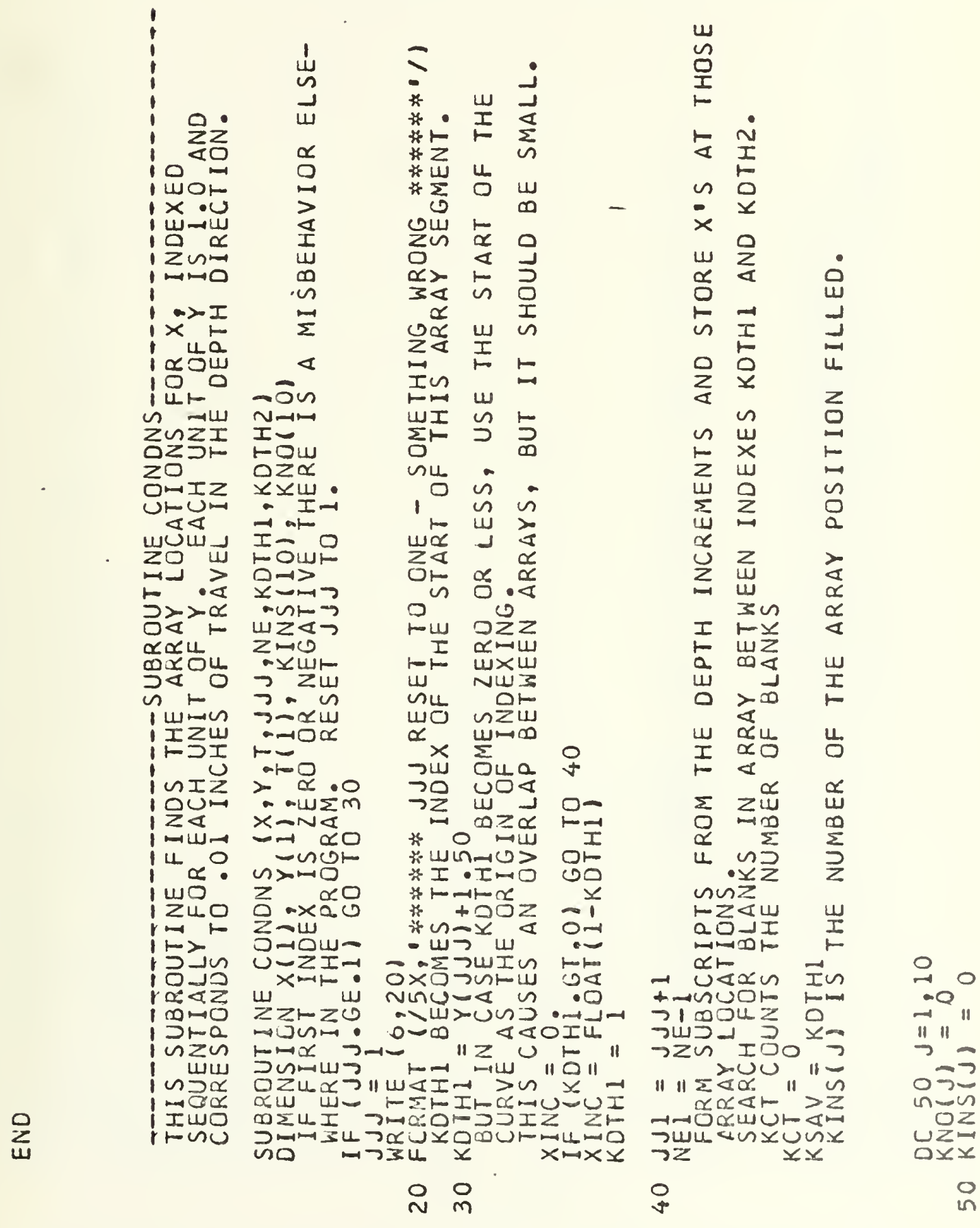





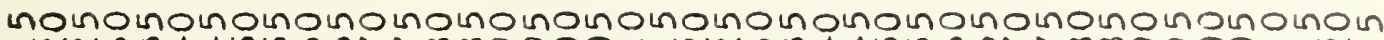

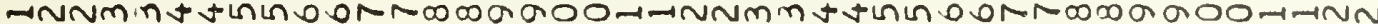

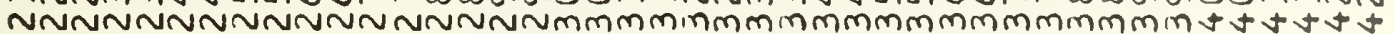

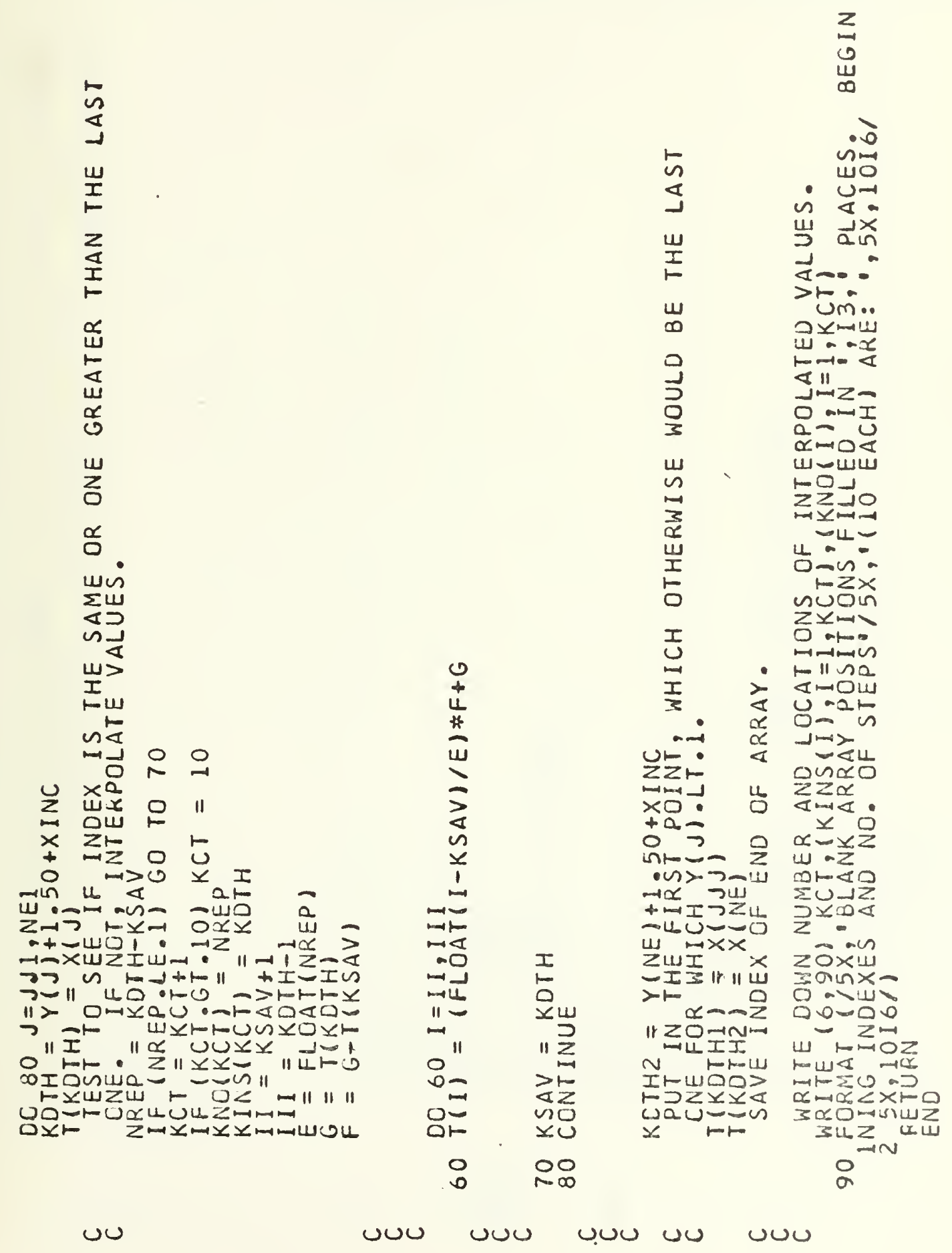





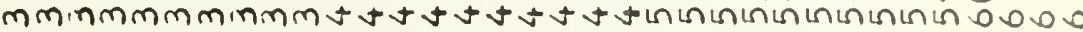

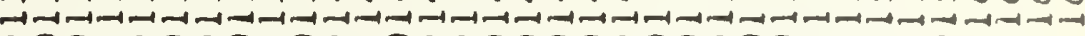
0000000000000000000000000200000002

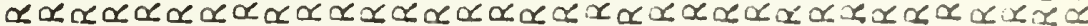

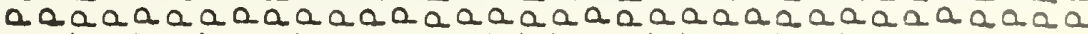

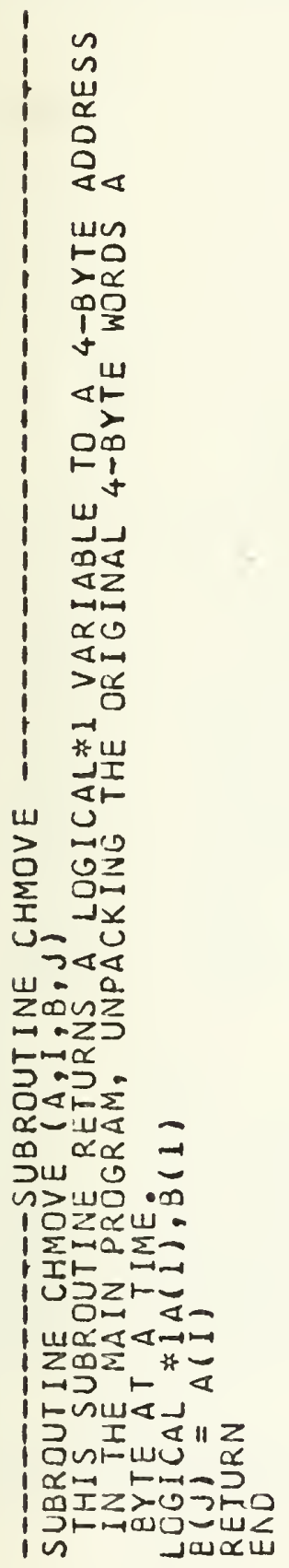

$\circlearrowleft \circlearrowleft$

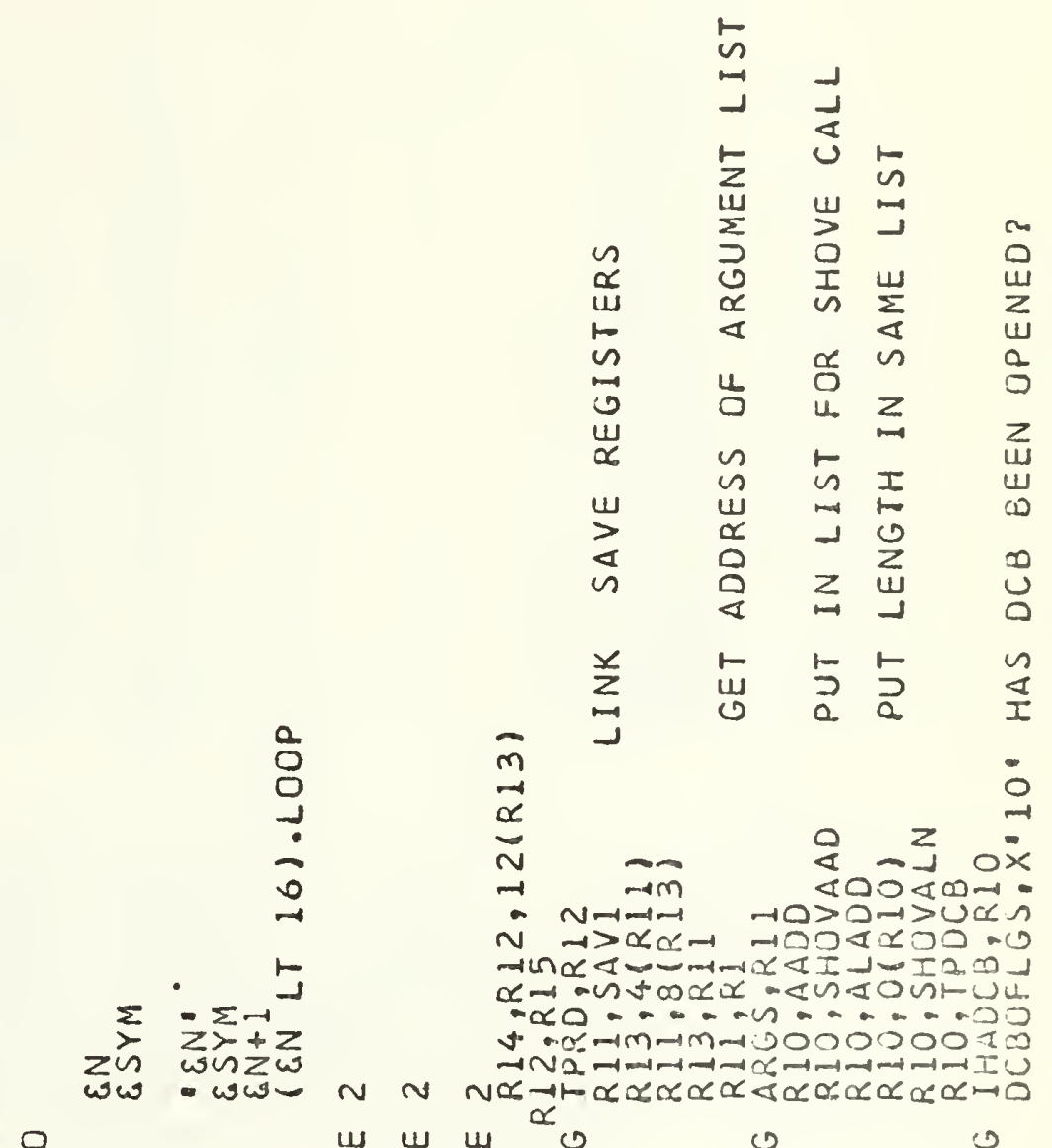

○ ш ш ш

ज以 $\sum_{m=1}^{Z}$ Z

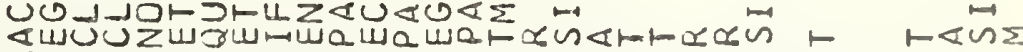

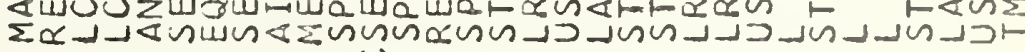
$\omega$

$\begin{array}{ll}0 \leq \sum & a \\ 0>\omega & a \\ \sin z & a \\ 0 \omega \alpha \omega & 1\end{array}$



000000000000000000000000000002000000000000000000

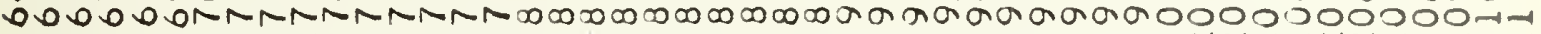

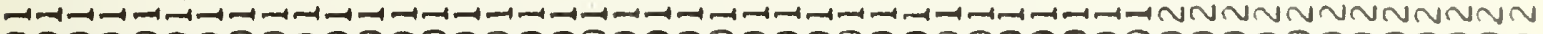

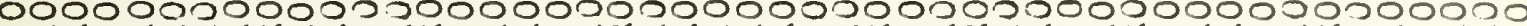

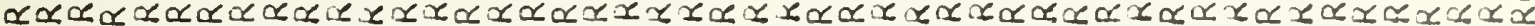

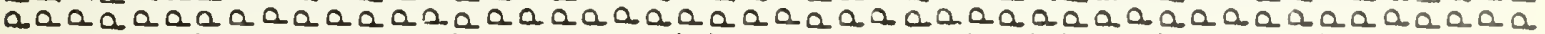

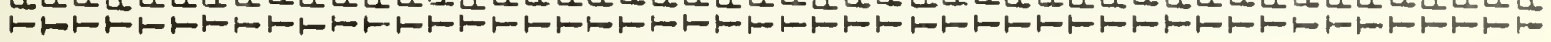

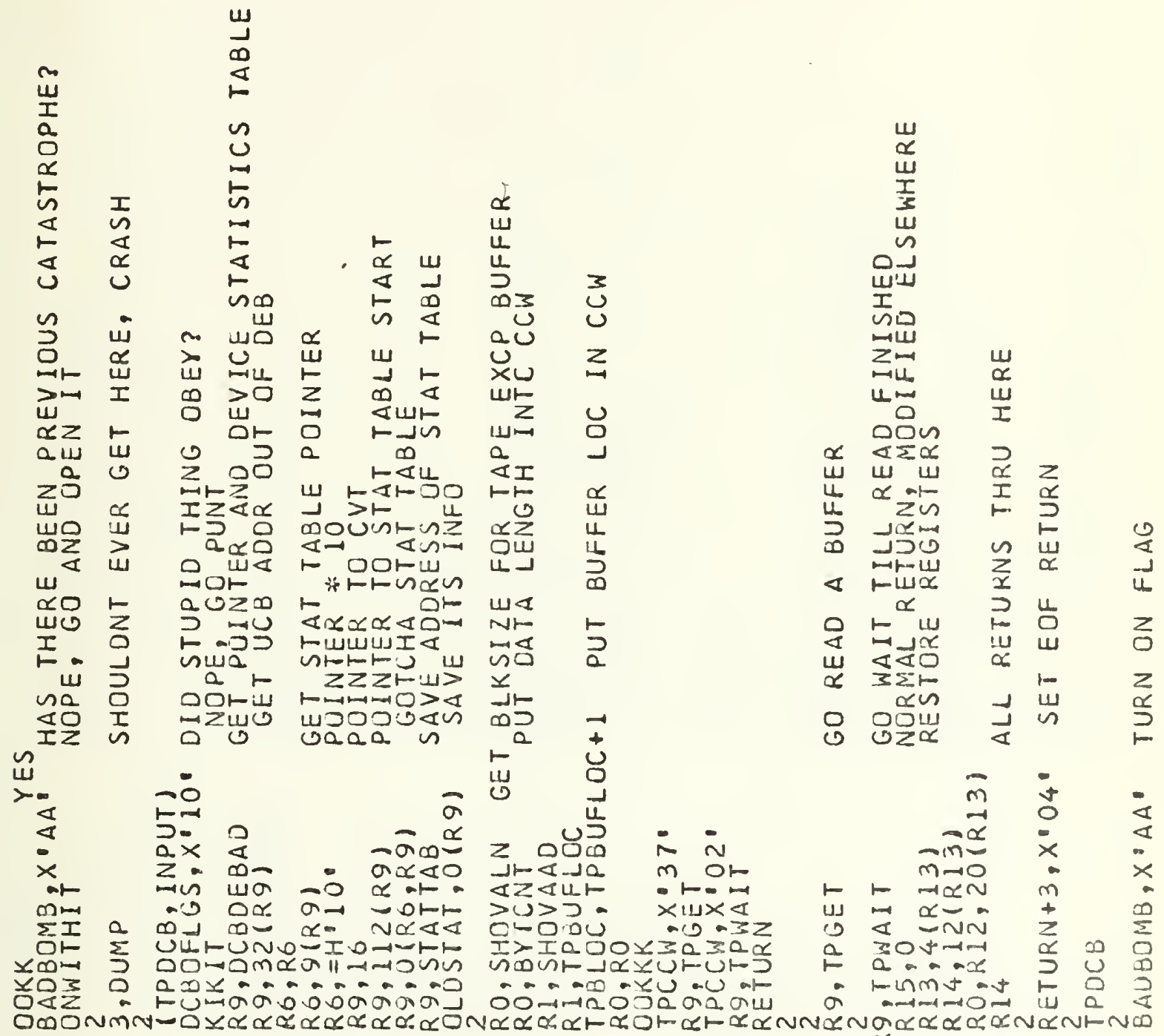

แоแ

UะטZ

$0<4<4$

OEZ

Z

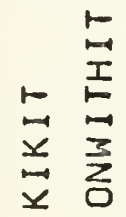

$\frac{x}{x}$

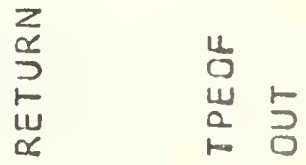



000000000000000000000000000000000000000000000000

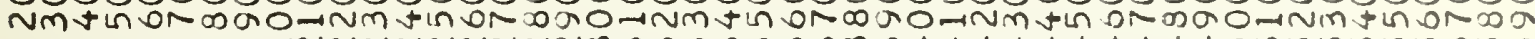

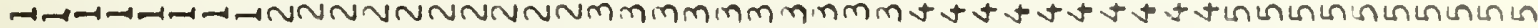

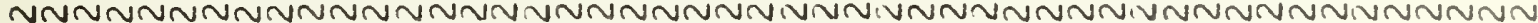
000000000000000000000000000000000000000000000000

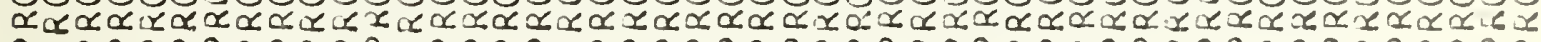

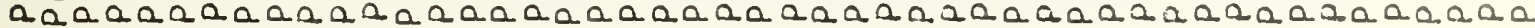

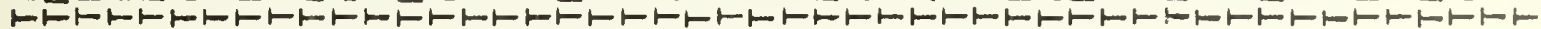

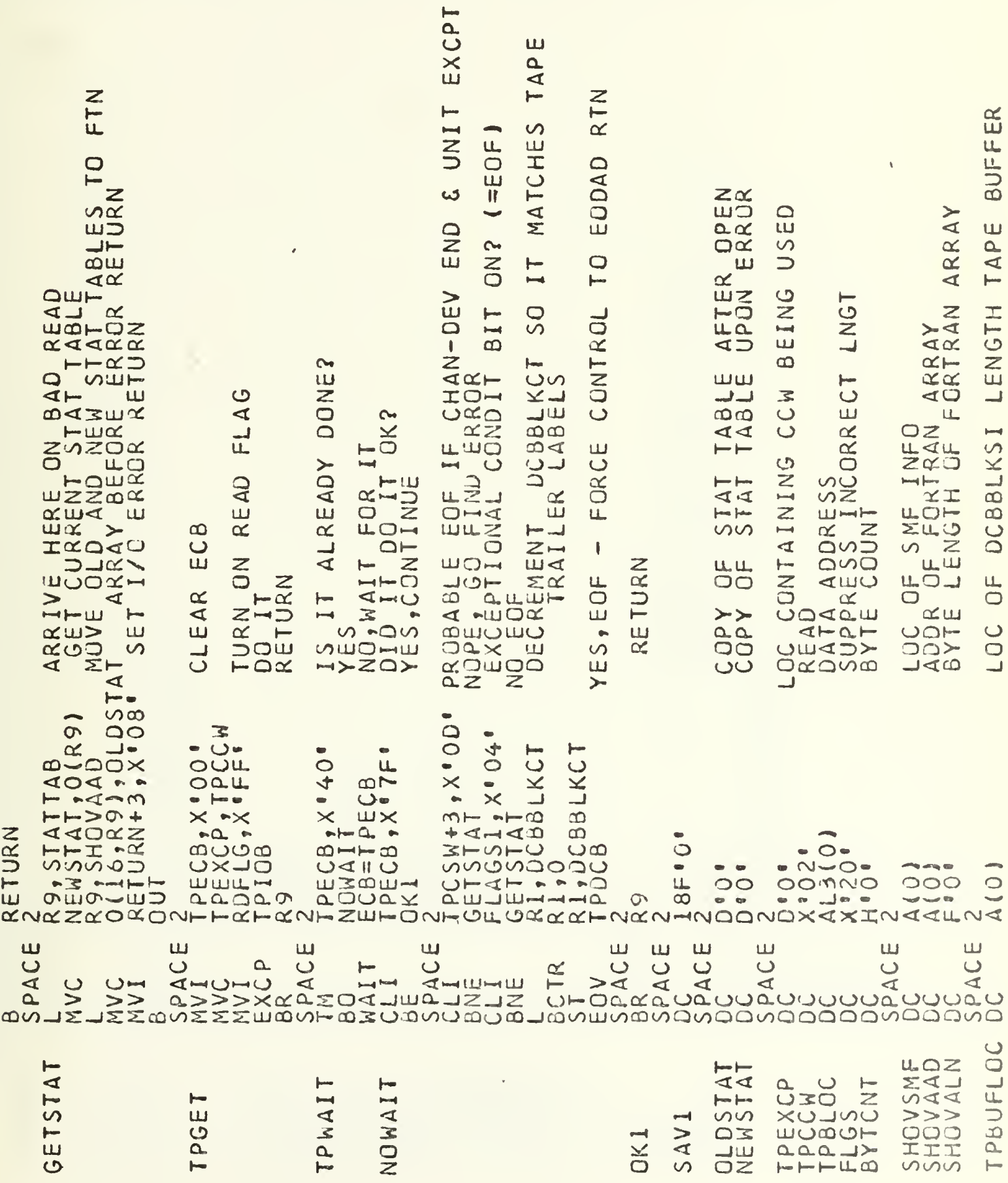



000000000000000000000000000000000000000000

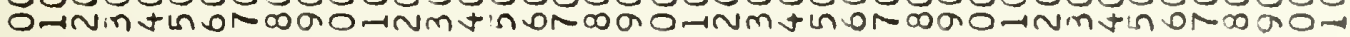

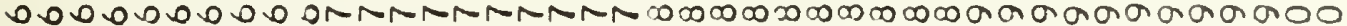

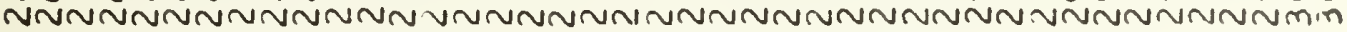

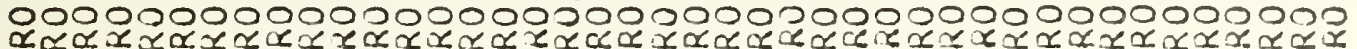

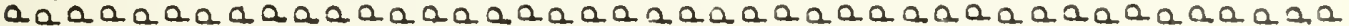

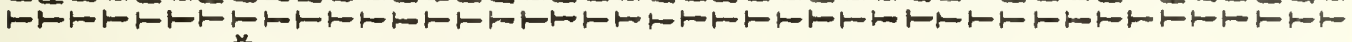
*

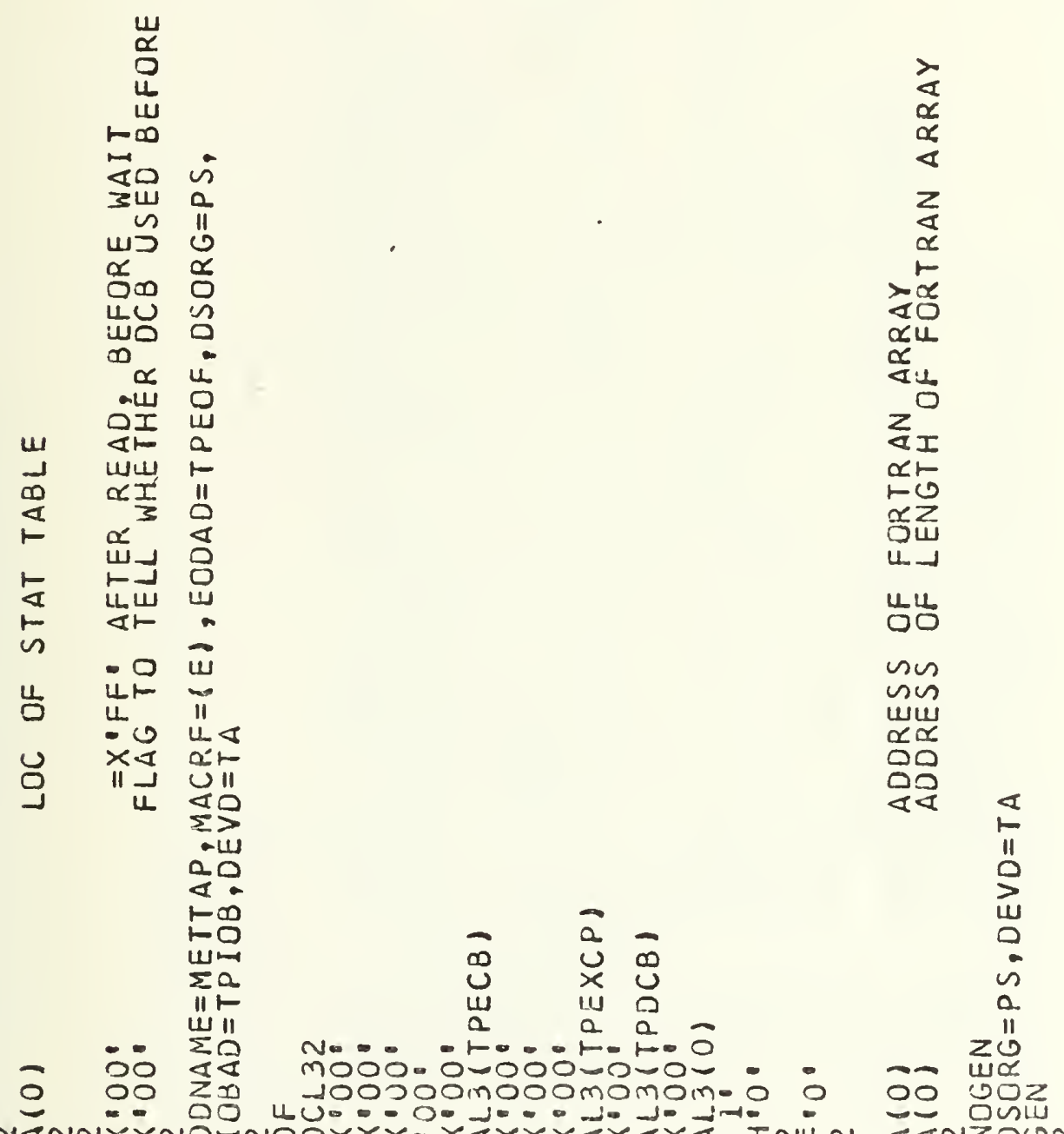

NanNix
山 யس
u
U) U1
$\circlearrowleft$ Uu
Uю $\vdash ய \circlearrowleft$
$\omega \geq 0 Z \omega+\infty$ $\alpha \omega \infty$ a 1000
nomusous

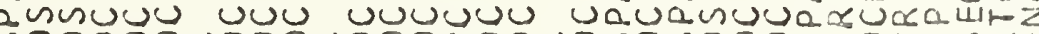

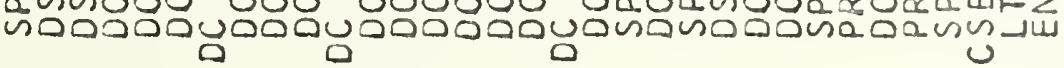

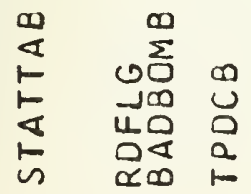

wo $\propto \circ \propto \vdash$

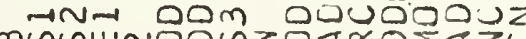

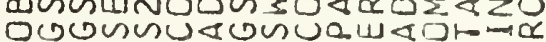

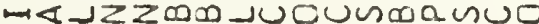

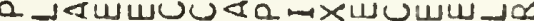

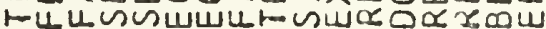

$\bigcup_{\sim}^{\infty}$ no

2 $\quad 0<\frac{1}{4}$ $\frac{0}{a}$ 

u.

$\times$

○

z

U

a

a

$<$

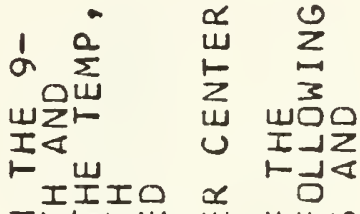

ZIIIO \&

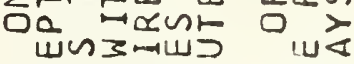

zoz vina

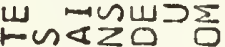

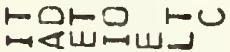

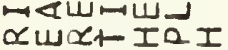

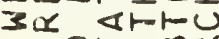

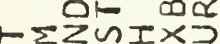

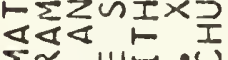

$\sum \alpha$ w

\& 0 - n 30

00س음

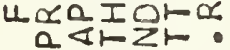

$>I \frac{W}{\alpha}$

ZI $\alpha$

낭 $\frac{\alpha}{4}$

这r

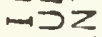

$\alpha 1<$

UZト

nबa

แบ๊

0 은

w它

$<Z=$

$\alpha-u$

U 1 <O?

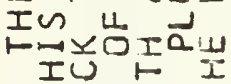

IトU⿺𠃊ே

두에

DつI

แD口

$\propto$

$>0 u$

\&u

$3 \vdash 1 \sum^{\infty} 00^{\Sigma}$

व 2

sम 1 क 00 $\rightarrow I m$

山x Zய 4.J上ト

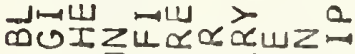
कuIZu เO

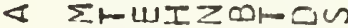

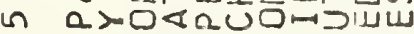

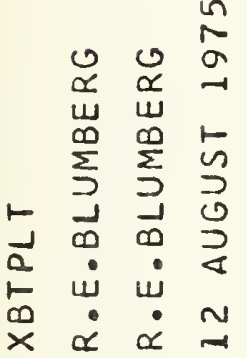

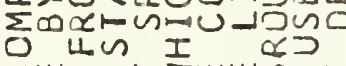

गए

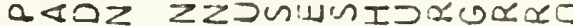

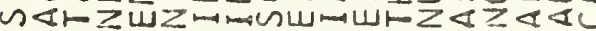

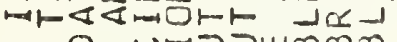

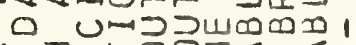

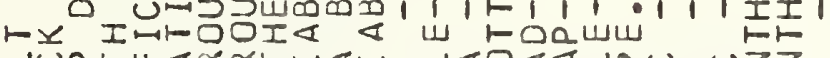

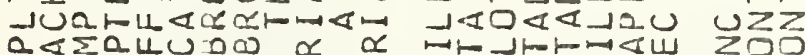

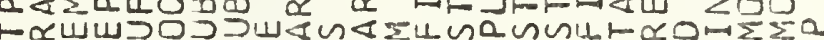

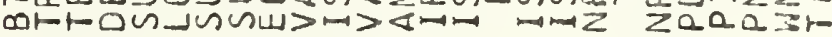

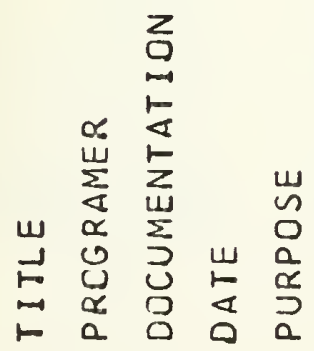
(1) $0 \operatorname{lin} Z \propto \propto \propto ⿻ 上 丨$ 0 $\sum \vdash 0$ in ZOL IIA wa I $上 \Sigma \sum$

는 $\backsim \sum \ddot{x}$

トエய」$x$

$$
\sum_{0}^{2}
$$

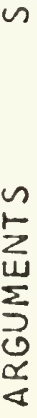

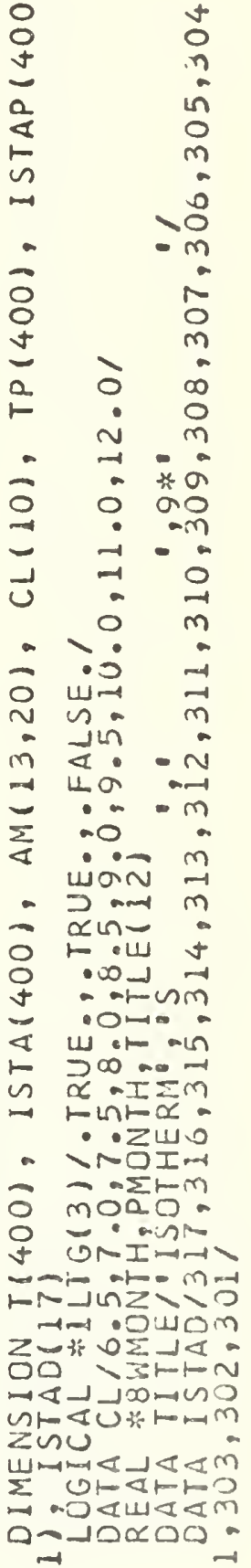

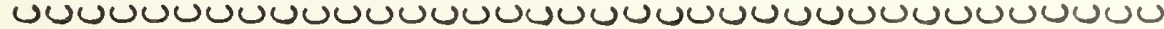





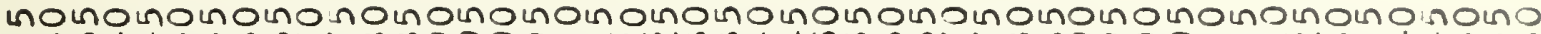

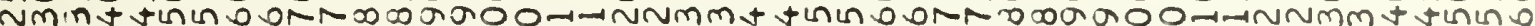

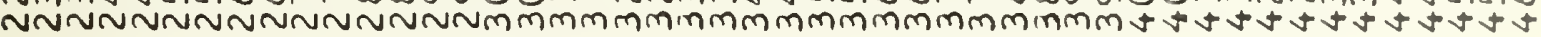

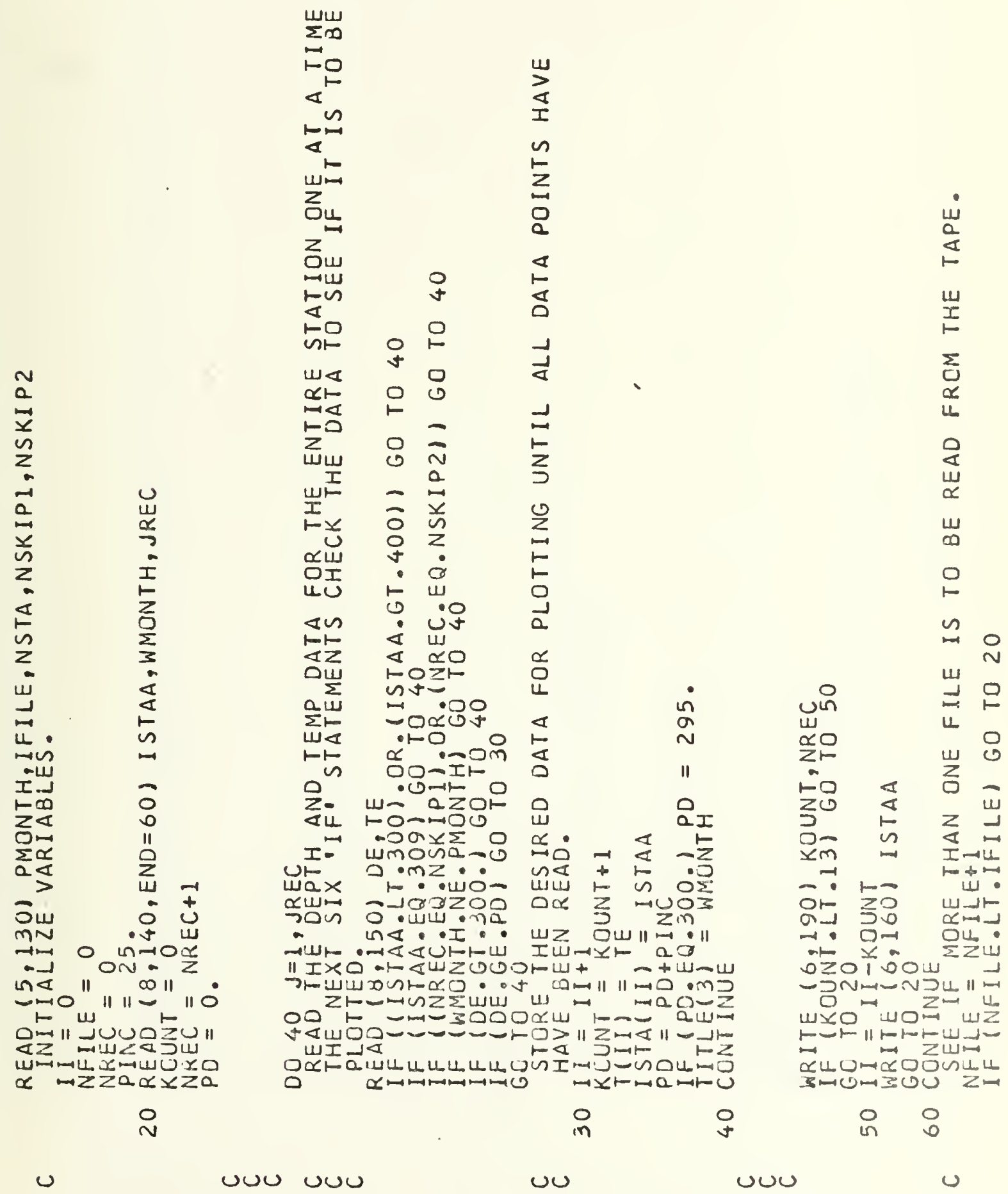





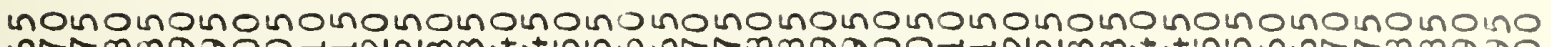

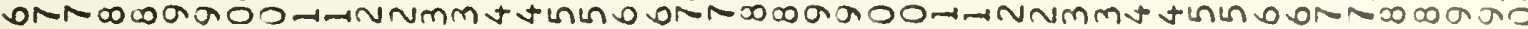

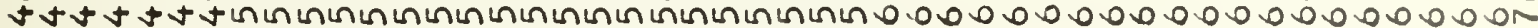

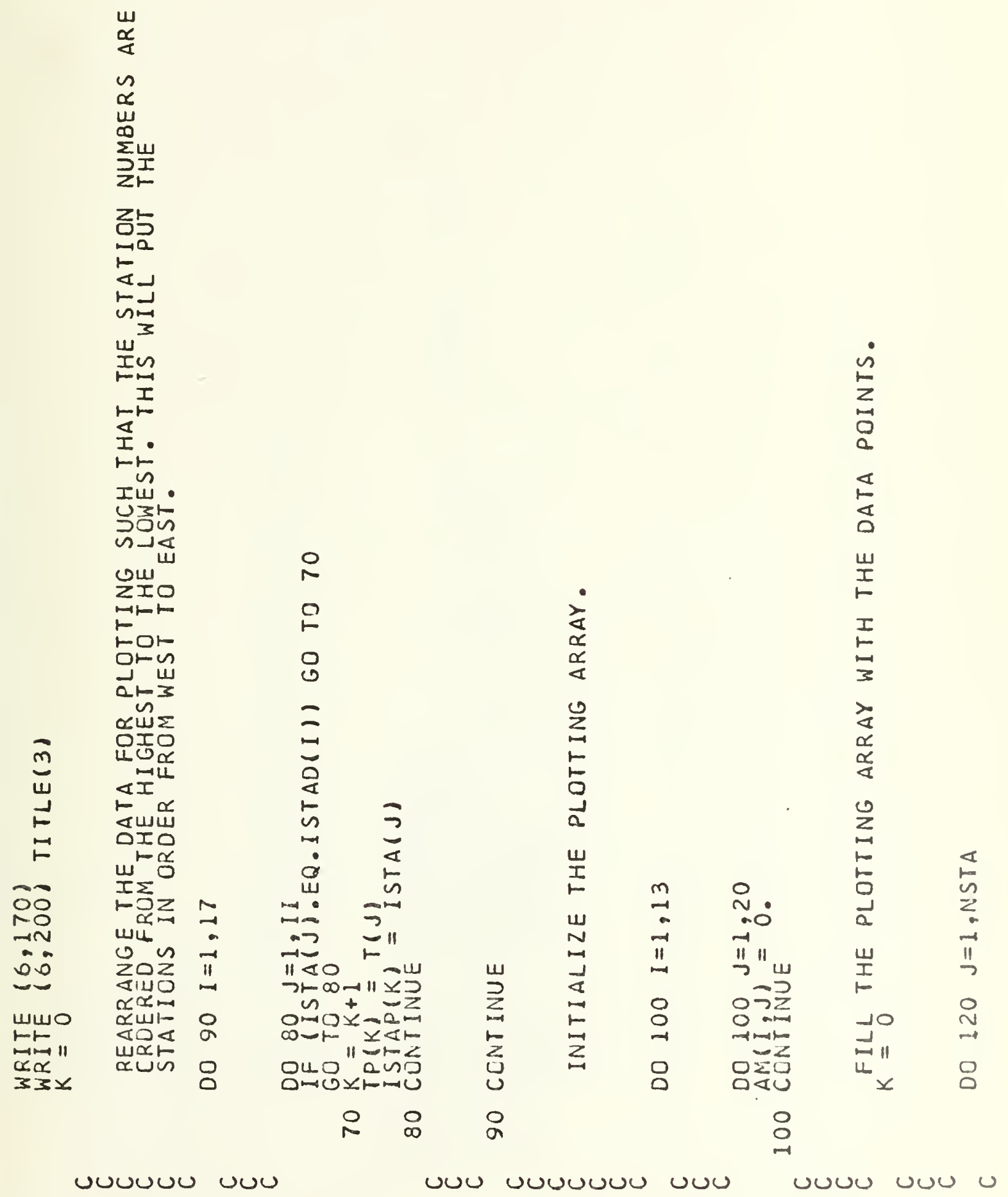





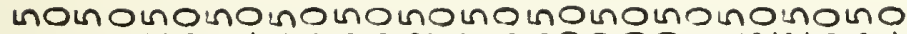

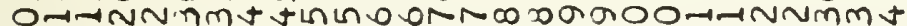

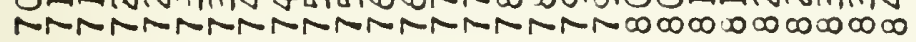

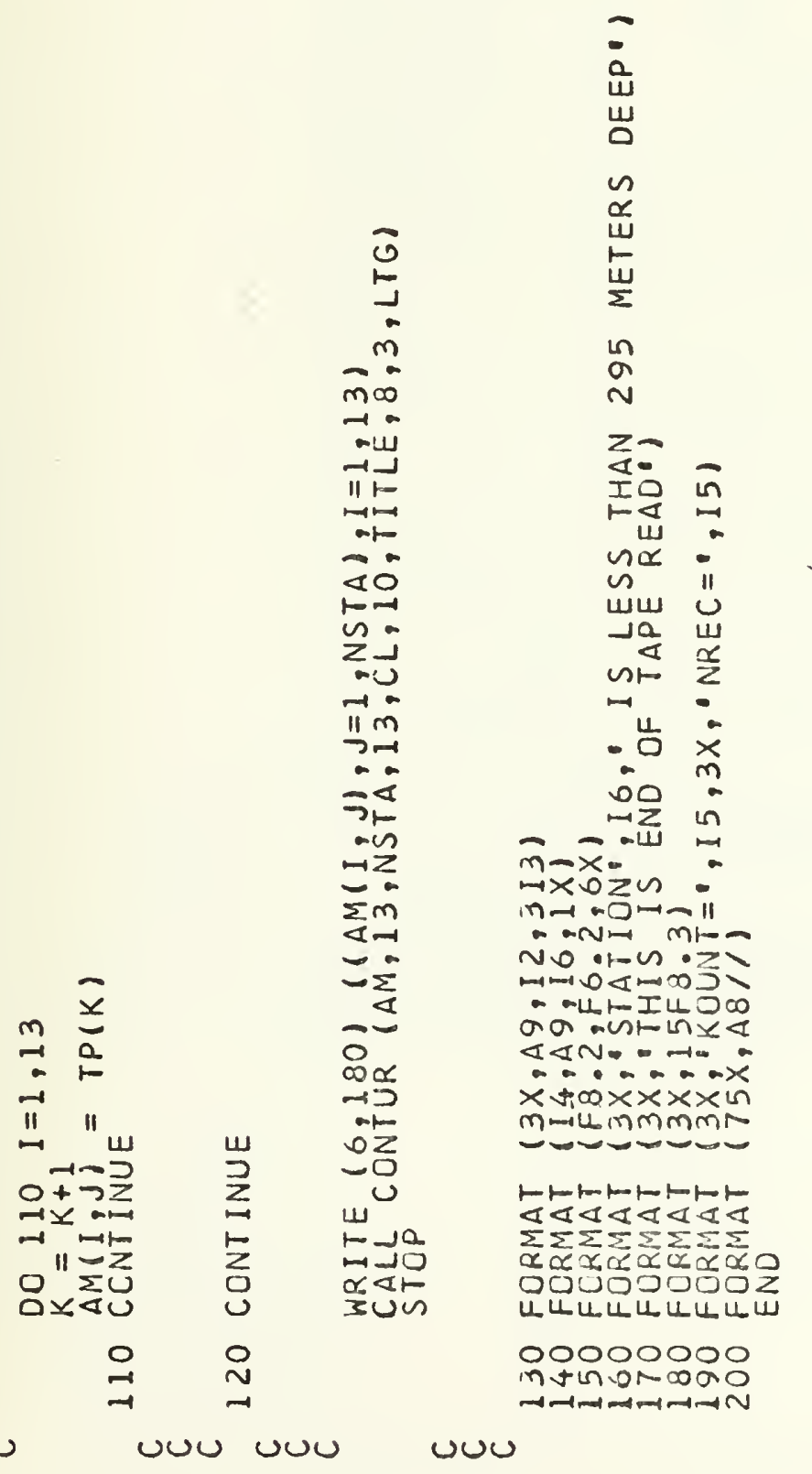





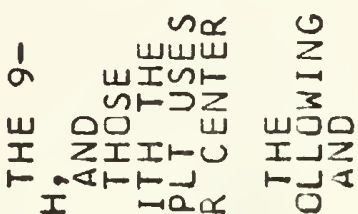

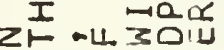

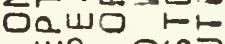

2๐ั

$\sum_{11} 0<\alpha \geq \frac{2}{1}$

Fn

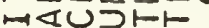

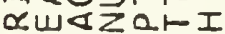

ऽ๘ם トZつ-

$1 \sum_{1}^{1} 000$

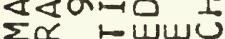

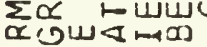

OOIFU.

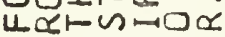

${ }^{a}$ इOU $\dot{0}$

II) $\Sigma Q W 3$

Find

IU

IF

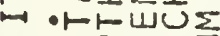

$30<0,1 \rightarrow 0$

เดயUエฯ

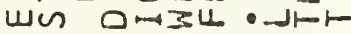

गॄर

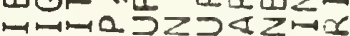

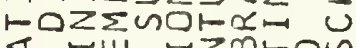

a $\varangle$ wU

$\varangle$

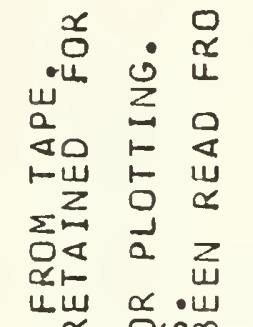

ons us

$u \propto$

뚀 шس

गயZ जm>

$F \alpha<$

owo

$\cup D \&$

Zน

$z_{n=1}$

u

oDa

แயய

서

nड

>ய之

¿०

싱

दा山以

dro

ज口

zun

Fu

$10 u$

o

口U> > $\infty \varangle \quad \varangle<z$

2红I

$=2$

II

I>U ल

เరำ

Nin

WI

Ir n

मnய

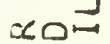

யயน

Q⿻日土龰

$0<0$

गर्ष

Doxa

山冈

ax

u $?$

วด

Uᄄ口

ठㄴ

\&

ต

บu

U⺊

xz $\dot{x} x \bar{a}$

$7<4$

느 سயإ

«之.

Din $0<1$

는 IIQ

20 III

d

แล

I $1 \sum \Sigma$

는

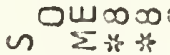

เIய山”.

ZZF以过 วดงแนx

Oயz $\alpha \propto x$

ט०m

$$
\text { טய I }
$$

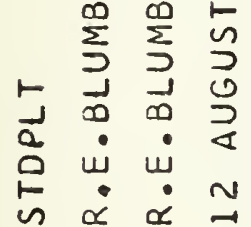

n<之 n

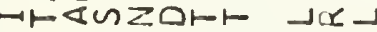
乙0யग

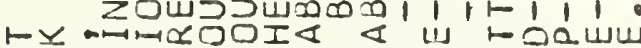

$1,1 \frac{1}{1} 1$

गQ

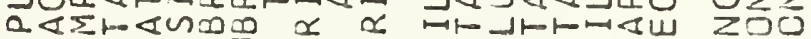

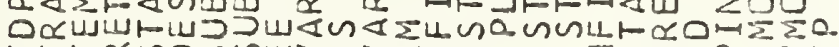

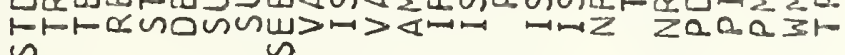

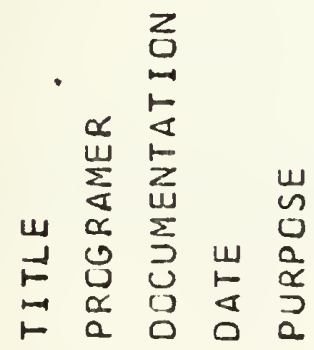

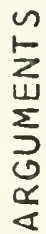

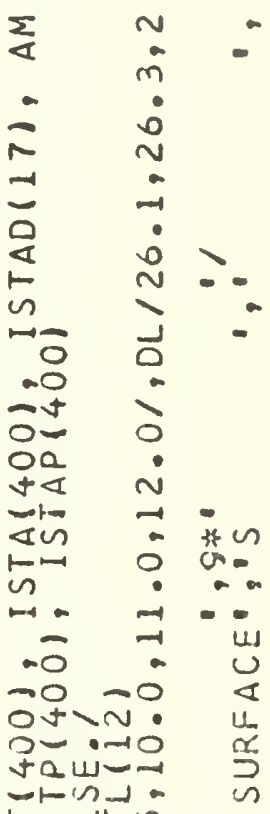

$\vdash J \boxminus$ ก

- Im

$\rightarrow 41-0$

$-n \cdot m \cdot$

$0-\infty-\infty$

OA. - .

touna

- $2 m+$

a. $-\vec{x}-n$

z-tw.

$00 \cdot-100$

म-1 in

$n$ - into

$\sim \supset-\infty-$

$\rightarrow \vec{x} I+\Sigma \cdot$

O m-Fin $x z$

$0-\cdot z \cdot$ w

tolON IF

$-N-\sum a n$

- má oZ

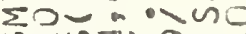

जNOINGmu

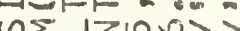

$x \rightarrow 0$. NW $z^{2} * \sum 0$ O. I- Nトー

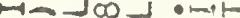

no<: 0 O Znu $v$

w $\rightarrow \Delta \rightarrow<4$

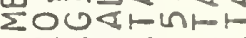

HNUWG 01

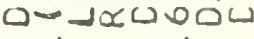

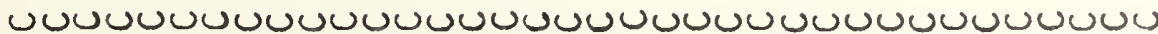





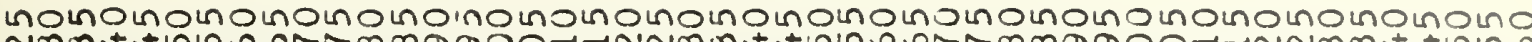

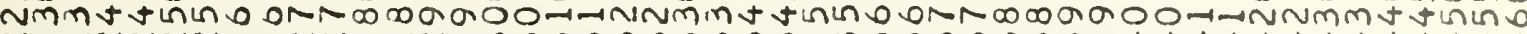

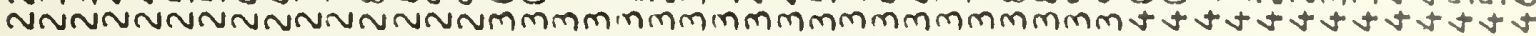

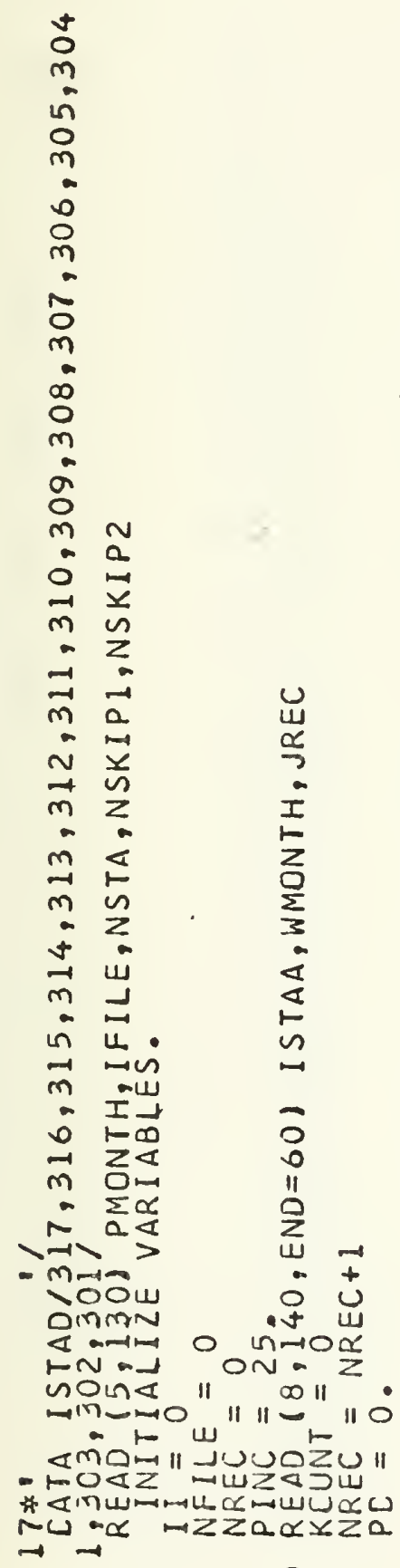

ㄱ

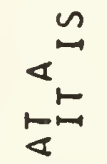

w"

z

Ou

zu

On

トレ

$\stackrel{1}{1}$

us

แธ

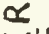

프미

Zト

w

世芩

Iㅍ

-I

危

告的

난

षा

$\vdash \sum$

$\varangle$

or

$>$

inis

(1)

-4

$\leftarrow=$

$\sum_{0}$

जx

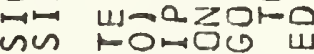

- Ox: D的

n-

แ-แ山

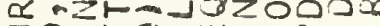

$\rightarrow 0$-0.4 $\cdot$ Lna

- uña I

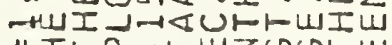

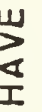

$\stackrel{n}{\llcorner}$

용

$\leftarrow$

吕

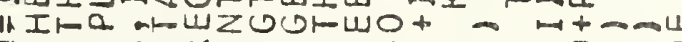

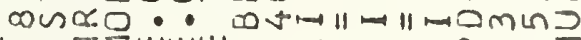

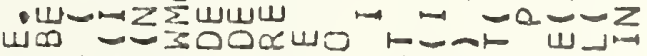

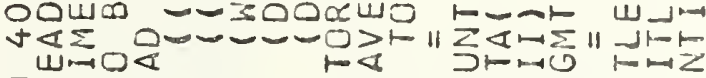

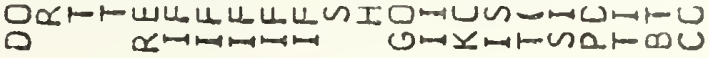

우

o

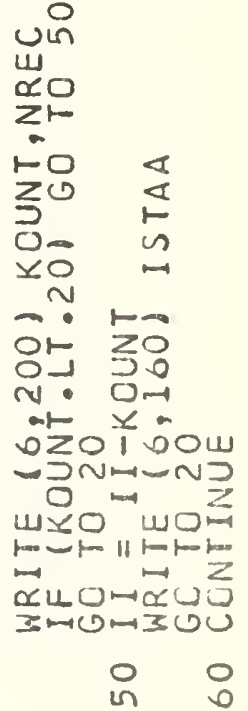

us 



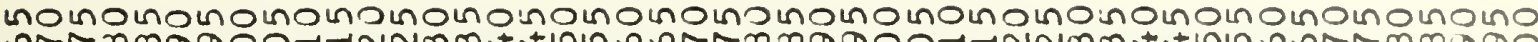

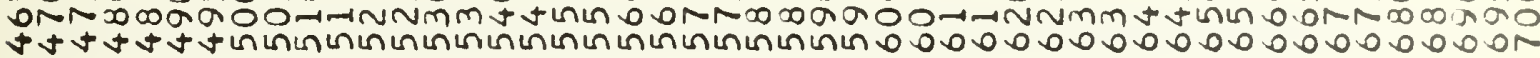

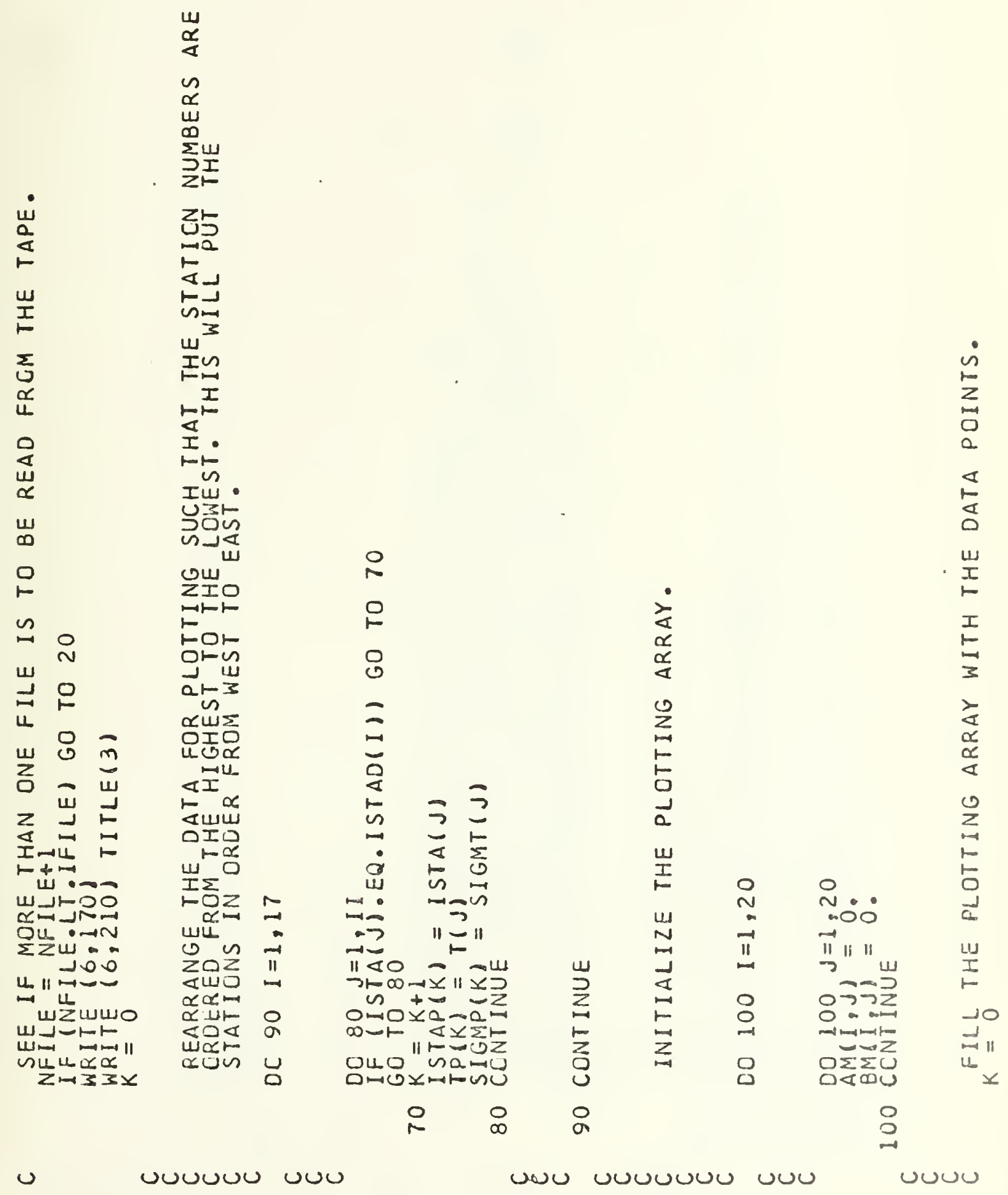





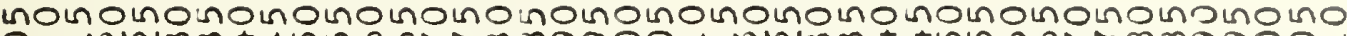

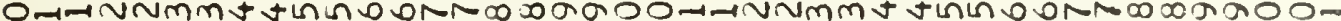

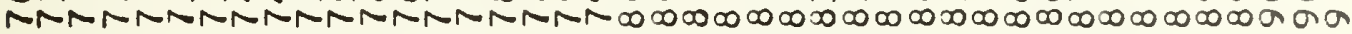

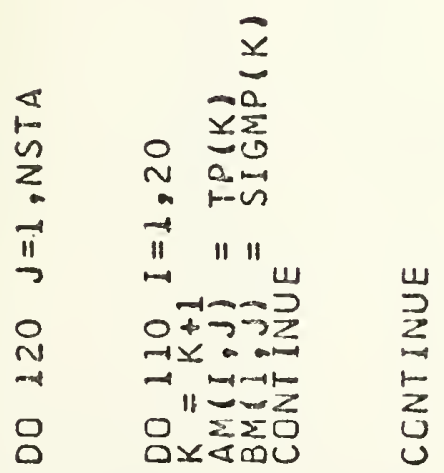

$\stackrel{0}{\rightarrow} \stackrel{0}{=}$
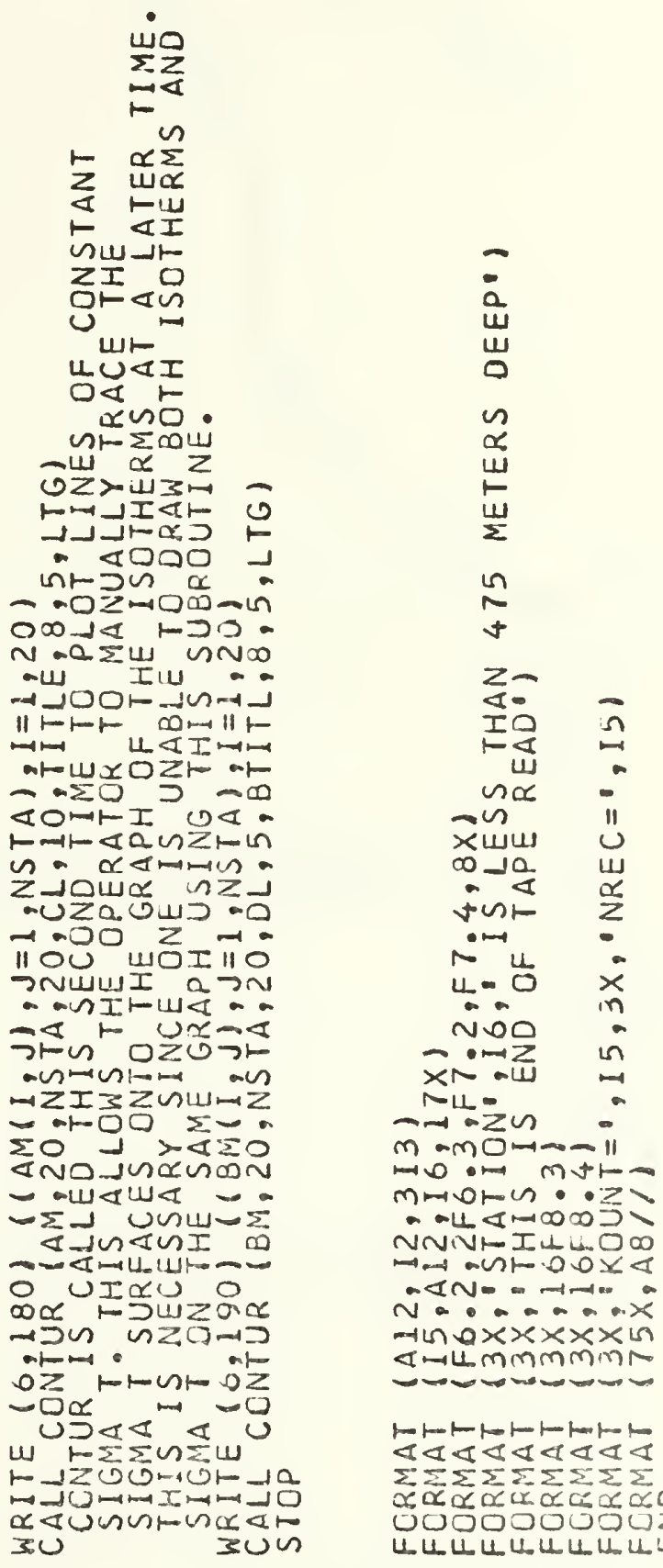

トトトトトトトト $\varangle<4<<<4<4$ $\sum \sum \sum \sum \sum \sum \sum \sum \Sigma$ $\propto \propto ⿻ 上 丨$

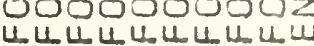

000000000 min OND DOO-

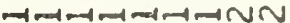



APPENDIX H

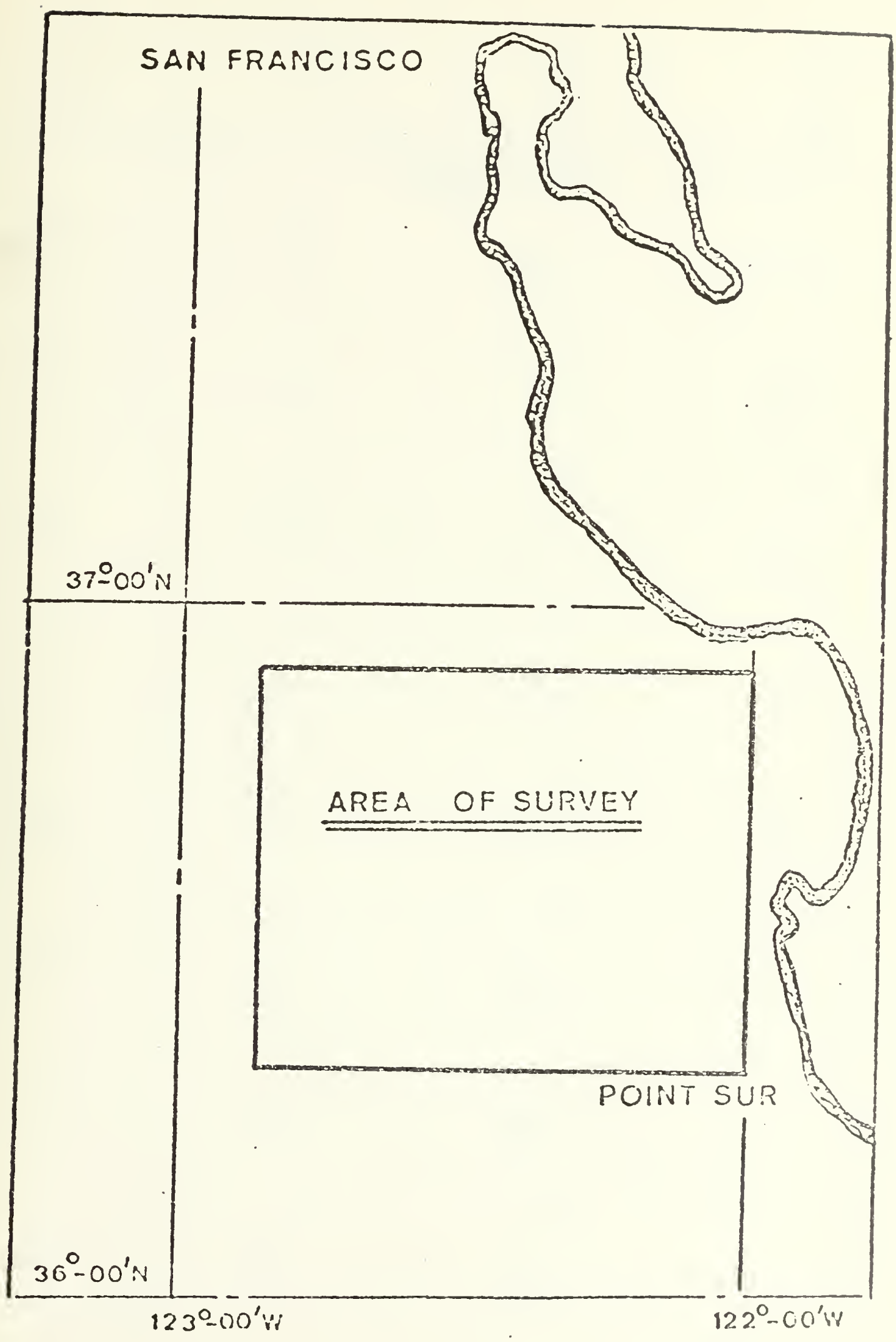

F1g. 1 Area of Survey. 



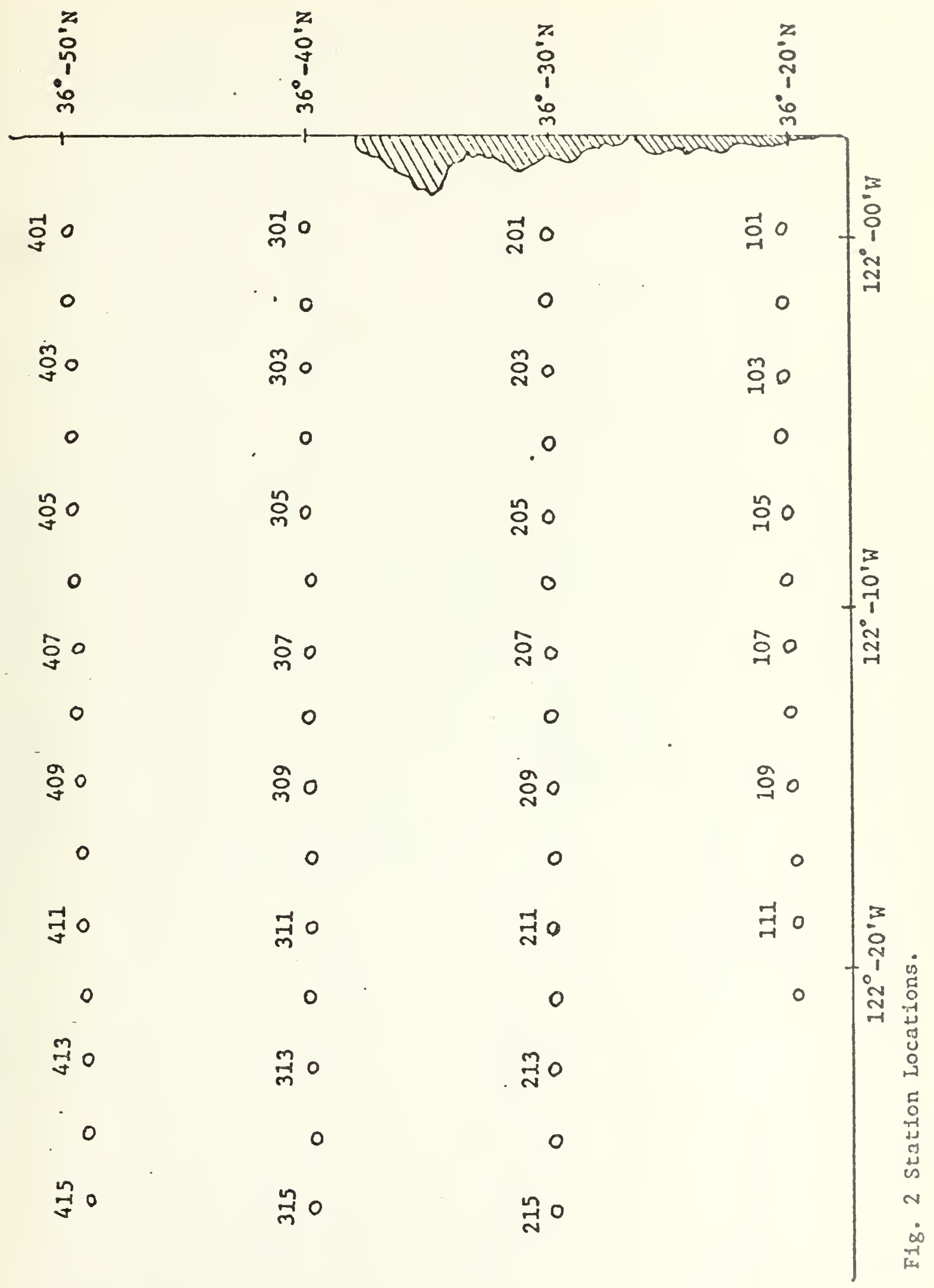





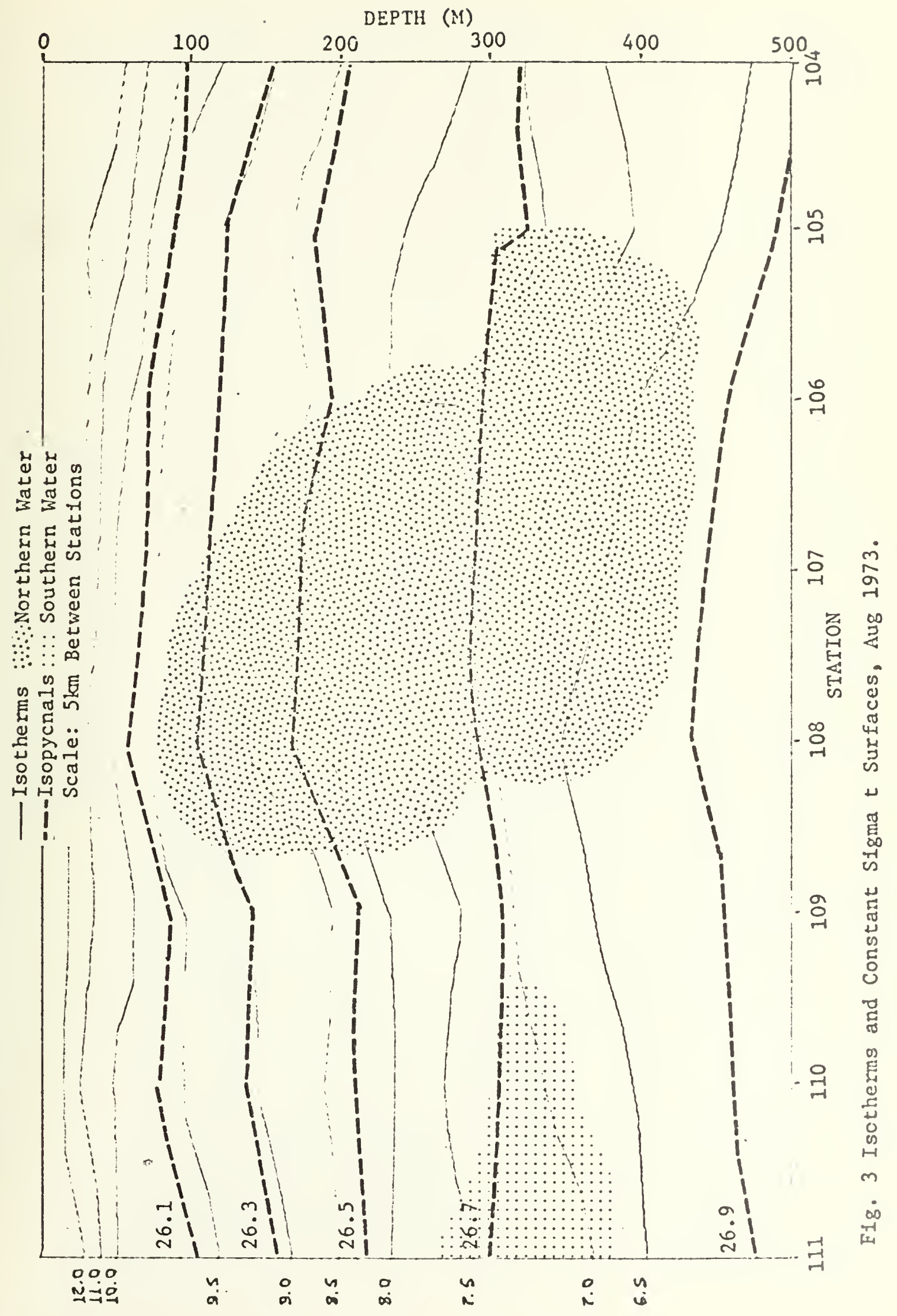





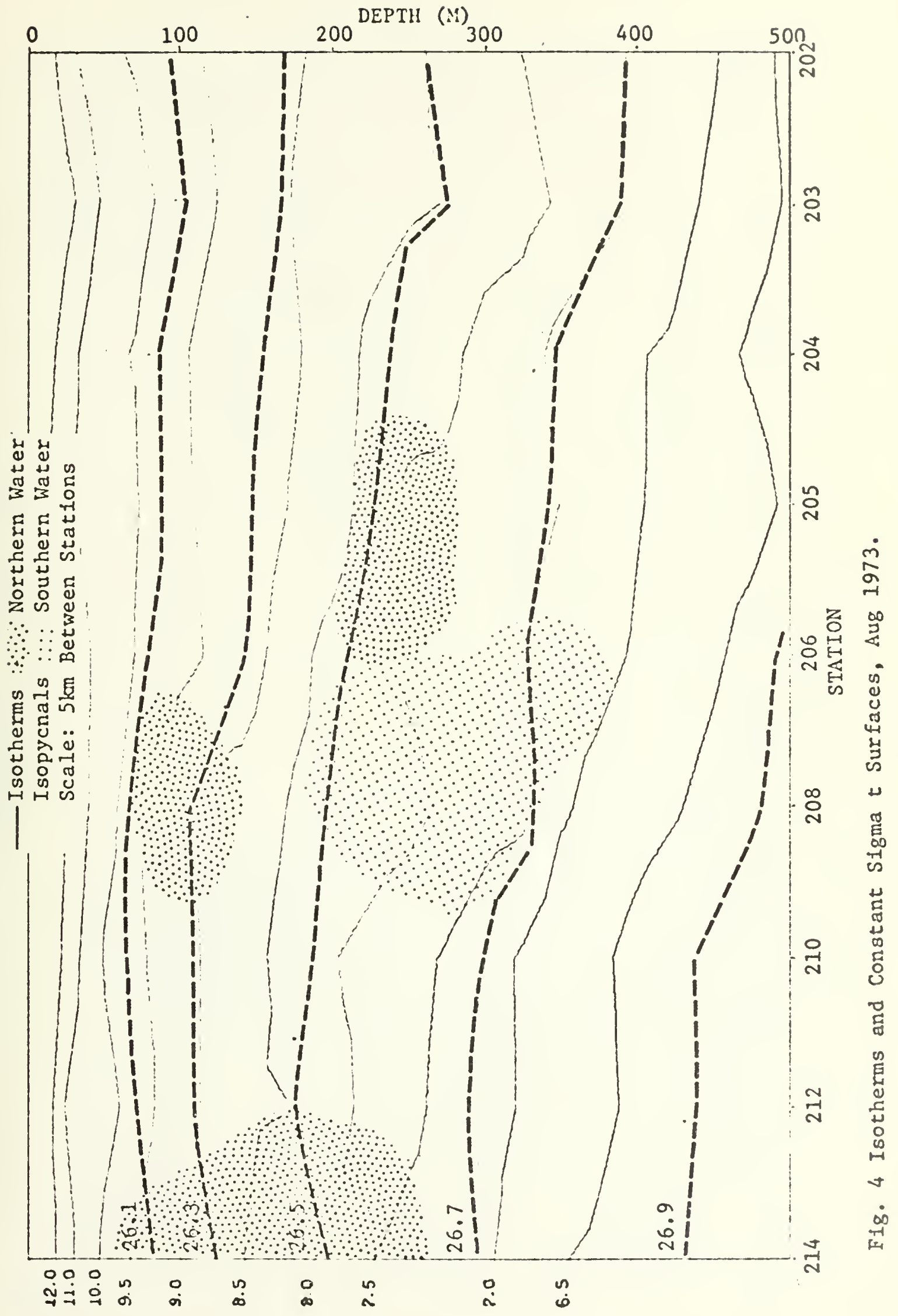





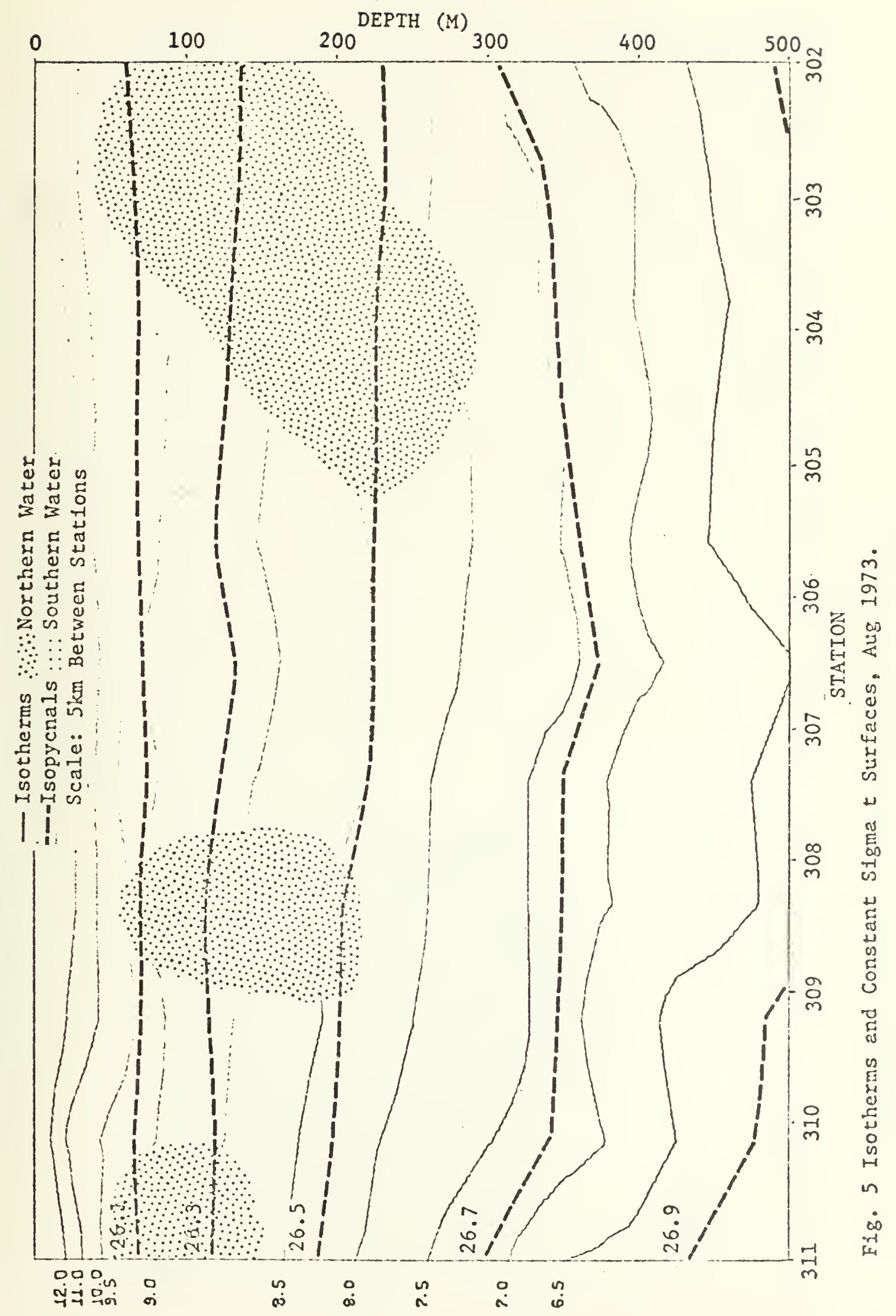





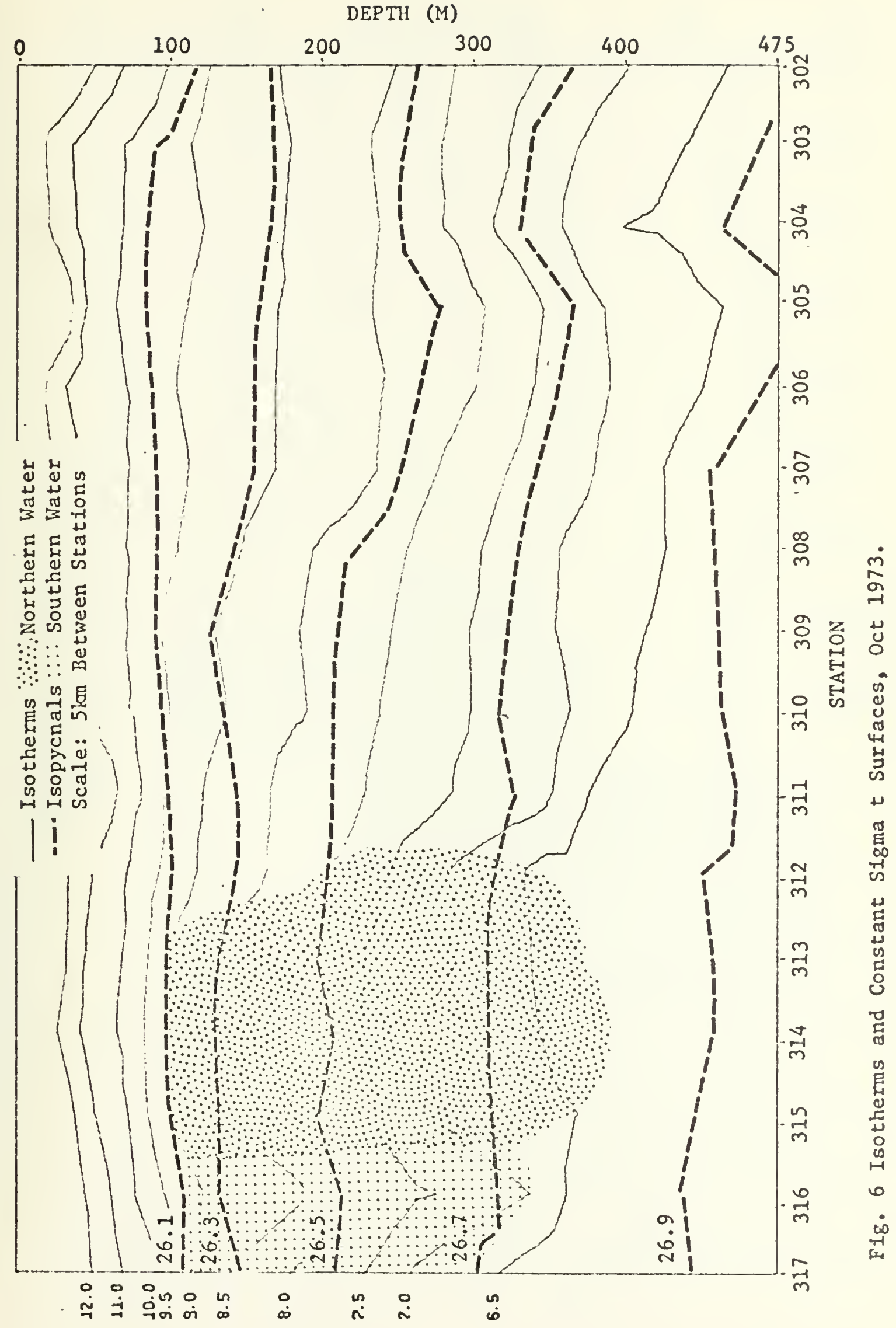





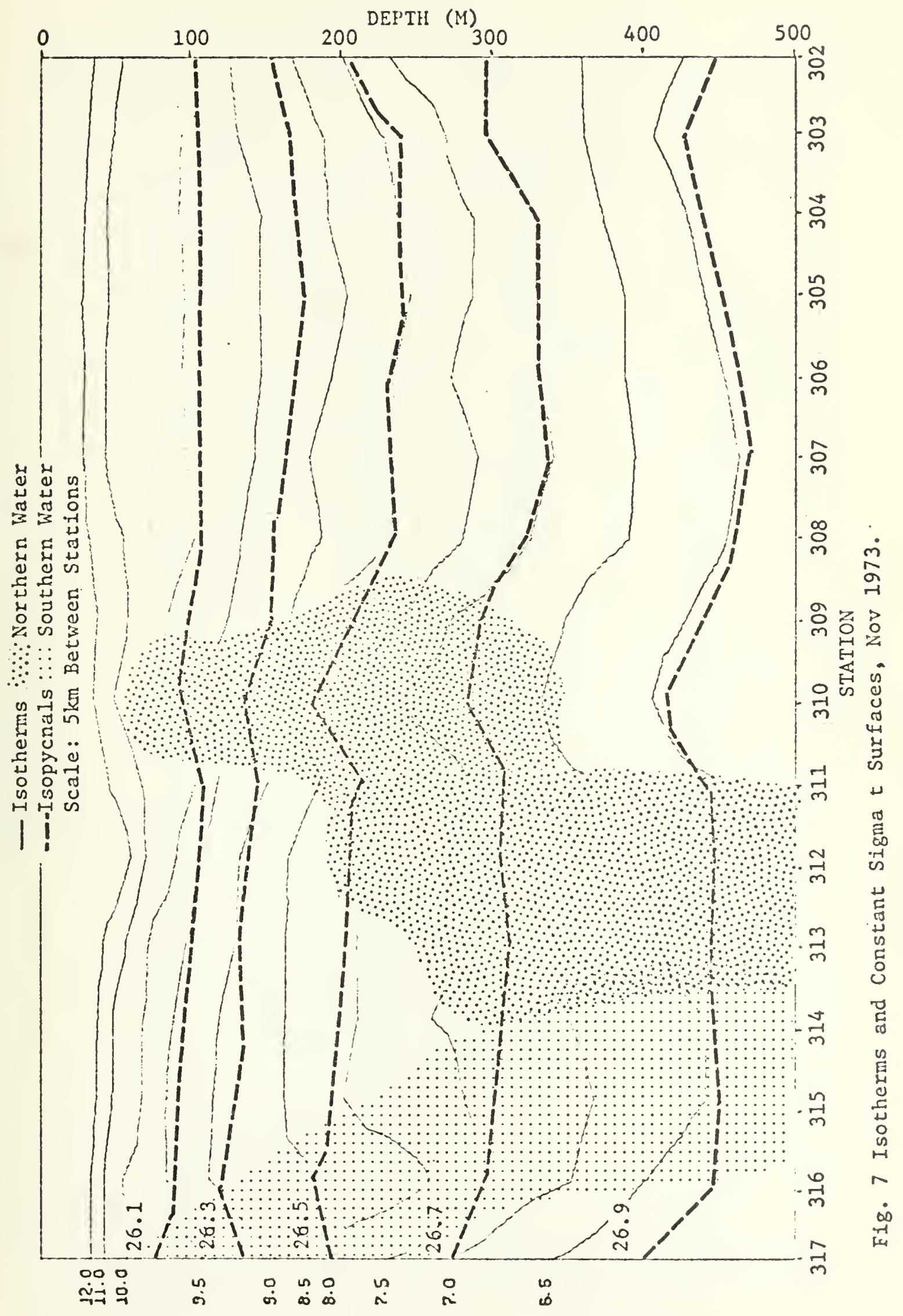





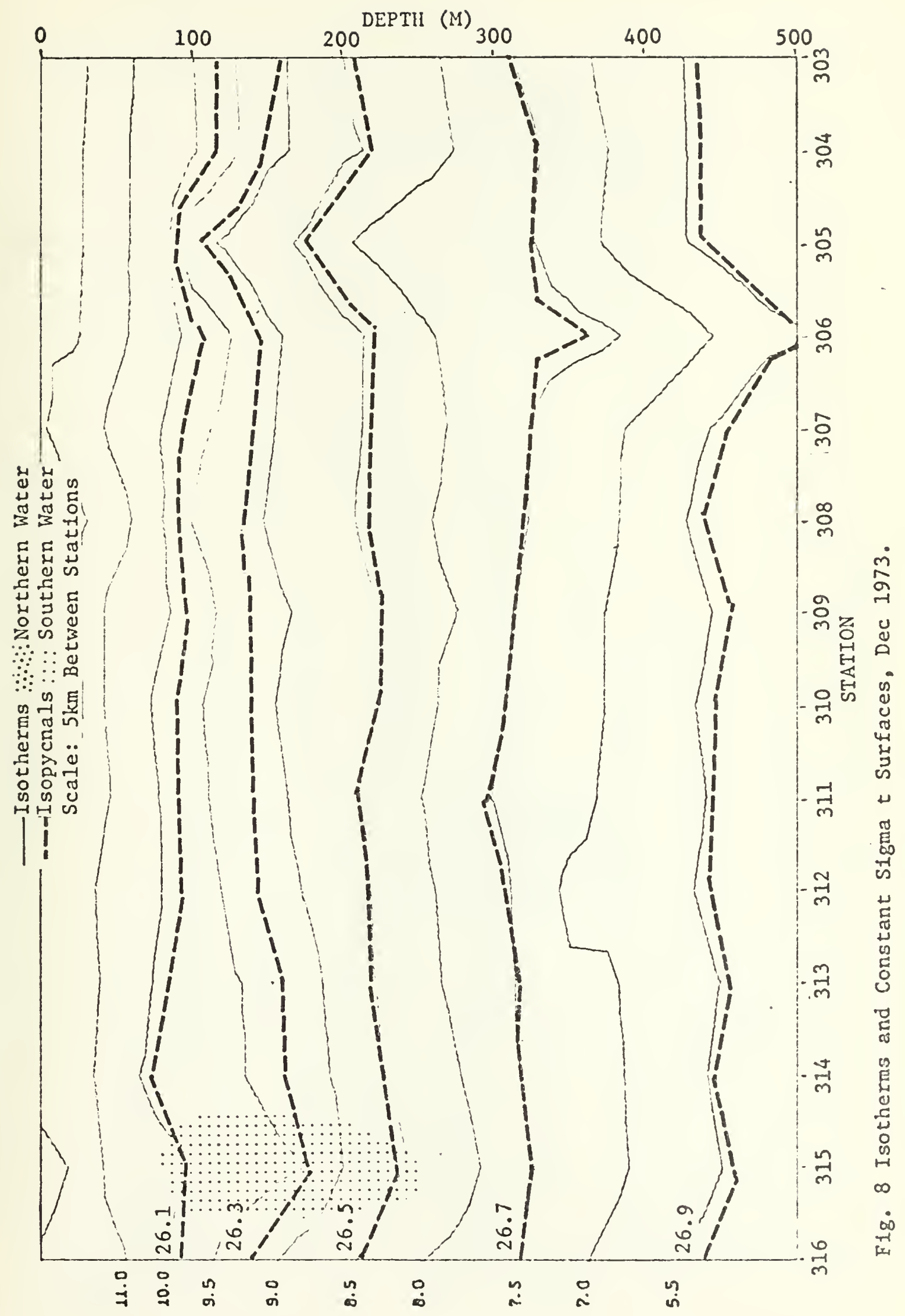





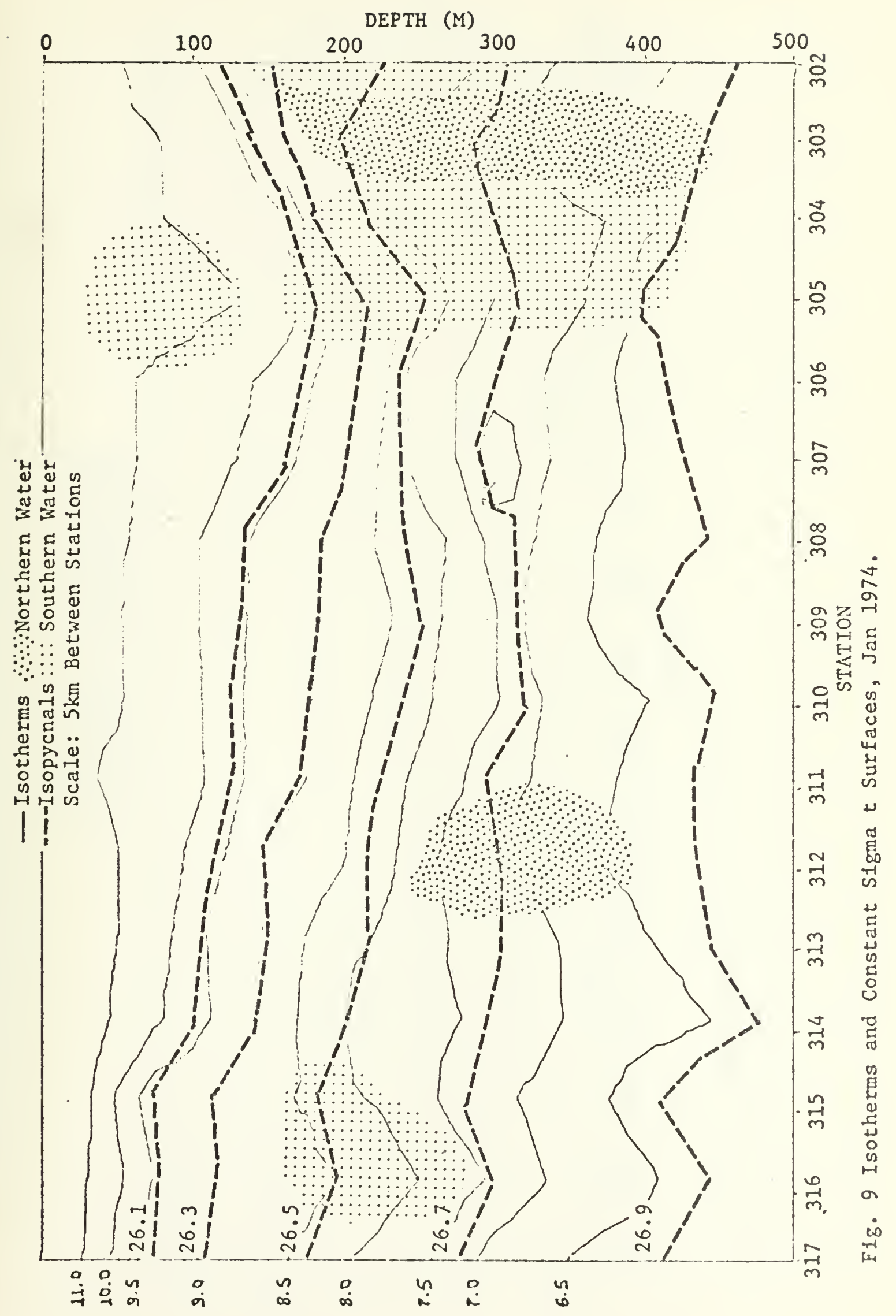





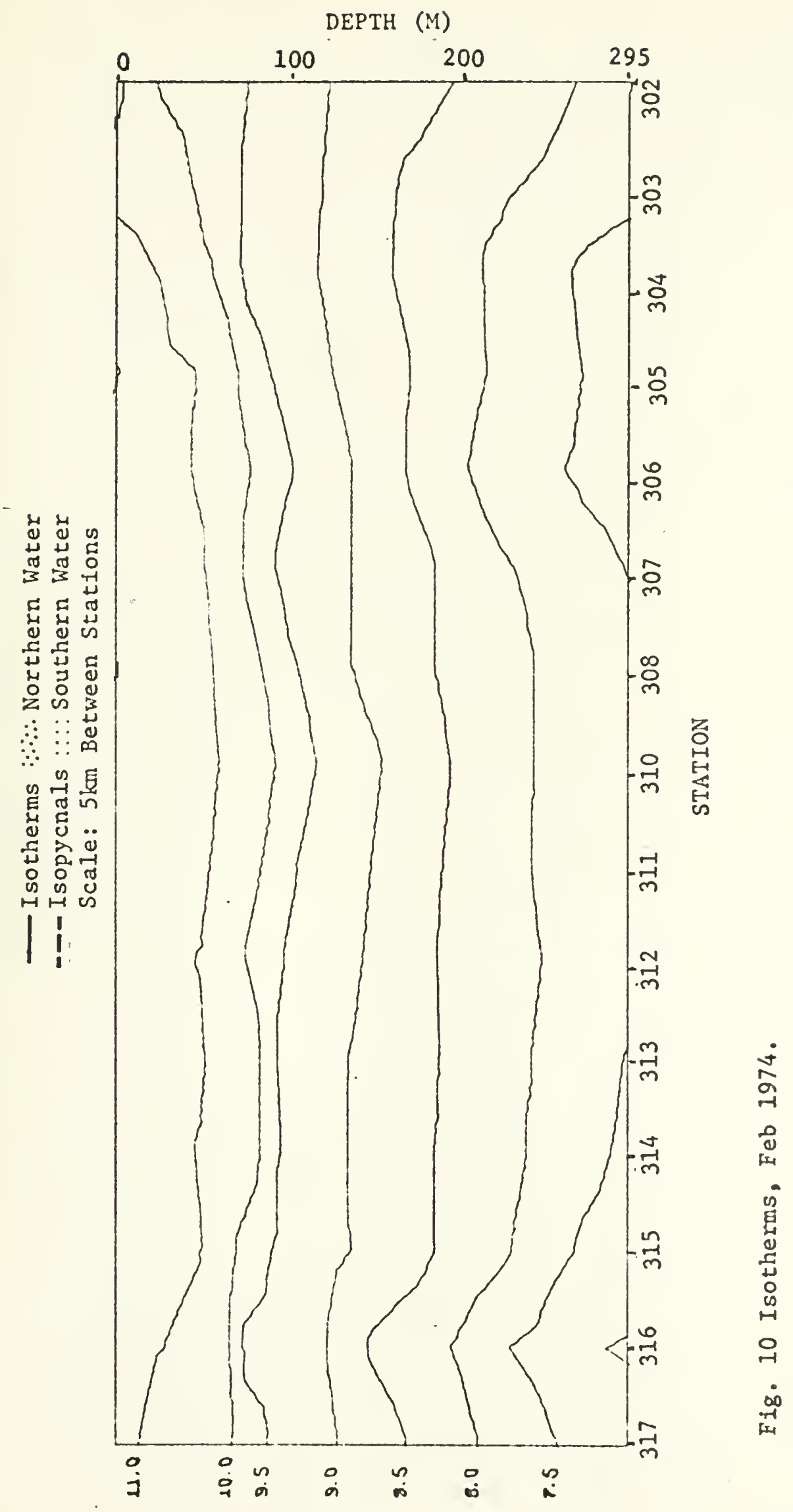





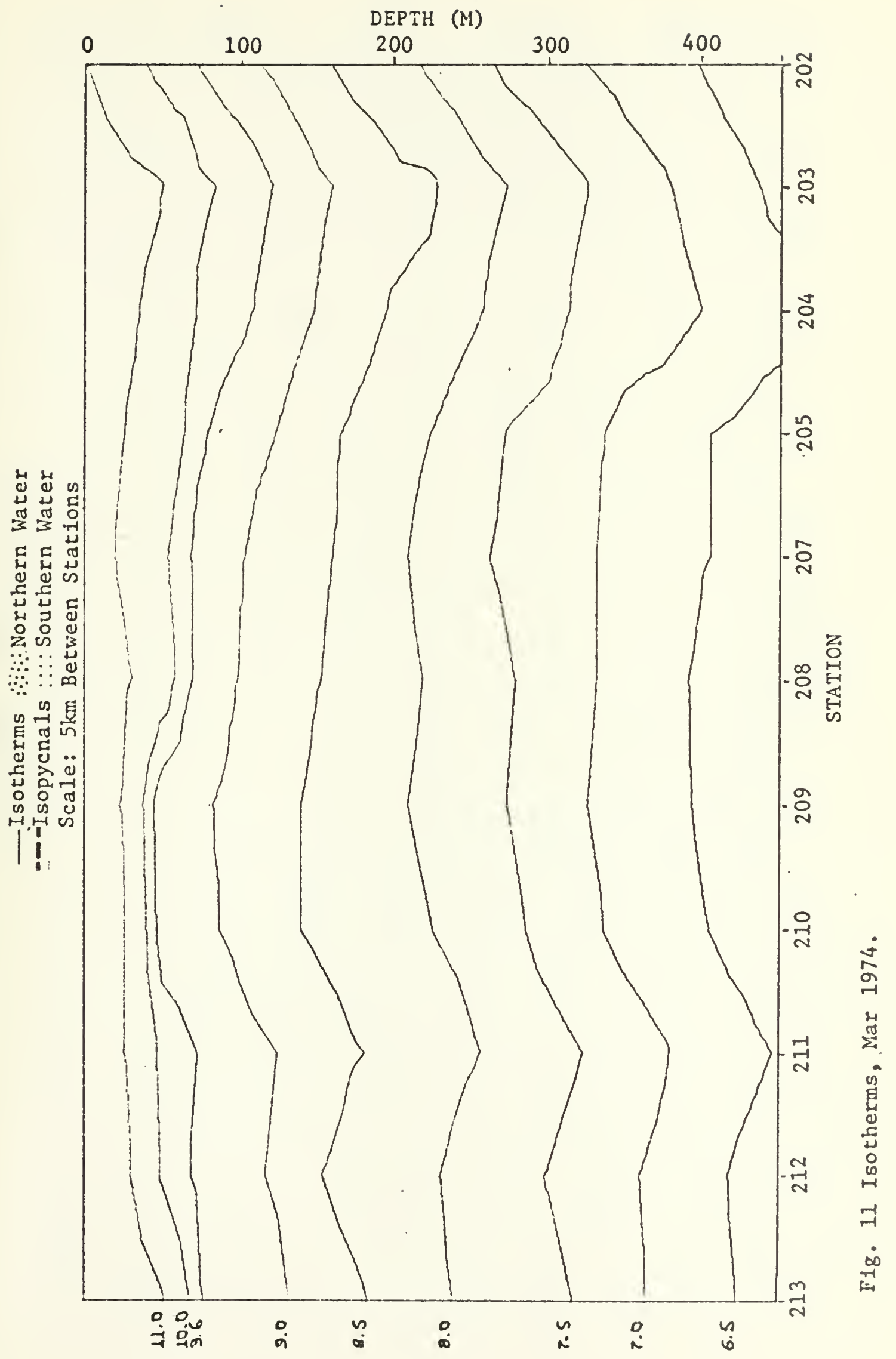





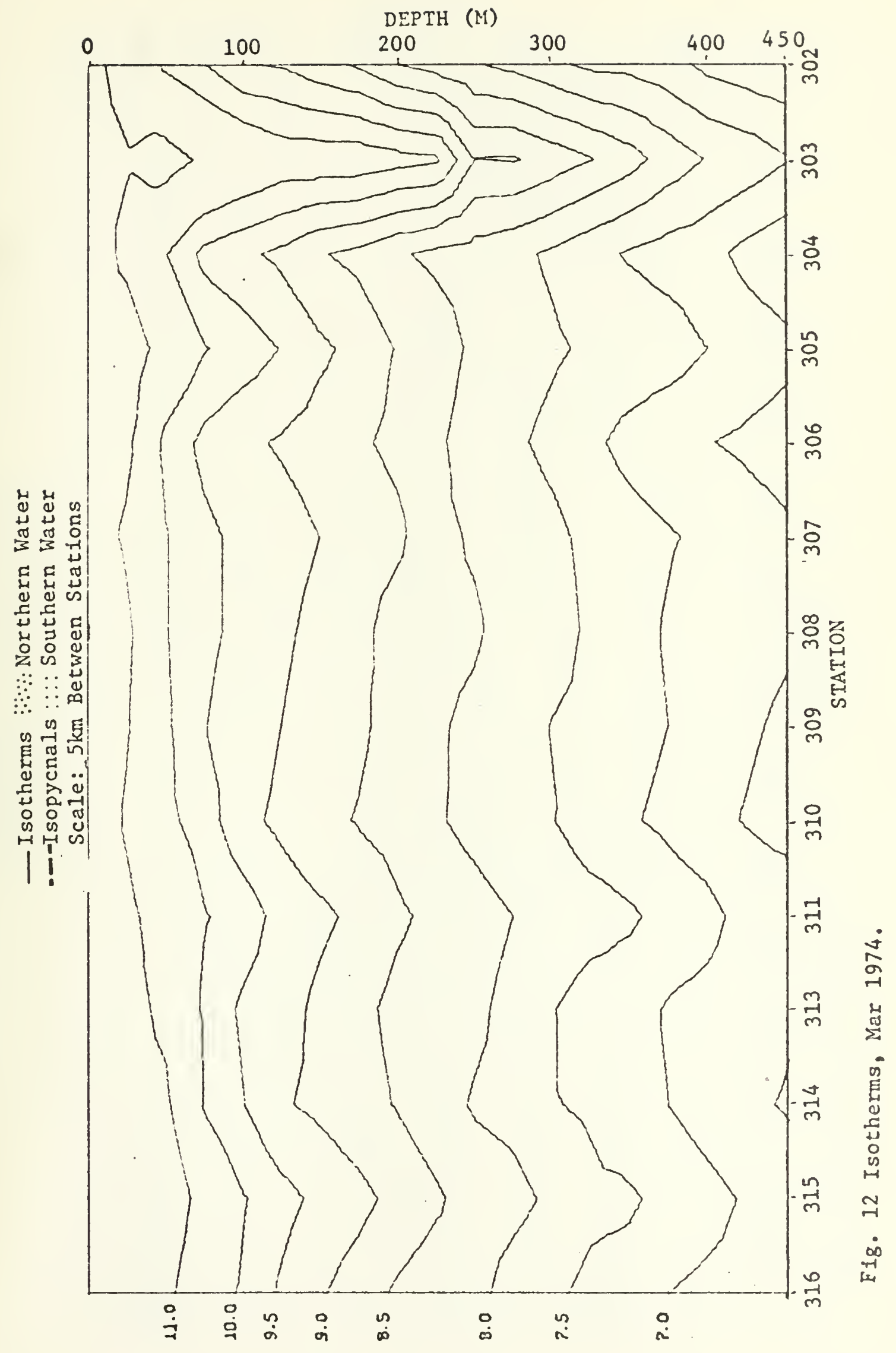





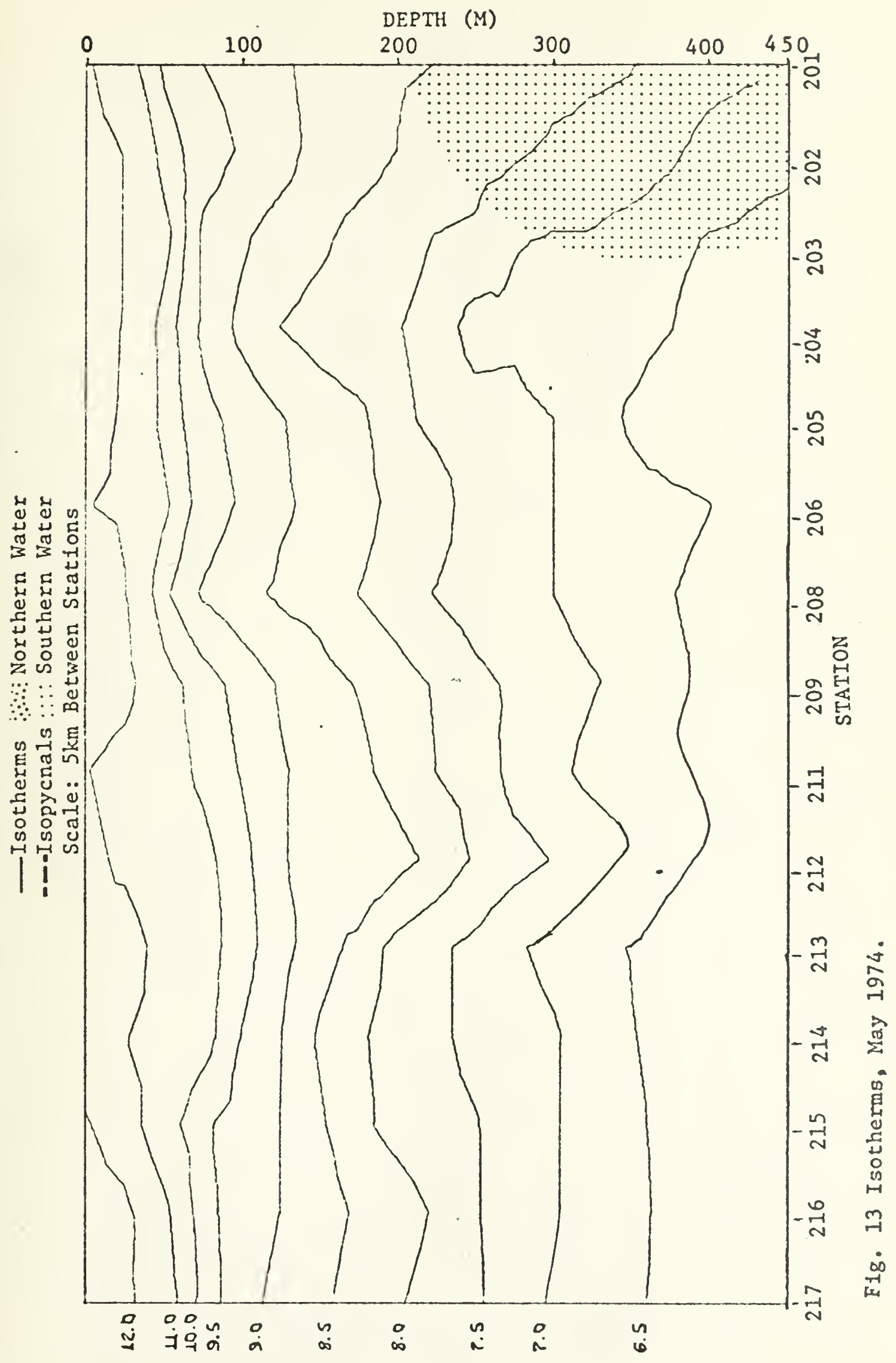





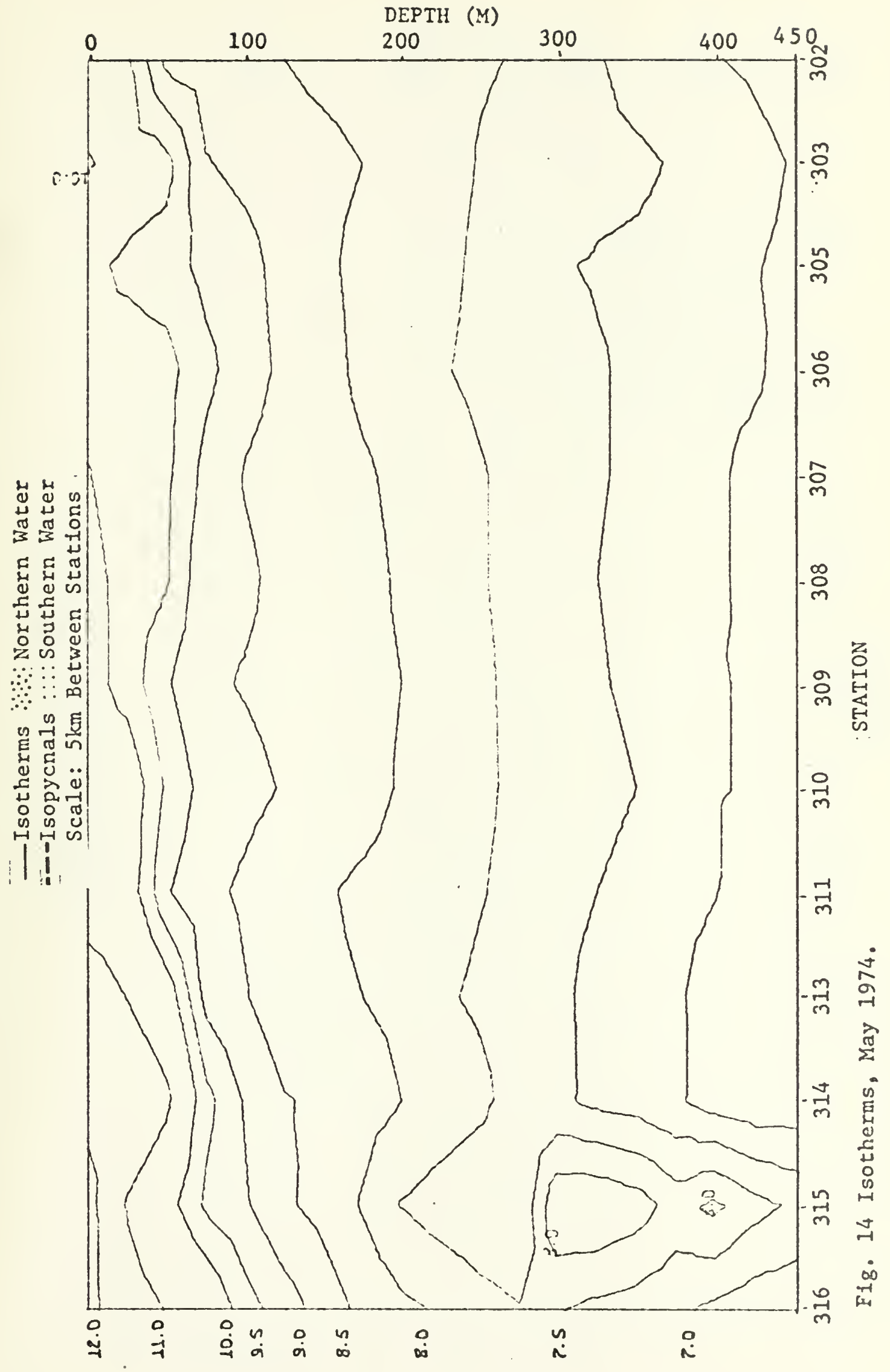





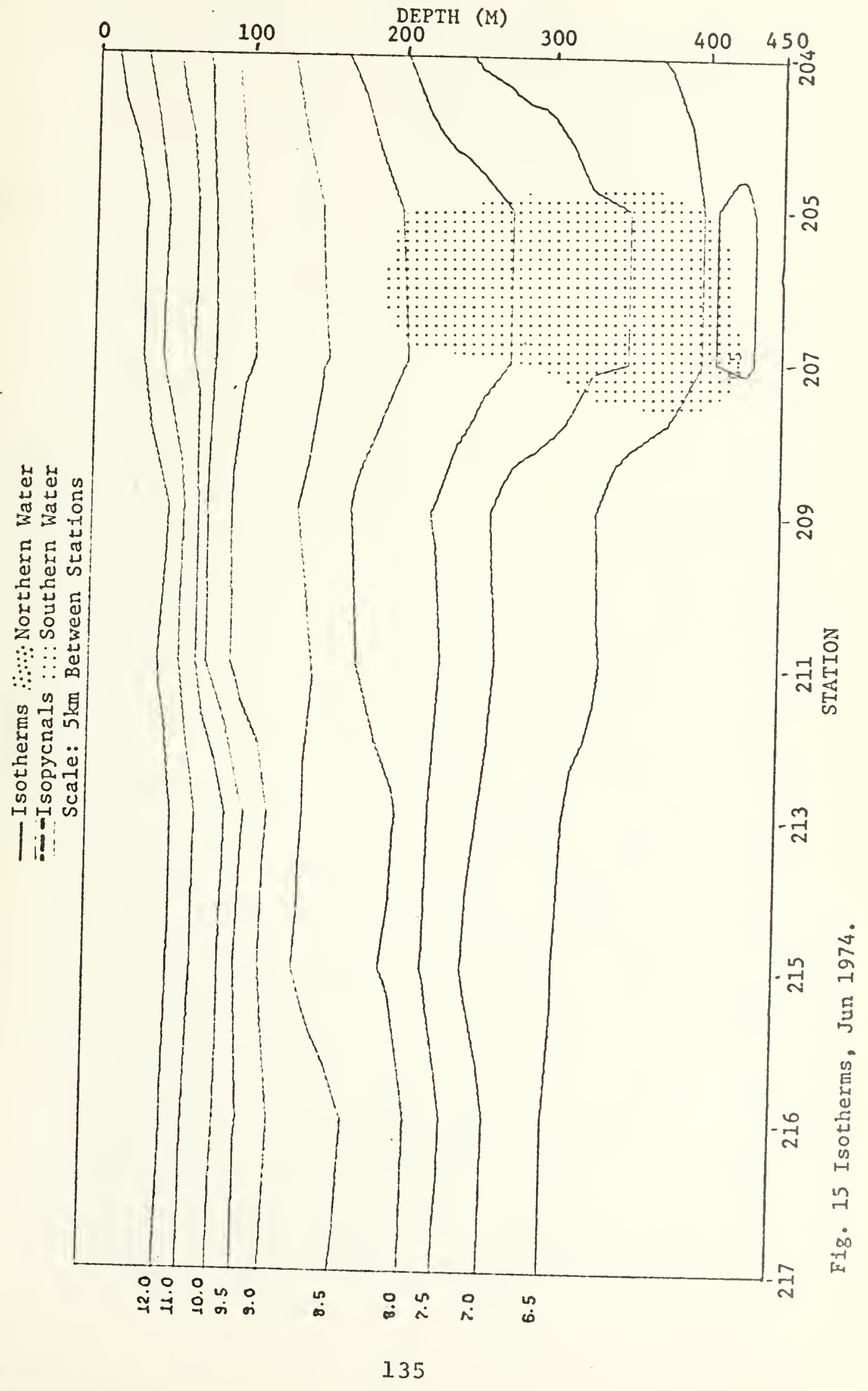





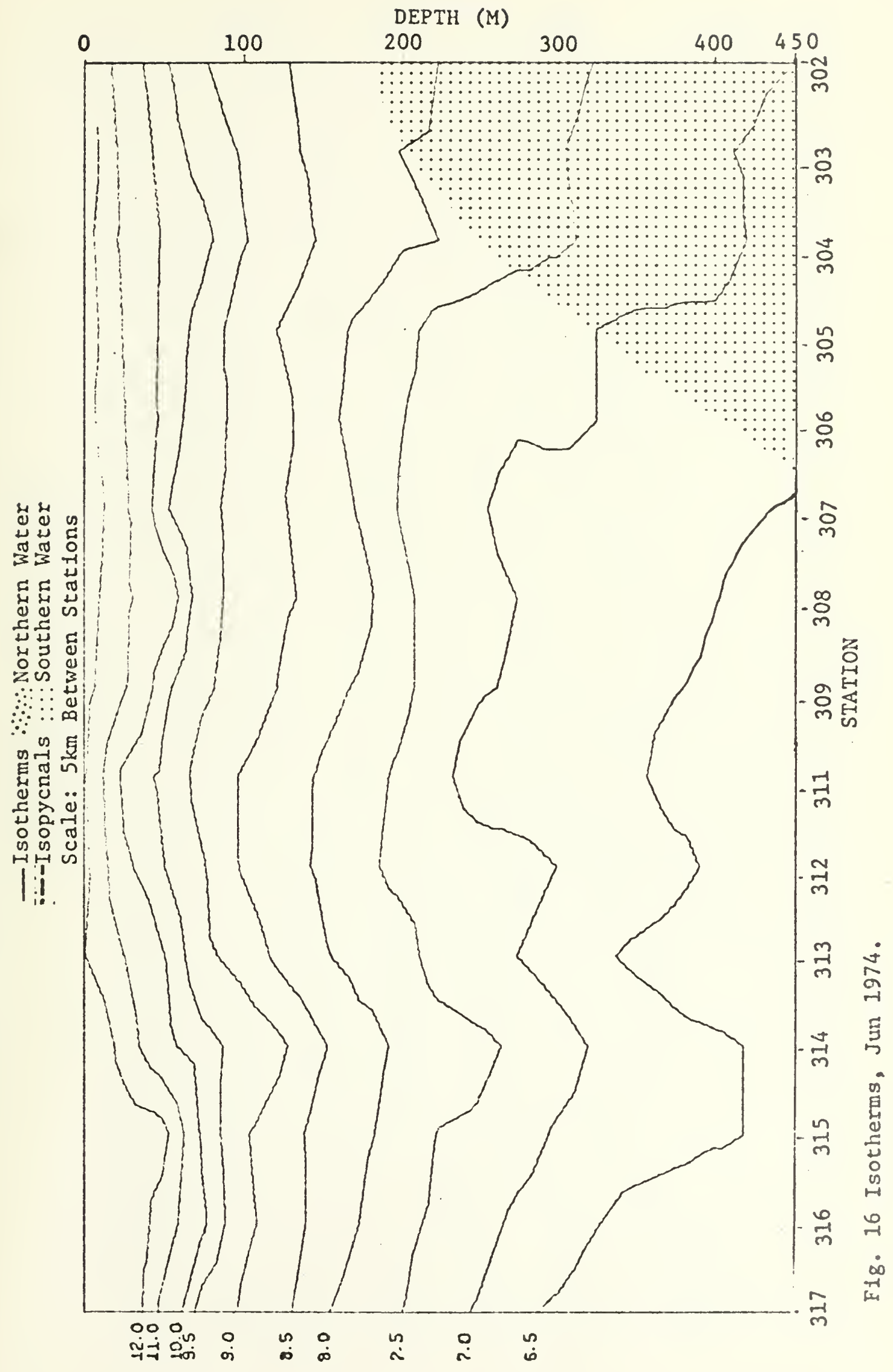





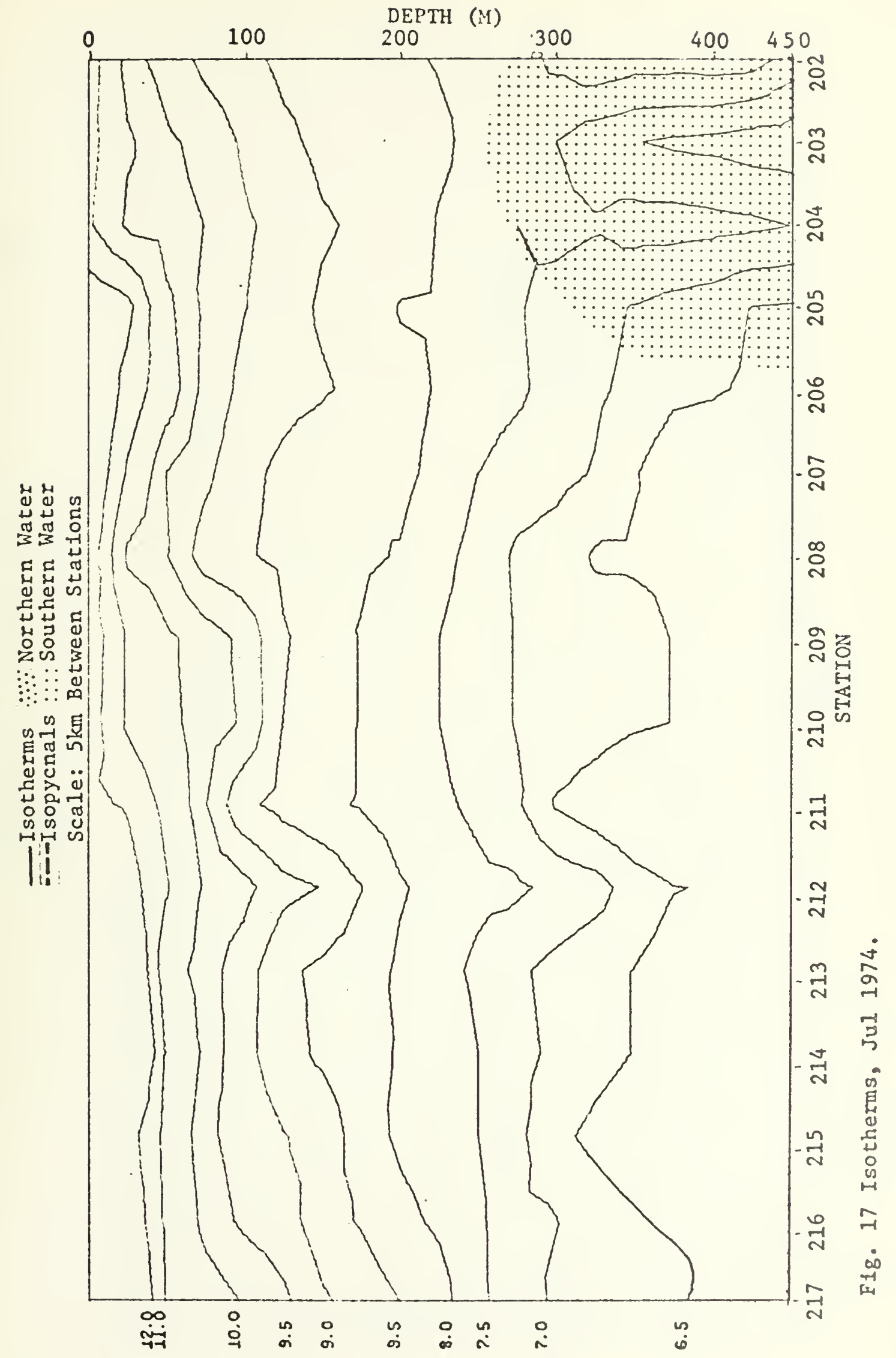





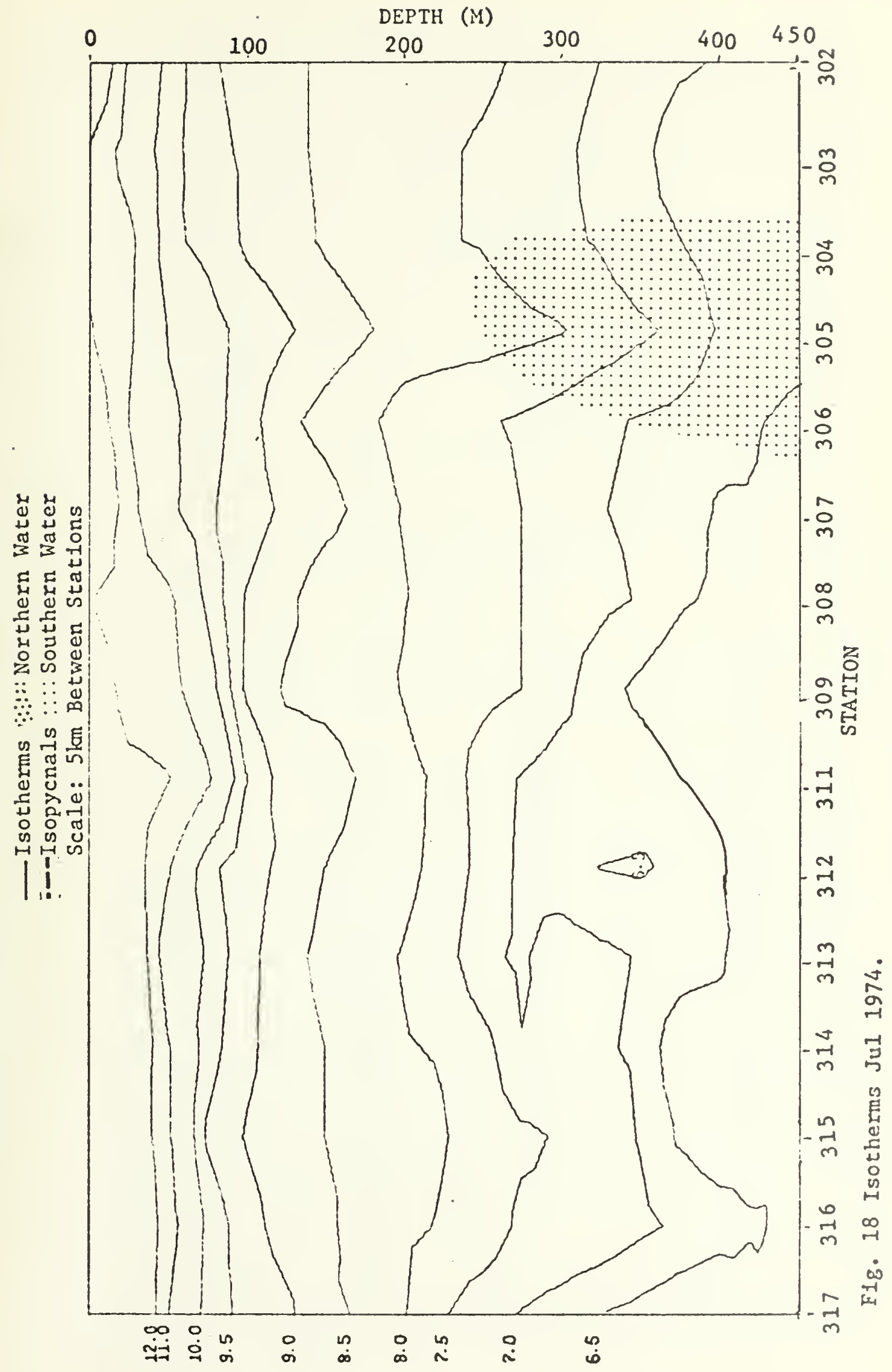





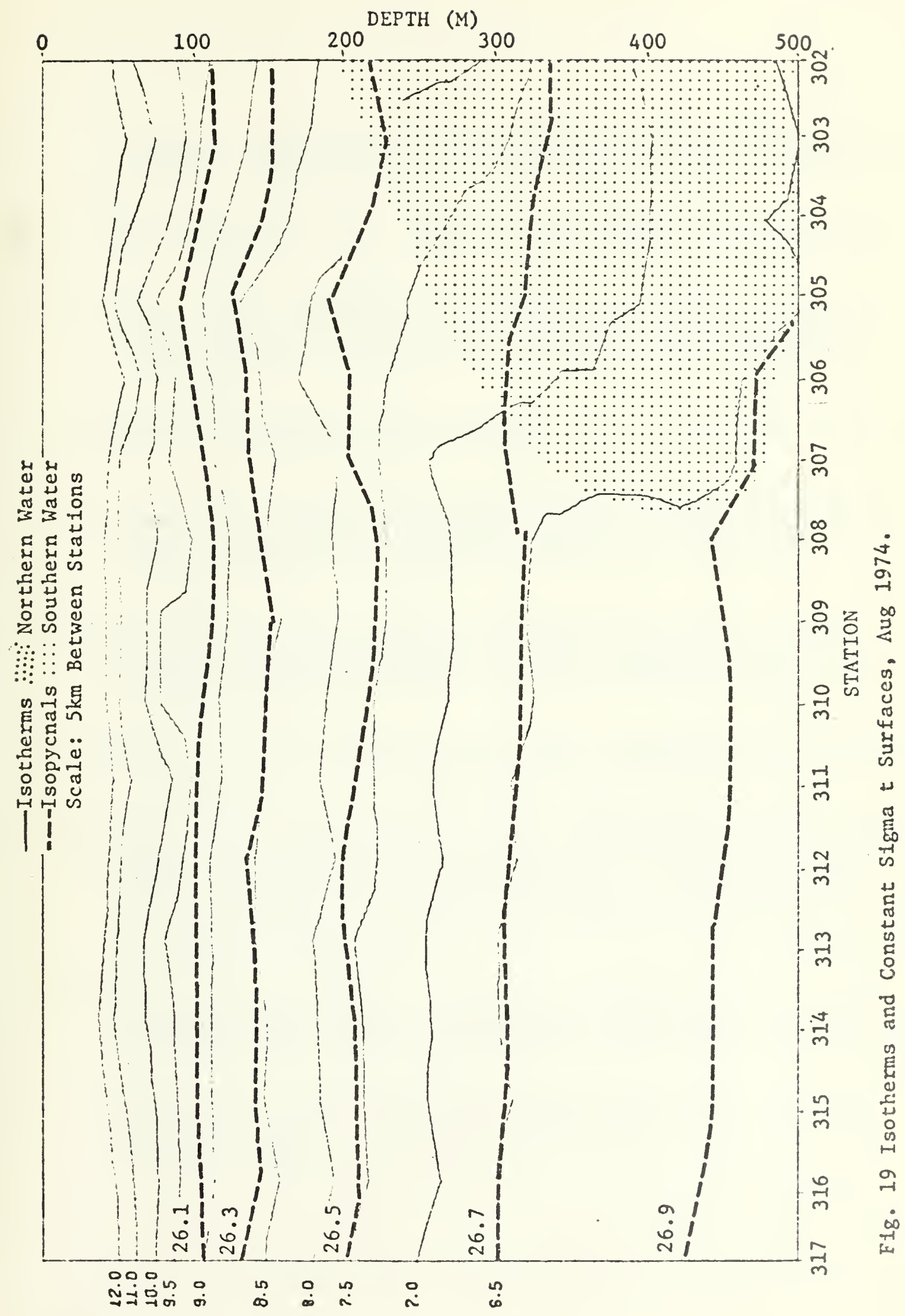





\section{BIBLIOGRAPHY}

1. Brown, R.L., Geostrophic Circulation off the Coast of Central California, Master's Thesis, Naval Postgraduate School, Monterey, California, March 1974.

2. Calma Bulletin 303 B, Calma Company Model 303 Digitizer, Calma Company, Santa Clara, California, 1967.

3. Edmisten, J.R., California Nearshore Surface Currents: Past Observations and Recent Remote Sensing Information, CE 299, University of California, Berkeley, California, June 1974.

4. Glossary of Oceanographic Terms, 2nd. ed., SP-35, pp. 28, 45, Naval Oceanographic Office, 1966.

5. Molnar, D.L., California Undercurrent Reconnaissance between Monterey and Santa Barbara, Master's Thesis, Naval Postgraduate School, Monterey, California, September 1972 .

6. Naval Postgraduate School Technical Note No. 021l-08, Procedures for Converting 7-Track Magnetic Tapes to 9-Track Magnetic Tapes, by S. D. Raney, 3rd. ed., February 1973 .

7. Neumann, G. and Pierson, W.J., Jr., Principles of Physical Oceanography, Prentice-Hall, Inc., 1966.

8. Pirie, D. M., and Steller, D. D., California Coast Nearshore Processes Study Final Report ERTS-1 Experiment $\# 088$, Contract No. S-70257-Ag, U. S. Army Engineer District, San Francisco, California, and Geoscience Division, Geoscience International, Inc., Seal Beach, California, May 1972.

9. Sverdrup, H.U., Johnson, M.W., and Fleming, R.H., The Oceans: Their Physics, Chemistry, and General Biology, Prentice-Hall, Inc., 1942.

10. Wickham, J.B., "Observations of the California Countercurrent," Journal of Marine Research, Vol. 33 (in press), $19 \overline{75 .}$ 

No. Copies

1. Department of Oceanography, Code 58

Naval Postgraduate School

Monterey, California 93940

2. Oceanographer of the Navy

Hoffman Building No. 2

200 Stovall Street

Alexandria, Virginia 22332

3. Office of Naval Research

Code 480

Arlington, Virginia 22217

4. Dr. Robert E. Stevenson

1

Scientific Liaison Office, ONR

Scripps Institution of Oceanography

La Jolla, California 92037

5. Library, Code 3330

Naval Oceanographic Office

Washington, D. C. 20373

6. SIO Library

University of California, San Diego

P. O. Box 2367

La Jolla, California 92037

7. Department of Oceanography Library

University of Washington

Seattle, Washington 98105

8. Department of Oceanography Library

Oregon State University

Corvallis, Oregon 97331

9. Commanding Officer

Fleet Numerical Weather Central

Monterey, California 93940

10. Commanding Officer

Environmental Prediction Research Facility

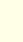



12. Defense Documentation Center Cameron Station

Alexandria, Virginia 22314

13. Library (Code 0212)

Naval Postgraduate School

Monterey, California 93940

14. Professor J. B. Wickham Code $58 \mathrm{Wk}$

Naval Postgraduate School

Monterey, California 93940

15. LT Richard E. Greer

Navigation

USS JOHN F. KENNEDY (CV67)

FPO New York 09501

16. LT John G. Hughes

U. S. National Support Unit NAVSOUTH

FPO New York 09529

17. LT Richard E. Blumberg

DMAHC CUBI PT RP

Box 59

FPO San Francisco 96654 

Thes is

B583

C.1
$4 \div \div 3$

B l umberg

Mesoscale spatial and temporal variations 5 of water mass characteristics in the California current region off Monterey Bay in 1973-1974.

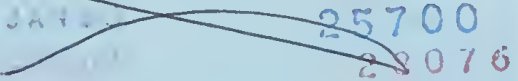

6 int $87 \quad 510334$ Thes is B583

C.1 Blumberg Mesoscale spatial and temporal variations of water mass characteristics in the California current region off Monterey Bay in 1973-1974. 


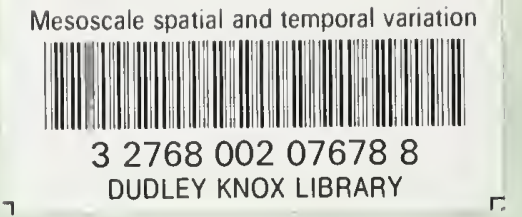

